\title{
Lectures on Arithmetic Noncommutative Geometry
}

\author{
Matilde Marcolli
}



And indeed there will be time

To wonder "Do I dare?" and, "Do I dare?"

Time to turn back and descend the stair.

Do I dare

Disturb the Universe?

For I have known them all already, known them all;

Have known the evenings, mornings, afternoons,

I have measured out my life with coffee spoons.

I should have been a pair of ragged claws

Scuttling across the floors of silent seas.

No! I am not Prince Hamlet, nor was meant to be;

Am an attendant lord, one that will do

To swell a progress, start a scene or two

At times, indeed, almost ridiculous-

Almost, at times, the Fool.

We have lingered in the chambers of the sea

By sea-girls wreathed with seaweed red and brown

Till human voices wake us, and we drown.

(T.S. Eliot, "The Love Song of J. Alfred Prufrock") 



\section{Contents}

Preface ix

Chapter 1. Ouverture 1

1. The NCG dictionary 3

2. Noncommutative spaces 4

3. Spectral triples 6

4. Why noncommutative geometry? 12

$\begin{array}{lll}\text { Chapter 2. Noncommutative modular curves } & 15\end{array}$

1. Modular curves $\quad 15$

2. The noncommutative boundary of modular curves 22

3. Limiting modular symbols $\quad 27$

4. Hecke eigenforms $\quad 39$

5. Selberg zeta function 41

6. The modular complex and $K$-theory of $\mathrm{C}^{*}$-algebras 42

7. Intermezzo: Chaotic Cosmology 44

Chapter 3. Quantum statistical mechanics and Galois theory 51

1. Quantum Statistical Mechanics 53

2. The Bost-Connes system 56

3. Noncommutative Geometry and Hilbert's 12th problem 61

4. The $\mathrm{GL}_{2}$ system 64

5. Quadratic fields 70

Chapter 4. Noncommutative geometry at arithmetic infinity 81

$\begin{array}{ll}\text { 1. Schottky uniformization } & 81\end{array}$

2. Dynamics and noncommutative geometry 88

3. Arithmetic infinity: archimedean primes 93

4. Arakelov geometry and hyperbolic geometry 97

5. Intermezzo: Quantum gravity and black holes 100

6. Dual graph and noncommutative geometry 105

7. Arithmetic varieties and $L$-factors 109

8. Archimedean cohomology 115

$\begin{array}{ll}\text { Chapter 5. Vistas } & 125\end{array}$

$\begin{array}{ll}\text { Bibliography } & 131\end{array}$ 



\section{Preface}

Noncommutative geometry nowadays looks as a vast building site.

On the one hand, practitioners of noncommutative geometry (or geometries) already built up a large and swiftly growing body of exciting mathematics, challenging traditional boundaries and subdivisions.

On the other hand, noncommutative geometry lacks common foundations: for many interesting constructions of "noncommutative spaces" we cannot even say for sure which of them lead to isomorphic spaces, because they are not objects of an all-embracing category (like that of locally ringed topological spaces in commutative geometry).

Matilde Marcolli's lectures reflect this spirit of creative growth and interdisciplinary research.

She starts Chapter 1 with a sketch of philosophy of noncommutative geometry à la Alain Connes. Briefly, Connes suggests imagining $C^{*}$-algebras as coordinate rings. He then supplies several bridges to commutative geometry by his construction of "bad quotients" of commutative spaces via crossed products and his treatment of noncommutative Riemannian geometry. Finally, algebraic tools like $K$-theory and cyclic cohomology serve to further enhance geometric intuition.

Marcolli then proceeds to explaining some recent developments drawing upon her recent work with several collaborators. A common thread in all of them is the study of various aspects of uniformization: classical modular group, Schottky groups. The modular group acts upon the complex half plane, partially compactified by cusps: rational points of the boundary projective line. The action becomes "bad" at irrational points, and here is where noncommutative geometry enters the game. A wealth of classical number theory is encoded in the co-

efficients of modular forms, their Mellin transforms, Hecke operators and modular symbols. Their counterparts living at the noncommutative boundary have only recently started to unravel themselves, and Marcolli gives a beautiful overview of what is already understood in Chapters 2 and 3. 
Schottky uniformization provides a visualization of Arakelov's geometry at arithmetic infinity, which serves as the main motivation of Chapter 4.

Among the most tantalizing developments is the recurrent emergence of patches of common ground for number theory and theoretical physics.

In fact, one can present in this light the famous theorem of young Gauss characterising regular polygons that can be constructed using only ruler and compass. In his Tagebuch entry of March 30 he announced that a regular 17-gon has this property.

Somewhat modernizing his discovery, one can present it in the following way.

In the complex plane, roots of unity of degree $n$ form vertices of a regular $n$-gone. Hence it makes sense to imagine that we study the ruler and compass constructions as well not in the Euclidean, but in the complex plane. This has an unexpected consequence: we can characterize the set of all points constructible in this way as the maximal Galois 2-extension of $\mathbb{Q}$. It remains to calculate the Galois group of $\mathbb{Q}\left(e^{2 \pi i / 17}\right)$ : since it is cyclic of order 16 , this root of unity is constructible. Moreover, the same is true for all $p$-gons where $p$ is a prime of the form $2^{n}+1$ but not for other primes.

A remarkable feature of this result is the appearance of a hidden symmetry group. In fact, the definitions of a regular $n$-gon and ruler and compass constructions are initially formulated in terms of Euclidean plane geometry and suggest that the relevant symmetry group must be that of rigid rotations $S O(2)$, eventually extended by reflections and shifts. This conclusion turns out to be totally misleading: instead, one should rely upon $\operatorname{Gal}(\overline{\mathbb{Q}} / \mathbb{Q})$. The action of the latter group upon roots of unity of degree $n$ factors through the maximal abelian quotient and is given by $\zeta \mapsto \zeta^{k}$, with $k$ running over all $k \bmod n$ with $(k, n)=1$, whereas the action of the rotation group is given by $\zeta \mapsto \zeta_{0} \zeta$ with $\zeta_{0}$ running over all $n$-th roots. Thus, the $\operatorname{Gal}(\overline{\mathbb{Q}} / \mathbb{Q})-$ symmetry does not conserve angles between vertices which seem to be basic for the initial problem. Instead, it is compatible with addition and multiplication of complex numbers, and this property proves to be crucial.

With some stretch of imagination, one can recognize in the Euclidean avatar of this picture a physics flavor (putting it somewhat pompously, it appeals to the kinematics of 2 -dimensional rigid bodies 
in gravitational vacuum), whereas the Galois avatar definitely belongs to number theory.

In the Marcolli lectures, stressing number theory, physics themes pop up at the end of Chapter 2 (Chaotic Cosmology in general relativity), the beginning of Chapter 3 (formalism of quantum statistical mechanics), and finally, sec. 5 of Chapter 4 where some models of black holes in general relativity turn out to have the same mathematical description as $\infty$-adic fibers of curves in Arakelov geometry. The reemergence of Gauss' Galois group Gal ${ }^{a b}(\overline{\mathbb{Q}} / \mathbb{Q})$ in Bost-Connes symmetry breaking, and of Gauss' statistics of continued fractions in the Chaotic Cosmology models, shows that connections with classical mathematics are as strong as ever.

Hopefully, this lively exposition will attract young researchers and incite them to engage themselves in exploration of the rich new territory.

Yuri I. Manin. Bonn, March 17, 2005. 


\section{CHAPTER 1}

\section{Ouverture}

Noncommutative geometry, as developed by Connes starting in the early ' 80 s $([\mathbf{1 6}],[\mathbf{1 8}],[\mathbf{2 1}])$, extends the tools of ordinary geometry to treat spaces that are quotients, for which the usual "ring of functions", defined as functions invariant with respect to the equivalence relation, is too small to capture the information on the "inner structure" of points in the quotient space. Typically, for such spaces functions on the quotients are just constants, while a nontrivial ring of functions, which remembers the structure of the equivalence relation, can be defined using a noncommutative algebra of coordinates, analogous to the noncommuting variables of quantum mechanics. These "quantum spaces" are defined by extending the Gel'fand-Naimark correspondence

$$
X \text { loc.comp. Hausdorff space } \Leftrightarrow C_{0}(X) \text { abelian } C^{*} \text {-algebra }
$$

by dropping the commutativity hypothesis in the right hand side. The correspondence then becomes a definition of what is on the left hand side: a noncommutative space.

Such quotients are abundant in nature. They arise, for instance, from foliations. Several recent results also show that noncommutative spaces arise naturally in number theory and arithmetic geometry. The first instance of such connections between noncommutative geometry and number theory emerged in the work of Bost and Connes [9], which exhibits a very interesting noncommutative space with remarkable arithmetic properties related to class field theory. This reveals a very useful dictionary that relates the phenomena of spontaneous symmetry breaking in quantum statistical mechanics to the mathematics of Galois theory. This space can be viewed as the space of 1-dimensional $\mathbb{Q}$-lattices up to scale, modulo the equivalence relation of commensurability ( $c f .[32])$. This space is closely related to the noncommutative space used by Connes to obtain a spectral realization of the zeros of the Riemann zeta function, [23]. In fact, this is again the space of commensurability classes of 1 -dimensional $\mathbb{Q}$-lattices, but with the scale factor also taken into account.

More recently, other results that point to deep connections between noncommutative geometry and number theory appeared in the work 
of Connes and Moscovici [41] [42] on the modular Hecke algebras. This shows that the Rankin-Cohen brackets, an important algebraic structure on modular forms [110], have a natural interpretation in the language of noncommutative geometry, in terms of the Hopf algebra of the transverse geometry of codimension one foliations. The modular Hecke algebras, which naturally combine products and action of Hecke operators on modular forms, can be viewed as the "holomorphic part" of the algebra of coordinates on the space of commensurability classes of 2-dimensional $\mathbb{Q}$-lattices constructed in joint work of Connes and the author [32].

Cases of occurrences of interesting number theory within noncommutative geometry can be found in the classification of noncommutative three-spheres by Connes and Dubois-Violette [28] [29]. Here the corresponding moduli space has a ramified cover by a noncommutative nilmanifold, where the noncommutative analog of the Jacobian of this covering map is expressed naturally in terms of the ninth power of the Dedekind eta function. Another such case occurs in Connes' calculation [25] of the explicit cyclic cohomology Chern character of a spectral triple on $S U_{q}(2)$ defined by Chakraborty and Pal [13].

Other instances of noncommutative spaces that arise in the context of number theory and arithmetic geometry can be found in the noncommutative compactification of modular curves of [26], [83]. This noncommutative space is again related to the noncommutative geometry of $\mathbb{Q}$-lattices. In fact, it can be seen as a stratum in the compactification of the space of commensurability classes of 2-dimensional $\mathbb{Q}$-lattices $(c f .[32])$.

Another context in which noncommutative geometry provides a useful tool for arithmetic geometry is in the description of the totally degenerate fibers at "arithmetic infinity" of arithmetic varieties over number fields, analyzed in joint work of the author with Katia Consani $([44],[45],[46],[47])$.

The present text is based on a series of lectures given by the author at Vanderbilt University in May 2004, as well as on previous series of lectures given at the Fields Institute in Toronto (2002), at the University of Nottingham (2003), and at CIRM in Luminy (2004).

The main focus of the lectures is the noncommutative geometry of modular curves (following [83]) and of the archimedean fibers of arithmetic varieties (following [44]). A chapter on the noncommutative space of commensurability classes of 2-dimensional $\mathbb{Q}$-lattices is also included (following [32]). The text reflects very closely the style of the lectures. In particular, we have tried more to convey the general picture than the details of the proofs of the specific results. Though 
many proofs have not been included in the text, the reader will find references to the relevant literature, where complete proofs are provided (in particular [32], [44], [38], and [83]).

More explicitly, the text is organized as follows:

- We start by recalling a few preliminary notions of noncommutative geometry (following $[\mathbf{2 1}]$ ).

- The second chapter describes how various arithmetic properties of modular curves can be seen by their "noncommutative boundary". This part is based on the joint work of Yuri Manin and the author. The main references are [83], [84], [85].

- The third chapter includes an account of the work of Connes and the author $[\mathbf{3 2}]$ on the noncommutative geometry of commensurability classes of $\mathbb{Q}$-lattices. It also includes a discussion of the relation of the noncommutative space of commensurability classes of $\mathbb{Q}$-lattices to the Hilbert 12 th problem of explicit class field theory and a section on the results of Connes, Ramachandran and the author [38] on the construction of a quantum statistical mechnical system that fully recovers the explicit class field theory of imaginary quadratic fields. We also included a brief discussion of Manin's real multiplication program [75] [76] and the problem of real quadratic fields.

- The noncommutative geometry of the fibers at "arithmetic infinity" of varieties over number fields is the content of the remaining chapter, based on joint work of Consani and the author, for which the references are $[44],[45],[46],[47],[48]$. This chapter also contains a detailed account of Manin's formula for the Green function of Arakelov geometry for arithmetic surfaces, based on [79], and a proposed physical interpretation of this formula, as in $[82]$.

\section{The NCG dictionary}

There is a dictionary $(c f .[\mathbf{2 1}])$ relating concepts of ordinary geometry to the corresponding counterparts in noncommutative geometry. The entries can be arranged according to the finer structures considered on the underlying space, roughly according to the following table.

\begin{tabular}{|c|c|}
\hline measure theory & von Neumann algebras \\
\hline topology & $C^{*}$-algebras \\
\hline smooth structures & smooth subalgebras \\
\hline Riemannian geometry & spectral triples \\
\hline
\end{tabular}


It is important to notice that, usually, the notions of noncommutative geometry are "richer" than the corresponding entries of the dictionary on the commutative side. For instance, as Connes discovered, noncommutative measure spaces (von Neumann algebras) come endowed with a natural time evolution which is trivial in the commutative case. Similarly, at the level of topology one often sees phenomena that are closer to rigid analytic geometry. This is the case, for instance, with the noncommutative tori $T_{\theta}$, which already at the $C^{*}$-algebra level exhibit moduli that behave much like moduli of one-dimensional complex tori (elliptic curves) in the commutative case.

In the context we are going to discuss this richer structure of noncommutative spaces is crucial, as it permits us to use tools like $C^{*}$ algebras (topology) to study the properties of more rigid spaces like algebraic or arithmetic varieties.

\section{Noncommutative spaces}

The way to assign the algebra of coordinates to a quotient space $X=Y / \sim$ can be explained in a short slogan as follows:

- Functions on $Y$ with $f(a)=f(b)$ for $a \sim b$. Poor!

- Functions $f_{a b}$ on the graph of the equivalence relation. Good!

The second description leads to a noncommutative algebra, as the product, determined by the groupoid law of the equivalence relation, has the form of a convolution product (like the product of matrices).

For sufficiently nice quotients, even though the two notions are not the same, they are related by Morita equivalence, which is the suitable notion of "isomorphism" between noncommutative spaces. For more general quotients, however, the two notions truly differ and the second one is the only one that allows one to continue to make sense of geometry on the quotient space.

A very simple example illustrating the above situation is the following ( $c f$. [24]). Consider the topological space $Y=[0,1] \times\{0,1\}$ with the equivalence relation $(x, 0) \sim(x, 1)$ for $x \in(0,1)$. By the first method one only obtains constant functions $\mathbb{C}$, while by the second method one obtains

$$
\left\{f \in C([0,1]) \otimes M_{2}(\mathbb{C}): f(0) \text { and } f(1) \text { diagonal }\right\}
$$

which is an interesting nontrivial algebra.

The idea of preserving the information on the structure of the equivalence relation in the description of quotient spaces has analogs in Grothendieck's theory of stacks in algebraic geometry. 
2.1. Morita equivalence. In noncommutative geometry, isomorphisms of $C^{*}$-algebras are too restrictive to provide a good notion of isomorphisms of noncommutative spaces. The correct notion is provided by Morita equivalence of $C^{*}$-algebras.

We have equivalent $\mathrm{C}^{*}$-algebras $\mathcal{A}_{1} \sim \mathcal{A}_{2}$ if there exists a bimodule $\mathcal{M}$, which is a right Hilbert $\mathcal{A}_{1}$ module with an $\mathcal{A}_{1}$-valued inner product $\langle\cdot, \cdot\rangle_{\mathcal{A}_{1}}$, and a left Hilbert $\mathcal{A}_{2}$-module with an $\mathcal{A}_{2}$-valued inner product $\langle\cdot, \cdot\rangle_{\mathcal{A}_{2}}$, such that we have:

- We obtain all $\mathcal{A}_{i}$ as the closure of the span of

$$
\left\{\left\langle\xi_{1}, \xi_{2}\right\rangle_{\mathcal{A}_{i}}: \xi_{1}, \xi_{2} \in \mathcal{M}\right\}
$$

- $\forall \xi_{1}, \xi_{2}, \xi_{3} \in \mathcal{M}$ we have

$$
\left\langle\xi_{1}, \xi_{2}\right\rangle_{\mathcal{A}_{1}} \xi_{3}=\xi_{1}\left\langle\xi_{2}, \xi_{3}\right\rangle_{\mathcal{A}_{2}}
$$

- $\mathcal{A}_{1}$ and $\mathcal{A}_{2}$ act on $\mathcal{M}$ by bounded operators,

$$
\begin{aligned}
& \left\langle a_{2} \xi, a_{2} \xi\right\rangle_{\mathcal{A}_{1}} \leq\left\|a_{2}\right\|^{2}\langle\xi, \xi\rangle_{\mathcal{A}_{1}} \quad\left\langle a_{1} \xi, a_{1} \xi\right\rangle_{\mathcal{A}_{2}} \leq\left\|a_{1}\right\|^{2}\langle\xi, \xi\rangle_{\mathcal{A}_{2}} \\
& \quad \text { for all } a_{1} \in \mathcal{A}_{1}, a_{2} \in \mathcal{A}_{2}, \xi \in \mathcal{M} .
\end{aligned}
$$

This notion of equivalence roughly means that one can transfer modules back and forth between the two algebras.

2.2. The tools of noncommutative geometry. Once one identifies in a specific problem a space that, by its nature of quotient of the type described above, is best described as a noncommutative space, there is a large set of well developed techniques that one can use to compute invariants and extract essential information from the geometry. The following is a list of some such techniques, some of which will make their appearance in the cases treated in these notes.

- Topological invariants: K-theory

- Hochschild and cyclic cohomology

- Homotopy quotients, assembly map (Baum-Connes)

- Metric structure: Dirac operator, spectral triples

- Characteristic classes, zeta functions

We will recall the necessary notions when needed. We now begin by taking a closer look at the analog in the noncommutative world of Riemannian geometry, which is provided by Connes' notion of spectral triples. 


\section{Spectral triples}

Spectral triples are a crucial notion in noncommutative geometry. They provide a powerful and flexible generalization of the classical structure of a Riemannian manifold. The two notions agree on a commutative space. In the usual context of Riemannian geometry, the definition of the infinitesimal element $d s$ on a smooth spin manifold can be expressed in terms of the inverse of the classical Dirac operator $D$. This is the key remark that motivates the theory of spectral triples. In particular, the geodesic distance between two points on the manifold is defined in terms of $D^{-1}(c f$. [21] $\S \mathrm{VI})$. The spectral triple $(A, H, D)$ that describes a classical Riemannian spin manifold is given by the algebra $A$ of complex valued smooth functions on the manifold, the Hilbert space $H$ of square integrable spinor sections, and the classical Dirac operator $D$. These data determine completely and uniquely the Riemannian geometry on the manifold. It turns out that, when expressed in this form, the notion of spectral triple extends to more general non-commutative spaces, where the data $(A, H, D)$ consist of a $\mathrm{C}^{*}$-algebra $A$ (or more generally of some smooth subalgebra of a $\mathrm{C}^{*}$ algebra) with a representation in the algebra of bounded operators on a separable Hilbert space $H$, and an operator $D$ on $H$ that verifies the main properties of a Dirac operator.

We recall the basic setting of Connes' theory of spectral triples. For a more complete treatment see $[\mathbf{2 1}],[\mathbf{2 2}],[39]$.

Definition 3.1. A spectral triple $(\mathcal{A}, \mathcal{H}, D)$ consists of a $\mathrm{C}^{*}$-algebra $\mathcal{A}$ with a representation

$$
\rho: \mathcal{A} \rightarrow \mathcal{B}(\mathcal{H})
$$

in the algebra of bounded operators on a separable Hilbert space $\mathcal{H}$, and an operator $D$ (called the Dirac operator) on $\mathcal{H}$, which satisfies the following properties:

(1) $D$ is self-adjoint.

(2) For all $\lambda \notin \mathbb{R}$, the resolvent $(D-\lambda)^{-1}$ is a compact operator on $\mathcal{H}$.

(3) The commutator $[D, \rho(a)]$ is a bounded operator on $\mathcal{H}$, for all $a \in \mathcal{A}_{0} \subset \mathcal{A}$, a dense involutive subalgebra of $\mathcal{A}$.

The property (2) of Definition 3.1 can be regarded as a generalization of the ellipticity property of the standard Dirac operator on a compact manifold. In the case of ordinary manifolds, we can consider as subalgebra $\mathcal{A}_{0}$ the algebra of smooth functions, as a subalgebra of the commutative $\mathrm{C}^{*}$-algebra of continuous functions. In fact, in the 
classical case of Riemannian manifolds, property (3) is equivalent the Lipschitz condition, hence it is satisfied by a larger class than that of smooth functions.

Thus, the basic geometric structure encoded by the theory of spectral triples is Riemannian geometry, but in more refined cases, such as Kähler geometry, the additional structure can be easily encoded as additional symmetries. We will see, for instance, a case (cf. [44] [48]) where the algebra involves the action of the Lefschetz operator of a compact Kähler manifold, hence it encodes the information (at the cohomological level) on the Kähler form.

Since we are mostly interested in the relations between noncommutative geometry and arithmetic geometry and number theory, an especially interesting feature of spectral triples is that they have an associated family of zeta functions and a theory of volumes and integration, which is related to special values of these zeta functions. (The following treatment is based on $[\mathbf{2 1}],[\mathbf{2 2}]$.)

3.1. Volume form. A spectral triple $(\mathcal{A}, \mathcal{H}, D)$ is said to be of dimension $n$, or $n$-summable if the operator $|D|^{-n}$ is an infinitesimal of order one, which means that the eigenvalues $\lambda_{k}\left(|D|^{-n}\right)$ satisfy the estimate $\lambda_{k}\left(|D|^{-n}\right)=O\left(k^{-1}\right)$.

For a positive compact operator $T$ such that

$$
\sum_{j=0}^{k-1} \lambda_{j}(T)=O(\log k),
$$

the Dixmier trace $\operatorname{Tr}_{\omega}(T)$ is the coefficient of this logarithmic divergence, namely

$$
\operatorname{Tr}_{\omega}(T)=\lim _{\omega} \frac{1}{\log k} \sum_{j=1}^{k} \lambda_{j}(T) .
$$

Here the notation $\lim _{\omega}$ takes into account the fact that the sequence

$$
S(k, T):=\frac{1}{\log k} \sum_{j=1}^{k} \lambda_{j}(T)
$$

is bounded though possibly non-convergent. For this reason, the usual notion of limit is replaced by a choice of a linear form $\lim _{\omega}$ on the set of bounded sequences satisfying suitable conditions that extend analogous properties of the limit. When the sequence $S(k, T)$ converges (1.1) is just the ordinary limit $\operatorname{Tr}_{\omega}(T)=\lim _{k \rightarrow \infty} S(k, T)$. So defined, the Dixmier trace (1.1) extends to any compact operator that is an infinitesimal of order one, since any such operator is the difference of 
two positive ones. The operators for which the Dixmier trace does not depend on the choice of the linear form $\lim _{\omega}$ are called measurable operators.

On a non-commutative space the operator $|D|^{-n}$ generalizes the notion of a volume form. The volume is defined as

$$
V=\operatorname{Tr}_{\omega}\left(|D|^{-n}\right) .
$$

More generally, consider the algebra $\tilde{\mathcal{A}}$ generated by $\mathcal{A}$ and $[D, \mathcal{A}]$. Then, for $a \in \tilde{\mathcal{A}}$, integration with respect to the volume form $|D|^{-n}$ is defined as

$$
\int a:=\frac{1}{V} \operatorname{Tr}_{\omega}\left(a|D|^{-n}\right)
$$

The usual notion of integration on a Riemannian spin manifold $M$ can be recovered in this context $(c f .[\mathbf{2 1}],[\mathbf{7 0}])$ through the formula $(n$ even):

$$
\int_{M} f d v=\left(2^{n-[n / 2]-1} \pi^{n / 2} n \Gamma(n / 2)\right) \operatorname{Tr}_{\omega}\left(f|D|^{-n}\right) .
$$

Here $D$ is the classical Dirac operator on $M$ associated to the metric that determines the volume form $d v$, and $f$ in the right hand side is regarded as the multiplication operator acting on the Hilbert space of square integrable spinors on $M$.

3.2. Zeta functions. An important function associated to the Dirac operator $D$ of a spectral triple $(\mathcal{A}, \mathcal{H}, D)$ is its zeta function

$$
\zeta_{D}(z):=\operatorname{Tr}\left(|D|^{-z}\right)=\sum_{\lambda} \operatorname{Tr}(\Pi(\lambda,|D|)) \lambda^{-z},
$$

where $\Pi(\lambda,|D|)$ denotes the orthogonal projection on the eigenspace $E(\lambda,|D|)$.

An important result in the theory of spectral triples $([\mathbf{2 1}] \S I V$ Proposition 4) relates the volume (1.2) with the residue of the zeta function (1.4) at $s=1$ through the formula

$$
V=\lim _{s \rightarrow 1+}(s-1) \zeta_{D}(s)=\operatorname{Res}_{s=1} \operatorname{Tr}\left(|D|^{-s}\right) .
$$

There is a family of zeta functions associated to a spectral triple $(\mathcal{A}, \mathcal{H}, D)$, to which (1.4) belongs. For an operator $a \in \tilde{\mathcal{A}}$, we can define the zeta functions

$$
\zeta_{a, D}(z):=\operatorname{Tr}\left(a|D|^{-z}\right)=\sum_{\lambda} \operatorname{Tr}(a \Pi(\lambda,|D|)) \lambda^{-z}
$$


and

$$
\zeta_{a, D}(s, z):=\sum_{\lambda} \operatorname{Tr}(a \Pi(\lambda,|D|))(s-\lambda)^{-z} .
$$

These zeta functions are related to the heat kernel $e^{-t|D|}$ by Mellin transform

$$
\zeta_{a, D}(z)=\frac{1}{\Gamma(z)} \int_{0}^{\infty} t^{z-1} \operatorname{Tr}\left(a e^{-t|D|}\right) d t
$$

where

$$
\operatorname{Tr}\left(a e^{-t|D|}\right)=\sum_{\lambda} \operatorname{Tr}(a \Pi(\lambda,|D|)) e^{-t \lambda}=: \theta_{a, D}(t) .
$$

Similarly,

$$
\zeta_{a, D}(s, z)=\frac{1}{\Gamma(z)} \int_{0}^{\infty} \theta_{a, D, s}(t) t^{z-1} d t
$$

with

$$
\theta_{a, D, s}(t):=\sum_{\lambda} \operatorname{Tr}(a \Pi(\lambda,|D|)) e^{(s-\lambda) t} .
$$

Under suitable hypothesis on the asymptotic expansion of (1.11) (cf. Theorem 2.7-2.8 of [78] §2), the functions (1.6) and (1.7) admit a unique analytic continuation $(c f .[39])$ and there is an associated regularized determinant in the sense of Ray-Singer ( $c f .[\mathbf{1 0 0}])$ :

$$
\operatorname{det}_{\infty}(s):=\exp \left(-\left.\frac{d}{d z} \zeta_{a, D}(s, z)\right|_{z=0}\right) .
$$

The family of zeta functions (1.6) also provides a refined notion of dimension for a spectral triple $(\mathcal{A}, \mathcal{H}, D)$, called the dimension spectrum. This is a subset $\Sigma=\Sigma(\mathcal{A}, \mathcal{H}, D)$ in $\mathbb{C}$ with the property that all the zeta functions (1.6), as a varies in $\tilde{\mathcal{A}}$, extend holomorphically to $\mathbb{C} \backslash \Sigma$.

Examples of spectral triples with dimension spectrum not contained in the real line can be constructed out of Cantor sets.

3.3. Index map. The data of a spectral triple determine an index map. In fact, the self-adjoint operator $D$ has a polar decomposition, $D=F|D|$, where $|D|=\sqrt{D^{2}}$ is a positive operator and $F$ is a sign operator, i.e. $F^{2}=I$. Following $[\mathbf{2 1}]$ and [22] one defines a cyclic cocycle

$$
\tau\left(a^{0}, a^{1}, \ldots, a^{n}\right)=\operatorname{Tr}\left(a^{0}\left[F, a^{1}\right] \cdots\left[F, a^{n}\right]\right),
$$

where $n$ is the dimension of the spectral triple. For $n$ even Tr should be replaced by a super trace, as usual in index theory. This cocycle 
pairs with the $K$-groups of the algebra $\mathcal{A}$ (with $K_{0}$ in the even case and with $K_{1}$ in the odd case), and defines a class $\tau$ in the cyclic cohomology $H C^{n}(\mathcal{A})$ of $\mathcal{A}$. The class $\tau$ is called the Chern character of the spectral triple $(\mathcal{A}, \mathcal{H}, D)$.

In the case when the spectral triple $(\mathcal{A}, \mathcal{H}, D)$ has discrete dimension spectrum $\Sigma$, there is a local formula for the cyclic cohomology Chern character (1.13), analogous to the local formula for the index in the commutative case. This is obtained by producing Hochschild representatives $(c f .[\mathbf{2 2}],[\mathbf{3 9}])$

$$
\varphi\left(a^{0}, a^{1}, \ldots, a^{n}\right)=\operatorname{Tr}_{\omega}\left(a^{0}\left[D, a^{1}\right] \cdots\left[D, a^{n}\right]|D|^{-n}\right) .
$$

3.4. Infinite dimensional geometries. The main difficulty in constructing specific examples of spectral triples is to produce an operator $D$ that at the same time has bounded commutators with the elements of $\mathcal{A}$ and produces a non-trivial index map.

It sometimes happens that a noncommutative space does not admit a finitely summable spectral triple, namely one such that the operator $|D|^{-p}$ is of trace class for some $p>0$. Obstructions to the existence of such spectral triples are analyzed in $[\mathbf{2 0}]$. It is then useful to consider a weaker notion, namely that of $\theta$-summable spectral triples. These have the property that the Dirac operator satisfies

$$
\operatorname{Tr}\left(e^{-t D^{2}}\right)<\infty \quad \forall t>0 .
$$

Such spectral triples should be thought of as "infinite dimensional noncommutative geometries".

We'll see examples of spectral triples that are $\theta$-summable but not finitely summable, because of the growth rate of the multiplicities of eigenvalues.

3.5. Spectral triples and Morita equivalences. If $\left(\mathcal{A}_{1}, \mathcal{H}, D\right)$ is a spectral triple, and we have a Morita equivalence $\mathcal{A}_{1} \sim \mathcal{A}_{2}$ implemented by a bimodule $\mathcal{M}$ which is a finite projective right Hilbert module over $\mathcal{A}_{1}$, then we can transfer the spectral triple from $\mathcal{A}_{1}$ to $\mathcal{A}_{2}$.

First consider the $\mathcal{A}_{1}$-bimodule $\Omega_{D}^{1}$ generated by

$$
\left\{a_{1}\left[D, b_{1}\right]: a_{1}, b_{1} \in \mathcal{A}_{1}\right\} .
$$

We define a connection

$$
\nabla: \mathcal{M} \rightarrow \mathcal{M} \otimes_{\mathcal{A}_{1}} \Omega_{D}^{1}
$$

by requiring that

$$
\nabla\left(\xi a_{1}\right)=(\nabla \xi) a_{1}+\xi \otimes\left[D, a_{1}\right]
$$


$\forall \xi \in \mathcal{M}, \forall a_{1} \in \mathcal{A}_{1}$ and $\forall \xi_{1}, \xi_{2} \in \mathcal{M}$. We also require that

$$
\left\langle\xi_{1}, \nabla \xi_{2}\right\rangle_{\mathcal{A}_{1}}-\left\langle\nabla \xi_{1}, \xi_{2}\right\rangle_{\mathcal{A}_{1}}=\left[D,\left\langle\xi_{1}, \xi_{2}\right\rangle_{\mathcal{A}_{1}}\right] .
$$

This induces a spectral triple $\left(\mathcal{A}_{2}, \tilde{\mathcal{H}}, \tilde{D}\right)$ obtained as follows.

The Hilbert space is given by $\tilde{\mathcal{H}}=\mathcal{M} \otimes_{\mathcal{A}_{1}} \mathcal{H}$. The action takes the form

$$
a_{2}\left(\xi \otimes_{\mathcal{A}_{1}} x\right):=\left(a_{2} \xi\right) \otimes_{\mathcal{A}_{1}} x .
$$

The Dirac operator is given by

$$
\tilde{D}(\xi \otimes x)=\xi \otimes D(x)+(\nabla \xi) x .
$$

Notice that we need a Hermitian connection $\nabla$, because commutators $[D, a]$ for $a \in \mathcal{A}_{1}$ are non-trivial, hence $1 \otimes D$ would not be well defined on $\tilde{\mathcal{H}}$.

3.6. K-theory of $C^{*}$-algebras. The $K$-groups are important invariants of $C^{*}$-algebras that capture information on the topology of non-commutative spaces, much like cohomology (or more appropriately topological $K$-theory) captures information on the topology of ordinary spaces. For a $C^{*}$-algebra $\mathcal{A}$, we have:

3.6.1. $K_{0}(\mathcal{A})$. This group is obtained by considering idempotents $\left(p^{2}=p\right)$ in matrix algebras over $\mathcal{A}$. Notice that, for a $C^{*}$-algebra, it is sufficient to consider projections $\left(P^{2}=P, P=P^{*}\right)$. In fact, we can always replace an idempotent $p$ by a projection $P=p p^{*}(1-(p-$ $\left.\left.p^{*}\right)^{2}\right)^{-1}$, preserving the von Neumann equivalence. Then we consider $P \simeq Q$ if and only if $P=X^{*} X, Q=X X^{*}$, for $X=$ partial isometry $\left(X=X X^{*} X\right)$, and we impose the stable equivalence: $P \sim Q$, for $P \in \mathcal{M}_{n}(\mathcal{A})$ and $Q \in \mathcal{M}_{m}(\mathcal{A})$, if and only if there exists $R$ a projection with $P \oplus R \simeq Q \oplus R$. We define $K_{0}(\mathcal{A})^{+}$to be the monoid of projections modulo these equivalences and we let $K_{0}(\mathcal{A})$ be its Grothendieck group.

3.6.2. $K_{1}(\mathcal{A})$. Consider the group $\mathrm{GL}_{n}(\mathcal{A})$ of invertible elements in the matrix algebra $\mathcal{M}_{n}(\mathcal{A})$, and $\mathrm{GL}_{n}^{0}(\mathcal{A})$ the identity component of $\mathrm{GL}_{n}(\mathcal{A})$. The morphism

$$
\mathrm{GL}_{n}(\mathcal{A}) \rightarrow \mathrm{GL}_{n+1}(\mathcal{A}) \quad a \mapsto\left(\begin{array}{cc}
a & 0 \\
0 & 1
\end{array}\right)
$$

induces

$$
\mathrm{GL}_{n}(\mathcal{A}) / \mathrm{GL}_{n}^{0}(\mathcal{A}) \rightarrow \mathrm{GL}_{n+1}(\mathcal{A}) / \mathrm{GL}_{n+1}^{0}(\mathcal{A}) .
$$

The group $K_{1}(\mathcal{A})$ is defined as the direct limit of these morphisms. Notice that $K_{1}(\mathcal{A})$ is abelian even if the $\operatorname{GL}_{n}(\mathcal{A}) / \mathrm{GL}_{n}^{0}(\mathcal{A})$ are not.

In general, $K$-theory is not easy to compute. This has led to two fundamental developments in noncommutative geometry. The first is cyclic cohomology ( $c f .[\mathbf{1 8}]$, [19], [21]), introduced by Connes in 1981, 
which provides cycles that pair with $K$-theory, and a Chern character. The second development is the geometrically defined $K$-theory of Baum-Connes [7] and the assembly map

$$
\mu: K^{*}(X, G) \rightarrow K_{*}\left(C_{0}(X) \rtimes G\right)
$$

from the geometric to the analytic $K$-theory. Much work has gone, in recent years, into exploring the range of validity of the Baum-Connes conjecture, according to which the assembly map gives an isomorphism between these two different notions of $K$-theory.

\section{Why noncommutative geometry?}

In this short introductory section about noncommutative geometry we have tried to stress the idea that this version of geometry allows one to progress beyond the limits of applicability of ordinary "classical" geometry. Typically, this is the type of situation that presents itself at the boundary of classically defined objects, where certain quotients become ill behaved from the point of view of classical geometry. In such cases, by viewing the resulting spaces as noncommutative, which means working with a noncommutative algebra of coordinates replacing the usual commutative ring of functions of a space, one can continue to apply the tools and results of ordinary geometry.

Hopefully, this constitutes a convincing explanation of why one is led naturally to consider this type of geometry in a variety of contexts that have to do with boundary strata of certain classical objects and in general with equivalence relations that stem from suitably enlarging the class of objects to include some degenerate cases (all this seems quite vague at this point, but much of the content of the following chapters will be concrete illustrations of this principle).

Still, it is probably a good idea to spend a few more words in justifying the use of noncommutative geometry specifically in the context of Number Theory and Arithmetic Algebraic Geometry, and what one expects from this new set of tools.

There are at present several directions in which the use of tools of noncommuative geometry in number theory may lead to interesting progress. One is the explicit class field theory problem. In this case, the results of Bost-Connes [9] showed that the Galois theory of the cyclotomic field $\mathbb{Q}^{a b}$ lives naturally as symmetries of zero temperature equilibrium states of a quantum statistical mechanical system. Recent work of Connes and the author showed that a similar noncommutative space with a natural time evolution gives rise to zero temperature equilibrium states whose symmetries are given by the automorphisms of the modular field. In joint work with Ramachandran, we showed how these 
constructions fit into a possible theory of noncommutative Shimura varieties, and we exhibited a related quantum statistical mechanical system that recovers the explicit class field theory for imaginary quadratic fields. This opens up a very concrete possibility that noncommutative geometry may be able to say something new about the real quadratic fields or other cases where the Hilbert 12th problem of explicit class field theory is not yet fully understood from a purely number theoretic viewpoint. Suggestive connections between the problem for real quadratic fields and noncommutative geometry were discussed by Manin in [75] and [76]. These results and perspectives are described in a chapter of this volume.

Another direction in which noncommutative geometry may be able to yield new results in number theory and arithmetic geometry is related to L-functions. This possibility was first brought to light by Connes' work on the spectral realization of the zeros of the Riemann zeta function and of $L$-functions with Grossencharakter [23]. His work provided a new approach to the generalized Riemann hypothesis through a Selberg trace formula for the action of the idèle class group on a noncommutative space obtained as a quotient (viewed in the sense discussed in this section) of the space of adèles. The noncommutative space involved in this construction is closely related (up to a duality) to the one of the Bost-Connes system. In a different direction, the recent work of Consani and the author showed that noncommutative techniques may help in describing the geometry of the fibers at infinity of arithmetic varieties and the correspnding Gamma factors contributing to the L-function. The latter results are described in the last chapter of this volume. There is reason to be optimistic that a combination of these approaches will lead to some new results about L-functions of arithmetic varieties.

Sometime noncommutative geometry leads to new conceptual explanations and points of view on classical number theoretic objects. A striking example is given by the modular Hecke algebras of Connes and Moscovici $[\mathbf{4 1}][\mathbf{4 2}]$ that recover and extend structures like the RankinCohen brackets of modular forms [110] in terms of Hopf algebra symmetries of noncommutative spaces and their Hopf cyclic cohomology. In a similar spirit, the results of Manin and the author discussed in the first chapter of this volume, recover arithmetic properties of modular curves from a noncommutative boundary, opening the possibility of similar constructions on the boundary of other moduli spaces of arithmetic significance. 
For the purpose of this monograph, we have made a selection of some recent results illustrating the interactions between noncommutative geometry and number theory/arithmetic geometry, reflecting some contributions of the author. In general, the subject is still a large "building site" in rapid evolution, but enough results have emerged so far to make the underlying vision look increasingly promising. At least, it seems to be a good time to start collecting the results and the perspectives they open and present them in a unified picture, which will outline the shape of the emerging landscape of what we may call "arithmetic noncommutative geometry". We hope that this book will succeed, at least in part, in this goal.

An acknowledgment. Many thanks are due to Alain Connes, Katia Consani, Yuri Manin, and Niranjan Ramachandran, whose contribution and insight gave so much to the work described in this monograph. I especially thank Yuri Manin for contributing a nice preface to this volume. I thank the universities of Toronto, Nottingham, and Vanderbilt for creating the occasion for the lecture series out of which this volume evolved. I thank Arthur Greenspoon for a careful proofreading of the manuscript. I also thank Ed Dunne for encouraging me to write all this in book form for the AMS. 


\section{CHAPTER 2}

\section{Noncommutative modular curves}

The results of this section are mostly based on the joint work of Yuri Manin and the author [83], with additional material from [84] and $[\mathbf{8 5}]$. We include necessary preliminary notions on modular curves and on noncommutative tori, respectively based on the papers [81] and [16], [101].

We first recall some aspects of the classical theory of modular curves. We will then show that these notions can be entirely recovered and enriched through the analysis of a noncommutative space associated to a compactification of modular curves, which includes noncommutative tori as possible degenerations of elliptic curves.

\section{Modular curves}

Let $G$ be a finite index subgroup of the modular group $\Gamma=\operatorname{PSL}(2, \mathbb{Z})$, and let $X_{G}$ denote the quotient

$$
X_{G}:=G \backslash \mathbb{H}^{2}
$$

where $\mathbb{H}^{2}$ is the 2-dimensional real hyperbolic plane, namely the upper half plane $\{z \in \mathbb{C}: \Im z>0\}$ with the metric $d s^{2}=|d z|^{2} /(\Im z)^{2}$. Equivalently, we identify $\mathbb{H}^{2}$ with the Poincaré disk $\{z:|z|<1\}$ with the metric $d s^{2}=4|d z|^{2} /\left(1-|z|^{2}\right)^{2}$.

We denote the quotient map by $\phi: \mathbb{H}^{2} \rightarrow X_{G}$.

Let $\mathbb{P}$ denote the coset space $\mathbb{P}:=\Gamma / G$. We can write the quotient $X_{G}$ equivalently as

$$
X_{G}=\Gamma \backslash\left(\mathbb{H}^{2} \times \mathbb{P}\right) .
$$

A tessellation of the hyperbolic plane $\mathbb{H}^{2}$ by fundamental domains for the action of $\operatorname{PSL}(2, \mathbb{Z})$ is illustrated in Figure 1 . The action of $\operatorname{PSL}(2, \mathbb{Z})$ by fractional linear transformations $z \mapsto \frac{a z+b}{c z+d}$ is usually written in terms of the generators $S: z \mapsto-1 / z$ and $T: z \mapsto z+1$ (inversion and translation). Equivalently, $\operatorname{PSL}(2, \mathbb{Z})$ can be identified with the free product $\operatorname{PSL}(2, \mathbb{Z}) \cong \mathbb{Z} / 2 * \mathbb{Z} / 3$, with generators $\sigma, \tau$ of order two and three, respectively given by $\sigma=S$ and $\tau=S T$. 


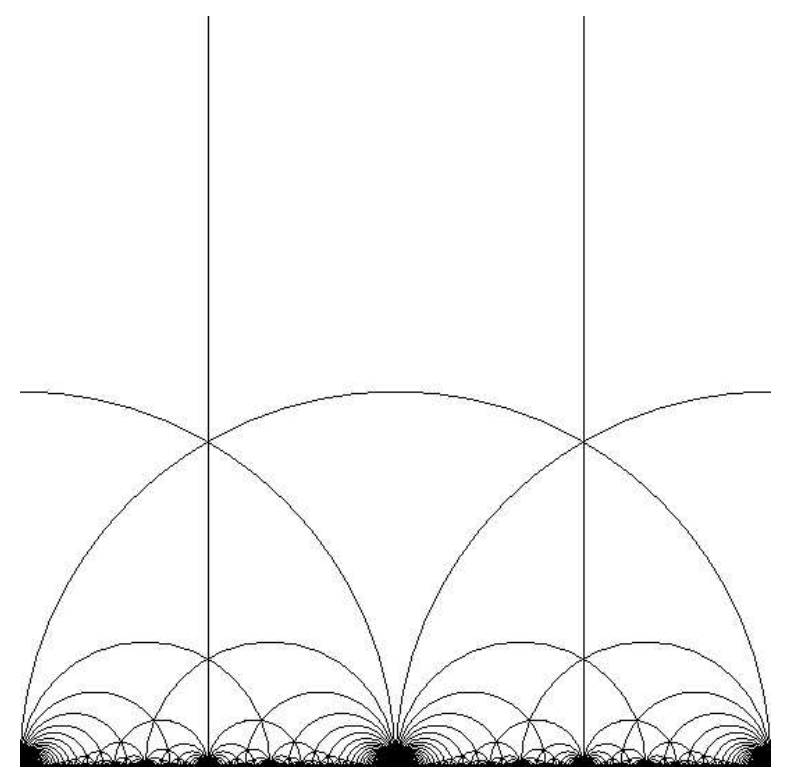

Figure 1. Fundamental domains for $\operatorname{PSL}(2, \mathbb{Z})$

An example of finite index subgroups is given by the congruence subgroups $\Gamma_{0}(N) \subset \Gamma$, of matrices

$$
\left(\begin{array}{ll}
a & b \\
c & d
\end{array}\right)
$$

with $c \equiv 0 \bmod N$. A fundamental domain for $\Gamma_{0}(N)$ is given by $F \cup_{k=0}^{N-1} S T^{k}(F)$, where $F$ is a fundamental domain for $\Gamma$ as in Figure $1^{1}$.

The quotient space $X_{G}$ has the structure of a non-compact Riemann surface. This has a natural algebro-geometric compactification, which consists of adding the cusp points (points at infinity). The cusp points are identified with the quotient

$$
G \backslash \mathbb{P}^{1}(\mathbb{Q}) \simeq \Gamma \backslash\left(\mathbb{P}^{1}(\mathbb{Q}) \times \mathbb{P}\right) .
$$

Thus, we write the compactification as

$$
\overline{X_{G}}:=G \backslash\left(\mathbb{H}^{2} \cup \mathbb{P}^{1}(\mathbb{Q})\right) \simeq \Gamma \backslash\left(\left(\mathbb{H}^{2} \cup \mathbb{P}^{1}(\mathbb{Q})\right) \times \mathbb{P}\right) .
$$

The modular curve $X_{\Gamma}$, for $\Gamma=\operatorname{SL}(2, \mathbb{Z})$ is the moduli space of elliptic curves, with the point $\tau \in \mathbb{H}^{2}$ parameterizing the lattice $\Lambda=$ $\mathbb{Z} \oplus \tau \mathbb{Z}$ in $\mathbb{C}$ and the corresponding elliptic curve uniformized by

$$
E_{\tau}=\mathbb{C} / \Lambda \text {. }
$$

\footnotetext{
${ }^{1}$ Figures 1 and 2 are taken from Curt McMullen's Gallery
} 
The unique cusp point corresponds to the degeneration of the elliptic curve to the cylinder $\mathbb{C}^{*}$, when $\tau \rightarrow \infty$ in the upper half plane.

The other modular curves, obtained as quotients $X_{G}$ by a congruence subgroup, can also be interpreted as moduli spaces: they are moduli spaces of elliptic curves with level structure. Namely, for elliptic curves $E=\mathbb{C} / \Lambda$, this is an additional information on the torsion points $\frac{1}{N} \Lambda / \Lambda \subset \mathbb{Q} \Lambda / \Lambda$ of some level $N$.

For instance, in the case of the principal congruence subgroups $\Gamma(N)$ of matrices

$$
M=\left(\begin{array}{ll}
a & b \\
c & d
\end{array}\right) \in \Gamma
$$

such that $M \equiv I d \bmod N$, points in the modular curve $\Gamma(N) \backslash \mathbb{H}^{2}$ classify elliptic curves $E_{\tau}=\mathbb{C} / \Lambda$, with $\Lambda=\mathbb{Z}+\mathbb{Z} \tau$, together with a basis $\{1 / N, \tau / N\}$ for the torsion subgroup $\frac{1}{N} \Lambda / \Lambda$. The projection $X_{\Gamma(N)} \rightarrow X_{\Gamma}$ forgets the extra structure.

In the case of the groups $\Gamma_{0}(N)$, points in the quotient $\Gamma_{0}(N) \backslash \mathbb{H}^{2}$ classify elliptic curves together with a cyclic subgroup of $E_{\tau}$ of order $N$. This extra information is equivalent to an isogeny $\phi: E_{\tau} \rightarrow E_{\tau^{\prime}}$ where the cyclic group is $\operatorname{Ker}(\phi)$. Recall that an isogeny is a morphism $\phi: E_{\tau} \rightarrow E_{\tau^{\prime}}$ such that $\phi(0)=0$. These are implemented by the action of $\mathrm{GL}_{2}^{+}(\mathbb{Q})$ on $\mathbb{H}^{2}$, namely $E_{\tau}$ and $E_{\tau^{\prime}}$ are isogenous if and only if $\tau$ and $\tau^{\prime}$ in $\mathbb{H}^{2}$ are in the same orbit of $\mathrm{GL}_{2}^{+}(\mathbb{Q})$.

1.1. Modular symbols. Given two points $\alpha, \beta \in \mathbb{H}^{2} \cup \mathbb{P}^{1}(\mathbb{Q})$, a real homology class $\{\alpha, \beta\}_{G} \in H_{1}\left(X_{G}, \mathbb{R}\right)$ is defined as

$$
\{\alpha, \beta\}_{G}: \omega \mapsto \int_{\alpha}^{\beta} \phi^{*}(\omega),
$$

where $\omega$ are holomorphic differentials on $X_{G}$, and the integration of the pullback to $\mathbb{H}^{2}$ is along the geodesic arc connecting two cusps $\alpha$ and $\beta$ (cf. Figure 2).

We consider here the modular symbols in $H_{1}\left(X_{G}, \mathbb{R}\right)$, though it was shown in [81] and [55] that in fact the functionals (2.4) really live in $H_{1}\left(X_{G}, \mathbb{Q}\right)$.

The modular symbols $\{\alpha, \beta\}_{G}$ satisfy the additivity and invariance properties

$$
\{\alpha, \beta\}_{G}+\{\beta, \gamma\}_{G}=\{\alpha, \gamma\}_{G},
$$

and

$$
\{g \alpha, g \beta\}_{G}=\{\alpha, \beta\}_{G},
$$

for all $g \in G$. 


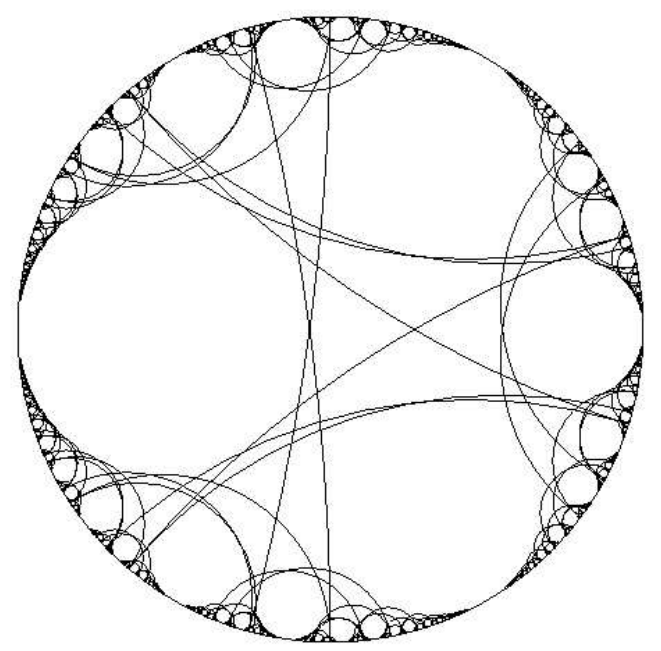

FiguRE 2. Geodesics between cusps define modular symbols

Because of additivity, it is sufficient to consider modular symbols of the form $\{0, \alpha\}$ with $\alpha \in \mathbb{Q}$,

$$
\{0, \alpha\}_{G}=-\sum_{k=1}^{n}\left\{g_{k}(\alpha) \cdot 0, g_{k}(\alpha) \cdot i \infty\right\}_{G},
$$

where $\alpha$ has continued fraction expansion $\alpha=\left[a_{0}, \ldots, a_{n}\right]$, and

$$
g_{k}(\alpha)=\left(\begin{array}{cc}
p_{k-1}(\alpha) & p_{k}(\alpha) \\
q_{k-1}(\alpha) & q_{k}(\alpha)
\end{array}\right)
$$

with $p_{k} / q_{k}$ the successive approximations, and $p_{n} / q_{n}=\alpha$.

In the classical theory of the modular symbols of $[\mathbf{8 1}],[\mathbf{9 0}]$ (cf. also [61], [89]) cohomology classes obtained from cusp forms are evaluated against relative homology classes given by modular symbols, namely, given a cusp form $\Phi$ on $\mathbb{H}$, obtained as pullback $\Phi=\varphi^{*}(\omega) / d z$ under the quotient map $\varphi: \mathbb{H} \rightarrow X_{G}$, we denote by $\Delta_{\omega}(s)$ the intersection numbers $\Delta_{\omega}(s)=\int_{g_{s}(0)}^{g_{s}(i \infty)} \Phi(z) d z$, with $g_{s} G=s \in \mathbb{P}$. These intersection numbers can be interpreted in terms of special values of $L$-functions associated to the automorphic form which determines the cohomology class.

We rephrase this in cohomological terms following [90]. We denote by $\tilde{I}$ and $\tilde{R}$ the elliptic points, namely the orbits $\tilde{I}=\Gamma \cdot i$ and $\tilde{R}=\Gamma \cdot \rho$. 
We denote by $I$ and $R$ the image in $X_{G}$ of the elliptic points

$$
I=G \backslash \tilde{I} \quad R=G \backslash \tilde{R},
$$

with $\rho=e^{\pi i / 3}$. We use the notation

$$
H_{A}^{B}:=H_{1}\left(\overline{X_{G}} \backslash A, B ; \mathbb{Z}\right) .
$$

These groups are related by the pairing

$$
H_{A}^{B} \times H_{B}^{A} \rightarrow \mathbb{Z}
$$

The modular symbols $\{g(0), g(i \infty)\}$, for $g G \in \mathbb{P}$, define classes in $H^{\text {cusps }}$. For $\sigma$ and $\tau$ the generators of $\operatorname{PSL}(2, \mathbb{Z})$ with $\sigma^{2}=1$ and $\tau^{3}=1$, we set

$$
\mathbb{P}_{I}=\langle\sigma\rangle \backslash \mathbb{P} \quad \text { and } \quad \mathbb{P}_{R}=\langle\tau\rangle \backslash \mathbb{P} .
$$

There is an isomorphism $\mathbb{Z}^{|\mathbb{P}|} \cong H_{\text {cusps } \cup I}^{R}$. Given the exact sequences

$$
0 \rightarrow H_{\text {cusps }} \stackrel{\iota^{\prime}}{\rightarrow} H_{\text {cusps }}^{R} \stackrel{\pi_{R}}{\rightarrow} \mathbb{Z}^{\left|\mathbb{P}_{R}\right|} \rightarrow \mathbb{Z} \rightarrow 0
$$

and

$$
0 \rightarrow \mathbb{Z}^{\left|\mathbb{P}_{I}\right|} \rightarrow H_{\text {cusps } \cup I}^{R} \stackrel{\pi_{I}}{\rightarrow} H_{\text {cusps }}^{R} \rightarrow 0,
$$

the image $\pi_{I}(\tilde{x}) \in H_{\text {cusps }}^{R}$ of an element $\tilde{x}=\sum_{s \in \mathbb{P}} \lambda_{s} s$ in $\mathbb{Z}^{|\mathbb{P}|} \cong H_{\text {cusps } \cup I}^{R}$ represents an element $x \in H_{\text {cusps }}$ iff the image $\pi_{R}\left(\pi_{I}(\tilde{x})\right)=0$ in $\mathbb{Z}^{\left|\mathbb{P}_{R}\right|}$. As proved in $[\mathbf{9 0}]$, for $s=g G \in \mathbb{P}$, the intersection pairing $\bullet: H^{\text {cusps }} \times$ $H_{\text {cusps }} \rightarrow \mathbb{Z}$ gives

$$
\{g(0), g(i \infty)\} \bullet x=\lambda_{s}-\lambda_{\sigma s} .
$$

Thus, we write the intersection number as a function $\Delta_{x}: \mathbb{P} \rightarrow \mathbb{R}$ by

$$
\Delta_{x}(s)=\lambda_{s}-\lambda_{\sigma s},
$$

where $x$ is given as above.

1.2. The modular complex. For $x$ and $y$ in $\mathbb{H}^{2}$ we denote by $\langle x, y\rangle$ the oriented geodesic arc connecting them. Moreover, in the decomposition $\operatorname{PSL}(2, \mathbb{Z})=\mathbb{Z} / 2 * \mathbb{Z} / 3$ as a free product, we denote by $\sigma$ the generator of $\mathbb{Z} / 2$ and by $\tau$ the generator of $\mathbb{Z} / 3$.

Definition 1.1. The modular complex is the cell complex defined as follows.

- 0-cells: the cusps $G \backslash \mathbb{P}^{1}(\mathbb{Q})$, and the elliptic points $I$ and $R$.

- 1-cells: the oriented geodesic arcs

$$
G \backslash(\Gamma \cdot\langle i \infty, i\rangle) \quad \text { and } \quad G \backslash(\Gamma \cdot\langle i, \rho\rangle),
$$

where by $\Gamma$. we mean the orbit under the action of $\Gamma$. 


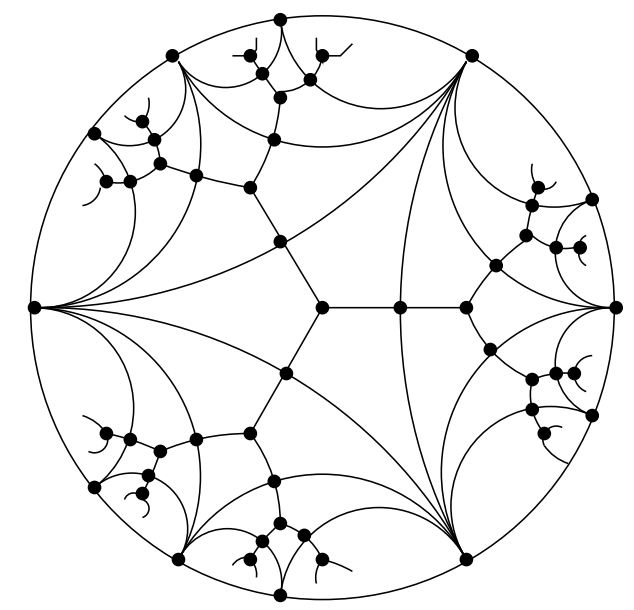

Figure 3. The cell decomposition of the modular complex

- 2-cells: $G \backslash\{\Gamma \cdot E\}$, where $E$ is the polygon with vertices

$$
\{i, \rho, 1+i, i \infty\}
$$

and sides the corresponding geodesic arcs.

- Boundary operator: $\partial: C_{2} \rightarrow C_{1}$ is given by

$$
g E \mapsto g\langle i, \rho\rangle+g\langle\rho, 1+i\rangle+g\langle 1+i, i \infty\rangle+g\langle i \infty, i\rangle,
$$

for $g \in \Gamma$, and the boundary $\partial: C_{1} \rightarrow C_{0}$ is given by

$$
\begin{gathered}
g\langle i \infty, i\rangle \mapsto g(i)-g(i \infty) \\
g\langle i, \rho\rangle \mapsto g(\rho)-g(i) .
\end{gathered}
$$

This gives a cell decomposition of $\mathbb{H}^{2}$ adapted to the action of $\operatorname{PSL}(2, \mathbb{Z})$ and congruence subgroups, $c f$. Figure 3.

We have the following result $[\mathbf{8 1}]$.

Proposition 1.1. The modular complex computes the first homology of $\overline{X_{G}}$ :

$$
H_{1}\left(X_{G}\right) \cong \frac{\operatorname{Ker}\left(\partial: C_{1} \rightarrow C_{0}\right)}{\operatorname{Im}\left(\partial: C_{2} \rightarrow C_{1}\right)} .
$$

We can derive versions of the modular complex that compute relative homology.

Notice that we have $\mathbb{Z}[$ cusps $]=C_{0} / \mathbb{Z}[R \cup I]$, hence the quotient complex

$$
0 \rightarrow C_{2} \stackrel{\partial}{\rightarrow} C_{1} \stackrel{\tilde{\partial}}{\rightarrow} \mathbb{Z}[\text { cusps }] \rightarrow 0
$$


with $\tilde{\partial}$ the induced boundary operator, computes the relative homology $H_{1}\left(\overline{X_{G}}, R \cup I\right)$. The cycles are given by $\mathbb{Z}[\mathbb{P}]$, as combinations of elements $g\langle i, \rho\rangle, g$ ranging over representatives of $\mathbb{P}$, and by the elements $\oplus a_{g}\langle g(i \infty), g(i)\rangle$ satisfying $\sum a_{g} g(i \infty)=0$. In fact, these can be represented as relative cycles in $\left(\overline{X_{G}}, R \cup I\right)$. Similarly, the subcomplex

$$
0 \rightarrow \mathbb{Z}[\mathbb{P}] \stackrel{\partial}{\rightarrow} \mathbb{Z}[R \cup I] \rightarrow 0,
$$

with $\mathbb{Z}[\mathbb{P}]$ generated by the elements $g\langle i, \rho\rangle$, computes the homology $H_{1}\left(\overline{X_{G}}-\right.$ cusps $)$. The homology

$$
H_{1}\left(\overline{X_{G}}-\text { cusps, } R \cup I\right) \cong \mathbb{Z}[\mathbb{P}]
$$

is generated by the relative cycles $g\langle i, \rho\rangle, g$ ranging over representatives of $\mathbb{P}$.

With the notation (2.5), we consider the groups $H_{R \cup I}^{\text {cusps }}, H_{\text {cusps }}^{R \cup I}$, $H^{\text {cusps }}$, and $H_{\text {cusps }}$.

We have a long exact sequence of relative homology

$$
0 \rightarrow H_{\text {cusps }} \rightarrow H_{\text {cusps }}^{R \cup I} \stackrel{\left(\tilde{\beta}_{R}, \tilde{\beta}_{I}\right)}{\rightarrow} H_{0}(R) \oplus H_{0}(I) \rightarrow \mathbb{Z} \rightarrow 0,
$$

with $H_{\text {cusps }}$ and $H_{\text {cusps }}^{R \cup I}$ as above, and with

$$
\begin{array}{cc}
H_{0}(I) \cong \mathbb{Z}\left[\mathbb{P}_{I}\right], \quad \mathbb{P}_{I}=\langle\sigma\rangle \backslash \mathbb{P}=G \backslash \tilde{I} \\
H_{0}(R) \cong \mathbb{Z}\left[\mathbb{P}_{R}\right], \quad \mathbb{P}_{R}=\langle\tau\rangle \backslash \mathbb{P}=G \backslash \tilde{R},
\end{array}
$$

that is,

$$
0 \rightarrow H_{\text {cusps }} \rightarrow \mathbb{Z}[\mathbb{P}] \rightarrow \mathbb{Z}\left[\mathbb{P}_{R}\right] \oplus \mathbb{Z}\left[\mathbb{P}_{I}\right] \rightarrow \mathbb{Z} \rightarrow 0 .
$$

In the case of $H_{\text {cusps }}^{R \cup I}$ and $H_{R \cup I}^{\text {cusps }}$ the pairing (2.6) gives the identification of $\mathbb{Z}[\mathbb{P}]$ and $\mathbb{Z}^{|\mathbb{P}|}$, obtained by identifying the elements of $\mathbb{P}$ with the corresponding delta functions. Thus, we can rewrite the sequence (2.11) as

$$
0 \rightarrow H^{\text {cusps }} \rightarrow \mathbb{Z}^{|\mathbb{P}|} \stackrel{\left(\beta_{R}, \beta_{I}\right)}{\rightarrow} \mathbb{Z}^{\left|\mathbb{P}_{I}\right|} \oplus \mathbb{Z}^{\left|\mathbb{P}_{R}\right|} \rightarrow \mathbb{Z} \rightarrow 0,
$$

where $H_{R \cup I}^{\text {cusps }} \cong \mathbb{Z}^{|\mathbb{P}|}$.

In order to understand more explicitly the map $\left(\beta_{R}, \beta_{I}\right)$ we give the following equivalent algebraic formulation of the modular complex ( $c f$. [81] [90]).

The homology group $H_{\text {cusps }}^{R \cup I}=\mathbb{Z}[\mathbb{P}]$ is generated by the images in $X_{G}$ of the geodesic segments $g \gamma_{0}:=g\langle i, \rho\rangle$, with $g$ ranging over a chosen set of representatives of the coset space $\mathbb{P}$.

We can identify $(c f .[\mathbf{9 0}])$ the dual basis $\delta_{s}$ of $H_{R \cup I}^{\text {cusps }}=\mathbb{Z}^{|\mathbb{P}|}$ with the images in $X_{G}$ of the paths $g \eta_{0}$, where for a chosen point $z_{0}$ with 
$0<\Re\left(z_{0}\right)<1 / 2$ and $\left|z_{0}\right|>1$ the path $\eta_{0}$ is given by the geodesic arcs connecting $\infty$ to $z_{0}, z_{0}$ to $\tau z_{0}$, and $\tau z_{0}$ to 0 . These satisfy

$$
\begin{gathered}
{\left[g \gamma_{0}\right] \bullet\left[g \eta_{0}\right]=1} \\
{\left[g \gamma_{0}\right] \bullet\left[h \eta_{0}\right]=0,}
\end{gathered}
$$

for $g G \neq h G$, under the intersection pairing (2.6)

Then, in the exact sequence (2.12), the identification of $H^{\text {cusps }}$ with $\operatorname{Ker}\left(\beta_{R}, \beta_{I}\right)$ is obtained by the identification $\{g(0), g(i \infty)\}_{G} \mapsto g \eta_{0}$, so that the relations imposed on the generators $\delta_{s}$ by the vanishing under $\beta_{I}$ correspond to the relations $\delta_{s} \oplus \delta_{\sigma s}$ (or $\delta_{s}$ if $s=\sigma s$ ) and the vanishing under $\beta_{R}$ gives another set of relations $\delta_{s} \oplus \delta_{\tau s} \oplus \delta_{\tau^{2} s}$ (or $\delta_{s}$ if $s=\tau s)$.

\section{The noncommutative boundary of modular curves}

The main idea that bridges between the algebro-geometric theory of modular curves and noncommutative geometry consists of replacing $\mathbb{P}^{1}(\mathbb{Q})$ in the classical compactification, which gives rise to a finite set of cusps, with $\mathbb{P}^{1}(\mathbb{R})$. This substitution cannot be done naively, since the quotient $G \backslash \mathbb{P}^{1}(\mathbb{R})$ is ill behaved topologically, as $G$ does not act discretely on $\mathbb{P}^{1}(\mathbb{R})$.

When we regard the quotient $\Gamma \backslash \mathbb{P}^{1}(\mathbb{R})$, or more generally a quotient $\Gamma \backslash\left(\mathbb{P}^{1}(\mathbb{R}) \times \mathbb{P}\right)$, itself as a noncommutative space, we obtain a geometric object that is rich enough to recover many important aspects of the classical theory of modular curves. In particular, it makes sense to study in terms of the geometry of such spaces the limiting behavior for certain arithmetic invariants defined on modular curves when $\tau \rightarrow \theta \in$ $\mathbb{R} \backslash \mathbb{Q}$.

2.1. Modular interpretation: noncommutative elliptic curves. The boundary $\Gamma \backslash \mathbb{P}^{1}(\mathbb{R})$ of the modular curve $\Gamma \backslash \mathbb{H}^{2}$, viewed itself as a noncommutative space, continues to have a modular interpretation, as observed originally by Connes-Douglas-Schwarz $([\mathbf{2 6}])$. In fact, we can think of the quotients of $S^{1}$ by the action of rotations by an irrational angle (that is, the noncommutative tori) as particular degenerations of the classical elliptic curves, which are "invisible" to ordinary algebraic geometry. The quotient space $\Gamma \backslash \mathbb{P}^{1}(\mathbb{R})$ classifies these noncommutative tori up to Morita equivalence $([\mathbf{1 6}],[\mathbf{1 0 1}])$ and completes the moduli space $\Gamma \backslash \mathbb{H}^{2}$ of the classical elliptic curves. Thus, from a conceptual point of view it is reasonable to think of $\Gamma \backslash \mathbb{P}^{1}(\mathbb{R})$ as the boundary of $\Gamma \backslash \mathbb{H}^{2}$, when we allow points in this classical moduli space (that is, elliptic curves) to have non-classical degenerations to noncommutative tori. 
Noncommutative tori are, in a sense, a prototype example of noncommutative spaces, inasmuch as one can see there displayed the full range of techniques of noncommutative geometry (cf. [16], [18]). As $\mathrm{C}^{*}$-algebras, noncommutative tori are irrational rotation algebras. We recall some basic properties of noncommutative tori, which justify the claim that these algebras behave like a noncommutative version of elliptic curves. We follow mostly [16] [21] and [101] for this material.

\subsection{Irrational rotation and Kronecker foliation.}

Definition 2.1. The irrational rotation algebra $\mathcal{A}_{\theta}$, for a given $\theta \in \mathbb{R}$, is the universal $\mathrm{C}^{*}$-algebra $\mathrm{C}^{*}(U, V)$, generated by two unitary operators $U$ and $V$, subject to the commutation relation

$$
U V=e^{2 \pi i \theta} V U
$$

The algebra $A_{\theta}$ can be realized as a subalgebra of bounded operators on the Hilbert space $\mathcal{H}=L^{2}\left(S^{1}\right)$, with the circle $S^{1} \cong \mathbb{R} / \mathbb{Z}$. For a given $\theta \in \mathbb{R}$, we consider two operators, that act on a complete orthonormal basis $e_{n}$ of $\mathcal{H}$ as

$$
U e_{n}=e_{n+1}, \quad V e_{n}=e^{2 \pi i n \theta} e_{n} .
$$

It is easy to check that these operators satisfy the commutation relation (2.13), since, for any $f \in \mathcal{H}$ we have $V U f(t)=U f(t-\theta)=$ $e^{2 \pi i(t-\theta)} f(t-\theta)$, while $U V f(t)=e^{2 \pi i t} f(t-\theta)$.

The irrational rotation algebra can be described in more geometric terms by the foliation on the usual commutative torus by lines with irrational slope. On $T^{2}=\mathbb{R}^{2} / \mathbb{Z}^{2}$ one considers the foliation $d x=\theta d y$, for $x, y \in \mathbb{R} / \mathbb{Z}$. The space of leaves is described as $X=\mathbb{R} /(\mathbb{Z}+\theta \mathbb{Z}) \simeq$ $S^{1} / \theta \mathbb{Z}$. This quotient is ill behaved as a classical topological space, hence it cannot be well described by ordinary geometry.

A transversal to the foliation is given for instance by the choice $T=\{y=0\}, T \cong S^{1} \cong \mathbb{R} / \mathbb{Z}$. Then the non-commutative torus is obtained $(c f .[\mathbf{1 6}][\mathbf{2 1}])$ as

$$
\mathcal{A}_{\theta}=\left\{\left(f_{a b}\right) \quad a, b \in T \text { in the same leaf }\right\}
$$

where $\left(f_{a b}\right)$ is a power series $b=\sum_{n \in \mathbb{Z}} b_{n} V^{n}$ and each $b_{n}$ is an element of the algebra $C\left(S^{1}\right)$. The multiplication is given by

$$
V h V^{-1}=h \circ R_{\theta}^{-1}
$$

with

$$
R_{\theta} x=x+\theta \bmod 1 .
$$




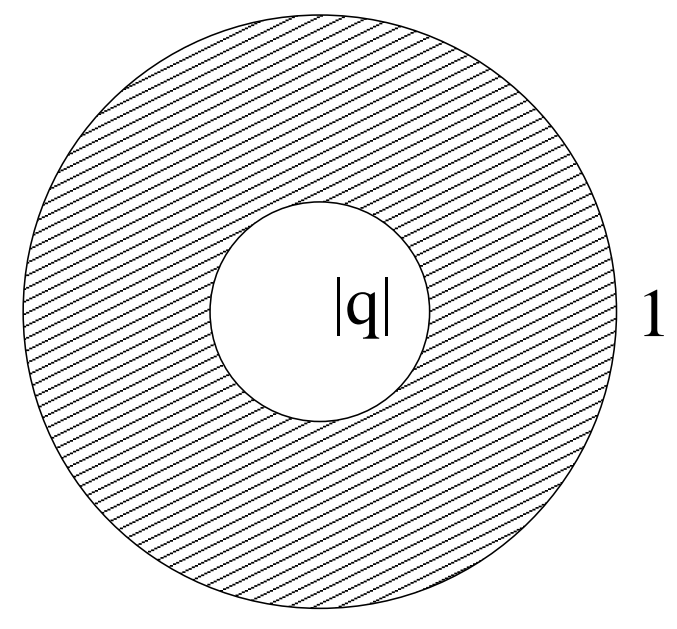

FiguRE 4. The fundamental domain for the Jacobi uniformization of the elliptic curve

The algebra $C\left(S^{1}\right)$ is generated by $U(t)=e^{2 \pi i t}$, hence we recover the generating system $(U, V)$ with the relation

$$
U V=e^{2 \pi i \theta} V U \text {. }
$$

What we have obtained through this description is an identification of the irrational rotation algebra of Definition 2.1 with the crossed product $C^{*}$-algebra

$$
\mathcal{A}_{\theta}=C\left(S^{1}\right) \rtimes_{R_{\theta}} \mathbb{Z}
$$

representing the quotient $S^{1} / \theta \mathbb{Z}$ as a non-commutative space.

2.3. Degenerations of elliptic curves. An elliptic curve $E_{\tau}$ over $\mathbb{C}$ can be described as the quotient $E_{\tau}=\mathbb{C} /(\mathbb{Z}+\tau \mathbb{Z})$ of the complex plane by a 2 -dimensional lattice $\Lambda=\mathbb{Z}+\tau \mathbb{Z}$, where we can take $\Im(\tau)>$ 0 . It is also possible to describe the elliptic curve $E_{q}$, for $q \in \mathbb{C}^{*}$, $q=\exp (2 \pi i \tau),|q|<1$, in terms of its Jacobi uniformization, namely as the quotient of $\mathbb{C}^{*}$ by the action of the group generated by a single hyperbolic element in $\operatorname{PSL}(2, \mathbb{C})$,

$$
E_{q}=\mathbb{C}^{*} / q^{\mathbb{Z}}
$$

The fundamental domain for the action of $q^{\mathbb{Z}}$ is an annulus

$$
\{z \in \mathbb{C}:|q|<z \leq 1\}
$$

of radii 1 and $|q|$, and the identification of the two boundary circles is obtained via the combination of scaling and rotation given by multiplication by $q$. 
Now let us consider a degeneration where $q \rightarrow \exp (2 \pi i \theta) \in S^{1}$, with $\theta \in \mathbb{R} \backslash \mathbb{Q}$. We can say heuristically that in this degeneration the elliptic curve becomes a non-commutative torus

$$
E_{q} \Longrightarrow \mathcal{A}_{\theta}
$$

in the sense that, as we let $q \rightarrow \exp (2 \pi i \theta)$, the annulus of the fundamental domain shrinks to a circle $S^{1}$ and we are left with a quotient of $S^{1}$ by the infinite cyclic group generated by the irrational rotation $\exp (2 \pi i \theta)$. Since this quotient is ill behaved as a classical quotient, such degenerations do not admit a description within the context of classical geometry. However, when we replace the quotient by the corresponding crossed product algebra $C\left(S^{1}\right) \rtimes_{\theta} \mathbb{Z}$ we find the irrational rotation algebra of definition 2.1. Thus, we can consider such algebras as non-commutative (degenerate) elliptic curves.

More precisely, when one considers degenerations of elliptic curves $E_{\tau}=\mathbb{C}^{*} / q^{\mathbb{Z}}$ for $q=e^{2 \pi i \tau}$, what one obtains in the limit is the suspension of a noncommutative torus. In fact, as the parameter $q$ degenerates to a point on the unit circle, $q \rightarrow e^{2 \pi i \theta}$, the "nice" quotient $E_{\tau}=\mathbb{C}^{*} / q^{\mathbb{Z}}$ degenerates to the "bad" quotient $E_{\theta}=\mathbb{C}^{*} / e^{2 \pi i \theta \mathbb{Z}}$, whose noncommutative algebra of coordinates is Morita equivalent to $\mathcal{A}_{\theta} \otimes C_{0}(\mathbb{R})$, with $\rho \in \mathbb{R}$ the radial coordinate, with $\mathbb{C}^{*} \ni z=e^{\rho} e^{2 \pi i s}$.

Because of the Thom isomorphism $[\mathbf{1 7}]$, the K-theory of the noncommutative space $E_{\theta}=C_{0}\left(\mathbb{R}^{2}\right) \rtimes(\mathbb{Z} \theta+\mathbb{Z})$ satisfies

$$
K_{0}\left(E_{\theta}\right)=K_{1}\left(\mathcal{A}_{\theta}\right) \text { and } K_{1}\left(E_{\theta}\right)=K_{0}\left(\mathcal{A}_{\theta}\right),
$$

which is again compatible with the identification of the $E_{\theta}$ (rather than $\mathcal{A}_{\theta}$ ) as degenerations of elliptic curves. In fact, for instance, the Hodge filtration on the $H^{1}$ of an elliptic curve and the equivalence between the elliptic curve and its Jacobian have analogs for the noncommutative torus $\mathcal{A}_{\theta}$ in terms of the filtration on $H C^{0}$ induced by the inclusion of $K_{0}$ (cf. [18] p. 132-139, [24] §XIII), while by the Thom isomorphism, these would again appear on the $H C^{1}$ in the case of the "noncommutative elliptic curve" $E_{\theta}$.

The point of view of degenerations is sometimes a useful guideline. For instance, one can study the limiting behavior of arithmetic invariants defined on the parameter space of elliptic curves (on modular curves), in the limit when $\tau \rightarrow \theta \in \mathbb{R} \backslash \mathbb{Q}$. An instance of this type of result is the theory of limiting modular symbols of [83], which we will review in this chapter.

2.4. Morita equivalent NC tori. To extend the modular interpretation of the quotient $\Gamma \backslash \mathbb{H}^{2}$ as moduli of elliptic curves to the noncommutative boundary $\Gamma \backslash \mathbb{P}^{1}(\mathbb{R})$, one needs to check that points 
in the same orbit of the action of the modular group $\operatorname{PSL}(2, \mathbb{Z})$ by fractional linear transformations on $\mathbb{P}^{1}(\mathbb{R})$ define equivalent noncommutative tori, where equivalence here is to be understood in the Morita sense.

Connes showed in $[\mathbf{1 6}]$ ( $c f$. also [101]) that the noncommutative tori $\mathcal{A}_{\theta}$ and $\mathcal{A}_{-1 / \theta}$ are Morita equivalent. Geometrically, in terms of the Kronecker foliation and the description (2.15) of the corresponding algebras, the Morita equivalence $\mathcal{A}_{\theta} \simeq \mathcal{A}_{-1 / \theta}$ corresponds to changing the choice of the transversal from $T=\{y=0\}$ to $T^{\prime}=\{x=0\}$.

In fact, all Morita equivalences arise in this way, by changing the choice of the transversal of the foliation, so that $\mathcal{A}_{\theta}$ and $\mathcal{A}_{\theta^{\prime}}$ are Morita equivalent if and only if $\theta \sim \theta^{\prime}$, under the action of $\operatorname{PSL}(2, \mathbb{Z})$.

Connes constructed in [16] explicit bimodules realizing the Morita equivalences between non-commutative tori $\mathcal{A}_{\theta}$ and $\mathcal{A}_{\theta^{\prime}}$ with

$$
\theta^{\prime}=\frac{a \theta+b}{c \theta+d}=g \theta
$$

for

$$
g=\left(\begin{array}{ll}
a & b \\
c & d
\end{array}\right) \in \Gamma
$$

by taking $\mathcal{M}_{\theta, \theta^{\prime}}$ to be the Schwartz space $\mathcal{S}(\mathbb{R} \times \mathbb{Z} / c)$, with the right action of $\mathcal{A}_{\theta}$

$$
\begin{gathered}
U f(x, u)=f\left(x-\frac{c \theta+d}{c}, u-1\right) \\
V f(x, u)=\exp (2 \pi i(x-u d / c)) f(x, u)
\end{gathered}
$$

and the left action of $\mathcal{A}_{\theta^{\prime}}$

$$
\begin{gathered}
U^{\prime} f(x, u)=f\left(x-\frac{1}{c}, u-a\right) \\
V^{\prime} f(x, u)=\exp \left(2 \pi i\left(\frac{x}{c \theta+d}-\frac{u}{c}\right)\right) f(x, u) .
\end{gathered}
$$

2.5. Other properties of NC elliptic curves. There are other ways in which the irrational rotation algebra behaves much like an elliptic curve, most notably the relation between the elliptic curve and its Jacobian ( $c f .[\mathbf{1 8}]$ and $[\mathbf{2 4}]$ ) and some aspects of the theory of theta functions, which we recall briefly.

The commutative torus $T^{2}=S^{1} \times S^{1}$ is connected, hence the algebra $\mathrm{C}\left(T^{2}\right)$ does not contain interesting projections. On the contrary, the noncommutative tori $\mathcal{A}_{\theta}$ contain a large family of nontrivial projections. Rieffel in [101] showed that, for a given $\theta$ irrational and for all $\alpha \in(\mathbb{Z} \oplus \mathbb{Z} \theta) \cap[0,1]$, there exists a projection $P_{\alpha}$ in $\mathcal{A}_{\theta}$, with $\operatorname{Tr}\left(P_{\alpha}\right)=\alpha$. 
A different construction of projections in $\mathcal{A}_{\theta}$, given by Boca [8], has arithmetic relevance, inasmuch as these projections correspond to the theta functions for noncommutative tori defined by Manin in [77].

A method of constructing projections in $C^{*}$-algebras is based on the following two steps $(c f .[\mathbf{1 0 1}]$ and $[\mathbf{7 6}])$ :

(1) Suppose given a bimodule ${ }_{\mathcal{A}} \mathcal{M}_{\mathcal{B}}$. If an element $\xi \in{ }_{\mathcal{A}} \mathcal{M}_{\mathcal{B}}$ admits an invertible $*$-invariant square root $\langle\xi, \xi\rangle_{\mathcal{B}}^{1 / 2}$, then the element $\mu:=\xi\langle\xi, \xi\rangle_{\mathcal{B}}^{-1 / 2}$ satisfies $\mu\langle\mu, \mu\rangle_{\mathcal{B}}=\mu$.

(2) Let $\mu \in{ }_{\mathcal{A}} \mathcal{M}_{\mathcal{B}}$ be a non-trivial element such that $\mu\langle\mu, \mu\rangle_{\mathcal{B}}=\mu$. Then the element $P:=_{\mathcal{A}}\langle\mu, \mu\rangle$ is a projection.

In Boca's construction, one obtains elements $\xi$ from Gaussian elements in some Heisenberg modules, in such a way that the corresponding $\langle\xi, \xi\rangle_{\mathcal{B}}$ is a quantum theta function in the sense of Manin [77]. An introduction to the relation between the Heisenberg groups and the theory of theta functions is given in the third volume of Mumford's Tata lectures on theta, $[\mathbf{9 2}]$.

\section{Limiting modular symbols}

We consider the action of the group $\Gamma=\operatorname{PGL}(2, \mathbb{Z})$ on the upper and lower half planes $\mathbb{H}^{ \pm}$and the modular curves defined by the quotient $X_{G}=G \backslash \mathbb{H}^{ \pm}$, for $G$ a finite index subgroup of $\Gamma$. Then the noncommutative compactification of the modular curves is obtained by extending the action of $\Gamma$ on $\mathbb{H}^{ \pm}$to the action on the full

$$
\mathbb{P}^{1}(\mathbb{C})=\mathbb{H}^{ \pm} \cup \mathbb{P}^{1}(\mathbb{R})
$$

so that we have

$$
\bar{X}_{G}=G \backslash \mathbb{P}^{1}(\mathbb{C})=\Gamma \backslash\left(\mathbb{P}^{1}(\mathbb{C}) \times \mathbb{P}\right) .
$$

Due to the fact that $\Gamma$ does not act discretely on $\mathbb{P}^{1}(\mathbb{R})$, the quotient (2.19) makes sense as a noncommutative space

$$
C\left(\mathbb{P}^{1}(\mathbb{C}) \times \mathbb{P}\right) \rtimes \Gamma .
$$

Here $\mathbb{P}^{1}(\mathbb{R}) \subset \mathbb{P}^{1}(\mathbb{C})$ is the limit set of the group $\Gamma$, namely the set of accumulation points of orbits of elements of $\Gamma$ on $\mathbb{P}^{1}(\mathbb{C})$. We will see another instance of noncommutative geometry arising from the action of a group of Möbius transformations of $\mathbb{P}^{1}(\mathbb{C})$ on its limit set, in the context of the geometry at the archimedean primes of arithmetic varieties. 
3.1. Generalized Gauss shift and dynamics. We have described the boundary of modular curves by the crossed product $C^{*}$ algebra

$$
C\left(\mathbb{P}^{1}(\mathbb{R}) \times \mathbb{P}\right) \rtimes \Gamma .
$$

We can also describe the quotient space $\Gamma \backslash\left(\mathbb{P}^{1}(\mathbb{R}) \times \mathbb{P}\right)$ in the following equivalent way. If $\Gamma=\operatorname{PGL}(2, \mathbb{Z})$, then $\Gamma$-orbits in $\mathbb{P}^{1}(\mathbb{R})$ are the same as equivalence classes of points of $[0,1]$ under the equivalence relation

$$
x \sim_{T} y \Leftrightarrow \exists n, m: T^{n} x=T^{m} y
$$

where $T x=1 / x-[1 / x]$ is the classical Gauss shift of the continued fraction expansion. Namely, the equivalence relation is that of having the same tail of the continued fraction expansion (shift-tail equivalence).

A simple generalization of this classical result yields the following.

LEMMA 3.1. $\Gamma$ orbits in $\mathbb{P}^{1}(\mathbb{R}) \times \mathbb{P}$ are the same as equivalence classes of points in $[0,1] \times \mathbb{P}$ under the equivalence relation

$$
(x, s) \sim_{T}(y, t) \Leftrightarrow \exists n, m: T^{n}(x, s)=T^{m}(y, t)
$$

where $T:[0,1] \times \mathbb{P} \rightarrow[0,1] \times \mathbb{P}$ is the shift

$$
T(x, s)=\left(\frac{1}{x}-\left[\frac{1}{x}\right],\left(\begin{array}{cc}
-[1 / x] & 1 \\
1 & 0
\end{array}\right) \cdot s\right)
$$

generalizing the classical shift of the continued fraction expansion.

As a noncommutative space, the quotient by the equivalence relation $(2.22)$ is described by the $C^{*}$-algebra of the groupoid of the equivalence relation

$$
\mathcal{G}([0,1] \times \mathbb{P}, T)=\left\{((x, s), m-n,(y, t)): T^{m}(x, s)=T^{n}(y, t)\right\}
$$

with objects $\mathcal{G}^{0}=\{((x, s), 0,(x, s))\}$.

In fact, for any $T$-invariant subset $E \subset[0,1] \times \mathbb{P}$, we can consider the equivalence relation (2.22). The corresponding groupoid $C^{*}$-algebra $C^{*}(\mathcal{G}(E, T))$ encodes the dynamical properties of the map $T$ on $E$.

Geometrically, the equivalence relation $(2.22)$ on $[0,1] \times \mathbb{P}$ is related to the action of the geodesic flow on the horocycle foliation on the modular curves.

3.2. Arithmetic of modular curves and noncommutative boundary. The result of Lemma 3.1 shows that the properties of the dynamical system $T$ or (2.22) can be used to describe the geometry of the noncommutative boundary of modular curves. There are various types of results that can be obtained by this method ([83] [84]), which we will discuss in the rest of this chapter. 
(1) Using the properties of this dynamical system it is possible to recover and enrich the theory of modular symbols on $X_{G}$, by extending the notion of modular symbols from geodesics connecting cusps to images of geodesics in $\mathbb{H}^{2}$ connecting irrational points on the boundary $\mathbb{P}^{1}(\mathbb{R})$. In fact, the irrational points of $\mathbb{P}^{1}(\mathbb{R})$ define limiting modular symbols. In the case of quadratic irrationalities, these can be expressed in terms of the classical modular symbols and recover the generators of the homology of the classical compactification by cusps $\overline{X_{G}}$. In the remaining cases, the limiting modular symbol vanishes almost everywhere.

(2) It is possible to reinterpret Dirichlet series related to modular forms of weight 2 in terms of integrals on $[0,1]$ of certain intersection numbers obtained from homology classes defined in terms of the dynamical system. In fact, even when the limiting modular symbol vanishes, it is possible to associate a non-trivial cohomology class in $X_{G}$ to irrational points on the boundary, in such a way that an average of the corresponding intersection numbers give Mellin transforms of modular forms of weight 2 on $X_{G}$.

(3) The Selberg zeta function of the modular curve can be expressed as a Fredholm determinant of the Perron-Frobenius operator associated to the dynamical system on the "boundary".

(4) Using the first formulation of the boundary as the noncommutative space (2.21) we can obtain a canonical identification of the modular complex with a sequence of K-groups of the $C^{*}$-algebra. The resulting exact sequence for K-groups can be interpreted, using the description (2.22) of the quotient space, in terms of the Baum-Connes assembly map and the Thom isomorphism.

All this shows that the noncommutative space $C\left(\mathbb{P}^{1}(\mathbb{R}) \times \mathbb{P}\right) \rtimes \Gamma$, which we have so far considered as a boundary stratum of $C\left(\mathbb{H}^{2} \times \mathbb{P}\right) \rtimes$ $\Gamma$, in fact contains a good part of the arithmetic information on the classical modular curve itself. The fact that information on the "bulk space" is stored in its boundary at infinity can be seen as an instance of the physical principle of holography (bulk/boundary correspondence) in string theory $(c f$. [82]). We will discuss the holography principle in more details in relation to the geometry of the archimedean fibers of arithmetic varieties. 
3.3. Limiting modular symbols. Let $\gamma_{\beta}$ be an infinite geodesic in the hyperbolic plane $\mathbb{H}$ with one end at $i \infty$ and the other end at $\beta \in \mathbb{R} \backslash \mathbb{Q}$. Let $x \in \gamma_{\beta}$ be a fixed base point, $\tau$ be the geodesic arc length, and $y(\tau)$ be the point along $\gamma_{\beta}$ at a distance $\tau$ from $x$, towards the end $\beta$. Let $\{x, y(\tau)\}_{G}$ denote the homology class in $X_{G}$ determined by the image of the geodesic arc $\langle x, y(\tau)\rangle$ in $\mathbb{H}$.

DeFinition 3.1. The limiting modular symbol is defined as

$$
\{\{*, \beta\}\}_{G}:=\lim \frac{1}{\tau}\{x, y(\tau)\}_{G} \in H_{1}\left(X_{G}, \mathbb{R}\right),
$$

whenever such limit exists.

The limit (2.24) is independent of the choice of the initial point $x$ as well as of the choice of the geodesic in $\mathbb{H}$ ending at $\beta$, as discussed in [83] (cf. Figure 5). We use the notation $\{\{*, \beta\}\}_{G}$ as introduced in [83], where $*$ in the first argument indicates the independence on the choice of $x$, and the double brackets indicate the fact that the homology class is computed as a limiting cycle.

3.3.1. Dynamics of continued fractions. As above, we consider on $[0,1] \times \mathbb{P}$ the dynamical system

$$
\begin{gathered}
T:[0,1] \times \mathbb{P} \rightarrow[0,1] \times \mathbb{P} \\
T(\beta, t)=\left(\frac{1}{\beta}-\left[\frac{1}{\beta}\right],\left(\begin{array}{cc}
-[1 / \beta] & 1 \\
1 & 0
\end{array}\right) \cdot t\right) .
\end{gathered}
$$

This generalizes the classical shift map of the continued fraction

$$
T:[0,1] \rightarrow[0,1] \quad T(x)=\frac{1}{x}-\left[\frac{1}{x}\right] .
$$

Recall the following basic notation regarding continued fraction expansion. Let $k_{1}, \ldots, k_{n}$ be independent variables and, for $n \geq 1$, let

$$
\left[k_{1}, \ldots, k_{n}\right]:=\frac{1}{k_{1}+\frac{1}{k_{2}+\ldots \frac{1}{k_{n}}}}=\frac{P_{n}\left(k_{1}, \ldots, k_{n}\right)}{Q_{n}\left(k_{1}, \ldots, k_{n}\right)} .
$$

The $P_{n}, Q_{n}$ are polynomials with integral coefficients, which can be calculated inductively from the relations

$$
\begin{gathered}
Q_{n+1}\left(k_{1}, \ldots, k_{n}, k_{n+1}\right)=k_{n+1} Q_{n}\left(k_{1}, \ldots, k_{n}\right)+Q_{n-1}\left(k_{1}, \ldots, k_{n-1}\right), \\
P_{n}\left(k_{1}, \ldots, k_{n}\right)=Q_{n-1}\left(k_{2}, \ldots, k_{n}\right),
\end{gathered}
$$

with $Q_{-1}=0, Q_{0}=1$. Thus, we obtain

$$
\left[k_{1}, \ldots, k_{n-1}, k_{n}+x_{n}\right]
$$




$$
=\frac{P_{n-1}\left(k_{1}, \ldots, k_{n-1}\right) x_{n}+P_{n}\left(k_{1}, \ldots, k_{n}\right)}{Q_{n-1}\left(k_{1}, \ldots, k_{n-1}\right) x_{n}+Q_{n}\left(k_{1}, \ldots, k_{n}\right)}=\left(\begin{array}{cc}
P_{n-1} & P_{n} \\
Q_{n-1} & Q_{n}
\end{array}\right)\left(x_{n}\right),
$$

with the standard matrix notation for fractional linear transformations,

$$
z \mapsto \frac{a z+b}{c z+d}=\left(\begin{array}{ll}
a & b \\
c & d
\end{array}\right)(z)
$$

If $\alpha \in(0,1)$ is an irrational number, there is a unique sequence of integers $k_{n}(\alpha) \geq 1$ such that $\alpha$ is the limit of $\left[k_{1}(\alpha), \ldots, k_{n}(\alpha)\right]$ as $n \rightarrow \infty$. Moreover, there is a unique sequence $x_{n}(\alpha) \in(0,1)$ such that

$$
\alpha=\left[k_{1}(\alpha), \ldots, k_{n-1}(\alpha), k_{n}(\alpha)+x_{n}(\alpha)\right]
$$

for each $n \geq 1$. We obtain

$$
\alpha=\left(\begin{array}{cc}
0 & 1 \\
1 & k_{1}(\alpha)
\end{array}\right) \ldots\left(\begin{array}{cc}
0 & 1 \\
1 & k_{n}(\alpha)
\end{array}\right)\left(x_{n}(\alpha)\right) .
$$

We set

$$
p_{n}(\alpha):=P_{n}\left(k_{1}(\alpha), \ldots, k_{n}(\alpha)\right), q_{n}(\alpha):=Q_{n}\left(k_{1}(\alpha), \ldots, k_{n}(\alpha)\right)
$$

so that $p_{n}(\alpha) / q_{n}(\alpha)$ is the sequence of convergents to $\alpha$. We also set

$$
g_{n}(\alpha):=\left(\begin{array}{cc}
p_{n-1}(\alpha) & p_{n}(\alpha) \\
q_{n-1}(\alpha) & q_{n}(\alpha)
\end{array}\right) \in \operatorname{GL}(2, \mathbb{Z})
$$

Written in terms of the continued fraction expansion, the shift $T$ is given by

$$
T:\left[k_{0}, k_{1}, k_{2}, \ldots\right] \mapsto\left[k_{1}, k_{2}, k_{3}, \ldots\right] .
$$

The properties of the shift (2.25) can be used to extend the notion of modular symbols to geodesics with irrational ends $([83])$. Such geodesics correspond to infinite geodesics on the modular curve $X_{G}$, which exhibit a variety of interesting possible behaviors, from closed geodesics to geodesics that approximate some limiting cycle, to geodesics that wind around different homology class exhibiting a typically chaotic behavior.

3.3.2. Lyapunov spectrum. A measure of how chaotic a dynamical system is, or better of how fast nearby orbits tend to diverge, is given by the Lyapunov exponent.

Definition 3.2. the Lyapunov exponent of $T:[0,1] \rightarrow[0,1]$ is defined as

$$
\lambda(\beta):=\lim _{n \rightarrow \infty} \frac{1}{n} \log \left|\left(T^{n}\right)^{\prime}(\beta)\right|=\lim _{n \rightarrow \infty} \frac{1}{n} \log \prod_{k=0}^{n-1}\left|T^{\prime}\left(T^{k} \beta\right)\right| .
$$


The function $\lambda(\beta)$ is $T$-invariant. Moreover, in the case of the classical continued fraction shift $T \beta=1 / \beta-[1 / \beta]$ on $[0,1]$, the Lyapunov exponent is given by

$$
\lambda(\beta)=2 \lim _{n \rightarrow \infty} \frac{1}{n} \log q_{n}(\beta),
$$

with $q_{n}(\beta)$ the successive denominators of the continued fraction expansion.

In particular, the Khintchine-Lévy theorem shows that, for almost all $\beta$ 's (with respect to the Lebesgue measure on $[0,1]$ ) the limit $(2.27$ ) is equal to

$$
\lambda(\beta)=\pi^{2} /(6 \log 2)=: \lambda_{0} .
$$

There is, however, an exceptional set in $[0,1]$ of Hausdorff dimension $\operatorname{dim}_{H}=1$ but with Lebesgue measure zero where the limit defining the Lyapunov exponent does not exist.

As we will see later, in "good cases" the value $\lambda(\beta)$ can be computed from the spectrum of the Perron-Frobenius operator of the shift $T$.

The Lyapunov spectrum is introduced (cf. [99]) by decomposing the unit interval into level sets of the Lyapunov exponent $\lambda(\beta)$ of $(2.26)$. Let $L_{c}=\{\beta \in[0,1] \mid \lambda(\beta)=c \in \mathbb{R}\}$. These sets provide a $T$-invariant decomposition of the unit interval,

$$
[0,1]=\bigcup_{c \in \mathbb{R}} L_{c} \cup\{\beta \in[0,1] \mid \lambda(\beta) \text { does not exist }\} .
$$

These level sets are uncountable dense $T$-invariant subsets of $[0,1]$, of varying Hausdorff dimension [99]. The Lyapunov spectrum measures how the Hausdorff dimension varies, as a function $h(c)=\operatorname{dim}_{H}\left(L_{c}\right)$.

3.3.3. Limiting modular symbols and iterated shifts. We introduce a function $\varphi: \mathbb{P} \rightarrow H^{\text {cusps }}$ of the form

$$
\varphi(s)=\{g(0), g(i \infty)\}_{G},
$$

where $g \in \operatorname{PGL}(2, \mathbb{Z})$ (or $\operatorname{PSL}(2, \mathbb{Z}))$ is a representative of the coset $s \in \mathbb{P}$.

Then we can compute the limit (2.24) in the following way.

TheOREM 3.2. Consider a fixed $c \in \mathbb{R}$ which corresponds to some level set $L_{c}$ of the Lyapunov exponent (2.27). Then, for all $\beta \in L_{c}$, the limiting modular symbol (2.24) is computed by the limit

$$
\lim _{n \rightarrow \infty} \frac{1}{c n} \sum_{k=1}^{n} \varphi \circ T^{k}\left(t_{0}\right),
$$




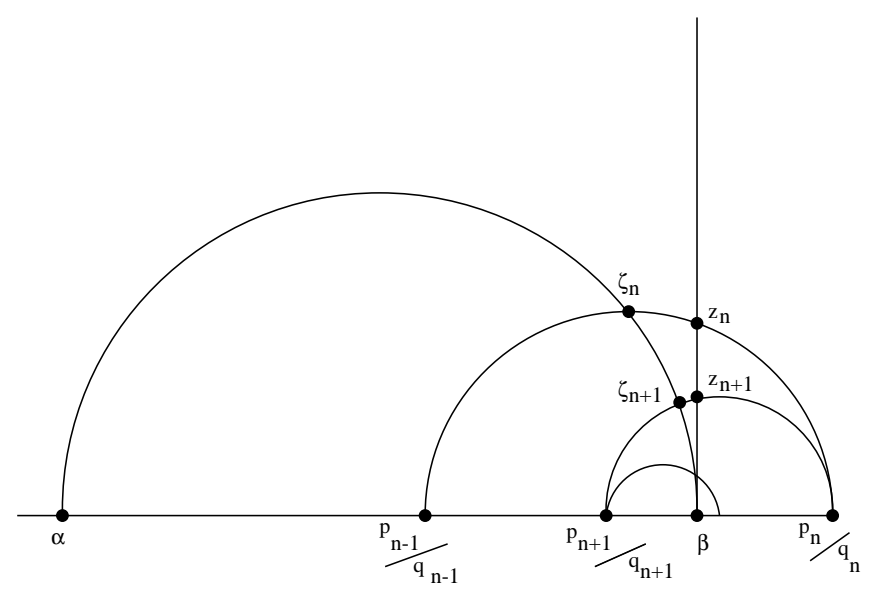

FiguRE 5. Geodesics defining limiting modular symbols

where $T$ is the shift of $(2.25)$ and $t_{0} \in \mathbb{P}$.

Without loss of generality, one can consider the geodesic $\gamma_{\beta}$ in $\mathbb{H} \times \mathbb{P}$ with one end at $\left(i \infty, t_{0}\right)$ and the other at $\left(\beta, t_{0}\right)$, for $\varphi\left(t_{0}\right)=\{0, i \infty\}_{G}$.

The argument given in $\S 2.3$ of [83] is based on the fact that one can replace the homology class defined by the vertical geodesic with one obtained by connecting the successive rational approximations to $\beta$ in the continued fraction expansion (Figure 5). Namely, one can replace the path $\left\langle x_{0}, y_{n}\right\rangle$ with the union of arcs

$$
\left\langle x_{0}, y_{0}\right\rangle \cup\left\langle y_{0}, p_{0} / q_{0}\right\rangle \cup \bigcup_{k=1}^{n}\left\langle p_{k-1} / q_{k-1}, p_{k} / q_{k}\right\rangle \cup\left\langle p_{n} / q_{n}, y_{n}\right\rangle
$$

representing the same homology class in $H_{1}\left(\bar{X}_{G}, \mathbb{Z}\right)$.

The result then follows by estimating the geodesic distance $\tau \sim$ $-\log \Im y+O(1)$, as $y(\tau) \rightarrow \beta$ and

$$
\frac{1}{2 q_{n} q_{n+1}}<\Im y_{n}<\frac{1}{2 q_{n} q_{n-1}}
$$

where $y_{n}$ is the intersection of $\gamma_{\beta}$ and the geodesic with ends at $p_{n-1}(\beta) / q_{n-1}(\beta)$ and $p_{n}(\beta) / q_{n}(\beta)$.

The matrix $g_{k}^{-1}(\beta)$, with

$$
g_{k}(\beta)=\left(\begin{array}{cc}
p_{k-1}(\beta) & p_{k}(\beta) \\
q_{k-1}(\beta) & q_{k}(\beta)
\end{array}\right)
$$


acts on points $(\beta, t) \in[0,1] \times \mathbb{P}$ as the $k$-th power of the shift map $T$ of (2.25). Thus, we obtain

$$
\varphi\left(T^{k} t_{0}\right)=\left\{g_{k}^{-1}(\beta)(0), g_{k}^{-1}(\beta)(i \infty)\right\}_{G}=\left\{\frac{p_{k-1}(\beta)}{q_{k-1}(\beta)}, \frac{p_{k}(\beta)}{q_{k}(\beta)}\right\}_{G} .
$$

3.4. Ruelle and Perron-Frobenius operators. A general principle in the theory of dynamical systems is that one can often study the dynamical properties of a map $T$ (e.g. ergodicity) via the spectral theory of an associated operator. This allows one to employ techniques of functional analysis and derive conclusions on dynamics.

In our case, to the shift map $T$ of (2.25), we associate the operator

$$
\left(L_{\sigma} f\right)(x, t)=\sum_{k=1}^{\infty} \frac{1}{(x+k)^{2 \sigma}} f\left(\frac{1}{x+k},\left(\begin{array}{ll}
0 & 1 \\
1 & k
\end{array}\right) \cdot t\right)
$$

depending on a complex parameter $\sigma$.

More generally, the Ruelle transfer operator of a map $T$ is defined as

$$
\left(L_{\sigma} f\right)(x, t)=\sum_{(y, s) \in T^{-1}(x, t)} \exp (h(y, s)) f(y, s),
$$

where we take $h(x, t)=-2 \sigma \log \left|T^{\prime}(x, t)\right|$. Clearly this operator is well suited for capturing the dynamical properties of the map $T$ as it is defined as a weighted sum over preimages. On the other hand, there is another operator that can be associated to a dynamical system and which typically has better spectral properties, but is less clearly related to the dynamics. The best circumstances are when these two agree (for a particular value of the parameter). The other operator is the PerronFrobenius operator $\mathcal{P}$. This is defined by the relation

$$
\int_{[0,1] \times \mathbb{P}} f(g \circ T) d \mu_{L e b}=\int_{[0,1] \times \mathbb{P}}(\mathcal{P} f) g d \mu_{L e b} .
$$

In the case of the shift $T$ of $(2.25)$ we have in fact that

$$
\mathcal{P}=\left.L_{\sigma}\right|_{\sigma=1}
$$

3.4.1. Spectral theory of $L_{1}$. In the case of the modular group $G=$ $\Gamma$, the spectral theory of the Perron-Frobenius operator of the Gauss shift was studied by D.Mayer [88]. More recently, Chang and Mayer [14] extended the results to the case of congruence subgroups. A similar approach is used in [83] to study the properties of the shift (2.25). 
The Perron-Frobenius operator

$$
\left(L_{1} f\right)(x, s)=\sum_{k=1}^{\infty} \frac{1}{(x+k)^{2}} f\left(\frac{1}{x+k},\left(\begin{array}{cc}
0 & 1 \\
1 & k
\end{array}\right) \cdot s\right)
$$

for the shift (2.25) has the following properties.

THEOREM 3.3. On a Banach space of holomorphic functions on $D \times \mathbb{P}$ continuous up to the boundary, with $D=\{z \in \mathbb{C}|| z-1 \mid<3 / 2\}$, under the condition (irreducibility)

$$
\mathbb{P}=\cup_{n=0}^{\infty}\left\{\left(\begin{array}{cc}
0 & 1 \\
1 & k_{1}
\end{array}\right) \cdots\left(\begin{array}{cc}
0 & 1 \\
1 & k_{n}
\end{array}\right)\left(t_{0}\right) \mid k_{1}, \ldots, k_{n} \geq 1\right\}
$$

the Perron-Frobenius operator $L_{1}$ has the following properties:

- $L_{1}$ is a nuclear operator, of trace class.

- $L_{1}$ has top eigenvalue $\lambda=1$. This eigenvalue is simple. The corresponding eigenfunction is (up to normalization)

$$
\frac{1}{(1+x)}
$$

- The rest of the spectrum of $L_{1}$ is contained in a ball of radius $r<1$.

- There is a complete set of eigenfunctions.

The irreducibility condition (2.34) is satisfied by congruence subgroups.

For other $T$-invariant subsets $E \subset[0,1] \times \mathbb{P}$, one can also consider operators $L_{E, \sigma}$ and $\mathcal{P}_{E}$. When the set has the property that $\mathcal{P}_{E}=$ $L_{E, \delta_{E}}$, for $\delta_{E}=\operatorname{dim}_{H} E$ the Hausdorff dimension, one can use the spectral theory of the operator $\mathcal{P}_{E}$ to study the dynamical properties of $T$.

The Lyapunov exponent can be read off the spectrum of the family of operators $L_{E, \sigma}$.

Lemma 3.4. Let $\lambda_{\sigma}$ denote the top eigenvalue of $L_{E, \sigma}$. Then

$$
\lambda(\beta)=\left.\frac{d}{d \sigma} \lambda_{\sigma}\right|_{\sigma=\operatorname{dim}_{H}(E)} \quad \mu_{H} \text { a.e. in } E
$$




\subsubsection{The Gauss problem. Let}

$$
m_{n}(x):=\text { measure of }\left\{\alpha \in(0,1) \mid x_{n}(\alpha) \leq x\right\}
$$

with $\alpha=\left[a_{1}(\alpha), \ldots, a_{n-1}(\alpha), a_{n}(\alpha)+x_{n}(\alpha)\right]$.

The asymptotic behavior of the measures $m_{n}$ is a famous problem on the distribution of continued fractions formulated by Gauss, who conjectured that

$$
m(x)=\lim _{n \rightarrow \infty} m_{n}(x) \stackrel{?}{=} \frac{1}{\log 2} \log (1+x) .
$$

The convergence of (2.35) to (2.36) was only proved by R. Kuzmin in 1928. Other proofs were then given by P. Lévy (1929), K. Babenko (1978) and D. Mayer (1991). The arguments used by Babenko and Mayer use the spectral theory on the Perron-Frobenius operator. Of these different arguments only the latter extends nicely to the case of the generalized Gauss shift (2.25).

The Gauss problem can be formulated in terms of a recursive relation

$$
m_{n+1}^{\prime}(x)=\left(L_{1} m_{n}^{\prime}\right)(x):=\sum_{k=1}^{\infty} \frac{1}{(x+k)^{2}} m_{n}^{\prime}\left(\frac{1}{x+k}\right) .
$$

The right hand side of (2.37) is the image of $m_{n}^{\prime}$ under the GaussKuzmin operator. This is nothing but the Perron-Frobenius operator for the shift $T$ in the case of the group $\Gamma=\operatorname{PGL}(2, \mathbb{Z})$.

As a consequence of Theorem 3.3, one obtains the following result.

THEOREM 3.5. Let

$$
m_{n}(x, t):=\mu_{L e b}\left\{(y, s): x_{n}(y) \leq x, g_{n}(y)^{-1}(s)=t\right\} .
$$

Then we have

- $m_{n}^{\prime}(x, t)=L_{1}^{n}(1)$.

- The limit $m(x, t)=\lim _{n \rightarrow \infty} m_{n}(x, t)$ exists and equals

$$
m(x, t)=\frac{1}{|\mathbb{P}| \log 2} \log (1+x) .
$$

This shows that there exists a unique $T$-invariant measure on $[0,1] \times$ $\mathbb{P}$. This is uniform in the discrete set $\mathbb{P}$ (the counting measure) and it is the Gauss measure of the shift of the continued fraction expansion on $[0,1]$. 


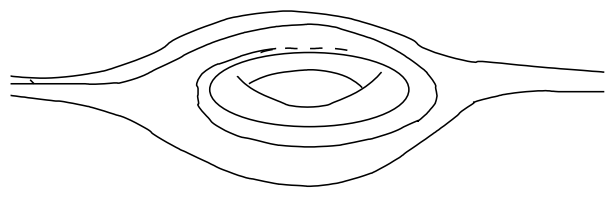

FiguRE 6. Limiting modular symbols: limiting cycle

3.5. Two theorems on limiting modular symbols. The result on the $T$-invariant measure allows us to study the general behavior of limiting modular symbols.

A special role is played by limiting modular symbols $\{\{*, \beta\}\}$ where $\beta$ is a quadratic irrationality in $\mathbb{R} \backslash \mathbb{Q}$.

THEOREM 3.6. Let $g \in G$ be hyperbolic, with eigenvalue $\Lambda_{g}$ corresponding to the attracting fixed point $\alpha_{g}^{+}$. Let $\Lambda(g):=\left|\log \Lambda_{g}\right|$, and let $\ell$ be the period of the continued fraction expansion of $\beta=\alpha_{g}^{+}$. Then

$$
\begin{gathered}
\{\{*, \beta\}\}_{G}=\frac{\{0, g(0)\}_{G}}{\Lambda(g)} \\
=\frac{1}{\lambda(\beta) \ell} \sum_{k=1}^{\ell}\left\{g_{k}^{-1}(\beta) \cdot 0, g_{k}^{-1}(\beta) \cdot i \infty\right\}_{G} .
\end{gathered}
$$

This shows that, in this case, the limiting modular symbols are linear combinations of classical modular symbols, with coefficients in the field generated over $\mathbb{Q}$ by the Lyapunov exponents $\lambda(\beta)$ of the quadratic irrationalities.

In terms of geodesics on the modular curve, this is the case where the geodesic has a limiting cycle given by the closed geodesic $\{0, g(0)\}_{G}$ (Figure 6).

There is then the "generic case", where, contrary to the previous example, the geodesics wind around many different cycles in such a way that the resulting homology class averages out to zero over long distances (Figure 7).

Theorem 3.7. For a $T$-invariant $E \subset[0,1] \times \mathbb{P}$, under the irreducibility condition for $E$,

$$
R_{\tau}(\beta, s):=\frac{1}{\tau}\{x, y(\tau)\}_{G}
$$

converges weakly to zero. Namely, for all $f \in L^{1}\left(E, d \mu_{H}\right)$,

$$
\lim _{\tau \rightarrow \infty} \int_{E} R_{\tau}(\beta, s) f(\beta, s) d \mu_{H}(\beta, s)=0 .
$$




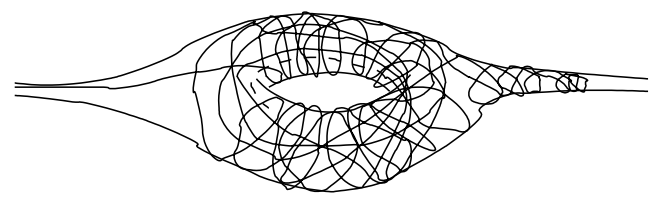

FIGURE 7. Limiting modular symbols: chaotic tangling and untangling

This weak convergence can be improved to strong convergence $\mu_{H}(E)$ almost everywhere. Thus, the limiting modular symbol satisfies

$$
\{\{*, \beta\}\}_{G} \equiv 0 \quad \text { a.e. on } E \text {. }
$$

This result depends upon the properties of the operator $L_{1}$ and the result on the $T$-invariant measure. In fact, to get the result on the weak convergence ([83]) one notices that the limit (2.30) computing limiting modular symbols can be evaluated in terms of a limit of iterates of the Perron-Frobenius operator, by

$$
\lim _{n} \frac{1}{\lambda_{0} n} \sum_{k=1}^{n} \int_{[0,1] \times \mathbb{P}} f\left(\varphi \circ T^{k}\right)=\lim _{n} \frac{1}{\lambda_{0} n} \sum_{k=1}^{n} \int_{[0,1] \times \mathbb{P}}\left(L_{1}^{k} f\right) \varphi,
$$

where $\lambda(\beta)=\lambda_{0}$ a.e. in $[0,1]$.

By the convergence of $L_{1}^{k} 1$ to the density $h$ of the $T$-invariant measure and of

$$
L_{1}^{k} f \rightarrow\left(\int f d \mu\right) h
$$

this yields

$$
\begin{gathered}
\int_{[0,1] \times \mathbb{P}}\{\{*, \beta\}\} f(\beta, t) d \mu(\beta, t)=\left(\int_{[0,1] \times \mathbb{P}} f d \mu\right)\left(\int_{[0,1] \times \mathbb{P}} \varphi h d \mu\right) \\
=\left(\int f d \mu\right) \frac{1}{2 \# \mathbb{P}} \sum_{s \in \mathbb{P}} \varphi(s) .
\end{gathered}
$$

It is then easy to check that the sum $\sum_{s \in \mathbb{P}} \varphi(s)=0$ since each term in the sum changes sign under the action of the inversion $\sigma \in \operatorname{PGL}(2, \mathbb{Z})$ with $\sigma^{2}=1$, but the sum is globally invariant under $\sigma$.

The argument extends to the case of other $T$-invariant subsets of $[0,1] \times \mathbb{P}$ for which the corresponding Perron-Frobenius operator $L_{E, \delta_{E}}$ has analogous properties $(c f .[84])$. The weak convergence proved by this type of argument can be improved to strong convergence by applying the strong law of large numbers to the "random variables" $\varphi_{k}=\varphi \circ T^{k}$ (cf. [84] for details). The result effectively plays the role of an ergodic theorem for the shift $T$ on $E$. 


\section{Hecke eigenforms}

A very important question is what happens to modular forms at the noncommutative boundary of the modular curves. There is a variety of phenomena in the theory of modular forms that hint to the fact that a suitable class of "modular forms" survives on the noncommutative boundary. Zagier introduced the term "quantum modular forms" to denote this important and yet not sufficiently understood class of examples. Some aspects of modular forms "pushed" to the noncommutative boundary were analyzed in [83], in the form of certain averages involving modular symbols and Dirichlet series related to modular forms of weight 2 . We recall here the main results in this case.

We shall now consider the case of congruence subgroups $G=\Gamma_{0}(p)$, for $p$ a prime.

Let $\omega$ be a holomorphic differential (also called a differential of the first kind) on the modular curve $X_{\Gamma_{0}(p)}=\Gamma_{0}(p) \backslash \mathbb{H}$. Let $\Phi=\varphi^{*}(\omega) / d z$ be the pullback under the projection $\varphi: \mathbb{H} \rightarrow X_{\Gamma_{0}(p)}$.

Let $\Phi$ be an eigenfunction for all the Hecke operators

$$
T_{n}=\sum_{d \mid n} \sum_{b=0}^{d-1}\left(\begin{array}{cc}
n / d & b \\
0 & d
\end{array}\right)
$$

with $(p, n)=1$,

$$
T_{n} \Phi=c_{n} \Phi .
$$

Then the L-function of $\omega$ is given by

$$
\begin{gathered}
L_{\omega}(s)=-\frac{(2 \pi)^{s}}{(2 \pi i) \Gamma(s)} \int_{0}^{\infty} \Phi(i y) y^{s-1} d y \\
L_{\omega}(s)=\sum_{n=1}^{\infty} c_{n} n^{-s} .
\end{gathered}
$$

There are many very interesting arithmetic properties of the integrals of such Hecke eigenforms on modular symbols (Manin [81]).

In particular, one has $([\mathbf{8 1}])$ the following relation between $L_{\omega}$ and modular symbols:

$$
\left(\sigma(n)-c_{n}\right) L_{\omega}(1)=\sum_{d \mid n, b \bmod d} \int_{\{0, b / d\}} \omega,
$$

with $\sigma(n)=\sum_{d \mid n} d$, from which one obtains

$$
\begin{aligned}
& \sum_{q:(q, p)=1} q^{-(2+t)} \sum_{q^{\prime} \leq q,\left(q, q^{\prime}\right)=1} \int_{\left\{0, q^{\prime} / q\right\}} \omega \\
& =\left[\frac{\zeta^{(p)}(1+t)}{\zeta^{(p)}(2+t)}-\frac{L_{\omega}^{(p)}(2+t)}{\zeta^{(p)}(2+t)^{2}}\right] \int_{0}^{i \infty} \phi^{*}(\omega)
\end{aligned}
$$


Here $L_{\omega}^{(p)}$ is the Mellin transform of $\Phi$ with omitted Euler $p$-factor, and $\zeta(s)$ the Riemann zeta, with corresponding $\zeta^{(p)}$, that is, $L_{\omega}^{(p)}(s)=$ $\sum_{n:(n, p)=1} c_{n} n^{-s}$ and $\zeta^{(p)}(s)=\sum_{n:(n, p)=1} n^{-s}$.

It is therefore interesting to study the properties of integrals on limiting modular symbols ( $c f$. [83]) and extensions of (2.38) (2.39).

To this purpose, in $[83]$ a suitable class of functions on the boundary $\mathbb{P}^{1}(\mathbb{R}) \times \mathbb{P}($ or just $[0,1] \times \mathbb{P})$ was introduced.

These are functions of the form

$$
\ell(f, \beta)=\sum_{k=1}^{\infty} f\left(q_{k}(\beta), q_{k-1}(\beta)\right) .
$$

Here $f$ is a complex valued function defined on pairs of coprime integers $\left(q, q^{\prime}\right)$ with $q \geq q^{\prime} \geq 1$ and with $f\left(q, q^{\prime}\right)=O\left(q^{-\epsilon}\right)$ for some $\epsilon>0$, and $q_{k}(\beta)$ are the successive denominators of the continued fraction expansion of $\beta \in[0,1]$.

The reason for this choice of functions is given by the following classical result of Lévy (1929).

Proposition 4.1. For a function $f$ as above, we have

$$
\int_{0}^{1} \ell(f, \alpha) d \alpha=\sum_{q \geq q^{\prime} \geq 1,\left(q, q^{\prime}\right)=1} \frac{f\left(q, q^{\prime}\right)}{q\left(q+q^{\prime}\right)},
$$

Sums and integrals here converge absolutely and uniformly.

We can interpret the summing over pairs of successive denominators as a property that replaces modularity, when "pushed to the boundary".

Through this class of functions it is possible to recast certain averages related to modular symbols on $X_{G}$, completely in terms of function theory on the "boundary" space $\Gamma \backslash\left(\mathbb{P}^{1}(\mathbb{R}) \times \mathbb{P}\right)$. A typical result of this sort is the following $([83])$.

Upon choosing

$$
f\left(q, q^{\prime}\right)=\frac{q+q^{\prime}}{q^{1+t}} \int_{\left\{0, q^{\prime} / q\right\}_{G}} \omega
$$

one obtains

$$
\begin{gathered}
\int_{0}^{1} \ell(f, \beta) d \mu_{L e b}(\beta)= \\
{\left[\frac{\zeta(1+t)}{\zeta(2+t)}-\frac{L_{\omega}^{(p)}(2+t)}{\zeta^{(p)}(2+t)^{2}}\right] \int_{0}^{i \infty} \phi^{*}(\omega),}
\end{gathered}
$$


which expresses the Mellin transform of a Hecke eigenform in terms of a boundary average.

Analogous results exist for different choices of $f\left(q, q^{\prime}\right)$, for Eisenstein series twisted by modular symbols, double logarithms at roots of unity, etc.

In fact, this result also shows that, even when the limiting modular symbol vanishes, one can still associate nontrivial homology classes to the geodesics with irrational ends.

In fact, for the case of $G=\Gamma_{0}(p)$ let us consider

$$
C(f, \beta):=\sum_{n=1}^{\infty} \frac{q_{n+1}(\beta)+q_{n}(\beta)}{q_{n+1}(\beta)^{1+t}}\left\{0, \frac{q_{n}(\beta)}{q_{n+1}(\beta)}\right\}_{\Gamma_{0}(p)} .
$$

This defines, for $\Re(t)>0$ and for almost all $\beta$, a homology class in $H_{1}\left(X_{G}\right.$, cusps, $\left.\mathbb{R}\right)$ such that the integral average

$$
\ell(f, \beta)=\int_{C(f, \beta)} \omega
$$

on $[0,1]$ recovers Mellin transforms of cusp forms by

$$
\int_{0}^{1} \int_{C(f, \beta)} \omega d \mu_{L e b}(\beta)=\left[\frac{\zeta(1+t)}{\zeta(2+t)}-\frac{L_{\omega}^{(p)}(2+t)}{\zeta^{(p)}(2+t)^{2}}\right] \int_{0}^{i \infty} \phi^{*}(\omega) .
$$

An estimate

$$
\frac{q_{n+1}(\beta)+q_{n}(\beta)}{q_{n+1}(\beta)^{1+t}}\left\{0, \frac{q_{n}(\beta)}{q_{n+1}(\beta)}\right\}_{G} \sim e^{-(5+2 t) n \lambda(\beta)} \sum_{k=1}^{n} \varphi \circ T^{k}(s)
$$

shows that the series involved are absolutely convergent.

\section{Selberg zeta function}

Infinite geodesics on $X_{G}$ are the images of infinite geodesics on $\mathbb{H}^{2} \times \mathbb{P}$ with ends on $\mathbb{P}^{1}(\mathbb{R}) \times \mathbb{P}$. Thus, they can be coded by $\left(\omega^{-}, \omega^{+}, s\right)$, with $\left(\omega^{ \pm}, s\right)=$ endpoints in $\mathbb{P}^{1}(\mathbb{R}) \times\{s\}, s \in \mathbb{P}, \omega^{-} \in(-\infty,-1]$, $\omega^{+} \in[0,1]$.

If $\omega^{ \pm}$are not cusp points, then we have infinite continued fraction expansions of these endpoints,

$$
\begin{aligned}
\omega^{+} & =\left[k_{0}, k_{1}, \ldots k_{r}, k_{r+1}, \ldots\right] \\
\omega^{-} & =\left[k_{-1} ; k_{-2}, \ldots, k_{-n}, k_{-n-1}, \ldots\right]
\end{aligned},
$$

hence the corresponding geodesics are coded by $(\omega, s), s \in \mathbb{P}$, where $\omega$ is a doubly infinite sequence

$$
\omega=\ldots k_{-(n+1)} k_{-n} \ldots k_{-1} k_{0} k_{1} \ldots k_{n} \ldots
$$


The equivalence relation of passing to the quotient by the group action is implemented by the invertible (double sided) shift:

$$
T\left(\omega^{+}, \omega^{-}, s\right)=\left(\frac{1}{\omega^{+}}-\left[\frac{1}{\omega^{+}}\right], \frac{1}{\omega^{-}-\left[1 / \omega^{+}\right]},\left(\begin{array}{cc}
-\left[1 / \omega^{+}\right] & 1 \\
1 & 0
\end{array}\right) \cdot s\right) .
$$

In particular, the closed geodesics in $X_{G}$ correspond to the case where the endpoints $\omega^{ \pm}$are the attractive and repelling fixed points of a hyperbolic element in the group. This corresponds to the case where $(\omega, s)$ is a periodic point for the shift $T$.

The Selberg zeta function is a suitable "generating function" for the closed geodesics in $X_{G}$. It is given by

$$
Z_{G}(s)=\prod_{m=0}^{\infty} \prod_{\gamma}\left(1-e^{-(s+m) \ell(\gamma)}\right),
$$

where $\gamma$ is a primitive closed geodesic of length $\ell(\gamma)$.

This can also be expressed in terms of the generalized Gauss shift by the following $([\mathbf{1 4}],[83])$

THEOREM 5.1. The Selberg zeta function for $G \subset \operatorname{PGL}(2, \mathbb{Z})$ of finite index is computed by the Fredholm determinant of the Ruelle transfer operator (2.32),

$$
Z_{G}(s)=\operatorname{det}\left(1-L_{s}\right) .
$$

An analogous result holds for finite index subgroups of $\operatorname{SL}(2, \mathbb{Z})$, where one gets $\operatorname{det}\left(1-L_{s}^{2}\right)$. There are also generalizations to other $T$ invariant sets $E$, where $\operatorname{det}\left(1-L_{E, s}\right)$ corresponds to a suitable "Selberg zeta" that only counts certain classes of closed geodesics.

\section{The modular complex and $K$-theory of $\mathrm{C}^{*}$-algebras}

When we interpret the "boundary" $\mathbb{P}^{1}(\mathbb{R}) \times \mathbb{P}$ with the action of $\Gamma$ as the noncommutative space $\mathrm{C}^{*}$-algebra $\mathrm{C}\left(\mathbb{P}^{1}(\mathbb{R}) \times \mathbb{P}\right) \rtimes \Gamma$, it is possible to reinterpret some of the arithmetic properties of modular curves in terms of these operator algebras. For instance, the modular symbols determine certain elements in the $K$-theory of this $\mathrm{C}^{*}$-algebra, and the modular complex and the exact sequence of relative homology (2.12) can be identified canonically with the Pimsner six terms exact sequence for $K$-theory of this $\mathrm{C}^{*}$-algebra.

For $\Gamma=\operatorname{PSL}(2, \mathbb{Z})$ and $G \subset \Gamma$ a finite index subgroup, we use the notation $X:=\mathbb{P}^{1}(\mathbb{R}) \times \mathbb{P}$, and $\Gamma_{\sigma}:=\langle\sigma\rangle=\mathbb{Z} / 2$ and $\Gamma_{\tau}:=\langle\tau\rangle=\mathbb{Z} / 3$.

The group $\Gamma$ acts on the tree with edges $T^{1} \simeq \Gamma$ and vertices $T^{0} \simeq \Gamma /\langle\sigma\rangle \cup \Gamma /\langle\tau\rangle$. This tree is embedded in $\mathbb{H}^{2}$ with vertices at 

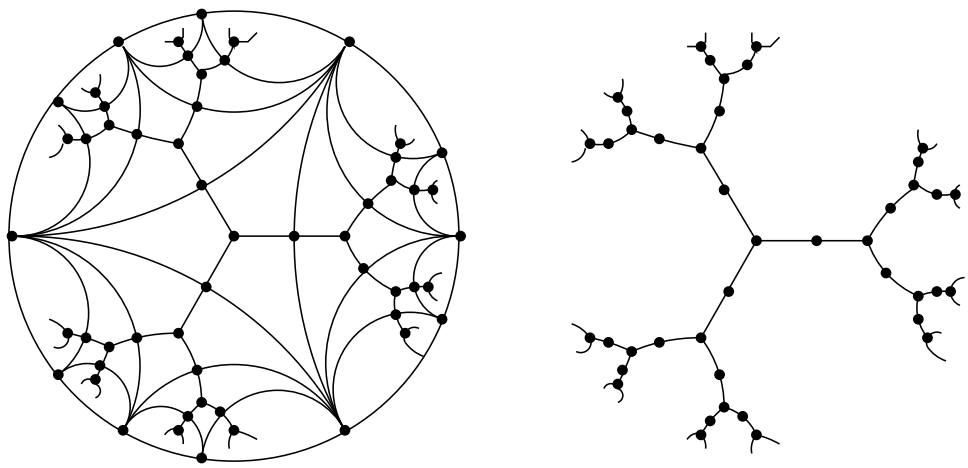

Figure 8. The modular complex and the tree of $\operatorname{PSL}(2, \mathbb{Z})$

the elliptic points and geodesic edges, as the dual graph of the triangulation (Figure 3) of the modular complex (Figure 8).

For a group acting on a tree there is a Pimsner six terms exact sequence, computing the $K$-theory of the crossed product $C^{*}$-algebra (for $\mathcal{A}=C(X)$ )

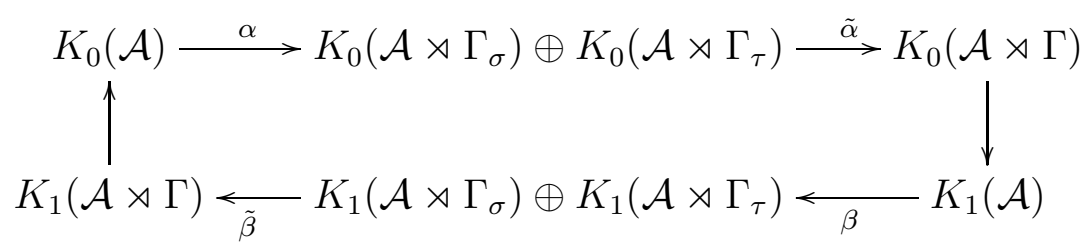

A direct inspection of the maps in this exact sequence shows that it contains a subsequence canonically isomorphic to the algebraic presentation of the modular complex, compatibly with the covering maps between modular curves for different congruence subgroups.

THEOREM 6.1. The algebraic presentation of the modular complex

$$
0 \rightarrow H_{\text {cusps }} \rightarrow \mathbb{Z}[\mathbb{P}] \rightarrow \mathbb{Z}\left[\mathbb{P}_{R}\right] \oplus \mathbb{Z}\left[\mathbb{P}_{I}\right] \rightarrow \mathbb{Z} \rightarrow 0
$$

is canonically isomorphic to the sequence

$$
0 \rightarrow \operatorname{Ker}(\beta) \rightarrow \mathbb{Z}[\mathbb{P}] \rightarrow \mathbb{Z}\left[\mathbb{P}_{I}\right] \oplus \mathbb{Z}\left[\mathbb{P}_{R}\right] \rightarrow \operatorname{Im}(\tilde{\beta}) \rightarrow 0
$$

A more geometric explanation for this exact sequence is the following. The Lie group $\operatorname{PSL}(2, \mathbb{R})$ can be identified with the circle bundle (unit sphere bundle) over the hyperbolic plane $\mathbb{H}=\operatorname{PSL}(2, \mathbb{R}) / P S O(2)$. Thus, the unit tangent bundle to the modular curve $X_{G}$ is given by the quotient $T_{1} X_{G}=G \backslash \operatorname{PSL}(2, \mathbb{R})$.

On the other hand, we can identify $\mathbb{P}^{1}(\mathbb{R})=B \backslash \operatorname{PSL}(2, \mathbb{R})$, the quotient by the upper triangular matrices $B$, so that we have Morita 
equivalent algebras

$$
C\left(\mathbb{P}^{1}(\mathbb{R})\right) \rtimes G \simeq C\left(T_{1} X_{G}\right) \rtimes B .
$$

The right hand side is the semidirect product of two $\mathbb{R}$-actions. Thus, by the Thom isomorphism [17] we have

$$
K_{*}\left(C\left(T_{1} X_{G}\right) \rtimes B\right) \cong K_{*}\left(C\left(T_{1} X_{G}\right)\right)
$$

where $K^{*}\left(T_{1} X_{G}\right)$ is related to $K^{*}\left(X_{G}\right)$ by the Gysin exact sequence.

A construction of crucial importance in noncommutative geometry is the classifying space for proper actions $\underline{B} G$ of Baum-Connes [7], the homotopy quotient $X \times_{G} \underline{E} G$ and the $\mu$-map

$$
\mu: K^{*}\left(X \times_{G} \underline{E} G\right) \rightarrow K_{*}(C(X) \rtimes G)
$$

relating the topologically defined $K$-theory to the analytic $K$-theory of $C^{*}$-algebras.

In a sense, for noncommutative spaces that are obtained as quotients by "bad" equivalence relations, the homotopy quotient is a "commutative shadow" from which much crucial information on the topology can be read ( $c f .[\mathbf{7}],[\mathbf{1 9}])$.

In our case we have $\underline{E} G=\mathbb{H}^{2}$ and the $\mu$-map is an isomorphism (the Baum-Connes conjecture holds). By retracting $\underline{E} G=\mathbb{H}^{2}$ to the tree of $\operatorname{PSL}(2, \mathbb{Z})$ and applying the Mayer-Vietoris sequence to vertices and edges one obtains the Pimsner six terms exact sequence.

\section{Intermezzo: Chaotic Cosmology}

We digress momentarily on a topic from General Relativity, which turns out to be closely related to the "noncommutative compactification" of the modular curve $X_{G}$ with $G$ the congruence subgroup $\Gamma_{0}(2)$ $([83])$.

An important problem in cosmology is understanding how anisotropy in the early universe affects the long time evolution of space-time. This problem is relevant to the study of the beginning of galaxy formation and in relating the anisotropy of the background radiation to the appearance of the universe today.

We follow [6] for a brief summary of anisotropic and chaotic cosmology. The simplest significant cosmological model that presents strong anisotropic properties is given by the Kasner metric

$$
d s^{2}=-d t^{2}+t^{2 p_{1}} d x^{2}+t^{2 p_{2}} d y^{2}+t^{2 p_{3}} d z^{2},
$$

where the exponents $p_{i}$ are constants satisfying $\sum p_{i}=1=\sum_{i} p_{i}^{2}$. Notice that, for $p_{i}=d \log g_{i i} / d \log g$, the first constraint $\sum_{i} p_{i}=1$ is just the condition that $\log g_{i j}=2 \alpha \delta_{i j}+\beta_{i j}$ for a traceless $\beta$, while 
the second constraint $\sum_{i} p_{i}^{2}=1$ amounts to the condition that, in the Einstein equations written in terms of $\alpha$ and $\beta_{i j}$,

$$
\begin{gathered}
\left(\frac{d \alpha}{d t}\right)^{2}=\frac{8 \pi}{3}\left(T^{00}+\frac{1}{16 \pi}\left(\frac{d \beta_{i j}}{d t}\right)^{2}\right) \\
e^{-3 \alpha} \frac{d}{d t}\left(e^{3 \alpha} \frac{d \beta_{i j}}{d t}\right)=8 \pi\left(T_{i j}-\frac{1}{3} \delta_{i j} T_{k k}\right),
\end{gathered}
$$

the term $T^{00}$ is negligible with respect to the term $\left(d \beta_{i j} / d t\right)^{2} / 16 \pi$, which is the "effective energy density" of the anisotropic motion of empty space, contributing together with a matter term to the Hubble constant.

Around 1970, Belinskii, Khalatnikov, and Lifshitz introduced a cosmological model (mixmaster universe) where they allowed the exponents $p_{i}$ of the Kasner metric to depend on a parameter $u$,

$$
\begin{aligned}
& p_{1}=\frac{-u}{1+u+u^{2}} \\
& p_{2}=\frac{1+u}{1+u+u^{2}} \\
& p_{3}=\frac{u(1+u)}{1+u+u^{2}}
\end{aligned}
$$

Since for fixed $u$ the model is given by a Kasner space-time, the behavior of this universe can be approximated for certain large intervals of time by a Kasner metric. In fact, the evolution is divided into Kasner eras and each era into cycles. During each era the mixmaster universe goes through a volume compression. Instead of resulting in a collapse, as with the Kasner metric, high negative curvature develops resulting in a bounce (transition to a new era) which starts again a behavior approximated by a Kasner metric, but with a different value of the parameter $u$. Within each era, most of the volume compression is due to the scale factors along one of the space axes, while the other scale factors alternate between phases of contraction and expansion. These alternating phases separate cycles within each era.

More precisely, we are considering metrics generalizing the Kasner metric (2.43), which still admit $S O(3)$ symmetry on the space-like hypersurfaces and present a singularity at $t \rightarrow 0$. In terms of logarithmic time $d \Omega=-\frac{d t}{a b c}$, the mixmaster universe model of Belinskii, Khalatnikov, and Lifshitz admits a discretization with the following properties:

1. The time evolution is divided into Kasner eras $\left[\Omega_{n}, \Omega_{n+1}\right]$, for $n \in \mathbb{Z}$. At the beginning of each era we have a corresponding discrete value of the parameter $u_{n}>1$ in $(2.44)$. 
2. Each era, where the parameter $u$ decreases with growing $\Omega$, can be subdivided in cycles corresponding to the discrete steps $u_{n}, u_{n}-1$, $u_{n}-2$, etc. A change $u \rightarrow u-1$ corresponds, after acting with the permutation $(12)(3)$ on the space coordinates, to changing $u$ to $-u$, hence replacing contraction with expansion and conversely. Within each cycle the space-time metric is approximated by the Kasner metric (2.43) with the exponents $p_{i}$ in (2.44) with a fixed value of $u=u_{n}-k>$ 1.

3. An era ends when, after a number of cycles, the parameter $u_{n}$ falls in the range $0<u_{n}<1$. Then the bouncing is given by the transition $u \rightarrow 1 / u$ which starts a new series of cycles with new Kasner parameters and a permutation (1)(23) of the space axis, in order to have again $p_{1}<p_{2}<p_{3}$ as in $(2.44)$.

Thus, the transition formula relating the values $u_{n}$ and $u_{n+1}$ of two successive Kasner eras is

$$
u_{n+1}=\frac{1}{u_{n}-\left[u_{n}\right]},
$$

which is exactly the shift of the continued fraction expansion, $T x=$ $1 / x-[1 / x]$, with $x_{n+1}=T x_{n}$ and $u_{n}=1 / x_{n}$.

The previous observation is the key to a geometric description of solutions of the mixmaster universe in terms of geodesics on a modular curve $(c f .[83])$.

THEOREM 7.1. Consider the modular curve $X_{\Gamma_{0}(2)}$. Each infinite geodesic $\gamma$ on $X_{\Gamma_{0}(2)}$ not ending at cusps determines a mixmaster universe.

In fact, an infinite geodesic on $X_{\Gamma_{0}(2)}$ is the image under the quotient map

$$
\pi_{\Gamma}: \mathbb{H}^{2} \times \mathbb{P} \rightarrow \Gamma \backslash\left(\mathbb{H}^{2} \times \mathbb{P}\right) \cong X_{G},
$$

where $\Gamma=\operatorname{PGL}(2, \mathbb{Z}), G=\Gamma_{0}(2)$, and $\mathbb{P}=\Gamma / G \cong \mathbb{P}^{1}\left(\mathbb{F}_{2}\right)=\{0,1, \infty\}$, of an infinite geodesic on $\mathbb{H}^{2} \times \mathbb{P}$ with ends on $\mathbb{P}^{1}(\mathbb{R}) \times \mathbb{P}$. We consider the elements of $\mathbb{P}^{1}\left(\mathbb{F}_{2}\right)$ as labels assigned to the three space axes, according to the identification

$$
\begin{array}{lll}
0=[0: 1] & \mapsto & z \\
\infty=[1: 0] & \mapsto & y \\
1=[1: 1] & \mapsto & x .
\end{array}
$$

As we have seen, geodesics can be coded in terms of data $\left(\omega^{-}, \omega^{+}, s\right)$ with the action of the shift $T$.

The data $(\omega, s)$ determine a mixmaster universe, with the $k_{n}=$ $\left[u_{n}\right]=\left[1 / x_{n}\right]$ in the Kasner eras, and with the transition between 
subsequent Kasner eras given by $x_{n+1}=T x_{n} \in[0,1]$ and by the permutation of axes induced by the transformation

$$
\left(\begin{array}{cc}
-k_{n} & 1 \\
1 & 0
\end{array}\right)
$$

acting on $\mathbb{P}^{1}\left(\mathbb{F}_{2}\right)$. It is easy to verify that, in fact, this acts as the permutation $0 \mapsto \infty, 1 \mapsto 1, \infty \mapsto 0$, if $k_{n}$ is even, and $0 \mapsto \infty$, $1 \mapsto 0, \infty \mapsto 1$ if $k_{n}$ is odd, that is, after the identification (2.45), as the permutation (1)(23) of the space axes $(x, y, z)$, if $k_{n}$ is even, or as the product of the permutations $(12)(3)$ and (1)(23) if $k_{n}$ is odd. This is precisely what is obtained in the mixmaster universe model by the repeated series of cycles within a Kasner era followed by the transition to the next era.

Data $(\omega, s)$ and $T^{m}(\omega, s), m \in \mathbb{Z}$, determine the same solution up to a different choice of the initial time.

There is an additional time-symmetry in this model of the evolution of mixmaster universes $(c f$. [6]). In fact, there is an additional parameter $\delta_{n}$ in the system, which measures the initial amplitude of each cycle. It is shown in [6] that this is governed by the evolution of a parameter

$$
v_{n}=\frac{\delta_{n+1}\left(1+u_{n}\right)}{1-\delta_{n+1}}
$$

which is subject to the transformation across cycles $v_{n+1}=\left[u_{n}\right]+v_{n}^{-1}$. By setting $y_{n}=v_{n}^{-1}$ we obtain

$$
y_{n+1}=\frac{1}{\left(y_{n}+\left[1 / x_{n}\right]\right)},
$$

hence we see that we can interpret the evolution determined by the data $\left(\omega^{+}, \omega^{-}, s\right)$ with the shift $T$ either as giving the complete evolution of the $u$-parameter towards and away from the cosmological singularity, or as giving the simultaneous evolution of the two parameters $(u, v)$ while approaching the cosmological singularity.

This in turn determines the complete evolution of the parameters $(u, \delta, \Omega)$, where $\Omega_{n}$ is the starting time of each cycle. For the explicit recursion $\Omega_{n+1}=\Omega_{n+1}\left(\Omega_{n}, x_{n}, y_{n}\right)$ see $[6]$.

The result of Theorem 3.5 on the unique $T$-invariant measure on $[0,1] \times \mathbb{P}$

$$
d \mu(x, s)=\frac{\delta(s) d x}{3 \log (2)(1+x)},
$$


implies that the alternation of the space axes is uniform over the time evolution, namely the three axes provide the scale factor responsible for volume compression with equal frequencies.

The Perron-Frobenius operator for the shift (2.25) yields the density of the invariant measure (2.46) satisfying $L_{1} f=f$. The top eigenvalue $\eta_{\sigma}$ of $L_{\sigma}$ is related to the topological pressure by $\eta_{\sigma}=\exp (P(\sigma))$. This can be estimated numerically, using the technique of [5] and the integral kernel operator representation of $\S 1.3$ of [83].

The interpretation of solutions in terms of geodesics provides a natural way to single out and study certain special classes of solutions on the basis of their geometric properties. Physically, such special classes of solutions exhibit different behaviors approaching the cosmological singularity.

For instance, the data $\left(\omega^{+}, s\right)$ corresponding to an eventually periodic sequence $k_{0} k_{1} \ldots k_{m} \ldots$ of some period $\overline{a_{0} \ldots a_{\ell}}$ correspond to those geodesics on $X_{\Gamma_{0}(2)}$ that asymptotically wind around the closed geodesic identified with the doubly infinite sequence $\ldots a_{0} \ldots a_{\ell} a_{0} \ldots a_{\ell} \ldots$. Physically, these universes exhibit a pattern of cycles that recurs periodically after a finite number of Kasner eras.

Another special class of solutions is given by the Hensley Cantor sets $(c f$. [85]). These are the mixmaster universes for which there is a fixed upper bound $N$ to the number of cycles in each Kasner era.

In terms of the continued fraction description, these solutions correspond to data $\left(\omega^{+}, s\right)$ with $\omega^{+}$in the Hensley Cantor set $E_{N} \subset[0,1]$. The set $E_{N}$ is given by all points in $[0,1]$ with all digits in the continued fraction expansion bounded by $N(c f$. [62]). In more geometric terms, these correspond to geodesics on the modular curve $X_{\Gamma_{0}(2)}$ that wander only a finite distance into a cusp.

On the set $E_{N}$ the Ruelle and Perron-Frobenius operators are given by

$$
\left(L_{\sigma, N} f\right)(x, s)=\sum_{k=1}^{N} \frac{1}{(x+k)^{2 \sigma}} f\left(\frac{1}{x+k},\left(\begin{array}{cc}
0 & 1 \\
1 & k
\end{array}\right) \cdot s\right) .
$$

This operator still has a unique invariant measure $\mu_{N}$, whose density satisfies $\mathcal{L}_{2 \operatorname{dim}_{H}\left(E_{N}\right), N} f=f$, with

$$
\operatorname{dim}_{H}\left(E_{N}\right)=1-\frac{6}{\pi^{2} N}-\frac{72 \log N}{\pi^{4} N^{2}}+O\left(1 / N^{2}\right)
$$

the Hausdorff dimension of the Cantor set $E_{N}$. Moreover, the top eigenvalue $\eta_{\sigma}$ of $\mathcal{L}_{\sigma, N}$ is related to the Lyapunov exponent by

$$
\lambda(x)=\left.\frac{d}{d \sigma} \eta_{\sigma}\right|_{\sigma=\operatorname{dim}_{H}\left(E_{N}\right)},
$$


for $\mu_{N}$-almost all $x \in E_{N}$.

A consequence of the characterization of the time evolution in terms of the dynamical system (2.25) is that we can study global properties of suitable moduli spaces of mixmaster universes. For instance, the moduli space for time evolutions of the $u$-parameter approaching the cosmological singularity as $\Omega \rightarrow \infty$ is given by the quotient of $[0,1] \times \mathbb{P}$ by the action of the shift $T$.

Similarly, when we restrict to special classes of solutions, e.g. we can consider the "moduli space" $E_{N} \times \mathbb{P}$ modulo the action of $T$. In this example, the dynamical system $T$ acting on $E_{N} \times \mathbb{P}$ is a subshift of finite type, and the resulting noncommutative space is a Cuntz-Krieger algebra [51]. This is an interesting class of $C^{*}$-algebras, generated by partial isometries. Another such algebra will play a fundamental role in the geometry at arithmetic infinity, which is the topic of the next lecture.

In the example of the mixmaster dynamics on the Hensley Cantor sets, the shift $T$ is described by the Markov partition

$$
\mathcal{A}_{N}=\left\{((k, t),(\ell, s)) \mid U_{k, t} \subset T\left(U_{\ell, s}\right)\right\},
$$

for $k, \ell \in\{1, \ldots, N\}$, and $s, t \in \mathbb{P}$, with sets $U_{k, t}=U_{k} \times\{t\}$, where $U_{k} \subset E_{N}$ are the clopen subsets where the local inverses of $T$ are defined,

$$
U_{k}=\left[\frac{1}{k+1}, \frac{1}{k}\right] \cap E_{N} .
$$

This Markov partition determines a matrix $A_{N}$, with entries $\left(A_{N}\right)_{k t, \ell s}=$ 1 if $U_{k, t} \subset T\left(U_{\ell, s}\right)$ and zero otherwise ( $c f$. Figure 9$)$.

Proposition 7.2. The $3 \times 3$ submatrices $A_{k \ell}=\left(A_{(k, t),(\ell, s)}\right)_{s, t \in \mathbb{P}}$ of the matrix $A_{N}$ are of the form

$$
A_{k \ell}=\left\{\begin{array}{l}
\left(\begin{array}{lll}
0 & 0 & 1 \\
0 & 1 & 0 \\
1 & 0 & 0
\end{array}\right) \quad \ell=2 m \\
\left(\begin{array}{lll}
0 & 0 & 1 \\
1 & 0 & 0 \\
0 & 1 & 0
\end{array}\right) \quad \ell=2 m+1
\end{array}\right.
$$

This is just the condition $U_{k, t} \subset T\left(U_{\ell, s}\right)$ written as

$$
\left(\begin{array}{ll}
0 & 1 \\
1 & \ell
\end{array}\right) \cdot s=t
$$




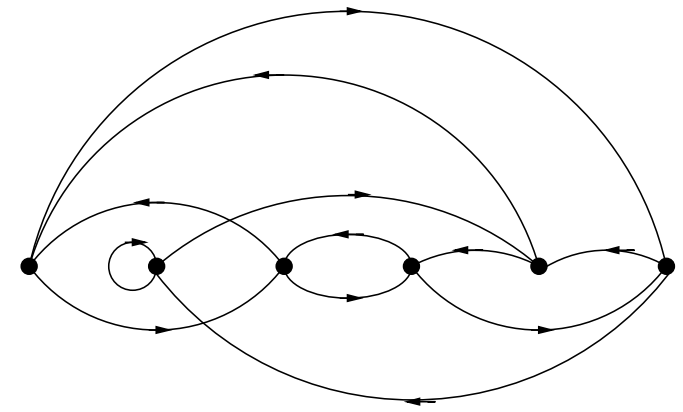

FiguRE 9. The directed graph of the matrix $A_{2}$

together with the fact that the transformation

$$
\left(\begin{array}{ll}
0 & 1 \\
1 & \ell
\end{array}\right)
$$

acts on $\mathbb{P}^{1}\left(\mathbb{F}_{2}\right)$ as the permutation $0 \mapsto \infty, 1 \mapsto 1, \infty \mapsto 0$, when $\ell$ is even, and $\infty \mapsto 0,0 \mapsto 1,1 \mapsto \infty$ if $\ell$ is odd.

As a noncommutative space associated to the Markov partition we consider the Cuntz-Krieger $\mathrm{C}^{*}$-algebra $\mathcal{O}_{A_{N}}$ (cf. [51]), which is the universal $\mathrm{C}^{*}$-algebra generated by partial isometries $S_{k t}$ satisfying the relations

$$
\begin{gathered}
\sum_{(k, t)} S_{k t} S_{k t}^{*}=1, \\
S_{\ell s}^{*} S_{\ell s}=\sum_{(k, t)} A_{(k, t),(\ell, s)} S_{k t} S_{k t}^{*} .
\end{gathered}
$$

Topological invariants of this $\mathrm{C}^{*}$-algebra reflect dynamical properties of the shift $T$. 


\section{CHAPTER 3}

\section{Quantum statistical mechanics and Galois theory}

This chapter is dedicated to a brief review of the joint work of Alain Connes and the author on quantum statistical mechanics of $\mathbb{Q}$-lattices $[32],[33]$, and to the results of the work of Alain Connes, Niranjan Ramachandran, and the author [38] on the construction of a quantum statistical mechanical system that fully recovers the explicit class field theory for imaginary quadratic fields in a way similar to what the BostConnes system does for the explicit class field theory of $\mathbb{Q}$. At the end of the chapter, we include a brief review of some of Manin's idea on real quadratic fields and noncommutative tori with real multiplication [76].

The main notion that we consider in this chapter is that of commensurability classes of $\mathbb{K}$-lattices, where $\mathbb{K}$ will be either $\mathbb{Q}$ or an imaginary quadratic field $\mathbb{Q}(\sqrt{-d})$, for $d$ some positive integer. We begin with the case of $\mathbb{Q}$, according to the following definition.

Definition 0.1. A $\mathbb{Q}$-lattice in $\mathbb{R}^{n}$ consists of a pair $(\Lambda, \phi)$ of a lattice $\Lambda \subset \mathbb{R}^{n}$ (a cocompact free abelian subgroup of $\mathbb{R}^{n}$ of rank $n$ ) together with a system of labels of its torsion points given by a homomorphism of abelian groups

$$
\phi: \mathbb{Q}^{n} / \mathbb{Z}^{n} \longrightarrow \mathbb{Q} \Lambda / \Lambda \text {. }
$$

$A \mathbb{Q}$-lattice is invertible if $\phi$ is an isomorphism.

Two $\mathbb{Q}$-lattices are commensurable,

$$
\left(\Lambda_{1}, \phi_{1}\right) \sim\left(\Lambda_{2}, \phi_{2}\right)
$$

iff $\mathbb{Q} \Lambda_{1}=\mathbb{Q} \Lambda_{2}$ and

$$
\phi_{1}=\phi_{2} \bmod \Lambda_{1}+\Lambda_{2}
$$

One can check that, indeed, commensurability defines an equivalence relation among $\mathbb{Q}$-lattices. The interesting aspect of this equivalence relation is that the quotient provides another typical case that is best described through noncommutative geometry. For this it is crucial that we consider non-invertible $\mathbb{Q}$-lattices. In fact, most $\mathbb{Q}$-lattices are not commensurable to an invertible one, while two invertible $\mathbb{Q}$-lattices are commensurable if and only if they are equal. 


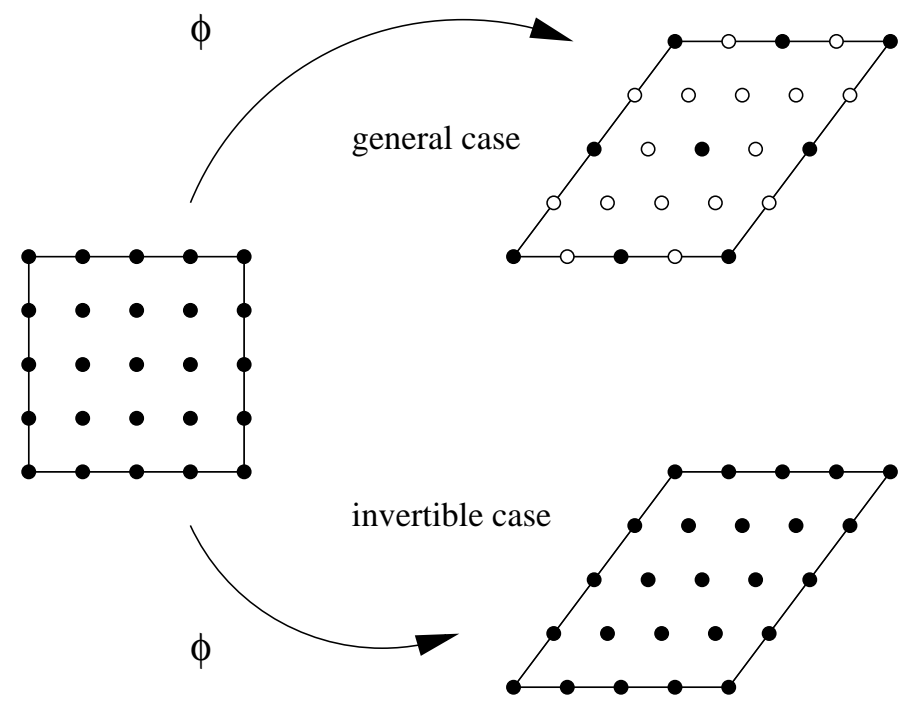

Figure 1. Generic and invertible 2-dimensional $\mathbb{Q}$ lattices

In the following, we denote by $\mathcal{L}_{n}$ the set of commensurability classes of $\mathbb{Q}$-lattices in $\mathbb{R}^{n}$. The topology on this space is encoded in a noncommutative algebra of coordinates, namely a $C^{*}$-algebra $C^{*}\left(\mathcal{L}_{n}\right)$. We will discuss in some detail only the cases $n=1$ (Bost-Connes system) and $n=2$ (the case considered in [32]). In these cases, we will look at commensurability classes of $\mathbb{Q}$-lattices up to scaling, namely the quotients $\mathcal{L}_{1} / \mathbb{R}_{+}^{*}$ and $\mathcal{L}_{2} / \mathbb{C}^{*}$. The corresponding $C^{*}$-algebras

$$
C^{*}\left(\mathcal{L}_{1} / \mathbb{R}_{+}^{*}\right) \quad \text { and } \quad C^{*}\left(\mathcal{L}_{2} / \mathbb{C}^{*}\right)
$$

are endowed with a natural time evolution (a 1-parameter family of automorphisms). Thus, one can consider them as quantum statistical mechanical systems and look for equilibrium states, depending on a thermodynamic parameter $\beta$ (inverse temperature). The interesting connection to arithmetic arises from the fact that the action of symmetries of the system on equilibrium states at zero temperature can be described in terms of Galois theory. In the 1-dimensional case of Bost-Connes, this will happen via the class field theory of $\mathbb{Q}$, namely the Galois theory of the cyclotomic field $\mathbb{Q}^{c y c l}$. In the two dimensional case, the picture is more elaborate and involves the automorphisms of the field of modular functions. 


\section{Quantum Statistical Mechanics}

In classical statistical mechanics a state is a probability measure $\mu$ on the phase space that assigns to each observable $f$ an expectation value, in the form of an average

$$
\int f d \mu
$$

In particular, for a Hamiltonian system, the macroscopic thermodynamic behavior is described via the Gibbs canonical ensemble. This is the measure

$$
d \mu_{G}=\frac{1}{Z} e^{-\beta H} d \mu_{\text {Liouville }},
$$

normalized by $Z=\int e^{-\beta H} d \mu_{\text {Liouville }}$, with a thermodynamic parameter $\beta=(k T)^{-1}$, for $T$ the temperature and $k$ the Boltzmann constant (which we can set equal to one in suitable units).

A quantum statistical mechanical system consists of the data of an algebra of observables (a $C^{*}$-algebra $\mathcal{A}$ ), together with a time evolution given as a 1-parameter family of automorphisms $\sigma_{t} \in \operatorname{Aut}(\mathcal{A})$. We refer to the pair $\left(\mathcal{A}, \sigma_{t}\right)$ as a $C^{*}$-dynamical system. These data describe the microscopic quantum mechanical evolution of the system.

The macroscopic thermodynamical properties are encoded in the equilibrium states of the system, depending on the inverse temperature $\beta$. While the Gibbs measure in the classical case is defined in terms of the Hamiltonian and the symplectic structure on the phase space, the notion of equilibrium state in the quantum statistical mechanical setting only depends on the algebra of observables and its time evolution, and does not involve any additional structure like the symplectic structure or the approximation by regions of finite volume.

We first need to recall the notion of states. These can be thought of as probability measures on noncommutative spaces.

Definition 1.1. Given a unital $C^{*}$-algebra $\mathcal{A}$, a state on $\mathcal{A}$ is a linear functional $\varphi: \mathcal{A} \rightarrow \mathbb{C}$ satisfying normalization and positivity,

$$
\varphi(1)=1, \quad \varphi\left(a^{*} a\right) \geq 0 .
$$

When the $C^{*}$-algebra $\mathcal{A}$ is non-unital, the condition $\varphi(1)=1$ is replaced by $\|\varphi\|=1$ where

$$
\|\varphi\|:=\sup _{x \in \mathcal{A},\|x\| \leq 1}|\varphi(x)| .
$$

Such states are restrictions of states on the unital $C^{*}$-algebra $\tilde{\mathcal{A}}$ obtained by adjoining a unit. 
Before giving the general definition of equilibrium states via the KMS condition, we can see equilibrium states in the simple case of a system with finitely many quantum degrees of freedom. In this case, the algebra of observables is the algebra of operators in a Hilbert space $\mathcal{H}$ and the time evolution is given by $\sigma_{t}(a)=e^{i t H} a e^{-i t H}$, where $H$ is a positive self-adjoint operator such that $\exp (-\beta H)$ is trace class for any $\beta>0$. For such a system, the analog of (3.2) is

$$
\varphi(a)=\frac{1}{Z} \operatorname{Tr}\left(a e^{-\beta H}\right) \quad \forall a \in A,
$$

with the normalization factor $Z=\operatorname{Tr}(\exp (-\beta H))$.

The Kubo-Martin-Schwinger condition (KMS) ( cf. [12], [58], [59]) describing equilibrium states of more general quantum statistical mechanical systems generalizes (3.5) beyond the range of temperatures where $\exp (-\beta H)$ is trace class.

Definition 1.2. Given a $C^{*}$-dynamical system $\left(\mathcal{A}, \sigma_{t}\right)$, a state $\varphi$ on $\mathcal{A}$ satisfies the KMS condition at inverse temperature $0<\beta<\infty$ iff, for all $a, b \in \mathcal{A}$, there exists a function $F_{a, b}(z)$ holomorphic on the strip $0<\Im(z)<\beta$ continuous to the boundary and bounded, such that for all $t \in \mathbb{R}$

$$
F_{a, b}(t)=\varphi\left(a \sigma_{t}(b)\right) \quad \text { and } \quad F_{a, b}(t+i \beta)=\varphi\left(\sigma_{t}(b) a\right) .
$$

$K M S_{\infty}$ states are weak limits of $K M S_{\beta}$ states as $\beta \rightarrow \infty$,

$$
\varphi_{\infty}(a)=\lim _{\beta \rightarrow \infty} \varphi_{\beta}(a) \quad \forall a \in \mathcal{A} .
$$

Our definition of $\mathrm{KMS}_{\infty}$ state is stronger than the one often adopted in the literature, which simply uses the existence, for each $a, b \in \mathcal{A}$, of a bounded holomorphic function $F_{a, b}(z)$ on the upper half plane such that $F_{a, b}(t)=\varphi\left(a \sigma_{t}(b)\right)$. It is easy to see that this notion, which we simply call the ground states of the system, is in fact weaker than the notion of $\mathrm{KMS}_{\infty}$ states given in Definition 1.2. For example, in the simplest case of the trivial time evolution, all states are ground states, while only tracial states are $\mathrm{KMS}_{\infty}$ states. Another advantage of our definition is that, for all $0<\beta \leq \infty$, the $\mathrm{KMS}_{\beta}$ states form a weakly compact convex set, hence we can consider the set $\mathcal{E}_{\beta}$ of its extremal points. These provide a good notion of points for the underlying noncommutative space. This will be especially useful in connection to an arithmetic structure specified by an arithmetic subalgebra $\mathcal{A}_{\mathbb{Q}}$ of $\mathcal{A}$ on which one evaluates the $\mathrm{KMS}_{\infty}$ states. This will play a key role in the relation between the symmetries of the system and the action of the Galois group on states $\varphi \in \mathcal{E}_{\infty}$ evaluated on $\mathcal{A}_{\mathbb{Q}}$. 


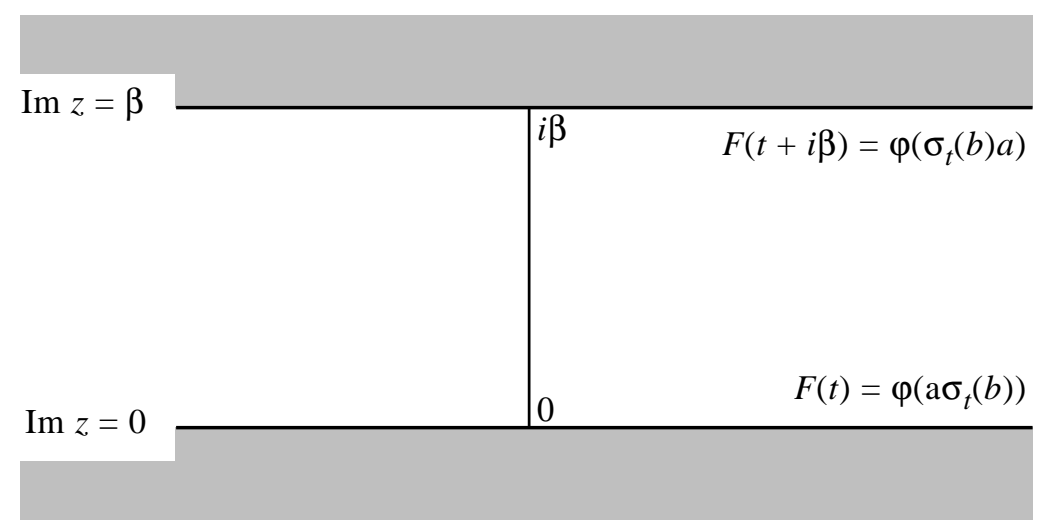

FiguRE 2. The KMS condition

1.1. Symmetries. An important role in quantum statistical mechanics is played by symmetries. Typically, symmetries of the algebra $\mathcal{A}$ compatible with the time evolution induce symmetries of the equilibrium states $\mathcal{E}_{\beta}$ at different temperatures. Especially important are the phenomena of symmetry breaking. In such cases, there is a global underlying group $G$ of symmetries of the algebra $\mathcal{A}$, but in certain ranges of temperature the choice of an equilibrium state $\varphi$ breaks the symmetry to a smaller subgroup $G_{\varphi}=\left\{g \in G: g^{*} \varphi=\varphi\right\}$, where $g^{*}$ denotes the induced action on states. Various systems can exhibit one or more phase transitions, or none at all. A typical situation in physical systems sees a unique KMS state for all values of the parameter above a certain critical temperature $\left(\beta<\beta_{c}\right)$. This corresponds to a chaotic phase such as randomly distributed spins in a ferromagnet. When the system cools down and reaches the critical temperature, the unique equilibrium state branches off into a larger set $\mathrm{KMS}_{\beta}$ and the symmetry is broken by the choice of an extremal state in $\mathcal{E}_{\beta}$.

We will see that the case of $\mathcal{L}_{1} / \mathbb{R}_{+}^{*}$ gives rise to a system with a single phase transition $([\mathbf{9}])$, while in the case of $\mathcal{L}_{2} / \mathbb{C}^{*}$ the system has multiple phase transitions.

A very important point is that we need to consider both symmetries by automorphisms and by endomorphisms.

Automorphisms: A subgroup $G \subset \operatorname{Aut}(\mathcal{A})$ is compatible with $\sigma_{t}$ if for all $g \in G$ and for all $t \in \mathbb{R}$ we have $g \sigma_{t}=\sigma_{t} g$. There is then an 
induced action of $G$ on KMS states and in particular on the set $\mathcal{E}_{\beta}$. If $u$ is a unitary, acting on $\mathcal{A}$ by

$$
\text { Ad } u: \quad a \mapsto u a u^{*}
$$

and satisfying $\sigma_{t}(u)=u$, then we say that $\operatorname{Ad} u$ is an inner automorphism of $\left(\mathcal{A}, \sigma_{t}\right)$. Inner automorphisms act trivially on KMS states.

Endomorphisms: Let $\rho$ be a $*$-homomorphism satisfying $\rho \sigma_{t}=\sigma_{t} \rho$. Consider the idempotent $e=\rho(1)$. If $\varphi \in \mathcal{E}_{\beta}$ is a state such that $\varphi(e) \neq 0$, then there is a well defined pullback $\rho^{*} \varphi$,

$$
\rho^{*}(\varphi)=\frac{1}{\varphi(e)} \varphi \circ \rho .
$$

Let $u$ be an isometry compatible with the time evolution by

$$
\sigma_{t}(u)=\lambda^{i t} u \quad \lambda>0 .
$$

One has $u^{*} u=1$ and $u u^{*}=e$. We say that Ad $u$ defined by $a \mapsto u a u^{*}$ is an inner endomorphism of $\left(\mathcal{A}, \sigma_{t}\right)$. The condition (3.9) ensures that $(\operatorname{Ad} u)^{*} \varphi$ is well defined according to (3.8) and the KMS condition shows that the induced action of an inner endomorphism on KMS states is trivial.

One needs to be especially careful in defining the action of endomorphisms by (3.8). In fact, there are cases where for $\mathrm{KMS}_{\infty}$ states one finds only $\varphi(e)=0$, yet it is still possible to define an interesting action of endomorphisms by a procedure of "warming up and cooling down". For this to work one needs sufficiently favorable conditions, namely that the "warming up" map

$$
W_{\beta}(\varphi)(a)=\frac{\operatorname{Tr}\left(\pi_{\varphi}(a) e^{-\beta H}\right)}{\operatorname{Tr}\left(e^{-\beta H}\right)}
$$

gives a homeomorphism $W_{\beta}: \mathcal{E}_{\infty} \rightarrow \mathcal{E}_{\beta}$ for all $\beta$ sufficiently large. One can then define the action by

$$
\left(\rho^{*} \varphi\right)(a)=\lim _{\beta \rightarrow \infty}\left(\rho^{*} W_{\beta}(\varphi)\right)(a)
$$

for all $\varphi \in \mathcal{E}_{\infty}$ and all $a \in \mathcal{A}$.

\section{The Bost-Connes system}

We give the following geometric point of view on the $\mathrm{BC}$ system, following [38].

We use the notation $S h(G, X):=G(\mathbb{Q}) \backslash\left(G\left(\mathbb{A}_{f}\right) \times X\right)$ for Shimura varieties $(c f$. $[\mathbf{9 1}])$. Here $\mathbb{A}_{f}=\hat{\mathbb{Z}} \otimes \mathbb{Q}$ denotes the finite adèles of $\mathbb{Q}$, 
with $\hat{\mathbb{Z}}=\lim _{n} \mathbb{Z} / n \mathbb{Z}$. The simplest case is for $G=\mathrm{GL}_{1}$, where we consider

$$
\operatorname{Sh}\left(\mathrm{GL}_{1},\{ \pm 1\}\right)=\mathrm{GL}_{1}(\mathbb{Q}) \backslash\left(\mathrm{GL}_{1}\left(\mathbb{A}_{f}\right) \times\{ \pm 1\}\right)=\mathbb{Q}_{+}^{*} \backslash \mathbb{A}_{f}^{*}
$$

LEMMA 2.1. The quotient (3.12) parameterizes the invertible 1dimensional $\mathbb{Q}$-lattices up to scaling.

In fact, first notice that a 1-dimensional $\mathbb{Q}$-lattice can always be written in the form

$$
(\Lambda, \phi)=(\lambda \mathbb{Z}, \lambda \rho)
$$

for some $\lambda>0$ and some

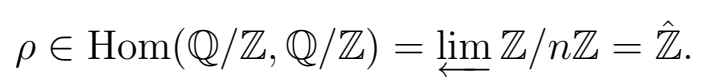

Thus, the set of 1-dimensional $\mathbb{Q}$-lattices up to scaling can be identified with $\hat{\mathbb{Z}}$. The invertible lattices correspond to the elements of $\hat{\mathbb{Z}}^{*}$, which in turn is identified with $\mathrm{GL}_{1}^{+}(\mathbb{Q}) \backslash \mathrm{GL}_{1}\left(\mathbb{A}_{f}\right)$.

The quotient (3.12) can be thought of geometrically as the Shimura variety associated to the cyclotomic tower $(c f .[38])$. This is the tower $\mathcal{V}$ of arithmetic varieties over $V=\operatorname{Spec}(\mathbb{Z})$, with $V_{n}=\operatorname{Spec}\left(\mathbb{Z}\left[\zeta_{n}\right]\right)$, where $\zeta_{n}$ is a primitive n-th root of unity. The group of deck transformations is $\operatorname{Aut}_{V}\left(V_{n}\right)=\mathrm{GL}_{1}(\mathbb{Z} / n \mathbb{Z})$, so that the group of deck transformations of the tower is the projective limit

$$
\operatorname{Aut}_{V}(\mathcal{V})=\overleftarrow{n}_{n}^{\lim } \operatorname{Aut}_{V}\left(V_{n}\right)=\mathrm{GL}_{1}(\hat{\mathbb{Z}}) \text {. }
$$

If, instead of invertible $\mathbb{Q}$-lattices up to scale, we consider the set of commensurability classes of all 1-dimensional $\mathbb{Q}$-lattices up to scale, we find a noncommutative version of the Shimura variety (3.12) and of the cyclotomic tower. This is described as the quotient

$$
S h^{(n c)}\left(\mathrm{GL}_{1},\{ \pm 1\}\right):=\mathrm{GL}_{1}(\mathbb{Q}) \backslash\left(\mathbb{A}_{f} \times\{ \pm 1\}\right)=\mathrm{GL}_{1}(\mathbb{Q}) \backslash \mathbb{A}^{*} / \mathbb{R}_{+}^{*}
$$

where $\mathbb{A}^{\cdot}:=\mathbb{A}_{f} \times \mathbb{R}^{*}$ are the adèles of $\mathbb{Q}$ with invertible archimedean component.

The corresponding noncommutative algebra of coordinates is given by the crossed product ( $c f .[6 \mathbf{6 8}])$

$$
C_{0}\left(\mathbb{A}_{f}\right) \rtimes \mathbb{Q}_{+}^{*} .
$$

One obtains an equivalent description of the noncommutative space (3.16), starting from the description (3.13) of 1-dimensional $\mathbb{Q}$-lattices. The commensurability relation is implemented by the action of $\mathbb{N}^{\times}=$ $\mathbb{Z}_{>0}$ by

$$
\alpha_{n}(f)(\rho)=\left\{\begin{array}{lr}
f\left(n^{-1} \rho\right) & \rho \in n \hat{\mathbb{Z}} \\
0 & \text { otherwise }
\end{array}\right.
$$




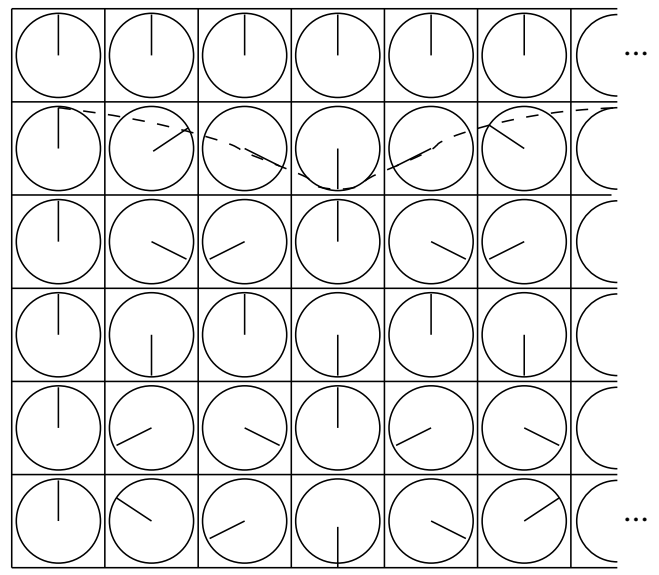

FiguRE 3. Phasors with $\mathbb{Z} / 6 \mathbb{Z}$ discretization

Thus, the noncommutative algebra of coordinates of the space of commensurability classes of 1-dimensional $\mathbb{Q}$-lattices up to scaling can be identified with the semigroup crossed product

$$
\mathcal{A}_{1}:=C^{*}(\mathbb{Q} / \mathbb{Z}) \rtimes \mathbb{N}^{\times},
$$

where we have identified

$$
C(\hat{\mathbb{Z}}) \simeq C^{*}(\mathbb{Q} / \mathbb{Z})
$$

since $\hat{\mathbb{Z}}$ is the Pontrjagin dual of $\mathbb{Q} / \mathbb{Z}$. The algebra (3.19) is Morita equivalent to the algebra (3.17) (cf. [68]).

The algebra (3.19) is isomorphic to the algebra considered in the work of Bost-Connes [9], which was obtained there as a Hecke algebra for the inclusion $\Gamma_{0} \subset \Gamma$ of the pair of groups $\left(\Gamma_{0}, \Gamma\right)=\left(P_{\mathbb{Z}}, P_{\mathbb{Q}}\right)$, where $P$ is the $a x+b$ group. These have the property that the left $\Gamma_{0}$ orbits of any $\gamma \in \Gamma / \Gamma_{0}$ are finite (same for right orbits on the left coset). The ratio of the lengths of left and right $\Gamma_{0}$ orbits determines a canonical time evolution $\sigma_{t}$ on the algebra $(c f .[\mathbf{9}])$.

The algebra of the Bost-Connes system has an explicit presentation in terms of two sets of operators. The first type consists of phase operators $e(r)$, parameterized by elements $r \in \mathbb{Q} / \mathbb{Z}$. These phase operators can be represented on the Fock space generated by occupation numbers $|n\rangle$ as the operators

$$
e(r)|n\rangle=\alpha\left(\zeta_{r}^{n}\right)|n\rangle .
$$

Here we denote by $\zeta_{a / b}=\zeta_{b}^{a}$ the abstract roots of unity generating $\mathbb{Q}^{c y c l}$ and by $\alpha: \mathbb{Q}^{c y c l} \hookrightarrow \mathbb{C}$ an embedding that identifies $\mathbb{Q}^{\text {cycl }}$ with the subfield of $\mathbb{C}$ generated by the concrete roots of unity. 
These are the phase operators used in the theory of quantum optics and optical coherence to model the phase quantum-mechanically ( $c f$. [72] [74]), as well as to model the phasors in quantum computing. They are based on the choice of a certain scale $N$ at which the phase is discretized (Figure 3). Namely, the quantized optical phase is defined as a state

$$
\left|\theta_{m, N}\right\rangle=e\left(\frac{m}{N+1}\right) \cdot v_{N},
$$

where $v_{N}$ is a superposition of occupation states

$$
v_{N}=\frac{1}{(N+1)^{1 / 2}} \sum_{n=0}^{N}|n\rangle .
$$

One needs then to ensure that the results are consistent over changes of scale.

The other operators that generate the Bost-Connes algebra can be thought of as implementing the changes of scales in the optical phases in a consistent way. These operators are isometries $\mu_{n}$ parameterized by positive integers $n \in \mathbb{N}^{\times}=\mathbb{Z}_{>0}$. The changes of scale are described by the action of the $\mu_{n}$ on the $e(r)$ by

$$
\mu_{n} e(r) \mu_{n}^{*}=\frac{1}{n} \sum_{n s=r} e(s) .
$$

In addition to this compatibility condition, the operators $e(r)$ and $\mu_{n}$ satisfy other simple relations.

These give a presentation of the algebra of the BC system of the form $([\mathbf{9}],[\mathbf{6 7}])$ :

- $\mu_{n}^{*} \mu_{n}=1$, for all $n \in \mathbb{N}^{\times}$,

- $\mu_{k} \mu_{n}=\mu_{k n}$, for all $k, n \in \mathbb{N}^{\times}$,

- $e(0)=1, e(r)^{*}=e(-r)$, and $e(r) e(s)=e(r+s)$, for all $r, s \in \mathbb{Q} / \mathbb{Z}$

- For all $n \in \mathbb{N}^{\times}$and all $r \in \mathbb{Q} / \mathbb{Z}$, the relation (3.22) holds.

This shows that the Bost-Connes algebra is isomorphic to the algebra $\mathcal{A}_{1}$ of (3.19).

In terms of this explicit presentation, the time evolution is of the form

$$
\sigma_{t}\left(\mu_{n}\right)=n^{i t} \mu_{n}, \quad \sigma_{t}(e(r))=e(r) .
$$

The space (3.16) can be compactified by replacing $\mathbb{A}^{\cdot}$ by $\mathbb{A}$, as in [23]. This gives the quotient

$$
\overline{S h^{(n c)}}\left(\mathrm{GL}_{1},\{ \pm 1\}\right)=\mathrm{GL}_{1}(\mathbb{Q}) \backslash \mathbb{A} / \mathbb{R}_{+}^{*} .
$$


This compactification consists of adding the trivial lattice (with a possibly nontrivial $\mathbb{Q}$-structure).

The dual space to (3.24), under the duality of type II and type III factors introduced in Connes' thesis, is a principal $\mathbb{R}_{+}^{*}$-bundle over (3.24), whose noncommutative algebra of coordinates is obtained from the algebra of (3.24) by taking the crossed product by the time evolution $\sigma_{t}$. The space obtained this way is the space of adèle classes

$$
\mathcal{L}_{1}=\mathrm{GL}_{1}(\mathbb{Q}) \backslash \mathbb{A} \rightarrow \mathrm{GL}_{1}(\mathbb{Q}) \backslash \mathbb{A} / \mathbb{R}_{+}^{*}
$$

that gives the spectral realization of zeros of the Riemann $\zeta$ function in [23]. Passing to this dual space corresponds to considering commensurability classes of 1-dimensional $\mathbb{Q}$-lattices (no longer up to scaling).

2.1. Structure of KMS states. The Bost-Connes algebra $\mathcal{A}_{1}$ has irreducible representations on the Hilbert space $\mathcal{H}=\ell^{2}\left(\mathbb{N}^{\times}\right)$. These are parameterized by elements $\alpha \in \hat{\mathbb{Z}}^{*}=\mathrm{GL}_{1}(\hat{\mathbb{Z}})$. Any such element defines an embedding $\alpha: \mathbb{Q}^{c y c l} \hookrightarrow \mathbb{C}$ and the corresponding representation is of the form

$$
\begin{aligned}
\pi_{\alpha}(e(r)) \epsilon_{k} & =\alpha\left(\zeta_{r}^{k}\right) \epsilon_{k} \\
\pi_{\alpha}\left(\mu_{n}\right) \epsilon_{k} & =\epsilon_{n k}
\end{aligned}
$$

The Hamiltonian implementing the time evolution (3.18) on $\mathcal{H}$ is

$$
H \epsilon_{k}=(\log k) \epsilon_{k}
$$

Thus, the partition function of the Bost-Connes system is the Riemann zeta function

$$
Z(\beta)=\operatorname{Tr}\left(e^{-\beta H}\right)=\sum_{k=1}^{\infty} k^{-\beta}=\zeta(\beta) .
$$

Bost and Connes showed in [9] that KMS states have the following structure, with a phase transition at $\beta=1$.

- In the range $\beta \leq 1$ there is a unique $\mathrm{KMS}_{\beta}$ state. Its restriction to $\mathbb{Q}[\mathbb{Q} / \mathbb{Z}]$ is of the form

$$
\varphi_{\beta}(e(a / b))=b^{-\beta} \prod_{p \text { prime }, p \mid b} \frac{1-p^{\beta-1}}{1-p^{-1}} .
$$

- For $1<\beta \leq \infty$ the set of extremal KMS states $\mathcal{E}_{\beta}$ can be identified with $\hat{\mathbb{Z}}^{*}$. It has a free and transitive action of this group induced by an action on $\mathcal{A}$ by automorphisms. The extremal $\mathrm{KMS}_{\beta}$ state corresponding to $\alpha \in \hat{\mathbb{Z}}^{*}$ is of the form

$$
\varphi_{\beta, \alpha}(x)=\frac{1}{\zeta(\beta)} \operatorname{Tr}\left(\pi_{\alpha}(x) e^{-\beta H}\right) .
$$


- At $\beta=\infty$ the Galois group $\operatorname{Gal}\left(\mathbb{Q}^{c y c l} / \mathbb{Q}\right)$ acts on the values of states $\varphi \in \mathcal{E}_{\infty}$ on an arithmetic subalgebra $\mathcal{A}_{1, \mathbb{Q}} \subset \mathcal{A}_{1}$. These have the property that $\varphi\left(\mathcal{A}_{\mathbb{Q}}\right) \subset \mathbb{Q}^{\text {cycl }}$ and that the isomorphism (class field theory isomorphism) $\theta: \operatorname{Gal}\left(\mathbb{Q}^{\text {cycl }} / \mathbb{Q}\right) \cong \hat{\mathbb{Z}}^{*}$ intertwines the Galois action on values with the action of $\hat{\mathbb{Z}}^{*}$ by symmetries, namely,

$$
\gamma \varphi(x)=\varphi(\theta(\gamma) x)
$$

for all $\varphi \in \mathcal{E}_{\infty}$, for all $\gamma \in \operatorname{Gal}\left(\mathbb{Q}^{c y c l} / \mathbb{Q}\right)$ and for all $x \in \mathcal{A}_{\mathbb{Q}}$.

Here the arithmetic subalgebra $\mathcal{A}_{1, \mathbb{Q}}$ can be taken to be the algebra over $\mathbb{Q}$ generated by the $e(r)$ and the $\mu_{n}, \mu_{n}^{*}$. This $\mathbb{Q}$-algebra can also be obtained (as shown in [32]) as the algebra generated by the $\mu_{n}, \mu_{n}^{*}$ and by homogeneous functions of weight zero on 1-dim $\mathbb{Q}$-lattices obtained as a normalization of the functions

$$
\epsilon_{k, a}(\Lambda, \phi)=\sum_{y \in \Lambda+\phi(a)} y^{-k}
$$

by covolume. Namely, one considers the functions $e_{k, a}:=c^{k} \epsilon_{k, a}$, where $c(\Lambda)$ is proportional to the covolume $|\Lambda|$ and satisfies

$$
(2 \pi \sqrt{-1}) c(\mathbb{Z})=1 .
$$

The choice of an "arithmetic subalgebra" corresponds to endowing the noncommutative space $\mathcal{A}$ with an arithmetic structure. The subalgebra corresponds to the rational functions and the values of $\mathrm{KMS}_{\infty}$ states at elements of this subalgebra should be thought of as values of "rational functions" at the classical points of the noncommutative space $(c f .[38])$.

\section{Noncommutative Geometry and Hilbert's 12th problem}

The most remarkable arithmetic feature of the result of Bost-Connes recalled above is the Galois action on the ground states of the system. First of all, the fact that the Galois action on the values of states would preserve positivity (i.e. would give values of other states) is a very unusual property. Moreover, the values of extremal ground states on elements of the rational subalgebra generate the maximal abelian extension $\mathbb{Q}^{a b}=\mathbb{Q}^{c y c l}$ of $\mathbb{Q}$. The explicit action of the Galois $\operatorname{group} \operatorname{Gal}\left(\mathbb{Q}^{a b} / \mathbb{Q}\right) \simeq \mathrm{GL}_{1}(\hat{\mathbb{Z}})$ is given by automorphisms of the system $\left(\mathcal{A}_{1}, \sigma_{t}\right)$. Namely, the class field theory isomorphism intertwines the action of the idèle class group, as symmetry group of the system, and that of the Galois group, as permutations of the expectation values of the rational observables. 
In general, the main theorem of class field theory provides a classification of finite abelian extensions of a local or global field $\mathbb{K}$ in terms of subgroups of a locally compact abelian group canonically associated to the field. This is the multiplicative group $\mathbb{K}^{*}=\mathrm{GL}_{1}(\mathbb{K})$ in the local nonarchimedean case, while in the global case it is the quotient $C_{\mathbb{K}} / D_{\mathbb{K}}$ of the idèle class group $C_{\mathbb{K}}$ by the connected component of the identity.

Hilbert's 12th problem can be formulated as the question of providing an explicit set of generators of the maximal abelian extension $\mathbb{K}^{a b}$ of a number field $\mathbb{K}$, inside an algebraic closure $\overline{\mathbb{K}}$, and of the action of the Galois group $\operatorname{Gal}\left(\mathbb{K}^{a b} / \mathbb{K}\right)$. The maximal abelian extension $\mathbb{K}^{a b}$ of $\mathbb{K}$ has the property that

$$
\operatorname{Gal}\left(\mathbb{K}^{a b} / \mathbb{K}\right)=\operatorname{Gal}(\overline{\mathbb{K}} / \mathbb{K})^{a b}
$$

The result that motivated Hilbert's formulation of the explicit class field theory problem is the Kronecker-Weber theorem, namely the already mentioned case of the explicit class field theory of $\mathbb{K}=\mathbb{Q}$. In this case, the maximal abelian extension of $\mathbb{Q}$ can be identified with the cyclotomic field $\mathbb{Q}^{c y c l}$. Equivalently, one can say that the torsion points of the multiplicative group $\mathbb{C}^{*}$ (i.e. the roots of unity) generate $\mathbb{Q}^{a b} \subset \mathbb{C}$.

Remarkably, the only other case for number fields where this program has been carried out completely is that of imaginary quadratic fields, where the construction relies on the theory of elliptic curves with complex multiplication, and on the Galois theory of the field of modular functions ( $c f$. e.g. the survey [106]).

Some generalizations of the original result of Bost-Connes to other global fields (number fields and function fields) were obtained by Harari and Leichtnam [60], P. Cohen [15], Arledge, Laca and Raeburn [4]. A more detailed account of various results related to the BC system and generalizations is given in the "further developments" section of [32]. Because of this close relation to Hilbert's 12th problem, it is clear that finding generalizations of the Bost-Connes system to other number fields is a very difficult problem, hence it is not surprising that, so far, these constructions have not fully recovered the Galois properties of the ground states of the BC system in the generalized setting.

The strongest form of the result one may wish to obtain in this direction can be formulated as follows.

Given a number field $\mathbb{K}$, we let $\mathbb{A}_{\mathbb{K}}$ denote the adèles of $\mathbb{K}$ and we let $\mathbb{A}_{\mathbb{K}}^{*}=\mathrm{GL}_{1}\left(\mathbb{A}_{\mathbb{K}}\right)$ be the group of idèles of $\mathbb{K}$. As above, we write $C_{\mathbb{K}}$ for the group of idèle classes $C_{\mathbb{K}}=\mathbb{A}_{\mathbb{K}}^{*} / \mathbb{K}^{*}$ and $D_{\mathbb{K}}$ for the connected component of the identity in $C_{\mathbb{K}}$. 
One wishes to construct a $C^{*}$-dynamical system $\left(\mathcal{A}_{\mathbb{K}}, \sigma_{t}\right)$ and an "arithmetic" subalgebra $\mathcal{A}_{\mathbb{K}, \mathbb{Q}}$, which satisfy the following properties:

(1) The idèle class group $G=C_{\mathbb{K}} / D_{\mathbb{K}}$ acts by symmetries on $\left(\mathcal{A}_{\mathbb{K}}, \sigma_{t}\right)$ preserving the subalgebra $\mathcal{A}_{\mathbb{K}, \mathbb{Q}}$.

(2) The states $\varphi \in \mathcal{E}_{\infty}$, evaluated on elements of $\mathcal{A}_{\mathbb{K}, \mathbb{Q}}$, satisfy:

- $\varphi(a) \in \mathbb{K}$, the algebraic closure of $\mathbb{K}$ in $\mathbb{C}$;

- the elements of $\left\{\varphi(a): a \in \mathcal{A}_{\mathbb{K}, \mathbb{Q}}\right\}$, for $\varphi \in \mathcal{E}_{\infty}$ generate $\mathbb{K}^{a b}$.

(3) The class field theory isomorphism

$$
\theta: \operatorname{Gal}\left(\mathbb{K}^{a b} / \mathbb{K}\right) \stackrel{\simeq}{\longrightarrow} C_{\mathbb{K}} / D_{\mathbb{K}}
$$

intertwines the actions,

$$
\alpha \circ \varphi=\varphi \circ \theta(\alpha),
$$

for all $\alpha \in \operatorname{Gal}\left(\mathbb{K}^{a b} / \mathbb{K}\right)$ and for all $\varphi \in \mathcal{E}_{\infty}$.

The arithmetic subalgebra $\mathcal{A}_{\mathbb{K}, \mathbb{Q}}$, in general, need not be an involutive subalgebra.

The setup described above may provide a possible new approach, via noncommutative geometry, to the explicit class field theory problem.

Given a number field $\mathbb{K}$ with $[\mathbb{K}: \mathbb{Q}]=n$, there is an embedding $\mathbb{K}^{*} \hookrightarrow \mathrm{GL}_{n}(\mathbb{Q})$ of its multiplicative group in $\mathrm{GL}_{n}(\mathbb{Q})$. Such embedding induces an embedding of $\mathrm{GL}_{1}\left(\mathbb{A}_{\mathbb{K}, f}\right)$ into $\mathrm{GL}_{n}\left(\mathbb{A}_{f}\right)$, where $\mathbb{A}_{\mathbb{K}, f}=\mathbb{A}_{f} \otimes \mathbb{K}$ are the finite adèles of $\mathbb{K}$.

This suggests that a possible strategy to approach the problem stated above may be to first study quantum statistical mechanical systems corresponding to $\mathrm{GL}_{n}$ analogs of the Bost-Connes system.

The main result of the joint work of Alain Connes and the author in [32] is the construction of such a system in the $\mathrm{GL}_{2}$ case and the analysis of the arithmetic properties of its KMS states. In the case of $\mathrm{GL}_{2}$, one sees that the geometry of modular curves and the algebra of modular forms appear naturally.

In joint work of Alain Connes, Niranjan Ramachandran, and the author [38], a quantum statistical mechanical system is constructed, which satisfies all the properties listed above for the case where $\mathbb{K}$ is an imaginary quadratic field. The properties of this system are intermediate between those of the original Bost-Connes system and those of the $\mathrm{GL}_{2}$ system of $[32]$. In fact, geometrically the construction is based on replacing the 1-dimensional $\mathbb{Q}$-lattices of the Bost-Connes system with 1-dimensional $\mathbb{K}$-lattices. The groupoid of the commensurability relation is then a subgroupoid of that of the $\mathrm{GL}_{2}$-system (1-dimensional 
$\mathbb{K}$-lattices are a special class of 2-dimensional $\mathbb{Q}$-lattices with compatible notions of commensurability). Thus, by combining the techniques of the Bost-Connes and of the $\mathrm{GL}_{2}$ system, it is possible to show that the complex multiplication (CM) system defined this way recovers the full picture of the explicit class field theory of imaginary quadratic fields [38], as we will recall in this chapter.

The first case for which there is not yet a complete solution to the explicit class field theory problem is the case of real quadratic fields, $\mathbb{K}=\mathbb{Q}(\sqrt{d})$, for some positive integer $d$. It is natural to ask whether the approach outlined above, based on noncommutative geometry, may provide any new information on this case. Recent work of Manin [75] [76] suggests a close relation between the real quadratic case and noncommutative geometry. We will discuss the possible relation between his approach and the $\mathrm{GL}_{2}$-system.

\section{The $\mathrm{GL}_{2}$ system}

In this section we will describe the main features of the $\mathrm{GL}_{2}$ analog of the Bost-Connes system, according to the results of [32].

We can start with the same geometric approach in terms of Shimura varieties $(c f .[38],[33])$ that we used above to introduce the $\mathrm{BC}$ system.

The Shimura variety associated to the tower of modular curves is described by the adelic quotient

$$
\begin{aligned}
\operatorname{Sh}\left(\mathrm{GL}_{2}, \mathbb{H}^{ \pm}\right) & =\mathrm{GL}_{2}(\mathbb{Q}) \backslash\left(\mathrm{GL}_{2}\left(\mathbb{A}_{f}\right) \times \mathbb{H}^{ \pm}\right) \\
& =\mathrm{GL}_{2}^{+}(\mathbb{Q}) \backslash\left(\mathrm{GL}_{2}\left(\mathbb{A}_{f}\right) \times \mathbb{H}\right)=\mathrm{GL}_{2}^{+}(\mathbb{Q}) \backslash \mathrm{GL}_{2}(\mathbb{A}) / \mathbb{C}^{*}
\end{aligned}
$$

The tower of modular curves $(c f$. $[38])$ has base $V=\mathbb{P}^{1}$ over $\mathbb{Q}$ and $V_{n}=X(n)$ the modular curve corresponding to the principal congruence subgroup $\Gamma(n)$. The automorphisms of the projection $V_{n} \rightarrow V$ are given by $\mathrm{GL}_{2}(\mathbb{Z} / n \mathbb{Z}) /\{ \pm 1\}$, so that the group of deck transformations of the tower $\mathcal{V}$ is in this case of the form

$$
\operatorname{Aut}_{V}(\mathcal{V})={\underset{n}{\lim }}_{\mathrm{GL}_{2}}(\mathbb{Z} / n \mathbb{Z}) /\{ \pm 1\}=\mathrm{GL}_{2}(\hat{\mathbb{Z}}) /\{ \pm 1\} .
$$

The inverse limit $\lim _{\longleftarrow} \Gamma \mathbb{H}$ over congruence subgroups $\Gamma \subset \operatorname{SL}(2, \mathbb{Z})$ gives a connected component of (3.33), while by taking congruence subgroups in $\mathrm{SL}(2, \mathbb{Q})$ one obtains the adelic version $S h\left(\mathrm{GL}_{2}, \mathbb{H}^{ \pm}\right)$. The simple reason why it is necessary to pass to the nonconnected case is the following. The varieties in the tower are arithmetic varieties defined over number fields. However, the number field typically changes along the levels of the tower $\left(V_{n}\right.$ is defined over the cyclotomic field $\mathbb{Q}\left(\zeta_{n}\right)$ ). Passing to nonconnected Shimura varieties allows for the definition of a 
canonical model where the whole tower is defined over the same number field.

The quotient (3.33) parameterizes invertible 2-dimensional $\mathbb{Q}$-lattices up to scaling $(c f .[\mathbf{9 1}])$. When, instead of restricting to the invertible case, we consider commensurability classes of 2-dimensional $\mathbb{Q}$-lattices up to scaling, we obtain a noncommutative space whose classical points are the Shimura variety (3.33). More precisely, we have the following $(c f .[38])$ :

LEMMA 4.1. The space of commensurability classes of 2-dimensional $\mathbb{Q}$-lattices up to scaling is described by the quotient

$$
S h^{(n c)}\left(\mathrm{GL}_{2}, \mathbb{H}^{ \pm}\right):=\mathrm{GL}_{2}(\mathbb{Q}) \backslash\left(M_{2}\left(\mathbb{A}_{f}\right) \times \mathbb{H}^{ \pm}\right) .
$$

Any 2-dimensional $\mathbb{Q}$-lattice can be written in the form

$$
(\Lambda, \phi)=(\lambda(\mathbb{Z}+\mathbb{Z} \tau), \lambda \rho),
$$

for some $\lambda \in \mathbb{C}^{*}$, some $\tau \in \mathbb{H}$, and some

$$
\rho \in M_{2}(\hat{\mathbb{Z}})=\operatorname{Hom}\left(\mathbb{Q}^{2} / \mathbb{Z}^{2}, \mathbb{Q}^{2} / \mathbb{Z}^{2}\right) .
$$

Thus, the space of 2-dimensional $\mathbb{Q}$-lattices up to the scale factor $\lambda \in$ $\mathbb{C}^{*}$ and up to isomorphisms, is given by

$$
M_{2}(\hat{\mathbb{Z}}) \times \mathbb{H} \quad \bmod \Gamma=\mathrm{SL}(2, \mathbb{Z}) .
$$

The commensurability relation is implemented by the partially defined action of $\mathrm{GL}_{2}^{+}(\mathbb{Q})$ on this space, given by

$$
g(\rho, z)=(g \rho, g(z)),
$$

where $g(z)$ denotes action as fractional linear transformation.

Commensurability classes of $\mathbb{Q}$-lattices $(\Lambda, \phi)$ in $\mathbb{C}$ are equivalent to isogeny classes of pairs $(E, \eta)$ of an elliptic curve $E=\mathbb{C} / \Lambda$ and an $\mathbb{A}_{f}$-homomorphism

$$
\eta: \mathbb{Q}^{2} \otimes \mathbb{A}_{f} \rightarrow \Lambda \otimes \mathbb{A}_{f}
$$

where $\Lambda \otimes \mathbb{A}_{f}=(\Lambda \otimes \hat{\mathbb{Z}}) \otimes \mathbb{Q}$, with

$$
\Lambda \otimes \hat{\mathbb{Z}}={\underset{n}{\lim }}_{\lim } \Lambda / n \Lambda,
$$

and $\Lambda / n \Lambda=E[n]$ the $n$-torsion of $E$. Since the $\mathbb{Q}$-lattices need not be invertible, we do not require that $\eta$ be an $\mathbb{A}_{f}$-isomorphism $(c f .[\mathbf{9 1}])$. The commensurability relation between $\mathbb{Q}$-lattices corresponds to the equivalence $(E, \eta) \sim\left(E^{\prime}, \eta^{\prime}\right)$ given by an isogeny $g: E \rightarrow E^{\prime}$ and $\eta^{\prime}=$ $(g \otimes 1) \circ \eta$. Namely, the equivalence classes can be identified with the quotient of $M_{2}\left(\mathbb{A}_{f}\right) \times \mathbb{H}^{ \pm}$by the action of $\mathrm{GL}_{2}(\mathbb{Q}),(\rho, z) \mapsto(g \rho, g(z))$. 
To associate a quantum statistical mechanical system to the space of commensurability classes of 2 -dimensional $\mathbb{Q}$-lattices up to scaling, it is convenient to use the description (3.36). Then one can consider as noncommutative algebra of coordinates the convolution algebra of continuous compactly supported functions on the quotient of the space

$$
\mathcal{U}:=\left\{(g, \rho, z) \in \mathrm{GL}_{2}^{+}(\mathbb{Q}) \times M_{2}(\hat{\mathbb{Z}}) \times \mathbb{H} \mid g \rho \in M_{2}(\hat{\mathbb{Z}})\right\}
$$

by the action of $\Gamma \times \Gamma$,

$$
(g, \rho, z) \mapsto\left(\gamma_{1} g \gamma_{2}^{-1}, \gamma_{2} \rho, \gamma_{2}(z)\right)
$$

One endows this algebra with the convolution product

$$
\left(f_{1} * f_{2}\right)(g, \rho, z)=\sum_{s \in \Gamma \backslash \mathrm{GL}_{2}^{+}(\mathbb{Q}): s \rho \in M_{2}(\hat{\mathbb{Z}})} f_{1}\left(g s^{-1}, s \rho, s(z)\right) f_{2}(s, \rho, z)
$$

and the involution $f^{*}(g, \rho, z)=\overline{f\left(g^{-1}, g \rho, g(z)\right)}$.

The time evolution is given by

$$
\sigma_{t}(f)(g, \rho, z)=\operatorname{det}(g)^{i t} f(g, \rho, z) .
$$

For $\rho \in M_{2}(\hat{\mathbb{Z}})$ let

$$
G_{\rho}:=\left\{g \in \mathrm{GL}_{2}^{+}(\mathbb{Q}): g \rho \in M_{2}(\hat{\mathbb{Z}})\right\}
$$

and consider the Hilbert space $\mathcal{H}_{\rho}=\ell^{2}\left(\Gamma \backslash G_{\rho}\right)$.

A 2-dimensional $\mathbb{Q}$-lattice $L=(\Lambda, \phi)=(\rho, z)$ determines a representation of the Hecke algebra by bounded operators on $\mathcal{H}_{\rho}$, setting

$$
\left(\pi_{L}(f) \xi\right)(g)=\sum_{s \in \Gamma \backslash G_{\rho}} f\left(g s^{-1}, s \rho, s(z)\right) \xi(s) .
$$

In particular, when the $\mathbb{Q}$-lattice $L=(\Lambda, \phi)$ is invertible one obtains

$$
\mathcal{H}_{\rho} \cong \ell^{2}\left(\Gamma \backslash M_{2}^{+}(\mathbb{Z})\right) .
$$

In this case, the Hamiltonian implementing the time evolution (3.42) is given by the operator

$$
H \epsilon_{m}=\log \operatorname{det}(m) \epsilon_{m} .
$$

Thus, in the special case of invertible $\mathbb{Q}$-lattices (3.44) yields a positive energy representation. In general for $\mathbb{Q}$-lattices which are not commensurable to an invertible one, the corresponding Hamiltonian $H$ is not bounded below.

The Hecke algebra (3.41) admits a $C^{*}$-algebra completion $\mathcal{A}_{2}$, where the norm is the sup over all representations $\pi_{L}$. 
The partition function for this $\mathrm{GL}_{2}$ system is given by

$$
Z(\beta)=\sum_{m \in \Gamma \backslash M_{2}^{+}(\mathbb{Z})} \operatorname{det}(m)^{-\beta}=\sum_{k=1}^{\infty} \sigma(k) k^{-\beta}=\zeta(\beta) \zeta(\beta-1),
$$

where $\sigma(k)=\sum_{d \mid k} d$. This suggests the fact that one expects two phase transitions to take place, at $\beta=1$ and $\beta=2$, respectively.

The set of components of $S h\left(\mathrm{GL}_{2}, \mathbb{H}^{ \pm}\right)$is given by

$$
\pi_{0}\left(S h\left(\mathrm{GL}_{2}, \mathbb{H}^{ \pm}\right)\right)=S h\left(\mathrm{GL}_{1},\{ \pm 1\}\right) .
$$

At the level of the classical commutative spaces, this is given by the map

$$
\operatorname{det} \times \operatorname{sign}: S h\left(\mathrm{GL}_{2}, \mathbb{H}^{ \pm}\right) \rightarrow S h\left(\mathrm{GL}_{1},\{ \pm 1\}\right),
$$

which corresponds in fact to passing to the set $\pi_{0}$ of connected components.

4.1. KMS states of the $\mathrm{GL}_{2}$-system. The main result of $[32]$ on the structure of KMS states is the following.

ThEOREM 4.2. The $K M S_{\beta}$ states of the $\mathrm{GL}_{2}$-system have the following properties:

(1) In the range $\beta \leq 1$ there are no KMS states.

(2) In the range $\beta>2$ the set of extremal KMS states is given by the classical Shimura variety

$$
\mathcal{E}_{\beta} \cong \mathrm{GL}_{2}(\mathbb{Q}) \backslash \mathrm{GL}_{2}(\mathbb{A}) / \mathbb{C}^{*} .
$$

This shows that the extremal KMS states at sufficiently low temperature are parameterized by the invertible $\mathbb{Q}$-lattices. The explicit expression for these extremal $\mathrm{KMS}_{\beta}$ states is obtained as

$$
\varphi_{\beta, L}(f)=\frac{1}{Z(\beta)} \sum_{m \in \Gamma \backslash M_{2}^{+}(\mathbb{Z})} f(1, m \rho, m(z)) \operatorname{det}(m)^{-\beta}
$$

where $L=(\rho, z)$ is an invertible $\mathbb{Q}$-lattice.

As the temperature rises, and we let $\beta \rightarrow 2$ from above, all the different phases of the system merge, which is strong evidence for the fact that in the intermediate range $1<\beta \leq 2$ the system should have only a single KMS state.

The symmetry group of $\mathcal{A}_{2}$ (including both automorphisms and endomorphisms) can be identified with the group

$$
\mathrm{GL}_{2}\left(\mathbb{A}_{f}\right)=\mathrm{GL}_{2}^{+}(\mathbb{Q}) \mathrm{GL}_{2}(\hat{\mathbb{Z}}) \text {. }
$$


Here the group $\mathrm{GL}_{2}(\hat{\mathbb{Z}})$ acts by automorphisms,

$$
\theta_{\gamma}(f)(g, \rho, z)=f(g, \rho \gamma, z) .
$$

These symmetries are related to deck transformations (3.34) of coverings of modular curves.

The novelty with respect to the $\mathrm{BC}$ case is that we also have an action of $\mathrm{GL}_{2}^{+}(\mathbb{Q})$ by endomorphisms

$$
\theta_{m}(f)(g, \rho, z)=\left\{\begin{array}{lr}
f\left(g, \rho \tilde{m}^{-1}, z\right) & \rho \in m M_{2}(\hat{\mathbb{Z}}) \\
0 & \text { otherwise }
\end{array}\right.
$$

where $\tilde{m}=\operatorname{det}(m) m^{-1}$.

The subgroup $\mathbb{Q}^{*} \hookrightarrow \mathrm{GL}_{2}\left(\mathbb{A}_{f}\right)$ acts by inner endomorphisms, hence the group of symmetries of the set of extremal states $\mathcal{E}_{\beta}$ is of the form

$$
S=\mathbb{Q}^{*} \backslash \mathrm{GL}_{2}\left(\mathbb{A}_{f}\right) \text {. }
$$

In the case of $\mathcal{E}_{\infty}$ states (defined as weak limits) the action of $\mathrm{GL}_{2}^{+}(\mathbb{Q})$ is more subtle to define. In fact, (3.53) does not directly induce a nontrivial action on $\mathcal{E}_{\infty}$. However, there is a nontrivial action induced by the action on $\mathcal{E}_{\beta}$ states for sufficiently large $\beta$. The action on the $\mathrm{KMS}_{\infty}$ states is obtained by a "warming up and cooling down procedure", as in (3.10) and (3.11).

Finally, the Galois action on the extremal $\mathrm{KMS}_{\infty}$ states is described by the following result [32].

THEOREM 4.3. There exists an arithmetic subalgebra $\mathcal{A}_{2, \mathbb{Q}}$ of unbounded multiplier of the $C^{*}$-algebra $\mathcal{A}_{2}$, such that the following holds. For $\varphi_{\infty, L} \in \mathcal{E}_{\infty}$ with $L=(\rho, \tau)$ generic, the values on arithmetic elements satisfy

$$
\varphi_{\infty, L}\left(\mathcal{A}_{2, \mathbb{Q}}\right) \subset F_{\tau},
$$

where $F_{\tau}$ is the embedding of the modular field in $\mathbb{C}$ given by evaluation at $\tau \in \mathbb{H}$. The values (3.55) generate $F_{\tau}$. There is an isomorphism

$$
\theta_{\varphi}: \operatorname{Gal}\left(F_{\tau} / \mathbb{Q}\right) \stackrel{\simeq}{\longrightarrow} \mathbb{Q}^{*} \backslash \mathrm{GL}_{2}\left(\mathbb{A}_{f}\right),
$$

that intertwines the Galois action on the values of the state with the action of symmetries,

$$
\gamma \varphi(f)=\varphi\left(\theta_{\varphi}(\gamma) f\right), \quad \forall f \in \mathcal{A}_{2, \mathbb{Q}}, \quad \forall \gamma \in \operatorname{Gal}\left(F_{\tau} / \mathbb{Q}\right) .
$$

Here recall that the modular field $F$ is the field of modular functions over $\mathbb{Q}^{a b}$, namely the union of the fields $F_{N}$ of modular functions of level $N$ rational over the cyclotomic field $\mathbb{Q}\left(\zeta_{n}\right)$, that is, such that the $q$-expansion in powers of $q^{1 / N}=\exp (2 \pi i \tau / N)$ has all coefficients in $\mathbb{Q}\left(e^{2 \pi i / N}\right)$. 
It has explicit generators given by the Fricke functions ([104], [69]). If $\wp$ is the Weierstrass $\wp$-function, which gives the parameterization

$$
z \mapsto\left(1, \wp(z ; \tau, 1), \wp^{\prime}(z ; \tau, 1)\right)
$$

of the elliptic curve

$$
y^{2}=4 x^{3}-g_{2}(\tau) x-g_{3}(\tau)
$$

by the quotient $\mathbb{C} /(\mathbb{Z}+\mathbb{Z} \tau)$, then the Fricke functions are homogeneous functions of 1-dimensional lattices of weight zero, parameterized by $v \in \mathbb{Q}^{2} / \mathbb{Z}^{2}$, of the form

$$
f_{v}(\tau)=-2^{7} 3^{5} \frac{g_{2}(\tau) g_{3}(\tau)}{\Delta(\tau)} \wp\left(\lambda_{\tau}(v) ; \tau, 1\right),
$$

where $\Delta=g_{2}^{3}-27 g_{3}^{2}$ is the discriminant and $\lambda_{\tau}(v):=v_{1} \tau+v_{2}$.

For generic $\tau$ (such that $j(\tau)$ is transcendental), evaluation of the modular functions at the point $\tau \in \mathbb{H}$ gives an embedding $F_{\tau} \hookrightarrow \mathbb{C}$. There is a corresponding isomorphism

$$
\theta_{\tau}: \operatorname{Gal}\left(F_{\tau} / \mathbb{Q}\right) \stackrel{\simeq}{\rightarrow} \operatorname{Aut}(F) .
$$

The isomorphism (3.56) is given by

$$
\theta_{\varphi}(\gamma)=\rho^{-1} \theta_{\tau}(\gamma) \rho
$$

for $\theta_{\tau}$ as in (3.59). In fact, the group of automorphisms $\operatorname{Aut}(F)$ has a completely explicit description, due to a result of Shimura [104], which identifies it with the quotient

$$
\operatorname{Aut}(F) \cong \mathbb{Q}^{*} \backslash \mathrm{GL}_{2}\left(\mathbb{A}_{f}\right) .
$$

We still need to explain what is the arithmetic subalgebra that appears in Theorem 4.3.

We consider continuous functions on the quotient of (3.39) by the action (3.40), which have finite support in the variable $g \in \Gamma \backslash \mathrm{GL}_{2}^{+}(\mathbb{Q})$. For convenience we adopt the notation

$$
f_{(g, \rho)}(z)=f(g, \rho, z)
$$

so that $f_{(g, \rho)} \in C(\mathbb{H})$. Let $p_{N}: M_{2}(\hat{\mathbb{Z}}) \rightarrow M_{2}(\mathbb{Z} / N \mathbb{Z})$ be the canonical projection. We say that $f$ is of level $N$ if

$$
f_{(g, \rho)}=f_{\left(g, p_{N}(\rho)\right)} \quad \forall(g, \rho) .
$$

Then $f$ is completely determined by the functions

$$
f_{(g, m)} \in C(\mathbb{H}), \quad \text { for } m \in M_{2}(\mathbb{Z} / N \mathbb{Z}) .
$$

Notice that the invariance

$$
f(g \gamma, \rho, z)=f(g, \gamma \rho, \gamma(z)),
$$


for all $\gamma \in \Gamma$ and for all $(g, \rho, z) \in \mathcal{U}$, implies that we have

$$
f_{(g, m) \mid \gamma}=f_{(g, m)}, \quad \forall \gamma \in \Gamma(N) \cap g^{-1} \Gamma g .
$$

so that $f$ is invariant under a congruence subgroup.

Elements $f$ of $\mathcal{A}_{2, \mathbb{Q}}$ are characterized by the following properties.

- The support of $f$ in $\Gamma \backslash \mathrm{GL}_{2}^{+}(\mathbb{Q})$ is finite.

- The function $f$ is of finite level with

$$
f_{(g, m)} \in F \quad \forall(g, m) .
$$

- The function $f$ satisfies the cyclotomic condition:

$$
f_{(g, \alpha(u) m)}=\operatorname{cycl}(u) f_{(g, m)},
$$

for all $g \in \mathrm{GL}_{2}^{+}(\mathbb{Q})$ diagonal and all $u \in \hat{\mathbb{Z}}^{*}$, with

$$
\alpha(u)=\left(\begin{array}{ll}
u & 0 \\
0 & 1
\end{array}\right) .
$$

Here cycl $: \hat{\mathbb{Z}}^{*} \rightarrow \operatorname{Aut}(F)$ denotes the action of the Galois group $\hat{\mathbb{Z}}^{*} \simeq$ $\operatorname{Gal}\left(\mathbb{Q}^{a b} / \mathbb{Q}\right)$ on the coefficients of the $q$-expansion in powers of $q^{1 / N}=$ $\exp (2 \pi i \tau / N)$.

If we took only the first two conditions, this would allow the algebra $\mathcal{A}_{2, \mathbb{Q}}$ to contain the cyclotomic field $\mathbb{Q}^{a b} \subset \mathbb{C}$, but this would prevent the existence of states satisfying the desired Galois property. In fact, if the subalgebra contains scalar elements in $\mathbb{Q}^{\text {cycl }}$, the sought for Galois property would not be compatible with the $\mathbb{C}$-linearity of states. The cyclotomic condition then forces the spectrum of the elements of $\mathcal{A}_{2, \mathbb{Q}}$ to contain all Galois conjugates of any root of unity that appears in the coefficients of the $q$-series, so that elements of $\mathcal{A}_{2, \mathbb{Q}}$ cannot be scalars.

The algebra $\mathcal{A}_{2, \mathbb{Q}}$ defined by the properties above is a subalgebra of unbounded multipliers of $\mathcal{A}_{2}$, which is globally invariant under the group of symmetries $\mathbb{Q}^{*} \backslash \mathrm{GL}_{2}\left(\mathbb{A}_{f}\right)$.

\section{Quadratic fields}

The first essential step in the direction of generalizing the BC system to other number fields and exploring a possible approach to the explicit class field theory problem via noncommutative geometry is the construction of a system that recovers the explicit class field theory for imaginary quadratic fields $\mathbb{K}=\mathbb{Q}(\sqrt{-d})$, for $d$ a positive integer.

We will present such a construction in this section, based on the work of Alain Connes, Niranjan Ramachandran, and the author [38].

We then discuss some ideas about the unsolved problem of real quadratic fields, based on Manin's real multiplication program [75] $[76]$. 
5.1. Imaginary quadratic fields and $\mathbb{K}$-lattices. Let $\mathcal{O}=\mathbb{Z}+$ $\mathbb{Z} \tau$ be the ring of integers of an imaginary quadratic field $\mathbb{K}=\mathbb{Q}(\tau)$; fix the imbedding $\mathbb{K} \hookrightarrow \mathbb{C}$ with $\tau \in \mathbb{H}$. We let $N: \mathbb{K}^{*} \rightarrow \mathbb{Q}^{*}$ be the norm map. This satisfies $N(x)=\operatorname{det}(g)$, for $g=q_{\tau}(x)$ the image under the embedding

$$
q_{\tau}: \mathbb{K}^{*} \hookrightarrow \mathrm{GL}_{2}(\mathbb{Q}),
$$

determined by the choice of $\tau$. The image of the embedding (3.62) is characterized by the property

$$
q_{\tau}\left(K^{*}\right)=\left\{g \in \mathrm{GL}_{2}^{+}(\mathbb{Q}): g(\tau)=\tau\right\} .
$$

A quantum statistical mechanical system for imaginary quadratic fields can be constructed by considering commensurability classes of 1-dimensional $\mathbb{K}$-lattices.

DEFINITION 5.1. For $\mathbb{K}$ an imaginary quadratic field, a 1-dimensional $\mathbb{K}$-lattice $(\Lambda, \phi)$ is a finitely generated $\mathcal{O}$-submodule $\Lambda \subset \mathbb{C}$, such that $\Lambda \otimes_{\mathcal{O}} \mathbb{K} \cong \mathbb{K}$, together with a morphism of $\mathcal{O}$-modules

$$
\phi: \mathbb{K} / \mathcal{O} \rightarrow \mathbb{K} \Lambda / \Lambda \text {. }
$$

A 1-dimensional $\mathbb{K}$-lattice is invertible if $\phi$ is an isomorphism of $\mathcal{O}$ modules.

A 1-dimensional $K$-lattice is, in particular, a 2-dimensional $\mathbb{Q}$ lattice. This plays an important role in the notion of commensurability.

Definition 5.2. Two 1-dimensional $\mathbb{K}$-lattices $\left(\Lambda_{1}, \phi_{1}\right)$ and $\left(\Lambda_{2}, \phi_{2}\right)$ are commensurable if $\mathbb{K} \Lambda_{1}=\mathbb{K} \Lambda_{2}$ and $\phi_{1}=\phi_{2}$ modulo $\Lambda_{1}+\Lambda_{2}$.

It turns out that two 1-dimensional $\mathbb{K}$-lattices are commensurable iff the underlying $\mathbb{Q}$-lattices are commensurable.

We can give a more explicit description of the data of 1-dimensional $\mathbb{K}$-lattices. Namely, the data $(\Lambda, \phi)$ of a 1 -dimensional $\mathbb{K}$-lattice are equivalent to data $(\rho, s)$ of an element $\rho \in \hat{\mathcal{O}}=\hat{\mathbb{Z}} \otimes \mathcal{O}$ and $s \in \mathbb{A}_{\mathbb{K}}^{*} / \mathbb{K}^{*}$, modulo the action of $\hat{\mathcal{O}}^{*}$ given by $(\rho, s) \mapsto\left(x^{-1} \rho, x s\right)$. Thus, the space of 1-dimensional $\mathbb{K}$-lattices is given by

$$
\hat{\mathcal{O}} \times_{\hat{\mathcal{O}}^{*}}\left(\mathbb{A}_{\mathbb{K}}^{*} / \mathbb{K}^{*}\right)
$$

Let $\mathbb{A}_{\mathbb{K}}=\mathbb{A}_{\mathbb{K}, f} \times \mathbb{C}^{*}$ be the subset of adèles of $\mathbb{K}$ with nontrivial archimedean component. There is an identification of the set of commensurability classes of 1 -dimensional $\mathbb{K}$-lattices (not up to scale) with the space $\mathbb{A}_{\mathbb{K}} / \mathbb{K}^{*}$. In turn, the set of commensurability classes of 1-dimensional $\mathbb{K}$-lattices up to scaling can be identified with the quotient

$$
\hat{\mathcal{O}} / \mathbb{K}^{*}=\mathbb{A}_{\mathbb{K}, f} / \mathbb{K}^{*}
$$


In the left hand side, the notation $\hat{\mathcal{O}} / \mathbb{K}^{*}$ stands for the equivalence classes of elements in $\hat{\mathcal{O}}$ modulo the equivalence relation given by the partially defined action of $\mathbb{K}^{*}$.

One can then introduce noncommutative algebras of coordinates associated to these quotient spaces. The procedure is analogous to the one followed in the $\mathrm{GL}_{2}$ and in the Bost-Connes case. If $\mathcal{L}_{\mathbb{K}}$ denotes the set of commensurability classes of 1-dimensional $\mathbb{K}$-lattices described above, then one considers the convolution algebra $C^{*}\left(\mathcal{L}_{\mathbb{K}}\right)$ given by the groupoid algebra of the commensurability relation. We regard the elements of this algebra as functions of pairs $f\left((\Lambda, \phi),\left(\Lambda^{\prime}, \phi^{\prime}\right)\right)$ with the convolution product dictated by the equivalence relation. The groupoid is by construction a subgroupoid of the the groupoid of commensurability classes of 2-dimensional $\mathbb{Q}$-lattices. When taking the quotient by scaling $\mathcal{L}_{\mathbb{K}} / \mathbb{C}^{*}$, we consider the convolution algebra $\mathcal{A}_{\mathbb{K}}:=C^{*}\left(\mathcal{L}_{\mathbb{K}} / \mathbb{C}^{*}\right)$, which in this case is still a groupoid algebra (unlike what happens in the $\mathrm{GL}_{2}$ case, $c f$. [38]). The algebra $\mathcal{A}_{\mathbb{K}}$ is unital as in the case of the Bost-Connes system.

The time evolution of the $\mathrm{GL}_{2}$ system induces a natural time evolution on $\mathcal{A}_{\mathbb{K}}$ given by

$$
\sigma_{t}(f)=N^{i t} f
$$

where $N: \mathbb{K}^{*} \rightarrow \mathbb{Q}^{*}$ is the norm map.

This quantum statistical mechanical system $\left(\mathcal{A}_{\mathbb{K}}, \sigma_{t}\right)$ has properties that are, in a sense, intermediate between the BC system and the $\mathrm{GL}_{2}-$ system.

Again one finds that invertible 1-dimensional $\mathbb{K}$-lattices $(\Lambda, \phi)$ give rise to positive energy representations of $\mathcal{A}_{\mathbb{K}}$ on the Hilbert space $\ell^{2}\left(G_{L}\right)$, where $G_{L}$ is the set of elements in the groupoid $\mathcal{L}_{\mathbb{K}} / \mathbb{C}^{*}$ with source $L=(\Lambda, \phi)$. The set of invertible 1-dimensional $\mathbb{K}$-lattices up to scale can be identified with the idèle class group

$$
C_{\mathbb{K}} / D_{\mathbb{K}}=\mathbb{A}_{\mathbb{K}, f}^{*} / \mathbb{K}^{*}
$$

For an invertible $\mathbb{K}$-lattice, one can identify the set $G_{L}$ above with the set of ideals $J \subset \mathcal{O}$. The Hamiltonian implementing the time evolution is then of the form

$$
H \epsilon_{J}=\log \mathfrak{n}(J) \epsilon_{J} .
$$

Thus, the partition function of the system $\left(\mathcal{A}_{\mathbb{K}}, \sigma_{t}\right)$ is the Dedekind zeta function $\zeta_{K}(\beta)$ of $\mathbb{K}$,

$$
Z(\beta)=\sum_{J \text { ideal in } \mathcal{O}} \mathfrak{n}(J)^{-\beta}=\zeta_{K}(\beta) .
$$


This is, as expected, the natural generalization of the Riemann zeta function $\zeta(\beta)=\zeta_{\mathbb{Q}}(\beta)$ to other number fields.

The symmetry group of the system is the group of idèle classes $\mathbb{A}_{\mathbb{K}, f}^{*}$, with the subgroup $\mathbb{K}^{*}$ acting by inner endomorphisms, so that the induced action on KMS states is by the idèle class group (3.68). As in the case of the $\mathrm{GL}_{2}$-system, this group of symmetries includes an action by endomorphisms. Only the subgroup $\hat{\mathcal{O}} / \mathcal{O}^{*}$, with $\mathcal{O}^{*}$ the group of units of $\mathcal{O}$, acts by automorphisms, while the full action of $\mathbb{A}_{\mathbb{K}, f}^{*} / \mathbb{K}^{*}$ also involves endomorphisms. This, in particular, shows the appearance of the class number of $\mathbb{K}$, as one can see from the commutative diagram

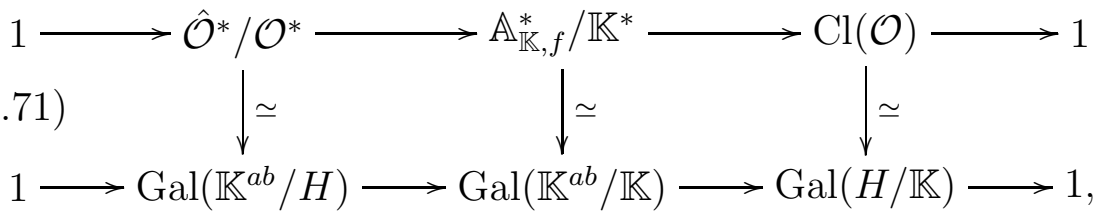

where $H$ is the Hilbert class field of $\mathbb{K}$, i.e. , its maximal abelian unramified extension. The ideal class group $\mathrm{Cl}(\mathcal{O})$ is naturally isomorphic to the Galois group $\mathrm{Gal}(H / \mathbb{K})$. The case of class number one is analogous to the BC system, as was already observed ( $c f .[60]$ ).

The arithmetic algebra $\mathcal{A}_{2, \mathbb{Q}}$ for the $\mathrm{GL}_{2}$-system induces, by restriction to the subgroupoid, a natural choice of a rational subalgebra $\mathcal{A}_{\mathbb{K}, \mathbb{Q}}$ of the system $\left(\mathcal{A}_{\mathbb{K}}, \sigma_{t}\right)$ for the imaginary quadratic field $\mathbb{K}$.

Notice that, because of the fact that the variable $z \in \mathbb{H}$ in the coordinates of the $\mathrm{GL}_{2}$-system is now only affecting finitely many values indexed by the elements of the class group $\mathrm{Cl}(\mathcal{O})$, we obtain that $\mathcal{A}_{\mathbb{K}, \mathbb{Q}}$ is a subalgebra of $\mathcal{A}_{\mathbb{K}}$ as in the $\mathrm{BC}$ case (though not involutive) and not just an algebra of unbounded multipliers as in the $\mathrm{GL}_{2}$-case.

With this choice of the arithmetic subalgebra, one obtains a result analogous to Theorem 4.3, see [38]:

THEOREM 5.1. The system $\left(\mathcal{A}_{\mathbb{K}}, \sigma_{t}\right)$ has the following properties.

(1) In the range $0<\beta \leq 1$ there is a unique KMS state.

(2) For $\beta>1$, extremal $K M S_{\beta}$ states are parameterized by invertible $\mathbb{K}$-lattices,

$$
\mathcal{E}_{\beta} \simeq \mathbb{A}_{\mathbb{K}, f}^{*} / \mathbb{K}^{*}
$$

with a free and transitive action of the idèle class group of $\mathbb{K}$ as symmetries.

(3) The set of extremal $K M S_{\infty}$ states (as weak limits of $K M S_{\beta}$ states) is still given by (3.72). The extremal $K M S_{\infty}$ states $\varphi_{\infty, L}$, evaluated on the arithmetic subalgebra $\mathcal{A}_{\mathbb{K}, \mathbb{Q}}$, generate 
the maximal abelian extension $\mathbb{K}^{a b}$. The class field theory isomorphism intertwines the action of $\mathbb{A}_{\mathbb{K}, f}^{*} / \mathbb{K}^{*}$ by symmetries of the system with the Galois action of $\mathrm{Gal}\left(\mathbb{K}^{a b} / \mathbb{K}\right)$ on the image of $\mathcal{A}_{\mathbb{K}, \mathbb{Q}}$ under the extremal $K M S_{\infty}$ states,

$$
\alpha \circ \varphi_{\infty, L}=\varphi_{\infty, L} \circ \theta(\alpha), \quad \forall \alpha \in \operatorname{Gal}\left(\mathbb{K}^{a b} / \mathbb{K}\right),
$$

where $\theta$ is the class field theory isomorphism

$$
\theta: \operatorname{Gal}\left(K^{a b} / K\right) \rightarrow \mathbb{A}_{K, f}^{*} / \mathbb{K}^{*}
$$

Now the classes of 1-dimensional $\mathbb{K}$ lattices, for $\mathbb{K}=\mathbb{Q}(\tau)$, viewed as 2-dimensional $\mathbb{Q}$-lattices $L=(\rho, z)$ correspond to only finitely many values of $z \in \mathbb{H} \cap \mathbb{K}$. Moreover, these points no longer satisfy the "generic" condition of Theorem 4.3. In fact, for a CM point $z \in \mathbb{H} \cap \mathbb{K}$, evaluation of elements $f$ in the modular field at $z$ no longer gives an embedding. The image $F_{\tau} \subset \mathbb{C}$ is in this case a copy of the maximal abelian extension of $\mathbb{K}(c f .[\mathbf{1 0 4}])$

$$
F \rightarrow F_{\tau}=\mathbb{K}^{a b} \subset \mathbb{C} .
$$

The explicit action of the Galois group $\operatorname{Gal}\left(\mathbb{K}^{a b} / \mathbb{K}\right)$ is obtained through the action of automorphisms of the modular field via Shimura reciprocity [104], as described in the following diagram with exact rows:

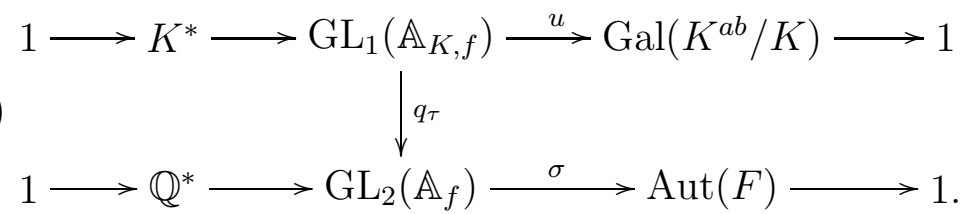

Here the map $u$ induces a quotient map $\bar{u}: \mathbb{A}_{K, f}^{*} / \mathbb{K}^{*} \rightarrow \operatorname{Gal}\left(K^{a b} / K\right)$ that is the inverse of the class field theory isomorphism (3.74), while $q_{\tau}$ is the embedding determined by the choice of $\tau \in H$ with $\mathbb{K}=\mathbb{Q}(\tau)$. Thus, the explicit Galois action is given by

$$
u(\gamma)(f(\tau))=f^{\sigma\left(q_{\tau}(\gamma)\right)}(\tau),
$$

where $f \mapsto f^{\alpha}$ denotes the action of $\alpha \in \operatorname{Aut}(F)$.

This provides the intertwining between the symmetries of the quantum statistical mechanical system and the Galois action on the image of states on the elements of the arithmetic subalgebra.

The structure of KMS states at positive temperatures is similar to the Bost-Connes case ( $c f$. [38] for detailed proofs). 
5.2. Real multiplication. The next fundamental question in the direction of generalizations of the BC system to other number fields is how to approach the more difficult case of real quadratic fields. We give a brief outline of Manin's ideas on real multiplication [75] [76], and suggest how they may combine with the $\mathrm{GL}_{2}$-system described above.

Recently, Manin developed a theory of real multiplication for noncommutative tori [76], aimed at providing a setting, within noncommutative geometry, in which to treat the problem of abelian extensions of real quadratic fields on a somewhat similar footing as the known case of imaginary quadratic fields, for which the theory of elliptic curves with complex multiplication provides the right geometric setup.

The first entry in the dictionary developed in [76] between elliptic curves with complex multiplication and noncommutative tori with real multiplication consists of a parallel between lattices and pseudolattices in $\mathbb{C}$,

$$
(\mathbb{C} \text {, lattice } \Lambda) \quad \rightsquigarrow(\mathbb{R} \text {, pseudo-lattice } L) \text {. }
$$

By a pseudo-lattice one means the data of a free abelian group of rank two, with an injective homomorphism to a 1-dimensional complex vector space, such that the image lies in an oriented real line.

This aims at generalizing the well known equivalence between the category of elliptic curves and the category of lattices, realized by the period functor, to a setting that includes noncommutative tori.

As we have seen previously, any 2-dimensional lattice is, up to isomorphism, of the form $\Lambda_{\tau}=\mathbb{Z}+\mathbb{Z} \tau$, for $\tau \in \mathbb{C} \backslash \mathbb{R}$ and non-trivial morphisms between such lattices are given by the action of matrices $M_{2}(\mathbb{Z})$ by fractional linear transformations. Thus, the moduli space of lattices up to isomorphism is given by the quotient of $\mathbb{P}^{1}(\mathbb{C}) \backslash \mathbb{P}^{1}(\mathbb{R})$ by $\mathrm{PGL}_{2}(\mathbb{Z})$.

A pseudo-lattice is, up to isomorphism, of the form $L_{\theta}=\mathbb{Z}+\mathbb{Z} \theta$, for $\theta \in \mathbb{R} \backslash \mathbb{Q}$, and non-trivial morphisms of pseudo-lattices are again given by matrices in $M_{2}(\mathbb{Z})$ acting by fractional linear transformations on $\mathbb{P}^{1}(\mathbb{R})$. The moduli space of pseudo-lattices is given by the quotient of $\mathbb{P}^{1}(\mathbb{R})$ by the action of $\operatorname{PGL}(2, \mathbb{Z})$. Since the action does not give rise to a nice classical quotient, this moduli space should be treated as a noncommutative space.

As described at length in the previous chapter, the resulting space represents a component in the "boundary" of the classical moduli space of elliptic curves, which parameterizes those degenerations from lattices to pseudo-lattices, which are invisible to the usual algebro-geometric setting. 
The cusp, i.e. the orbit of $\mathbb{P}^{1}(\mathbb{Q})$, corresponds to the degenerate case where the image in $\mathbb{C}$ of the rank two free abelian group has rank one.

This gives another entry in the dictionary, regarding the moduli spaces:

$$
\mathbb{H} / \operatorname{PSL}(2, \mathbb{Z}) \quad \leadsto \quad C\left(\mathbb{P}^{1}(\mathbb{R})\right) \rtimes \operatorname{PGL}(2, \mathbb{Z}) .
$$

In the correspondence of pseudo-lattices and noncommutative tori, the group of invertible morphisms of pseudo-lattices corresponds to isomorphisms of noncommutative tori realized by strong Morita equivalences. In this context a "morphism" is not given as a morphism of algebras but as a map of the category of modules, obtained by tensoring with a bimodule. The notion of Morita equivalences as morphisms fits into the more general context of correspondences for operator algebras as in $[\mathbf{2 1}] \S \mathrm{V} . \mathrm{B}$, as well as in the algebraic approach to noncommutative spaces of [102].

The category of noncommutative tori is defined by considering as morphisms the isomorphism classes of $\left(C^{*}-\right)$ bimodules that are the ranges of projections. The functor from noncommutative tori to pseudolattices ( $c f .[75] \S 3.3$ and [76] $\S 1.4)$ is then given on objects by

$$
\mathcal{T}_{\theta}^{2} \mapsto\left(K_{0}\left(\mathcal{A}_{\theta}\right), H C_{0}\left(\mathcal{A}_{\theta}\right), \tau: K_{0}\left(\mathcal{A}_{\theta}\right) \rightarrow H C_{0}\left(\mathcal{A}_{\theta}\right)\right) .
$$

Here $\mathcal{A}_{\theta}$ is the algebra of the noncommutative torus, $H C_{0}\left(\mathcal{A}_{\theta}\right)=$ $\mathcal{A}_{\theta} /\left[\mathcal{A}_{\theta}, \mathcal{A}_{\theta}\right]$, with $\tau$ the universal trace, and the orientation is determined by the cone of positive elements in $K_{0}$. On morphisms the functor is given by

$$
\mathcal{M}_{\theta, \theta^{\prime}} \mapsto\left([\mathcal{E}] \mapsto\left[\mathcal{E} \otimes_{\mathcal{A}_{\theta}} \mathcal{M}_{\theta, \theta^{\prime}}\right]\right),
$$

where $\mathcal{M}_{\theta, \theta^{\prime}}$ are the bimodules constructed by Connes in [16]. A crucial point in this definition is the fact that, for noncommutative tori, finite projective modules are classified by the value of a unique normalized trace $(c f .[\mathbf{1 6}][\mathbf{1 0 1}])$.

The functor of (3.77) (3.78) is weaker than an equivalence of categories. For instance, it maps trivially all ring homomorphisms that act trivially on $K_{0}$. However, this correspondence is sufficient to develop a theory of real multiplication for noncommutative tori parallel to the theory of complex multiplication for elliptic curves $(c f .[75][\mathbf{7 6}])$.

For lattices/elliptic curves, the typical situation is that $\operatorname{End}(\Lambda)=\mathbb{Z}$, but there are exceptional lattices for which $\operatorname{End}(\Lambda) \supsetneq \mathbb{Z}$. In this case, there exists a complex quadratic field $\mathbb{K}$ such that $\Lambda$ is isomorphic to a lattice in $\mathbb{K}$. More precisely, the endomorphism ring is given by $\operatorname{End}(\Lambda)=\mathbb{Z}+f \mathcal{O}$, where $\mathcal{O}$ is the ring of integers of $\mathbb{K}$ and the integer $f \geq 1$ is called the conductor. Such lattices are said to have complex 
multiplication. The elliptic curve $E_{\mathbb{K}}$ with $E_{\mathbb{K}}(\mathbb{C})=\mathbb{C} / \mathcal{O}$ is endowed with a complex multiplication map, given on the universal cover by $x \mapsto a x, a \in \mathcal{O}$.

Similarly, there is a parallel situation for pseudo-lattices: $\operatorname{End}(L) \supsetneq$ $\mathbb{Z}$ happens when there exists a real quadratic field $\mathbb{K}$ such that $L$ is isomorphic to a pseudolattice contained in $\mathbb{K}$. In this case, one also has $\operatorname{End}(L)=\mathbb{Z}+f \mathcal{O}$. Such pseudo-lattices are said to have real multiplication. The pseudo-lattices $L_{\theta}$ that have real multiplication correspond to values of $\theta \in \mathbb{R} \backslash \mathbb{Q}$ that are quadratic irrationalities. These are characterized by having eventually periodic continued fraction expansion. The "real multiplication map" is given by tensoring with a bimodule: in the case of $\theta$ with periodic continued fraction expansion, there is an element $g \in \operatorname{PGL}(2, \mathbb{Z})$ such that $g \theta=\theta$, to which we can associate an $\mathcal{A}_{\theta}-\mathcal{A}_{\theta}$ bimodule $\mathcal{E}$.

An analog of isogenies for noncommutative tori is obtained by considering morphisms of pseudolattices $L_{\theta} \mapsto L_{n \theta}$ which correspond to a Morita morphism given by $\mathcal{A}_{\theta}$ viewed as an $\mathcal{A}_{n \theta}-\mathcal{A}_{\theta}$ bimodule, where $\mathcal{A}_{n \theta} \hookrightarrow \mathcal{A}_{\theta}$ by $U \mapsto U^{n}, V \mapsto V$.

By considering isogenies, one can enrich the dictionary between the moduli space of elliptic curves and the moduli space of Morita equivalent noncommutative tori. This leads one to consider the whole tower of modular curves parameterizing elliptic curves with level structure and the corresponding tower of noncommutative modular curves described in the previous chapter $(c f .[83])$,

$$
\mathbb{H} / G \leftrightarrow C\left(\mathbb{P}^{1}(\mathbb{R}) \times \mathbb{P}\right) \rtimes \Gamma,
$$

for $\Gamma=\operatorname{PGL}(2, \mathbb{Z})$ (or $\operatorname{PSL}(2, \mathbb{Z}))$ and $G \subset \Gamma$ a finite index subgroup, with $\mathbb{P}=G \backslash \Gamma$ the coset space.

In the problem of constructing the maximal abelian extension of complex quadratic fields, one method is based on evaluating at the torsion points of the elliptic curve $E_{\mathbb{K}}$ a power of the Weierstrass function. This means considering the corresponding values of a coordinate on the projective line $E_{\mathbb{K}} / \mathcal{O}^{*}$. The analogous object in the noncommutative setting, replacing this projective line, should be $(c f .[76])$ a crossed product of functions on $\mathbb{K}$ by the $a x+b$ group with $a \in \mathcal{O}^{*}$ and $b \in \mathcal{O}$, for a real quadratic field $\mathbb{K}$.

A different method to construct abelian extensions of a complex quadratic field $\mathbb{K}$ is via Stark numbers. Following the notation of $[\mathbf{7 6}]$, if $j: \Lambda \rightarrow V$ is an injective homomorphism of a free abelian group of rank two to a one-dimensional complex vector space, and $\lambda_{0} \in \Lambda \otimes \mathbb{Q}$, 
then one can consider the zeta function

$$
\zeta\left(\Lambda, \lambda_{0}, s\right)=\sum_{\lambda \in \Lambda} \frac{1}{\left|j\left(\lambda_{0}+\lambda\right)\right|^{2 s}} .
$$

Stark proved $(c f .[\mathbf{1 0 5}])$ that the numbers

$$
S\left(\Lambda, \lambda_{0}\right)=\exp \zeta^{\prime}\left(\Lambda, \lambda_{0}, 0\right)
$$

are algebraic units generating certain abelian extensions of $\mathbb{K}$. The argument in this case is based on a direct computational tool (the Kronecker second limit formula) and upon reducing the problem to the theory of complex multiplication. There is no known independent argument for the Stark conjectures, while the analogous question is open for the case of real quadratic fields.

For a real quadratic field $\mathbb{K}$, instead of zeta functions of the form (3.80), the conjectural Stark units are obtained from zeta functions of the form (in the notation of $[\mathbf{7 6}]$ )

$$
\zeta\left(L, l_{0}, s\right)=\operatorname{sgn} l_{0}^{\prime} N(\mathfrak{a})^{s} \sum \frac{\operatorname{sgn}\left(l_{0}+l\right)^{\prime}}{\left|N\left(l_{0}+l\right)\right|^{s}},
$$

where $l \mapsto l^{\prime}$ is the action of the nontrivial element in $\operatorname{Gal}(\mathbb{K} / \mathbb{Q})$ and $N(l)=l l^{\prime}$. The element $l_{0} \in O$ is chosen so that the ideals $\mathfrak{a}=\left(L, l_{0}\right)$ and $\left(l_{0}\right) \mathfrak{a}^{-1}$ are coprime with $L \mathfrak{a}^{-1}$, and the summation over $l \in L$ in (3.82) is restricted by taking only one representative from each coset class $\left(l_{0}+l\right) \epsilon$, for units $\epsilon$ satisfying $\left(l_{0}+L\right) \epsilon=\left(l_{0}+L\right)$, i.e. $\epsilon \equiv 1$ $\bmod L \mathfrak{a}^{-1}$. Then the Stark numbers are given as in (3.81) by (cf. [76])

$$
S\left(L, l_{0}\right)=\exp \zeta^{\prime}\left(L, l_{0}, 0\right) .
$$

In [76], Manin develops an approach to the computation of sums (3.82) based on a version of theta functions for pseudo-lattices, which are obtained by averaging theta constants of complex lattices along geodesics with ends at a pair of conjugate quadratic irrationalities $\theta, \theta^{\prime}$ in $\mathbb{R} \backslash \mathbb{Q}$.

This procedure fits into a general philosophy, according to which one can recast part of the arithmetic theory of modular curves in terms of the noncommutative boundary (3.79) by studying the limiting behavior when $\tau \rightarrow \theta \in \mathbb{R} \backslash \mathbb{Q}$ along geodesics, or some averaging along such geodesics. In general, a nontrivial result is obtained when approaching $\theta$ along a path that corresponds to a geodesic in the modular curve that spans a limiting cycle, which is the case precisely when the endpoint $\theta$ is a quadratic irrationality. An example of this type of behavior is the theory of "limiting modular symbols" developed in [83], [84]. 
5.3. Pseudolattices and the $\mathrm{GL}_{2}$-system. The noncommutative space (3.35) of the $\mathrm{GL}_{2}$-system also admits a compactification, now given by adding the boundary $\mathbb{P}^{1}(\mathbb{R})$ to $\mathbb{H}^{ \pm}$, as in the noncommutative compactification of modular curves of $[83]$,

$$
\begin{aligned}
\overline{S h^{(n c)}}\left(\mathrm{GL}_{2}, \mathbb{H}^{ \pm}\right):= & \mathrm{GL}_{2}(\mathbb{Q}) \backslash\left(M_{2}\left(\mathbb{A}_{f}\right) \times \mathbb{P}^{1}(\mathbb{C})\right) \\
& =\mathrm{GL}_{2}(\mathbb{Q}) \backslash M_{2}(\mathbb{A}) / \mathbb{C}^{*},
\end{aligned}
$$

where $\mathbb{P}^{1}(\mathbb{C})=\mathbb{H}^{ \pm} \cup \mathbb{P}^{1}(\mathbb{R})$.

This corresponds to adding to the space of commensurability classes of 2-dimensional $\mathbb{Q}$-lattices the pseudolattices in the sense of $[\mathbf{7 6}]$, here considered together with a $\mathbb{Q}$-structure. It seems then that Manin's real multiplication program may fit in with the boundary of the noncommutative space of the $\mathrm{GL}_{2}$-system. The crucial question in this respect becomes the construction of an arithmetic algebra associated to the noncommutative modular curves. The results illustrated in the previous chapter, regarding identities involving modular forms at the boundary of the classical modular curves and limiting modular symbols, as well as the still mysterious phenomenon of "quantum modular forms" identified by Zagier, point to the fact that there should exist a rich class of objects replacing modular forms, when "pushed to the boundary".

Regarding the role of modular forms, notice that, in the case of (3.84), we can again consider the dual system. This is a $\mathbb{C}^{*}$-bundle

$$
\mathcal{L}_{2}=\mathrm{GL}_{2}(\mathbb{Q}) \backslash M_{2}(\mathbb{A}) .
$$

On this dual space modular forms appear naturally instead of modular functions and the algebra of coordinates contains the modular Hecke algebra of Connes-Moscovici ([41], [42]) as arithmetic subalgebra.

Thus, the noncommutative geometry of the space of $\mathbb{Q}$-lattices modulo the equivalence relation of commensurability provides a setting that unifies several phenomena involving the interaction of noncommutative geometry and number theory. These include the Bost-Connes system, the noncommutative space underlying the construction of the spectral realization of the zeros of the Riemann zeta function in [23], the modular Hecke algebras of [41] [42], and the noncommutative modular curves of $[83]$. 



\section{CHAPTER 4}

\section{Noncommutative geometry at arithmetic infinity}

This chapter is based on joint work of Katia Consani and the author $([44],[45],[46],[47],[48])$ that proposes a model for the dual graph of the maximally degenerate fibers at the archimedean places of an arithmetic surface in terms of a noncommutative space (spectral triple) related to the action of a Schottky group on its limit set. This description of $\infty$-adic geometry provides a compatible setting that combines Manin's result [79] on the Arakelov Green function for arithmetic surfaces in terms of hyperbolic geometry and for a cohomological construction of Consani [43] associated to the archimedean fibers of arithmetic varieties, related to Deninger's calculation of the local $L$-factors as regularized determinants ( $c f$. [52], [53]).

\section{Schottky uniformization}

Topologically a compact Riemann surface $X$ of genus $g$ is obtained by gluing the sides of a $4 g$-gon. Correspondingly, the fundamental group has a presentation

$$
\pi_{1}(X)=\left\langle a_{1}, \ldots, a_{g}, b_{1}, \ldots, b_{g} \mid \prod_{i}\left[a_{i}, b_{i}\right]=1\right\rangle,
$$

where the generators $a_{i}$ and $b_{i}$ label the sides of the polygon.

In the genus $g=1$ case, the parallelogram is the fundamental domain of the $\pi_{1}(X) \simeq \mathbb{Z}^{2}$ action on the plane $\mathbb{C}$, so that $X=\mathbb{C} /(\mathbb{Z}+\mathbb{Z} \tau)$ is an elliptic curve.

For genus at least $g \geq 2$, the hyperbolic plane $\mathbb{H}^{2}$ admits a tessellation by regular $4 g$-gons, and the action of the fundamental group by deck transformations is realized by the action of a subgroup $\pi_{1}(X) \simeq$ $G \subset \operatorname{PSL}(2, \mathbb{R})$ by isometries of $\mathbb{H}^{2}$. This endows the compact Riemann surface $X$ with a hyperbolic metric and a Fuchsian uniformization

$$
X=G \backslash \mathbb{H}^{2} .
$$

Another, less well known, type of uniformization of compact Riemann surfaces is Schottky uniformization. We recall briefly some general facts on Schottky groups. 


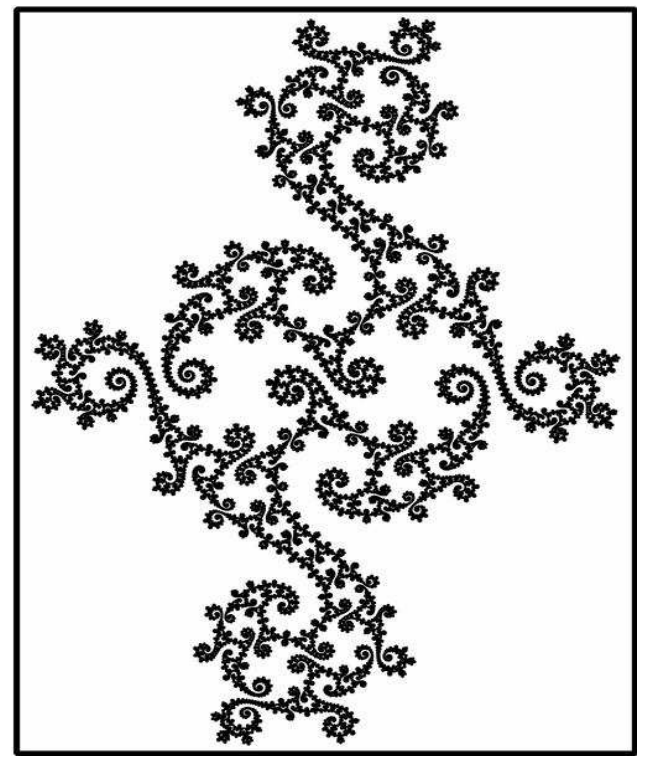

Figure 1. Limit set of a Schottky group

1.1. Schottky groups. A Schottky group of rank $g$ is a discrete subgroup $\Gamma \subset \operatorname{PSL}(2, \mathbb{C})$, which is purely loxodromic and isomorphic to a free group of rank $g$. The group $\operatorname{PSL}(2, \mathbb{C})$ acts on $\mathbb{P}^{1}(\mathbb{C})$ by fractional linear transformations

$$
\gamma: z \mapsto \frac{a z+b}{c z+d}
$$

where $\mathbb{P}^{1}(\mathbb{C})$ is viewed as the sphere at infinity of the three dimensional real hyperbolic space $\mathbb{H}^{3}$ and $\operatorname{PSL}(2, \mathbb{C})$ is the group of isometries of $\mathbb{H}^{3}$. Recall that an element $\gamma \in \operatorname{PSL}(2, \mathbb{C}), \gamma \neq 1$, is parabolic, loxodromic, or elliptic, respectively, if it has one, two, or infinitely many fixed points in $\mathbb{H}^{3} \cup \mathbb{P}^{1}(\mathbb{C})$. Only in the elliptic case there are fixed points inside $\mathbb{H}^{3}$. In the other cases they lie on the sphere at infinity $\mathbb{P}^{1}(\mathbb{C})$.

For $\Gamma$ a Schottky group, we denote by $\Lambda_{\Gamma}$ the limit set of the action of $\Gamma$ on $\mathbb{H}^{3} \cup \mathbb{P}^{1}(\mathbb{C})$. This is the smallest non-empty closed $\Gamma$-invariant subset of $\mathbb{P}^{1}(\mathbb{C})$. This set can also be described as the closure of the set of the attractive and repelling fixed points $z^{ \pm}(\gamma)$ of the loxodromic elements $\gamma \in \Gamma$. In the case $g=1$ the limit set consists of two points, but for $g \geq 2$ the limit set is usually a fractal of some Hausdorff dimension $0 \leq \delta=\operatorname{dim}_{H}\left(\Lambda_{\Gamma}\right)<2$ (cf. e.g. Figure $\left.1^{1}\right)$.

\footnotetext{
${ }^{1}$ Figures 1 and 3 are taken from "Indra's pearls" by Mumford, Series, and Wright, [94]
} 

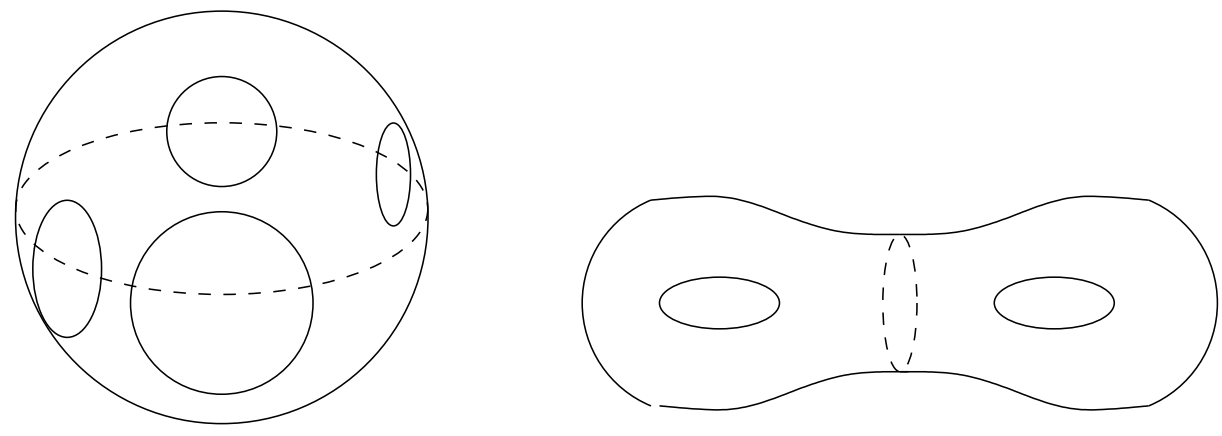

FiguRE 2. Schottky uniformization for $g=2$

We denote by $\Omega_{\Gamma}$ the domain of discontinuity of $\Gamma$, that is, the complement of $\Lambda_{\Gamma}$ in $\mathbb{P}^{1}(\mathbb{C})$. The quotient

$$
X(\mathbb{C})=\Gamma \backslash \Omega_{\Gamma}
$$

is a Riemann surface of genus $g$ and the covering $\Omega_{\Gamma} \rightarrow X(\mathbb{C})$ is called a Schottky uniformization of $X(\mathbb{C})$.

Every compact Riemann surface $X(\mathbb{C})$ admits a Schottky uniformization.

Let $\left\{\gamma_{i}\right\}_{i=1}^{g}$ be a set of generators of the Schottky group $\Gamma$. We write $\gamma_{i+g}=\gamma_{i}^{-1}$. There are $2 g$ Jordan curves $\gamma_{k}$ on the sphere at infinity $\mathbb{P}^{1}(\mathbb{C})$, with pairwise disjoint interiors $D_{k}$, such that the elements $\gamma_{k}$ are given by fractional linear transformations that map the interior of $C_{k}$ to the exterior of $C_{j}$ with $|k-j|=g$. The curves $C_{k}$ give a marking of the Schottky group. The markings are circles in the case of classical Schottky groups. A fundamental domain for the action of a classical Schottky group $\Gamma$ on $\mathbb{P}^{1}(\mathbb{C})$ is the region exterior to $2 g$ circles (cf. Figure 2).

1.2. Schottky and Fuchsian. Notice that, unlike Fuchsian uniformization, where the covering $\mathbb{H}^{2}$ is the universal cover, in the case of Schottky uniformization $\Omega_{\Gamma}$ is very far from being simply connected, in fact it is the complement of a Cantor set.

The relation between Fuchsian and Schottky uniformization is given by passing to the covering that corresponds to the normal subgroup $N\left\langle a_{1}, \ldots, a_{g}\right\rangle$ of $\pi_{1}(X)$ generated by half the generators $\left\{a_{1}, \ldots, a_{g}\right\}$,

$$
\Gamma \simeq \pi_{1}(X) / N\left\langle a_{1}, \ldots, a_{g}\right\rangle
$$



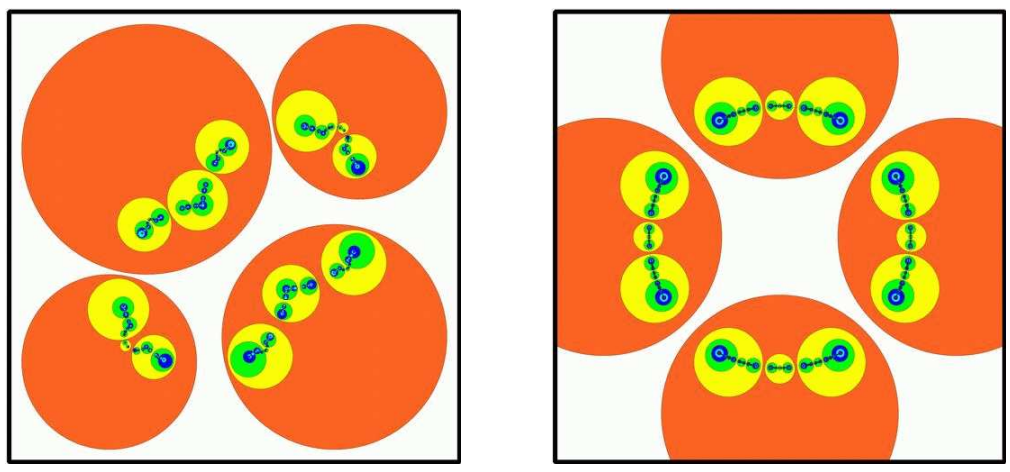

Figure 3. Classical and Fuchsian Schottky groups

with a corresponding covering map

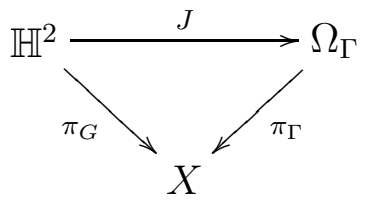

At the level of moduli, there is a corresponding map between the Teichmüller space $\mathcal{T}_{g}$ and the Schottky space $\mathcal{S}_{g}$, which depends on $3 g-3$ complex moduli.

\subsection{Surface with boundary: simultaneous uniformization.} To better visualize geometrically the Schottky uniformization of a compact Riemann surface, we can relate it to a simultaneous uniformization of the upper and lower half planes that yields two Riemann surfaces with boundary, joined at the boundary.

A Schottky group that is specified by real parameters so that it lies in $\operatorname{PSL}(2, \mathbb{R})$ is called a Fuchsian Schottky group (cf. Figure 3). Viewed as a group of isometries of the hyperbolic plane $\mathbb{H}^{2}$, or equivalently of the Poincaré disk, a Fuchsian Schottky group $G$ produces a quotient $G \backslash \mathbb{H}^{2}$, which is topologically a Riemann surface with boundary.

A quasi-circle for $\Gamma$ is a Jordan curve $C$ in $\mathbb{P}^{1}(\mathbb{C})$ which is invariant under the action of $\Gamma$. In particular, such a curve contains the limit set $\Lambda_{\Gamma}$. It was proved by Bowen that, if $X(\mathbb{C})$ is a Riemann surface of genus $g \geq 2$ with Schottky uniformization, then there exists always a quasi-circle for $\Gamma$.

We have then $\mathbb{P}^{1}(\mathbb{C}) \backslash C=\Omega_{1} \cup \Omega_{2}$ and, for $\pi_{\Gamma}: \Omega_{\Gamma} \rightarrow X(\mathbb{C})$ the covering map,

$$
\hat{C}=\pi_{\Gamma}\left(C \cap \Omega_{\Gamma}\right) \subset X(\mathbb{C})
$$



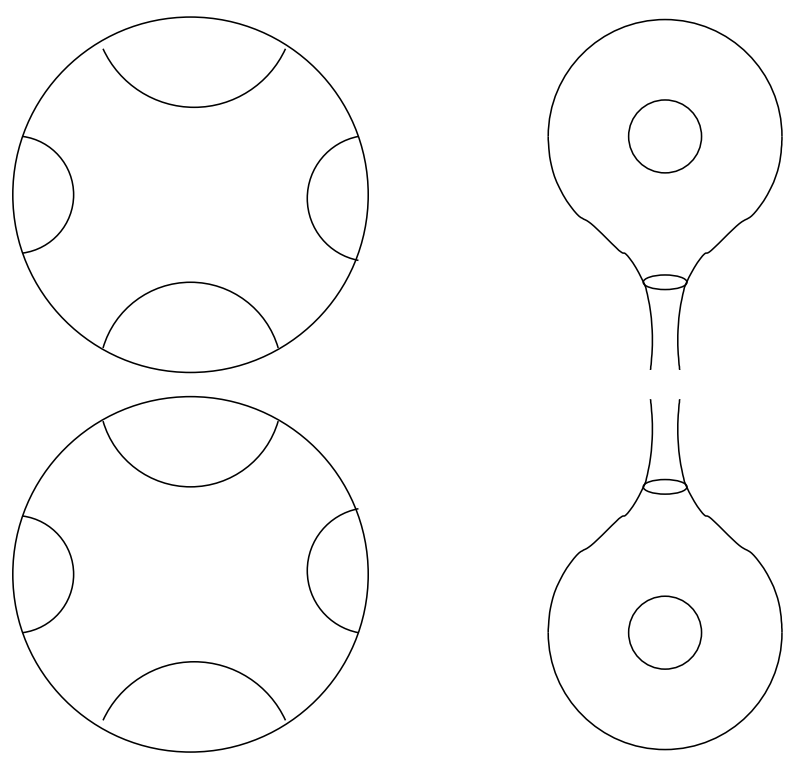

Figure 4. Fuchsian Schottky groups: Riemann surfaces with boundary

is a set of curves on $X(\mathbb{C})$ that disconnect the Riemann surface in the union of two surfaces with boundary, uniformized respectively by $\Omega_{1}$ and $\Omega_{2}$.

There exist conformal maps

$$
\alpha_{i}: \Omega_{i} \stackrel{\simeq}{\rightarrow} U_{i}, \quad U_{1} \cup U_{2}=\mathbb{P}^{1}(\mathbb{C}) \backslash \mathbb{P}^{1}(\mathbb{R})
$$

with $U_{i} \simeq \mathbb{H}^{2}=$ upper and lower half planes in $\mathbb{P}^{1}(\mathbb{C})$, and with

$$
G_{i}:=\left\{\alpha_{i} \gamma \alpha_{i}{ }^{-1}: \gamma \in \tilde{\Gamma}\right\} \simeq \Gamma
$$

Fuchsian Schottky groups $G_{i} \subset \operatorname{PSL}(2, \mathbb{R})$. Here $\tilde{\Gamma} \subset \operatorname{SL}(2, \mathbb{R})$ is the $\Gamma$-stabilizer of each of the two connected components in $\mathbb{P}^{1}(\mathbb{C}) \backslash C$.

The compact Riemann surface $X(\mathbb{C})$ is thus obtained as

$$
X(\mathbb{C})=X_{1} \cup_{\partial X_{1}=\hat{C}=\partial X_{2}} X_{2},
$$

with $X_{i}=U_{i} / G_{i}$ Riemann surfaces with boundary $\hat{C}$ (cf. Figure 4$)$.

In the case where $X(\mathbb{C})$ has a real structure $\iota: X \rightarrow X$, and the fixed point set $F i x(\iota)=X(\mathbb{R})$ of the involution $\iota$ is nonempty, we have in fact $\hat{C}=X(\mathbb{R})$, and the quasi-circle is given by $\mathbb{P}^{1}(\mathbb{R})$.

Notice that, in the case of a Fuchsian Schottky group, the Hausdorff dimension $\operatorname{dim}_{H} \Lambda_{\Gamma}$ of the limit set is in fact bounded above by 1 , since $\Lambda_{\Gamma}$ is contained in the rectifiable quasi-circle $\mathbb{P}^{1}(\mathbb{R})$. 


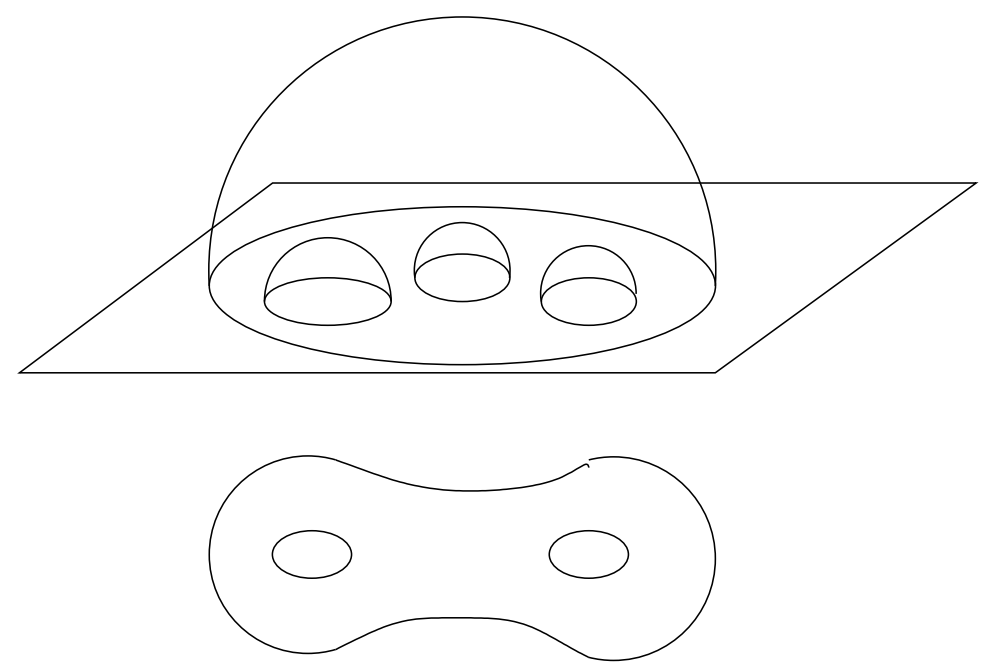

Figure 5. Genus two: fundamental domain in $\mathbb{H}^{3}$

1.4. Hyperbolic Handlebodies. The action of a rank $g$ Schottky group $\Gamma \subset \operatorname{PSL}(2, \mathbb{C})$ on $\mathbb{P}^{1}(\mathbb{C})$, by fractional linear transformations, extends to an action by isometries on real hyperbolic 3 -space $\mathbb{H}^{3}$. For a classical Schottky group, a fundamental domain in $\mathbb{H}^{3}$ is given by the region external to $2 g$ half spheres over the circles $C_{k} \subset \mathbb{P}^{1}(\mathbb{C})$ (cf. Figure 5).

The quotient

$$
\mathfrak{X}_{\Gamma}=\mathbb{H}^{3} / \Gamma
$$

is topologically a handlebody of genus $g$ filling the Riemann surface $X(\mathbb{C})$.

Metrically, $\mathfrak{X}_{\Gamma}$ is a real hyperbolic 3-manifold of infinite volume, having $X(\mathbb{C})$ as its conformal boundary at infinity $X(\mathbb{C})=\partial \mathfrak{X}_{\Gamma}$.

We denote by $\overline{\mathfrak{X}}_{\Gamma}$ the compactification obtained by adding the conformal boundary at infinity,

$$
\overline{\mathfrak{X}}_{\Gamma}=\left(\mathbb{H}^{3} \cup \Omega_{\Gamma}\right) / \Gamma .
$$

In the genus zero case, we just have the sphere $\mathbb{P}^{1}(\mathbb{C})$ as the conformal boundary at infinity of $\mathbb{H}^{3}$, thought of as the unit ball in the Poincaré model.

In the genus one case we have a solid torus $\mathbb{H}^{3} / q^{\mathbb{Z}}$, for $q \in \mathbb{C}^{*}$ acting as

$$
q(z, y)=(q z,|q| y)
$$

in the upper half space model, with conformal boundary at infinity the Jacobi uniformized elliptic curve $\mathbb{C}^{*} / q^{\mathbb{Z}}$. 


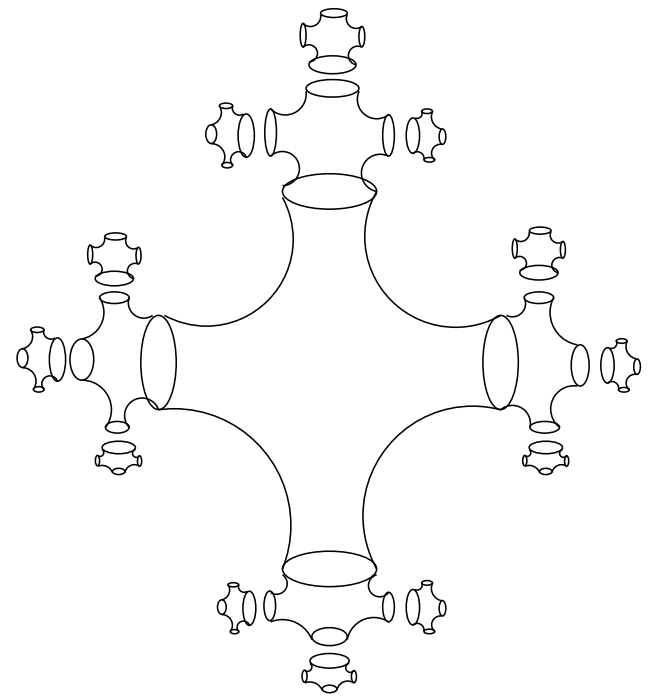

Figure 6. Handlebody of genus 2: fundamental domains in $\mathbb{H}^{3}$

In this case, the limit set consists of the points $\{0, \infty\}$, the domain of discontinuity is $\mathbb{C}^{*}$ and a fundamental domain is the annulus $\{|q|<$ $|z| \leq 1\}$ (exterior of two circles).

The relation of Schottky uniformization to the usual Euclidean uniformization of complex tori $X=\mathbb{C} /(\mathbb{Z}+\tau \mathbb{Z})$ is given by $q=\exp (2 \pi i \tau)$.

We shall return to the genus one case where we discuss a physical interpretation of Manin's result on the Green function. For our purposes, however, the most interesting case is when the genus is $g \geq 2$. In this case, the limit set $\Lambda_{\Gamma}$ is a Cantor set with an interesting dynamics of the action of $\Gamma$. It is the dynamics of the Schottky group on its limit set that generates an interesting noncommutative space.

1.5. Geodesics in $\mathfrak{X}_{\Gamma}$. The hyperbolic handlebody $\mathfrak{X}_{\Gamma}$ has infinite volume, but it contains a region of finite volume, which is a deformation retract of $\mathfrak{X}_{\Gamma}$. This is called the "convex core" of $\mathfrak{X}_{\Gamma}$ and is obtained by taking the geodesic hull of the limit set $\Lambda_{\Gamma}$ in $\mathbb{H}^{3}$ and then the quotient by $\Gamma$.

We identify different classes of infinite geodesics in $\mathfrak{X}_{\Gamma}$.

- Closed geodesics: since $\Gamma$ is purely loxodromic, for all $\gamma \in \Gamma$ there exist two fixed points $\left\{z^{ \pm}(\gamma)\right\} \in \mathbb{P}^{1}(\mathbb{C})$. The geodesics in $\mathbb{H}^{3} \cup \mathbb{P}^{1}(\mathbb{C})$ with ends at two such points $\left\{z^{ \pm}(\gamma)\right\}$, for some $\gamma \in \Gamma$, correspond to closed geodesics in the quotient $\mathfrak{X}_{\Gamma}$. 
- Bounded geodesics: The images in $\mathfrak{X}_{\Gamma}$ of geodesics in $\mathbb{H}^{3} \cup \mathbb{P}^{1}(\mathbb{C})$ having both ends on the limit set $\Lambda_{\Gamma}$ are geodesics that remain confined within the convex core of $\mathfrak{X}_{\Gamma}$.

- Unbounded geodesics: these are the geodesics in $\mathfrak{X}_{\Gamma}$ that eventually wander off the convex core towards the conformal boundary $X(\mathbb{C})$ at infinity. They correspond to geodesics in $\mathbb{H}^{3} \cup$ $\mathbb{P}^{1}(\mathbb{C})$ with at least one end at a point of $\Omega_{\Gamma}$.

In the genus one case, there is a unique primitive closed geodesic, namely the image in the quotient of the geodesic in $\mathbb{H}^{3}$ connecting 0 and $\infty$. The bounded geodesics are those corresponding to geodesics in $\mathbb{H}^{3}$ originating at 0 or $\infty$.

The most interesting case is that of genus $g \geq 2$, where the bounded geodesics form a complicated tangle inside $\mathfrak{X}_{\Gamma}$. Topologically, this is a generalized solenoid, namely it is locally the product of a line and a Cantor set.

\section{Dynamics and noncommutative geometry}

Since the uniformizing group $\Gamma$ is a free group, there is a simple way of obtaining a coding of the bounded geodesics in $\mathfrak{X}_{\Gamma}$. The set of such geodesics can be identified with $\Lambda_{\Gamma} \times_{\Gamma} \Lambda_{\Gamma}$, by specifying the endpoints in $\mathbb{H}^{3} \cup \mathbb{P}^{1}(\mathbb{C})$ modulo the action of $\Gamma$.

If $\left\{\gamma_{i}\right\}_{i=1}^{g}$ is a choice of generators of $\Gamma$ and $\gamma_{i+g}=\gamma_{i}^{-1}, i=1, \ldots, g$, we can introduce a subshift of finite type $(\mathcal{S}, T)$ where

$$
\mathcal{S}=\left\{\ldots a_{-m} \ldots a_{-1} a_{0} a_{1} \ldots a_{\ell} \ldots \mid a_{i} \in\left\{\gamma_{i}\right\}_{i=1}^{2 g}, a_{i+1} \neq a_{i}^{-1}, \forall i \in \mathbb{Z}\right\}
$$

is the set of doubly infinite words in the generators and their inverses, with the admissibility condition that no cancellations occur. The map $T$ is the invertible shift

$$
T\left(\ldots a_{-m} \ldots a_{-1} a_{0} a_{1} \ldots a_{\ell} \ldots\right)=\ldots a_{-m+1} \ldots a_{0} a_{1} a_{2} \ldots a_{\ell+1} \ldots
$$

Then we can pass from the discrete dynamical system $(\mathcal{S}, T)$ to its suspension flow and obtain the mapping torus

$$
\mathcal{S}_{T}:=\mathcal{S} \times[0,1] /(x, 0) \sim(T x, 1) .
$$

Topologically this space is a solenoid, that is, a bundle over $S^{1}$ with fiber a Cantor set.

2.1. Homotopy quotient. The space $\mathcal{S}_{T}$ of (4.6) has a natural interpretation in noncommutative geometry as the homotopy quotient 
in the sense of Baum-Connes [7] of the noncommutative space given by the $C^{*}$-algebra

$$
C(\mathcal{S}) \rtimes_{T} \mathbb{Z}
$$

describing the action of the shift (4.5) on the totally disconnected space (4.4). The noncommutative space (4.7) parameterizes bounded geodesics in the handlebody $\mathfrak{X}_{\Gamma}$.

The homotopy quotient is given by

$$
\mathcal{S} \times_{\mathbb{Z}} \mathbb{R}=\mathcal{S}_{T}
$$

The $K$-theory of the $C^{*}$-algebra $(4.7)$ can be computed via the Pimsner-Voiculescu six terms exact sequence,

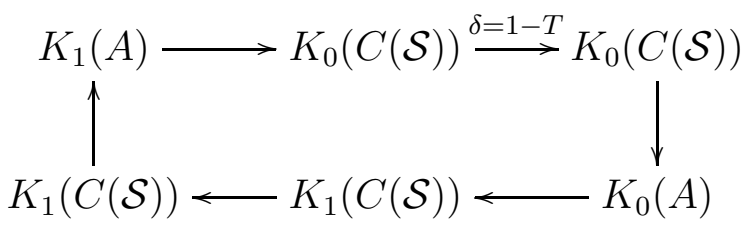

where $A=C(\mathcal{S}) \rtimes_{T} \mathbb{Z}$. Here, since the space $\mathcal{S}$ is totally disconnected, we have $K_{1}(C(\mathcal{S}))=0$ and $K_{0}(C(\mathcal{S}))=C(\mathcal{S}, \mathbb{Z})$ (locally constant integer valued functions). Thus the exact sequence becomes

$$
0 \rightarrow K_{1}(A) \rightarrow C(\mathcal{S}, \mathbb{Z}) \stackrel{\delta=1-T}{\longrightarrow} C(\mathcal{S}, \mathbb{Z}) \rightarrow K_{0}(A) \rightarrow 0
$$

and we obtain $K_{1}(A)=\mathbb{Z}=\operatorname{Ker}(\delta)$ and $K_{0}(A)=\operatorname{Coker}(\delta)$. In dynamical systems language, these are respectively the invariants and coinvariants of the invertible shift $T$ (cf. $[\mathbf{1 1}][\mathbf{9 5}])$.

In terms of the homotopy quotient, one can describe this exact sequence more geometrically in terms of the Thom isomorphism and the $\mu$-map

$$
\mu: K^{*+1}\left(\mathcal{S}_{T}\right) \cong H^{*+1}\left(\mathcal{S}_{T}, \mathbb{Z}\right) \rightarrow K_{*}\left(C(\mathcal{S}) \rtimes_{T} \mathbb{Z}\right) .
$$

Thus, we obtain:

$$
\begin{aligned}
& K_{1}(A) \simeq H^{0}\left(\mathcal{S}_{T}\right)=\mathbb{Z} \\
& K_{0}(A) \simeq H^{1}\left(\mathcal{S}_{T}\right)
\end{aligned}
$$

The $H^{1}\left(\mathcal{S}_{T}\right)$ can be identified with the Čech cohomology group given by the homotopy classes $\left[\mathcal{S}_{T}, U(1)\right]$, by mapping $f \mapsto[\exp (2 \pi i t f(x))]$, for $f \in C(\mathcal{S}, \mathbb{Z}) / \delta(C(\mathcal{S}, \mathbb{Z}))$. 
2.2. Filtration. The identification

$$
H^{1}\left(\mathcal{S}_{T}, \mathbb{Z}\right) \cong K_{0}\left(C(\mathcal{S}) \rtimes_{T} \mathbb{Z}\right)
$$

of the cohomology of $\mathcal{S}_{T}$ with the $K_{0}$-group of the crossed product $C^{*}$ algebra for the action of $T$ on $\mathcal{S}$ endows $H^{1}\left(\mathcal{S}_{T}, \mathbb{Z}\right)$ with a filtration.

THEOREM 2.1. The first cohomology of $\mathcal{S}_{T}$ is a direct limit

$$
H^{1}\left(\mathcal{S}_{T}\right)=\underset{n}{\lim } F_{n}
$$

of free abelian groups $F_{0} \hookrightarrow F_{1} \hookrightarrow \cdots F_{n} \hookrightarrow \cdots$ of ranks rank $F_{0}=2 g$ and rank $F_{n}=2 g(2 g-1)^{n-1}(2 g-2)+1$, for $n \geq 1$.

In fact, by the Pimsner-Voiculescu six terms exact sequence, the group $K_{0}\left(\mathrm{C}(\mathcal{S}) \rtimes_{T} \mathbb{Z}\right)$ can be identified with the cokernel of the map $1-T$ acting as $f \mapsto f-f \circ T$ on the $\mathbb{Z}$ module $\mathrm{C}(\mathcal{S}, \mathbb{Z}) \simeq K_{0}(C(\mathcal{S}))$.

Then the filtration is given by the submodules of functions depending only on the $a_{0} \ldots a_{n}$ coordinates in the doubly infinite words describing points in $\mathcal{S}$. Namely, one first shows that

$$
H^{1}\left(\mathcal{S}_{T}, \mathbb{Z}\right)=C(\mathcal{S}, \mathbb{Z}) / \delta(C(\mathcal{S}, \mathbb{Z}))=\mathcal{P} / \delta \mathcal{P}
$$

where $\mathcal{P}$ denotes the $\mathbb{Z}$-module of locally constant $\mathbb{Z}$-valued functions that depend only on "future coordinates". This can be identified with functions on the limit set $\Lambda_{\Gamma}$, since each point in $\Lambda_{\Gamma}$ is described by an infinite (to the right) admissible sequence in the generators $\gamma_{i}$ and their inverses. We have $\mathcal{P} \simeq C\left(\Lambda_{\Gamma}, \mathbb{Z}\right)$.

The module $\mathcal{P}$ clearly has a filtration by the submodules $\mathcal{P}_{n}$ of functions of the first $n+1$ coordinates. These have

$$
\operatorname{rank} \mathcal{P}_{n}=2 g(2 g-1)^{n} \text {. }
$$

We set $F_{n}:=\mathcal{P}_{n} / \delta \mathcal{P}_{n-1}$. One can show that there are induced injections $F_{n} \hookrightarrow F_{n+1}$ and that

$$
H^{1}\left(\mathcal{S}_{T}\right)=\underset{n}{\lim } F_{n}
$$

Moreover, we have $\operatorname{rank} F_{n}=\theta_{n}-\theta_{n-1}+1$, where $\theta_{n}$ is the number of admissible words of length $n+1$. All the $\mathbb{Z}$ modules $F_{n}$ and the quotients $F_{n} / F_{n-1}$ are torsion free $(c f .[\mathbf{9 5}])$.

2.3. Hilbert space and grading. It is convenient to consider the complex vector space

$$
\mathcal{P}_{\mathbb{C}}=C\left(\Lambda_{\Gamma}, \mathbb{Z}\right) \otimes \mathbb{C}
$$


and the corresponding exact sequence computing the cohomology with $\mathbb{C}$ coefficients:

$$
0 \rightarrow \mathbb{C} \rightarrow \mathcal{P}_{\mathbb{C}} \stackrel{\delta}{\longrightarrow} \mathcal{P}_{\mathbb{C}} \rightarrow H^{1}\left(\mathcal{S}_{T}, \mathbb{C}\right) \rightarrow 0
$$

The complex vector space $\mathcal{P}_{\mathbb{C}}$ sits in the Hilbert space

$$
\mathcal{P}_{\mathbb{C}} \subset \mathcal{L}=L^{2}\left(\Lambda_{\Gamma}, d \mu\right),
$$

where $\mu$ is the Patterson-Sullivan measure on the limit set, satisfying

$$
\gamma^{*} d \mu=\left|\gamma^{\prime}\right|^{\operatorname{dim}_{H}\left(\Lambda_{\Gamma}\right)} d \mu
$$

with $\operatorname{dim}_{H}\left(\Lambda_{\Gamma}\right)$ the Hausdorff dimension.

This gives a Hilbert space $\mathcal{L}$, together with a filtration $\mathcal{P}_{n}$ by finite dimensional subspaces. In such a setting, it is natural to consider a corresponding grading operator,

$$
D=\sum_{n} n \hat{\Pi}_{n}
$$

where $\Pi_{n}$ denotes the orthogonal projection onto $\mathcal{P}_{n}$ and $\hat{\Pi}_{n}=\Pi_{n}-$ $\Pi_{n-1}$.

2.4. Cuntz-Krieger algebra. There is a noncommutative space that encodes nicely the dynamics of the Schottky group $\Gamma$ on its limit set $\Lambda_{\Gamma}$. Consider the $2 g \times 2 g$ matrix $A$ that gives the admissibility condition for sequences in $\mathcal{S}$ : this is the matrix with $\{0,1\}$ entries satisfying $A_{i j}=1$ for $|i-j| \neq g$ and $A_{i j}=0$ otherwise.

The Cuntz-Krieger algebra $\mathcal{O}_{A}$ associated to this matrix is the universal $C^{*}$-algebra generated by partial isometries $S_{i}, i=1, \ldots, 2 g$, satisfying the relations

$$
\sum_{j} S_{j} S_{j}^{*}=1 \quad S_{i}^{*} S_{i}=\sum_{j} A_{i j} S_{j} S_{j}^{*} .
$$

Recall that a partial isometry is an operator $S$ satisfying $S=S S^{*} S$.

This algebra is related to the Schottky group by the following result.

Proposition 2.2. There is an isomorphism

$$
\mathcal{O}_{A} \cong C\left(\Lambda_{\Gamma}\right) \rtimes \Gamma \text {. }
$$

Up to a stabilization (tensoring with compact operators), the algebra has another crossed product description as

$$
\mathcal{O}_{A} \simeq \mathcal{F}_{A} \rtimes_{T} \mathbb{Z}
$$

with $\mathcal{F}_{A}$ an AF-algebra (a direct limit of finite dimensional $C^{*}$-algebras).

Consider the cochain complex of Hilbert spaces

$$
0 \rightarrow \mathbb{C} \rightarrow \mathcal{L} \stackrel{\delta}{\rightarrow} \mathcal{L} \rightarrow \mathcal{H} \rightarrow 0
$$


determined by the Pimsner-Voiculescu sequence (4.8).

Proposition 2.3. The $C^{*}$-algebra $\mathcal{O}_{A}$ admits a faithful representation on the Hilbert space $\mathcal{L}=L^{2}\left(\Lambda_{\Gamma}, d \mu\right)$.

This is obtained as follows.

For $d_{H}=\operatorname{dim}_{H}\left(\Lambda_{\Gamma}\right)$ the Hausdorff dimension, consider the operators

$$
P_{i} f=\chi_{\gamma_{i}} f \quad T_{i} f=\left|\left(\gamma_{i}^{-1}\right)^{\prime}\right|^{d_{H} / 2} f \circ \gamma_{i}^{-1},
$$

where $\left\{\gamma_{i}\right\}$ are the generators of $\Gamma$ and their inverses and

$$
T_{\gamma} f=\left|\gamma^{\prime}\right|^{d_{H} / 2} f \circ \gamma
$$

Then the operators

$$
S_{i}=\sum_{j} A_{i j} T_{i}^{*} P_{j}
$$

are partial isometries on $\mathcal{L}$ satisfying the Cuntz-Krieger relations for the matrix $A$ of the subshift of finite type (4.4). This gives the representation of $\mathcal{O}_{A}$.

2.5. Spectral triple for Schottky groups. On the Hilbert space $\mathcal{H}=\mathcal{L} \oplus \mathcal{L}$ consider the diagonal action of the algebra $\mathcal{O}_{A}$ and the Dirac operator $\mathcal{D}$ defined as

$$
\begin{aligned}
& \left.\mathcal{D}\right|_{\mathcal{L} \oplus 0}=\sum_{n}(n+1)\left(\hat{\Pi}_{n} \oplus 0\right) \\
& \left.\mathcal{D}\right|_{0 \oplus \mathcal{L}}=-\sum_{n} n\left(0 \oplus \hat{\Pi}_{n}\right) .
\end{aligned}
$$

The choice of the sign in (4.16) is not optimal from the point of view of the $K$-homology class. A better choice would be

$$
F=\left(\begin{array}{ll}
0 & 1 \\
1 & 0
\end{array}\right) \text {. }
$$

This would require in turn a modification of $|D|$. A construction along these lines is being considered in joint work of the author with Alina Vdovina and Gunther Cornelissen. In our setting here, the reason for the choice (4.16) in $[44]$ is the formula (4.91) relating $\mathcal{D}$ to the "logarithm of Frobenius" at arithmetic infinity.

TheOREm 2.4. For a Schottky group $\Gamma$ with $\operatorname{dim}_{H}\left(\Lambda_{\Gamma}\right)<1$, the data $\left(\mathcal{O}_{A}, \mathcal{H}, \mathcal{D}\right)$, for $\mathcal{H}=\mathcal{L} \oplus \mathcal{L}$ with the diagonal action of $\mathcal{O}_{A}$ through the representation (4.15) and the Dirac operator (4.16), define a nonfinitely summable, $\theta$-summable spectral triple. 
The key point of this result is the compatibility relation between the algebra and the Dirac operator, namely the fact that the commutators $[\mathcal{D}, a]$ are bounded operators, for all $a \in \mathcal{O}_{A}^{\text {alg }}$, the involutive subalgebra generated algebraically by the $S_{i}$ subject to the Cuntz-Krieger relations.

This follows by an estimate on the norm of the commutators $\left\|\left[\mathcal{D}, S_{i}\right]\right\|$ and $\left\|\left[\mathcal{D}, S_{i}^{*}\right]\right\|$, in terms of the Poincaré series of the Schottky group $\left(d_{H}<1\right)$

$$
\sum_{\gamma \in \Gamma}\left|\gamma^{\prime}\right|^{s}, \quad s=1>d_{H}
$$

where the Hausdorff dimension $d_{H}$ is the exponent of convergence of the Poincaré series.

The dimension of the $n$-th eigenspace of $\mathcal{D}$ is $2 g(2 g-1)^{n-1}(2 g-2)$ for $n \geq 1,2 g$ for $n=0$ and $2 g(2 g-1)^{-n-1}(2 g-2)$ for $n \leq-1$, so the spectral triple is not finitely summable, since $|\mathcal{D}|^{z}$ is not of trace class. It is $\theta$ summable, since the operator $\exp \left(-t \mathcal{D}^{2}\right)$ is of trace class, for all $t>0$.

Using the description (4.12) of the noncommutative space as crossed product of an AF algebra by the action of the shift, $\mathcal{F}_{A} \rtimes_{T} \mathbb{Z}$, one may be able to find a 1-summable spectral triple. Here the dense subalgebra should not contain any of the group elements. In fact, by the result of [20], the group $\Gamma$ is a free group, hence its growth is too fast to support finitely summable spectral triples on its group ring.

\section{Arithmetic infinity: archimedean primes}

An algebraic number field $\mathbb{K}$, which is an extension of $\mathbb{Q}$ such that $[\mathbb{K}: \mathbb{Q}]=n$, admits $n=r_{1}+2 r_{2}$ embeddings

$$
\alpha: \mathbb{K} \hookrightarrow \mathbb{C} .
$$

These can be subdivided into $r_{1}$ real embeddings $\mathbb{K} \hookrightarrow \mathbb{R}$ and $r_{2}$ pairs of complex conjugate embeddings. The embeddings (4.17) are called the archimedean primes of the number field. The set of archimedean primes is often referred to as "arithmetic infinity", a terminology borrowed from the case of the unique embedding of $\mathbb{Q} \hookrightarrow \mathbb{R}$, which is called the "infinite prime".

A general strategy in arithmetic geometry is to adapt the tools of classical algebraic geometry to the arithmetic setting. In particular, over $\mathbb{Q}$ the set of primes $\operatorname{Spec}(\mathbb{Z})$ is the analog in arithmetic geometry of the affine line. It becomes clear then that some compactification is necessary, at least in order to have a well behaved form of intersection theory in arithmetic geometry. Namely, we need to pass from the 
"affine" $\operatorname{Spec}(\mathbb{Z})$ to the projective case. The compactification is obtained by adding the infinite prime to the set of finite primes. A goal of arithmetic geometry then becomes developing a setting that treats the infinite prime and the finite primes of equal footing.

More generally, for a number field $\mathbb{K}$ with $O_{\mathbb{K}}$ its ring of integers, the set of primes $\operatorname{Spec}\left(O_{\mathbb{K}}\right)$ is compactified by adding the set of "archimedean primes"

$$
\overline{\operatorname{Spec}\left(O_{\mathbb{K}}\right)}=\operatorname{Spec}\left(O_{\mathbb{K}}\right) \cup\{\alpha: \mathbb{K} \hookrightarrow \mathbb{C}\} .
$$

3.1. Arithmetic surfaces. Let $X$ be a smooth projective algebraic curve defined over $\mathbb{Q}$. Then, by clearing denominators one obtains an equation with $\mathbb{Z}$ coefficients. This determines a scheme $X_{\mathbb{Z}}$ over $\operatorname{Spec}(\mathbb{Z})$,

$$
X_{\mathbb{Z}} \otimes_{\mathbb{Z}} \operatorname{Spec}(\mathbb{Q})=X,
$$

where the closed fiber of $X_{\mathbb{Z}}$ at a prime $p \in \operatorname{Spec}(\mathbb{Z})$ is the reduction of $X \bmod p$. Thus, viewed as an arithmetic variety, an algebraic curve becomes a 2-dimensional fibration over the affine line $\operatorname{Spec}(\mathbb{Z})$.

One can also consider reductions of $X$ (defined over $\mathbb{Z}$ ) modulo $p^{n}$ for some prime $p \in \operatorname{Spec}(\mathbb{Z})$. The limit as $n \rightarrow \infty$ defines a $p$ adic completion of $X_{\mathbb{Z}}$. This can be thought of as an "infinitesimal neighborhood" of the fiber at $p$.

The picture is more complicated at arithmetic infinity, since one does not have a suitable notion of "reduction mod $\infty$ " available to define the closed fiber. On the other hand, one does have the analog of the $p$-adic completion at hand. This is given by the Riemann surface (smooth projective algebraic curve over $\mathbb{C}$ ) determined by the equation of the algebraic curve $X$ over $\mathbb{Q}$, under the embedding of $\mathbb{Q} \subset \mathbb{C}$,

$$
X(\mathbb{C})=X \otimes_{\mathbb{Q}} \operatorname{Spec}(\mathbb{C})
$$

with the absolute value $|\cdot|$ at the infinite prime replacing the $p$-adic valuations.

Similarly, for $\mathbb{K}$ a number field and $O_{\mathbb{K}}$ its ring of integers, a smooth proper algebraic curve $X$ over $\mathbb{K}$ determines a smooth minimal model $X_{O_{\mathbb{K}}}$, which defines an arithmetic surface $\mathcal{X}_{O_{\mathbb{K}}}$ over $\operatorname{Spec}\left(O_{\mathbb{K}}\right)$. The closed fiber $X_{\wp}$ of $\mathcal{X}_{O_{\mathbb{K}}}$ over a prime $\wp \in O_{\mathbb{K}}$ is given by the reduction $\bmod \wp$.

When $\operatorname{Spec}\left(O_{\mathbb{K}}\right)$ is compactified by adding the archimedean primes, one also obtains $n$ Riemann surfaces $X_{\alpha}(\mathbb{C})$, obtained from the equation defining $X$ over $\mathbb{K}$ under the embeddings $\alpha: \mathbb{K} \hookrightarrow \mathbb{C}$. Of these Riemann surfaces, $r_{1}$ carry a real involution. 


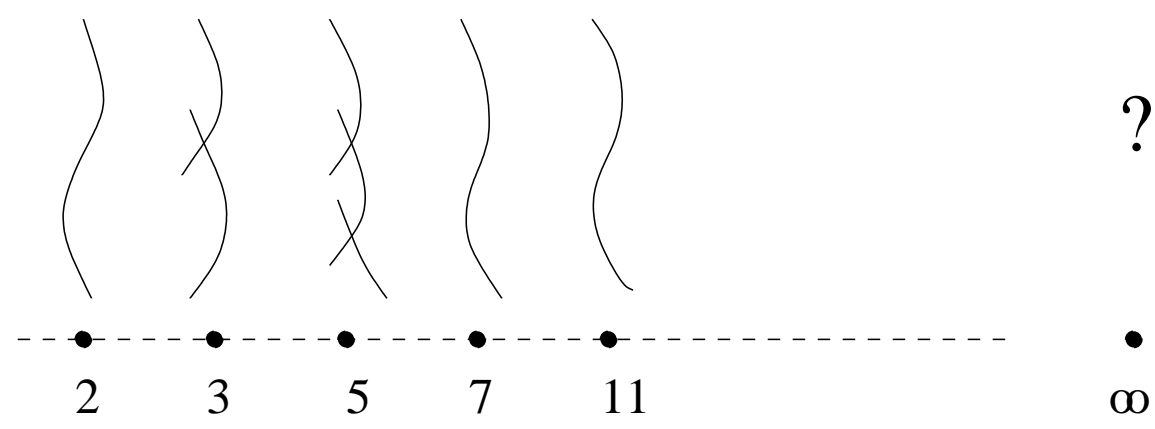

FiguRE 7. Arithmetic surface over $\operatorname{Spec}(\mathbb{Z})$

Thus, the picture of an arithmetic surface over $\overline{\operatorname{Spec}\left(O_{\mathbb{K}}\right)}$ is as follows:

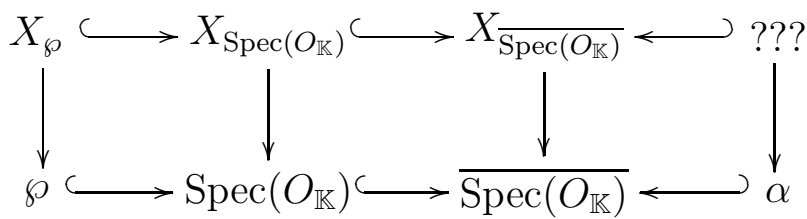

where we do not have an explicit geometric description of the closed fibers over the archimedean primes (Figure 7).

Formally, one can enlarge the group of divisors on the arithmetic surface by adding formal real linear combinations of irreducible "closed vertical fibers at infinity" $\sum_{\alpha} \lambda_{\alpha} F_{\alpha}$. Here the fibers $F_{\alpha}$ are only treated as formal symbols, and no geometric model of such fibers is provided. The remarkable fact is that Hermitian geometry on the Riemann surfaces $X_{\alpha}(\mathbb{C})$ is sufficient to specify the contribution of such divisors to intersection theory on the arithmetic surface, even without an explicit knowledge of the closed fiber.

The main idea of Arakelov geometry is that it is sufficient to work with the "infinitesimal neighborhood" $X_{\alpha}(\mathbb{C})$ of the fibers $F_{\alpha}$, to have well defined intersection indices.

If one thinks, by analogy, of the case of the classical geometry of a degeneration of algebraic curves over a disk $\Delta$ with a special fiber over 0 , the analogous statement would be saying that the geometry of the special fiber is completely determined by the generic fiber. This is a very strong statement on the form of the degeneration: for instance blowing up points on the special fiber is not seen by just looking at the generic fiber. Investigating this analogy leads one to expect that the fiber at infinity should behave like the totally degenerate case. This is the case where one has maximal degeneration, where all the components of the closed fiber are $\mathbb{P}^{1}$ 's and the geometry of the degeneration 
is completely encoded by the dual graph, which describes in a purely combinatorial way how these $\mathbb{P}^{1}$ 's are joined. The dual graph has a vertex for each component of the closed fiber and an edge for each double point.

The local intersection multiplicities of two finite, horizontal, irreducible divisors $D_{1}, D_{2}$ on $X_{O_{\mathbb{K}}}$ is given by

$$
\left[D_{1}, D_{2}\right]=\left[D_{1}, D_{2}\right]_{f i n}+\left[D_{1}, D_{2}\right]_{\text {inf }}
$$

where the first term counts the contribution from the finite places (i.e. what happens over $\operatorname{Spec}\left(O_{\mathbb{K}}\right)$ ) and the second term is the contribution of the archimedean primes, i.e. the part of the intersection that happens over arithmetic infinity.

While the first term is computed in algebro-geometric terms, from the local equations for the divisors $D_{i}$ at $P$, the second term is defined as a sum of values of Green functions $g_{\alpha}$ on the Riemann surfaces $X_{\alpha}(\mathbb{C})$,

$$
\left[D_{1}, D_{2}\right]_{i n f}=-\sum_{\alpha} \epsilon_{\alpha}\left(\sum_{\beta, \gamma} g_{\alpha}\left(P_{1, \beta}^{\alpha}, P_{2, \gamma}^{\alpha}\right)\right)
$$

at points

$$
\left\{P_{i, \beta}^{\alpha} \mid \beta=1, \ldots,\left[\mathbb{K}\left(D_{i}\right): \mathbb{K}\right]\right\} \subset X_{\alpha}(\mathbb{C})
$$

for a finite extension $\mathbb{K}\left(D_{i}\right)$ of $\mathbb{K}$ determined by $D_{i}$. Here $\epsilon_{\alpha}=1$ for real embeddings and $=2$ for complex embeddings.

For a detailed account of these notions of Arakelov geometry, one can refer to $[\mathbf{5 0}][\mathbf{7 1}]$.

Further evidence for the similarity between the archimedean and the totally degenerate fibers came from an explicit computation of the Green function at the archimedean places derived by Manin [79] in terms of a Schottky uniformization of the Riemann surface $X_{\alpha}(\mathbb{C})$. Such a uniformization has an analog at a finite prime, in terms of p-adic Schottky groups, only in the totally degenerate case. Another source of evidence comes from a cohomological theory of the local factors at the archimedean primes, developed by Consani [43], which shows that the resulting description of the local factor as regularized determinant at the archimedean primes resembles closely the case of the totally degenerate reduction at a finite prime.

We will present both results in the light of the noncommutative space $\left(\mathcal{O}_{A}, \mathcal{H}, \mathcal{D}\right)$ introduced in the previous section. As shown by Consani and the author in $[44][45][46][47]$ the noncommutative geometry of this space is naturally related to both Manin's result on the Arakelov Green function and the cohomological construction of Consani. 


\section{Arakelov geometry and hyperbolic geometry}

In this section we give a detailed account of Manin's result [79] on the relation between the Arakelov Green function on a Riemann surface $X(\mathbb{C})$ with Schottky uniformization and geodesics in the 3-dimensional hyperbolic handlebody $\mathfrak{X}_{\Gamma}$. Our exposition follows closely the seminal paper [79].

4.1. Arakelov Green function. Given a divisor $A=\sum_{x} m_{x}(x)$ with support $|A|$ on a smooth compact Riemann surface $X(\mathbb{C})$, and a choice of a positive real-analytic 2-form $d \mu$ on $X(\mathbb{C})$, the Green function $g_{\mu, A}=g_{A}$ is a real analytic function on on $X(\mathbb{C}) \backslash|A|$, uniquely determined by the following conditions:

- Laplace equation: $g_{A}$ satisfies $\partial \bar{\partial} g_{A}=\pi i\left(\operatorname{deg}(A) d \mu-\delta_{A}\right)$, with $\delta_{A}$ the $\delta$-current $\varphi \mapsto \sum_{x} m_{x} \varphi(x)$.

- Singularities: if $z$ is a local coordinate in a neighborhood of $x$, then $g_{A}-m_{x} \log |z|$ is locally real analytic.

- Normalization: $g_{A}$ satisfies $\int_{X} g_{A} d \mu=0$.

If $B=\sum_{y} n_{y}(y)$ is another divisor, such that $|A| \cap|B|=\emptyset$, then the expression $g_{\mu}(A, B):=\sum_{y} n_{y} g_{\mu, A}(y)$ is symmetric and biadditive in $A, B$. Generally, such an expression $g_{\mu}$ depends on $\mu$, where the choice of $\mu$ is equivalent to the choice of a real analytic Riemannian metric on $X(\mathbb{C})$, compatible with the complex structure.

However, in the special case of degree zero divisors, $\operatorname{deg} A=\operatorname{deg} B=$ 0 , the $g_{\mu}(A, B)=g(A, B)$ are conformal invariants.

In the case on the Riemann sphere $\mathbb{P}^{1}(\mathbb{C})$, if $w_{A}$ is a meromorphic function with $\operatorname{Div}\left(w_{A}\right)=A$, we have

$$
g(A, B)=\log \prod_{y \in|B|}\left|w_{A}(y)\right|^{n_{y}}=\operatorname{Re} \int_{\gamma_{B}} \frac{d w_{A}}{w_{A}},
$$

where $\gamma_{B}$ is a 1 -chain with boundary $B$.

In the case of degree zero divisors $A, B$ on a Riemann surface of higher genus, the formula (4.19) can be generalized, replacing the logarithmic differential $d w_{A} / w_{A}$ with a differential of the third kind (meromorphic differential with nonvanishing residues) $\omega_{A}$ with purely imaginary periods and residues $m_{x}$ at $x$. This gives

$$
g(A, B)=\operatorname{Re} \int_{\gamma_{B}} \omega_{A} .
$$

Thus, one can explicitly compute $g(A, B)$ from a basis of differentials of the third kind with purely imaginary periods. 


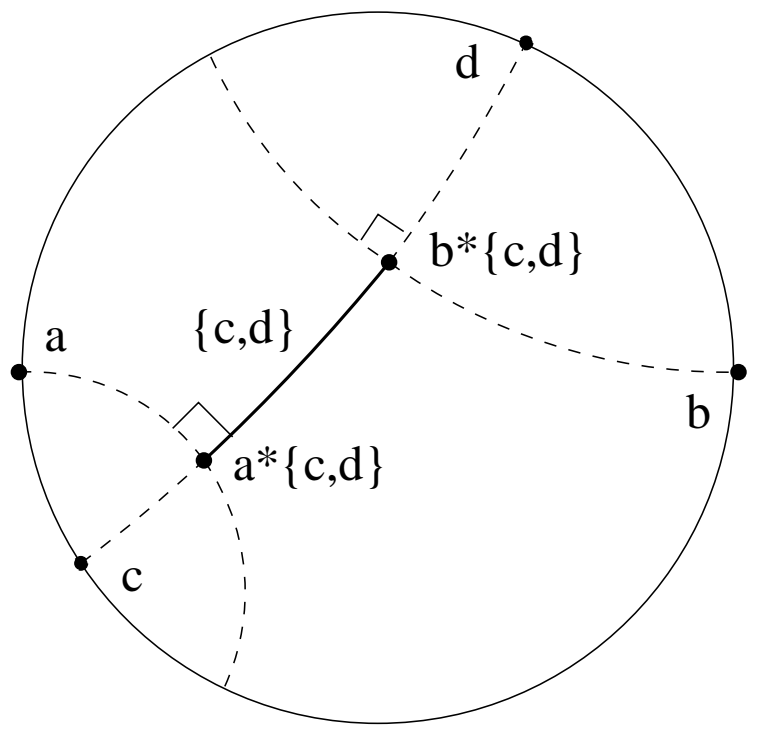

Figure 8. Cross ratio and geodesic length

4.2. Cross ratio and geodesics. The basic step leading to the result of Manin, expressing the Arakelov Green function in terms of geodesics in the hyperbolic handlebody $\mathfrak{X}_{\Gamma}$, is a very simple classical fact of hyperbolic geometry, namely the fact that the cross ratio of four points on $\mathbb{P}^{1}(\mathbb{C})$ can be expressed in terms of geodesics in the 'interior' $\mathbb{H}^{3}$ :

$$
\log |\langle a, b, c, d\rangle|=- \text { ordist }(a *\{c, d\}, b *\{c, d\}) .
$$

Here, following [79], ordist denotes the oriented distance, and we use the notation $a *\{c, d\}$ to indicate the point on the geodesic $\{c, d\}$ in $\mathbb{H}^{3}$ with endpoints $c, d \in \mathbb{P}^{1}(\mathbb{C})$, obtained as the intersection of $\{c, d\}$ with the unique geodesic from $a$ that cuts $\{c, d\}$ at a right angle (Figure 8).

4.3. Differentials and Schottky uniformization. The next important step in Manin's result [79] is to show that, if $X(\mathbb{C})=\Gamma \backslash \Omega_{\Gamma}$ is a Riemann surface with a Schottky uniformization, then one obtains a basis of differentials of the third kind with purely imaginary periods, by taking suitable averages over the group $\Gamma$ of expressions involving the cross ratio of points on $\mathbb{P}^{1}(\mathbb{C})$.

We denote by $C(\mid \gamma)$ a set of representatives of $\Gamma /\left(\gamma^{\mathbb{Z}}\right)$, by $C(\rho \mid \gamma)$ a set of representatives for $\left(\rho^{\mathbb{Z}}\right) \backslash \Gamma /\left(\gamma^{\mathbb{Z}}\right)$, and by $S(\gamma)$ the conjugacy class of $\gamma$ in $\Gamma$.

Let $w_{A}$ be a meromorphic function on $\mathbb{P}^{1}(\mathbb{C})$ with divisor $A=$ $(a)-(b)$, such that the support $|A|$ is contained in the complement of 
an open neighborhood of $\Lambda_{\Gamma}$. We use the notation

$$
\langle a, b, c, d\rangle:=\frac{(a-b)(c-d)}{(a-d)(c-b)}
$$

for the cross-ratio of points $a, b, c, d \in \mathbb{P}^{1}(\mathbb{C})$.

For a fixed choice of a base point $z_{0} \in \Omega_{\Gamma}$, the series

$$
\nu_{(a)-(b)}:=\sum_{\gamma \in \Gamma} d \log \left\langle a, b, \gamma z, \gamma z_{0}\right\rangle
$$

gives the lift to $\Omega_{\Gamma}$ of a differential of the third kind on the Riemann surface $X(\mathbb{C})$, endowed with the choice of Schottky uniformization. These differentials have residues \pm 1 at the images of $a$ and $b$ in $X(\mathbb{C})$, and they have vanishing $a_{k}$ periods, where $\left\{a_{k}, b_{k}\right\}_{k=1 \ldots g}$ are the generators of the homology of $X(\mathbb{C})$.

Similarly, we obtain lifts of differentials of the first kind on $X(\mathbb{C})$, by considering the series

$$
\omega_{\gamma}=\sum_{h \in C(\mid \gamma)} d \log \left\langle h z^{+}(\gamma), h z^{-}(\gamma), z, z_{0}\right\rangle
$$

where we denote by $\left\{z^{+}(\gamma), z^{-}(\gamma)\right\} \subset \Lambda_{\Gamma}$ the pair consisting of the attractive and repelling fixed points of $\gamma \in \Gamma$.

The series (4.23) and (4.24) converge absolutely on compact sets $K \subset \Omega_{\Gamma}$, whenever $\operatorname{dim}_{H} \Lambda_{\Gamma}<1$. Moreover, they do not depend on the choice of the base point $z_{0} \in \Omega_{\Gamma}$.

In particular, given a choice $\left\{\gamma_{k}\right\}_{k=1}^{g}$ of generators of the Schottky group $\Gamma$, we obtain by (4.24) a basis of holomorphic differentials $\omega_{\gamma_{k}}$, that satisfy

$$
\int_{a_{k}} \omega_{\gamma_{\ell}}=2 \pi \sqrt{-1} \delta_{k \ell}
$$

One can then use a linear combination of the holomorphic differentials $\omega_{\gamma_{k}}$ to correct the meromorphic differentials $\nu_{(a)-(b)}$ in such a way that the resulting meromorphic differentials have purely imaginary $b_{k}$-periods. Let $X_{\ell}(a, b)$ be coefficients such that the differentials of the third kind

$$
\omega_{(a)-(b)}:=\nu_{(a)-(b)}-\sum_{\ell} X_{\ell}(a, b) \omega_{g_{\ell}}
$$


have purely imaginary $b_{k}$-periods. The coefficients $X_{\ell}(a, b)$ satisfy the system of equations

$$
\sum_{\ell=1}^{g} X_{\ell}(a, b) \Re\left(\tau_{k l}\right)=\Re \int_{b_{k}} \nu_{(a)-(b)}=\sum_{h \in S\left(g_{k}\right)} \log \left|\left\langle a, b, z^{+}(h), z^{-}(h)\right\rangle\right| .
$$

Thus, one obtains $([\mathbf{7 9}], c f$. also [109]) from (4.20) and (4.26) that the Arakelov Green function for $X(\mathbb{C})$ with Schottky uniformization can be computed as

$$
\begin{gathered}
g((a)-(b),(c)-(d))=\sum_{h \in \Gamma} \log |\langle a, b, h c, h d\rangle| \\
-\sum_{\ell=1}^{g} X_{\ell}(a, b) \sum_{h \in S\left(g_{\ell}\right)} \log \left|\left\langle z^{+}(h), z^{-}(h), c, d\right\rangle\right| .
\end{gathered}
$$

Notice that this result seems to indicate that there is a choice of Schottky uniformization involved as additional data for Arakelov geometry at arithmetic infinity. However, as we remarked previously, at least in the case of archimedean primes that are real embeddings (as is the case of arithmetic infinity for $\mathbb{Q}$ ), the Schottky uniformization is determined by the real structure, by splitting $X(\mathbb{C})$ along the real locus $X(\mathbb{R})$, when the latter is nonempty.

4.4. Green function and geodesics. By combining the basic formula (4.21) with the formula (4.28) for the Green function on a Riemann surface with Schottky uniformization one can replace each term appearing in (4.28) with a corresponding term which computes the oriented geodesic length of a certain arc of geodesic in $\mathfrak{X}_{\Gamma}$ :

$$
\begin{gathered}
g((a)-(b),(c)-(d))=-\sum_{h \in \Gamma} \operatorname{ordist}(a *\{h c, h d\}, b *\{h c, h d\}) \\
+\sum_{\ell=1}^{g} X_{\ell}(a, b) \sum_{h \in S\left(g_{\ell}\right)} \operatorname{ordist}\left(z^{+}(h) *\{c, d\}, z^{-}(h) *\{c, d\}\right) .
\end{gathered}
$$

The coefficients $X_{\ell}(a, b)$ can also be expressed in terms of geodesics, using the equation (4.27).

\section{Intermezzo: Quantum gravity and black holes}

Anti-de Sitter space $\mathrm{AdS}_{d+1}$ is a highly symmetric space-time, satisfying Einstein's equations with constant curvature $R<0$. Physically it describes empty space with a negative cosmological constant. In order to avoid time-like closed geodesics, it is customary to pass to the universal cover $\widetilde{\operatorname{AdS}}_{d+1}$, whose boundary at infinity of the is a compactification of $d$-dimensional Minkowski space-time. When passing to Euclidean signature, $\widetilde{\operatorname{AdS}}_{d+1}$ becomes the real hyperbolic space $\mathbb{H}^{d+1}$. 
The $(3+1)$-dimensional Anti-de Sitter space is a well known example of space-time in general relativity. Topologically $\mathrm{AdS}_{3+1}$ is of the form $S^{1} \times \mathbb{R}^{3}$, while metrically it is realized by the hyperboloid $-u^{2}-v^{2}+x^{2}+y^{2}+z^{2}=1$ in $\mathbb{R}^{5}$, with the metric element $d s^{2}=-d u^{2}-d v^{2}+d x^{2}+d y^{2}+d z^{2}$. The universal cover is topologically $\mathbb{R}^{4}$. In the context of quantum gravity, it is especially interesting to consider the case of the $(2+1)$-dimensional Anti-de Sitter space $\mathrm{AdS}_{2+1}$ and its Euclidean counterpart, the real 3-dimensional hyperbolic space $\mathbb{H}^{3}$.

The holographic principle postulates the existence of an explicit correspondence between gravity on a bulk space which is asymptotically $\widetilde{\operatorname{AdS}}_{d+1}$ (e.g. a space obtained as a global quotient of $\widetilde{\operatorname{AdS}}_{d+1}$ by a discrete group of isometries) and field theory on its conformal boundary at infinity.

The relation (4.21), which identifies the Green function

$$
g((a)-(b),(c)-(d))
$$

on $\mathbb{P}^{1}(\mathbb{C})$ with the oriented length of a geodesic arc in $\mathbb{H}^{3}$, can be thought of as an instance of this holography principle, when we interpret one side as geodesic propagator on the bulk space in a semiclassical approximation, and the other side (the Green function) as the two-point correlation function of the boundary field theory. Notice that, because of the prescribed behavior of the Green function at the singularities given by the points of the divisor, our "four points" invariant $g((a)-(b),(c)-(d))$, when $a \rightarrow c$ and $b \rightarrow d$, gives the two-point correlator with a logarithmic divergence which is intrinsic and does not depend on a choice of cut-off function (unlike the way regularization is often used in the physics literature).

In [82] we showed that the relation (4.29) between Arakelov Green functions and configurations of geodesics in the hyperbolic handlebody, proved by Manin in [79], provides in fact precisely the correspondence prescribed by the holography principle, for a class of $2+1$ dimensional space-times known as Euclidean Krasnov black holes. These include the Bañados-Teitelboim-Zanelli black holes: an important class of space-times in the context of $(2+1)$-dimensional quantum gravity.

5.1. Bañados-Teitelboim-Zanelli black hole. We consider the case of a hyperbolic handlebody of genus one (a solid torus), with conformal boundary at infinity given by an elliptic curve.

Recall that we can describe elliptic curves via the Jacobi uniformization. (We have already encountered it in the previous lectures, in the context of non-commutative elliptic curves.) Let $X_{q}(\mathbb{C})=\mathbb{C}^{*} / q^{\mathbb{Z}}$ be 

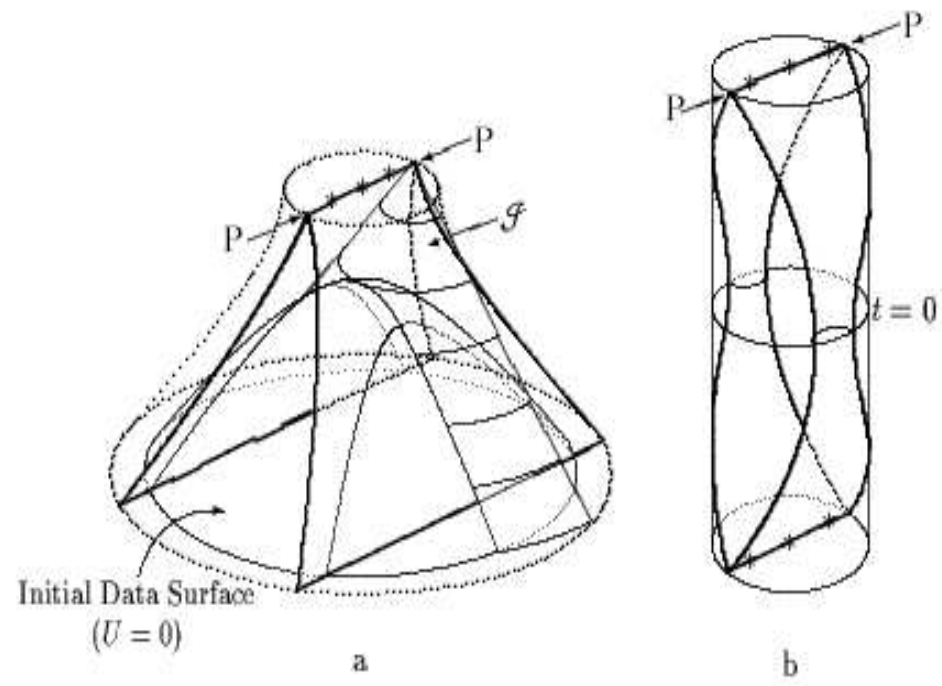

FiguRE 9. Minkowskian BTZ black hole

such a description of an elliptic curve, where $q$ is a hyperbolic element of $\operatorname{PSL}(2, \mathbb{C})$ with fixed points $\{0, \infty\}$ on the sphere at infinity $\mathbb{P}^{1}(\mathbb{C})$ of $\mathbb{H}^{3}$, that is, $q \in \mathbb{C}^{*}$ with $|q|<1$. The action of $q$ on $\mathbb{P}^{1}(\mathbb{C})$ extends to an action on $\overline{\mathbb{H}}^{3}=\mathbb{H}^{3} \cup \mathbb{P}^{1}(\mathbb{C})$ by

$$
q:(z, y) \mapsto(q z,|q| y) .
$$

It is easy to see that the quotient by this action

$$
\mathfrak{X}_{q}=\mathbb{H}^{3} / q^{\mathbb{Z}}
$$

is topologically a solid torus, compactified at infinity by the conformal boundary $X_{q}(\mathbb{C})$.

The space $\mathfrak{X}_{q}$ is well known in the physics literature as the Euclidean Bañados-Teitelboim-Zanelli black hole, where the parameter $q \in \mathbb{C}^{*}$ is written in the form

$$
q=\exp \left(\frac{2 \pi\left(i\left|r_{-}\right|-r_{+}\right)}{\ell}\right)
$$

with

$$
r_{ \pm}^{2}=\frac{1}{2}\left(M \ell \pm \sqrt{M^{2} \ell^{2}+J}\right) .
$$

Here $M$ and $J$ are the mass and angular momentum of the rotating black hole, and $-1 / \ell^{2}$ is the cosmological constant. The corresponding black hole in Minkowskian signature is illustrated in Figure $9^{2}$. 

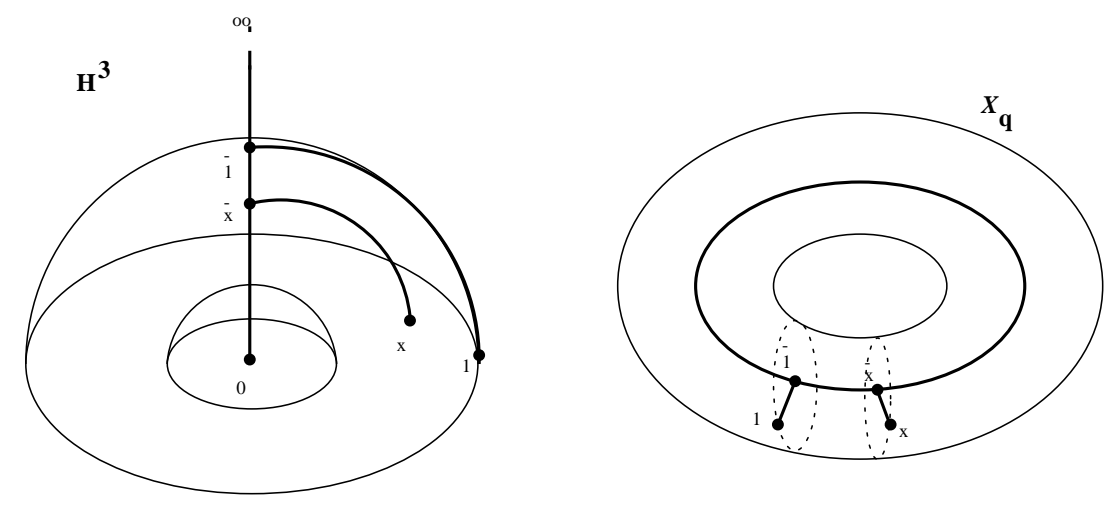

Figure 10. Geodesics in Euclidean BTZ black holes

In the case of the elliptic curve $X_{q}(\mathbb{C})=\mathbb{C}^{*} / q^{\mathbb{Z}}$, a formula of Alvarez-Gaumé, Moore, and Vafa [2] gives the operator product expansion of the path integral for bosonic field theory as

$$
g(z, 1)=\log \left(|q|^{B_{2}(\log |z| / \log |q|) / 2}|1-z| \prod_{n=1}^{\infty}\left|1-q^{n} z\right|\left|1-q^{n} z^{-1}\right|\right) .
$$

This is in fact the Arakelov Green function on $X_{q}(\mathbb{C})$. In terms of geodesics in the Euclidean BTZ black hole this becomes $(c f .[\mathbf{7 9}])$

$$
g(z, 1)=-\frac{1}{2} \ell\left(\gamma_{0}\right) B_{2}\left(\frac{\ell_{\gamma_{0}}(\bar{z}, \overline{1})}{\ell\left(\gamma_{0}\right)}\right)+\sum_{n \geq 0} \ell_{\gamma_{1}}\left(\overline{0}, \bar{z}_{n}\right)+\sum_{n \geq 1} \ell_{\gamma_{1}}\left(\overline{0}, \tilde{z}_{n}\right) .
$$

Here $B_{2}(v)=v^{2}-v+1 / 6$ is the second Bernoulli polynomial. In the formula we are using the notation $\bar{x}=x *\{0, \infty\} ; \bar{z}_{n}=q^{n} z *\{1, \infty\}$, $\tilde{z}_{n}=q^{n} z^{-1} *\{1, \infty\}$ as in [79], as illustrated in Figure 10 and 11 . These terms describe gravitational properties of the Euclidean BTZ black hole. For instance, $\ell\left(\gamma_{0}\right)$ measures the black hole entropy. The whole expression is a combination of geodesic propagators.

5.2. Krasnov black holes. The problem of computing the bosonic field propagator on an algebraic curve $X_{\mathbb{C}}$ can be solved by providing differentials of the third kind with purely imaginary periods

$$
\omega_{(a)-(b)}:=\nu_{(a)-(b)}-\sum_{l} X_{l}(a, b) \omega_{g_{l}},
$$

\footnotetext{
${ }^{2}$ Figure 9 is taken from $[\mathbf{3}]$.
} 

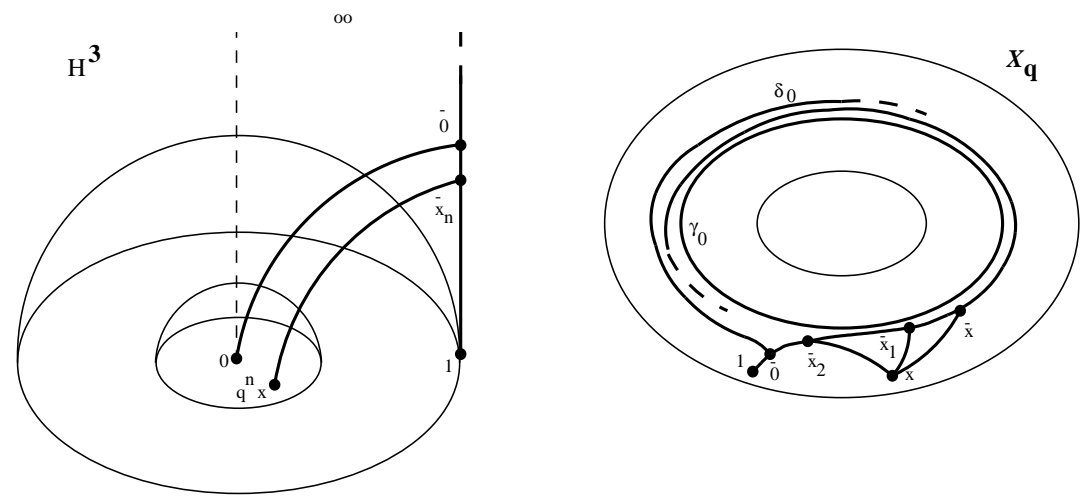

Figure 11. Geodesics in Euclidean BTZ black holes

hence it can be related directly to the problem of computing the Arakelov Green function.

Differentials as above then determine all the higher correlation functions

$$
G\left(z_{1}, \ldots, z_{m} ; w_{1}, \ldots, w_{\ell}\right)=\sum_{j=1}^{m} \sum_{i=1}^{\ell} q_{i}\left\langle\phi\left(z_{i}, \bar{z}_{i}\right) \phi\left(w_{j}, \bar{w}_{j}\right)\right\rangle q_{j}^{\prime}
$$

for $q_{i}$ a system of charges at positions $z_{i}$ interacting with charges $q_{j}^{\prime}$ at positions $w_{j}$, from the basic two-point correlator $G_{\mu}(a-b, z)$ given by the Green function expressed in terms of the differentials $\omega_{(a)-(b)}(z)$. When we use a Schottky uniformization, we obtain the differentials $\omega_{(a)-(b)}$ as in (4.26).

The bulk space corresponding to the conformal boundary $X(\mathbb{C})$ is given by the hyperbolic handlebody $\mathfrak{X}_{\Gamma}$. As in the case of the BTZ black hole, it is possible to interpret these real hyperbolic 3-manifolds as analytic continuations to Euclidean signature of Minkowskian black holes that are global quotients of $\mathrm{AdS}_{2+1}$. This is not just the effect of the usual rotation from Minkowskian to Euclidean signature, but a more refined form of "analytic continuation" which is adapted to the action of the Schottky group, and which was introduced by Kirill Krasnov [65] [66] in order to deal with this class of space-times.

The formula (4.29) then gives the explicit bulk/boundary correspondence of the holography principle for this class of space-times: each term in the Bosonic field propagator for $X_{\mathbb{C}}$ is expressed in terms of geodesics in the Euclidean Krasnov black hole $\mathfrak{X}_{\Gamma}=\mathbb{H}^{3} / \Gamma$. 


\section{Dual graph and noncommutative geometry}

Manin's result on the Arakelov Green function and hyperbolic geometry suggests a geometric model for the dual graph of the mysterious fiber at arithmetic infinity.

In fact, the result discussed above on the Green function has an analog, due to Drinfel'd and Manin [56] in the case of a finite prime with a totally split fiber. This is the case where the $p$-adic completion admits a Schottky uniformization by a $p$-adic Schottky group.

6.1. Schottky-Mumford curves. If $K$ is a given finite extension of $\mathbb{Q}_{p}$, we denote by $\mathcal{O} \subset K$ its ring of integers, by $\mathfrak{m} \subset \mathcal{O}$ the maximal ideal, and by $k$ the residue field $k=\mathcal{O} / \mathfrak{m}$. This is a finite field of cardinality $q=\operatorname{card}(\mathcal{O} / \mathfrak{m})$.

It is well known that a curve $X$ over a finite extension $K$ of $\mathbb{Q}_{p}$, which is $k$-split degenerate, for $k$ the residue field, admits a $p$-adic uniformization by a $p$-adic Schottky group $\Gamma$ acting on the Bruhat-Tits tree $\Delta_{K}$.

The Bruhat-Tits tree is obtained by considering free $\mathcal{O}$-modules of rank 2, with the equivalence relation that $M_{1} \sim M_{2}$ iff $\exists \lambda \in K^{*}$ such that $M_{1}=\lambda M_{2}$. The vertices of the tree consist of these equivalence classes. A distance is introduced on this set by prescribing that if $\left\{M_{1}\right\},\left\{M_{2}\right\} \in \Delta_{K}^{0}$, with $M_{1} \supset M_{2}$, then

$$
M_{1} / M_{2} \simeq \mathcal{O} / \mathfrak{m}^{l} \oplus \mathcal{O} / \mathfrak{m}^{k}, \quad l, k \in \mathbb{N}
$$

and we set the distance to be $d\left(\left\{M_{1}\right\},\left\{M_{2}\right\}\right)=|l-k|$. We form the tree $\Delta_{K}$, by adding an edge between any two pairs of vertices with $d\left(\left\{M_{1}\right\},\left\{M_{2}\right\}\right)=1$. This gives a connected, locally finite tree with $q+1$ edges departing from each vertex. The group $\operatorname{PGL}(2, K)$ acts transitively (on the left) by isometries.

The Bruhat-Tits tree $\Delta_{K}$ is the analog of the 3-dimensional real hyperbolic space $\mathbb{H}^{3}$ at the infinite primes. The set of ends of $\Delta_{K}$ is identified with $\mathbb{P}^{1}(K)$, just as we have $\mathbb{P}^{1}(\mathbb{C})=\partial \mathbb{H}^{3}$ in the case at infinity.

A $p$-adic Schottky group $\Gamma$ is a discrete subgroup of $\operatorname{PGL}(2, K)$ which consists of hyperbolic elements $\gamma$ (the eigenvalues of $\gamma$ in $K$ have different valuation), and which is isomorphic to a free group in $g$ generators. We still denote by $\Lambda_{\Gamma} \subset \mathbb{P}^{1}(K)$ the limit set, that is, the closure of the set of points in $\mathbb{P}^{1}(K)$ that are fixed points of some $\gamma \in \Gamma \backslash\{1\}$. As in the case at infinity, we have $\operatorname{card}\left(\Lambda_{\Gamma}\right)<\infty$ if and only if $\Gamma=(\gamma)^{\mathbb{Z}}$, for some $\gamma \in \Gamma$ (the genus one case). We denote by 


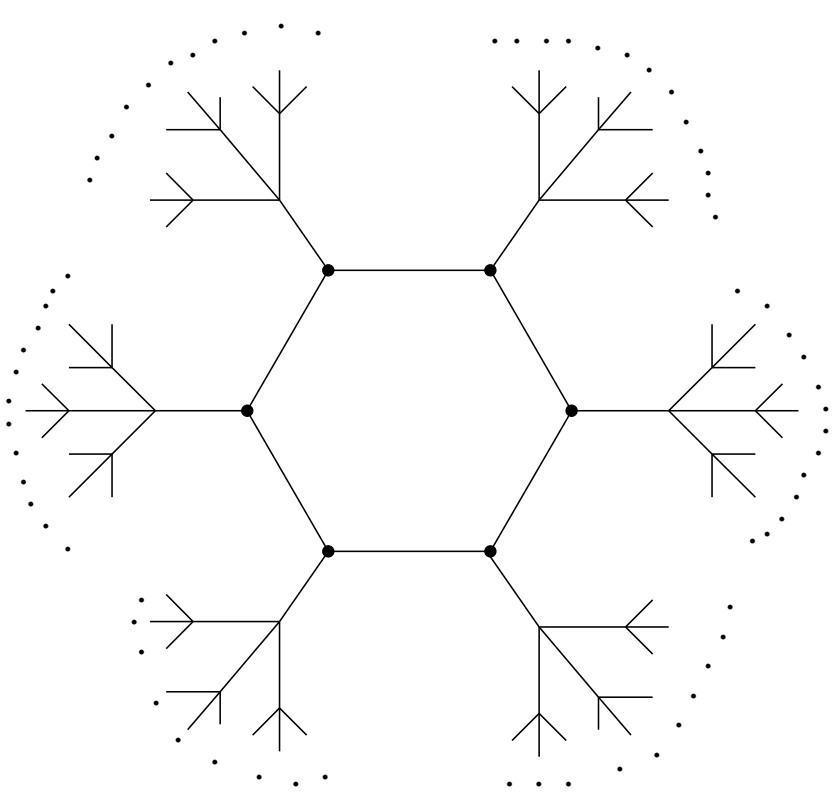

Figure 12. Mumford curve of genus one: Jacobi-Tate uniformized elliptic curve

$\Omega_{\Gamma}=\Omega_{\Gamma}(K)$ the complement $\Omega_{\Gamma}=\mathbb{P}^{1}(K) \backslash \Lambda_{\Gamma}$, that is, the domain of discontinuity of $\Gamma$.

In the case of genus $g \geq 2$ the quotient

$$
X_{\Gamma}:=\Omega_{\Gamma} / \Gamma
$$

is a Schottky-Mumford curve, with $p$-adic Schottky uniformization. The case of genus one gives the Jacobi-Tate uniformization of the elliptic curve (Figure 12).

A path in $\Delta_{K}$, infinite in both directions and with no back-tracking, is called an axis of $\Delta_{K}$. Any two points $z_{1}, z_{2} \in \mathbb{P}^{1}(K)$ uniquely define their connecting axis with endpoints at $z_{1}$ and $z_{2}$ in $\partial \Delta_{K}$. The unique axis of $\Delta_{K}$ whose ends are the fixed points of a hyperbolic element $\gamma$ is called the axis of $\gamma$. The element $\gamma$ acts on its axis as a translation. We denote by $\Delta_{\Gamma}^{\prime} \subset \Delta_{K}$ the smallest subtree containing the axes of all elements of $\Gamma$.

This subtree is $\Gamma$-invariant, with set of ends $\Lambda_{\Gamma}$. The quotient $\Delta_{\Gamma}^{\prime} / \Gamma$ is a finite graph, which is the dual graph of the closed fiber of the minimal smooth model over $\mathcal{O}$ ( $k$-split degenerate semi-stable curve) of $X_{\Gamma}$. Figure 13 shows the special fiber and the corresponding dual graph for all the possible cases of maximal degenerations special for genus two. Figure 14 shows the corresponding trees $\Delta_{\Gamma}^{\prime}$. 

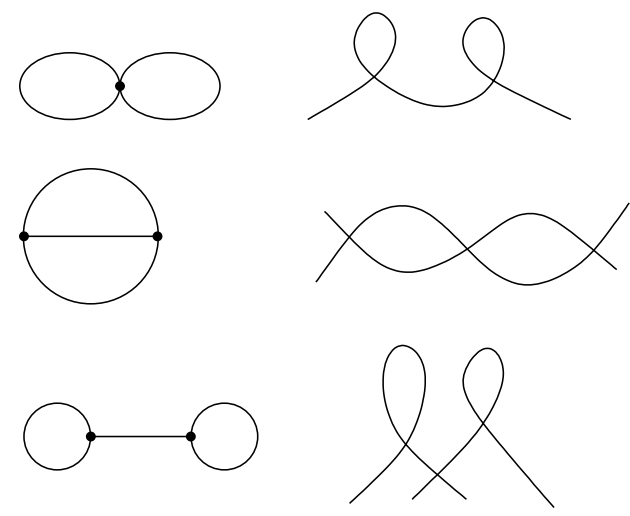

Figure 13. Genus two: special fibers and dual graphs
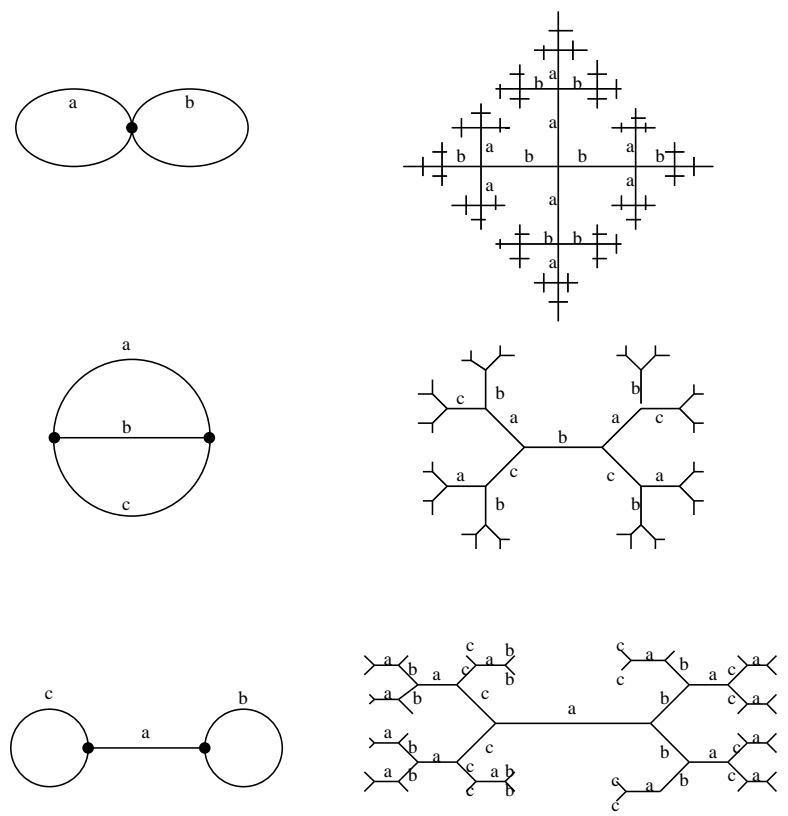

Figure 14. Genus two: trees and dual graphs

For each $n \geq 0$, we can also consider a subgraph $\Delta_{K, n}$ of the BruhatTits tree $\Delta_{K}$ defined by setting

$$
\Delta_{K, n}^{0}:=\left\{v \in \Delta_{K}^{0}: d\left(v, \Delta_{\Gamma}^{\prime}\right) \leq n\right\},
$$

where $d$ is the distance on $\Delta_{K}$ and $d\left(v, \Delta_{\Gamma}^{\prime}\right):=\inf \left\{d(v, \tilde{v}): \tilde{v} \in\left(\Delta_{\Gamma}^{\prime}\right)^{0}\right\}$, and

$$
\Delta_{K, n}^{1}:=\left\{w \in \Delta_{K}^{1}: s(w), r(w) \in \Delta_{K, n}^{0}\right\}
$$


In particular, we have $\Delta_{K, 0}=\Delta_{\Gamma}^{\prime}$.

For all $n \in \mathbb{N}$, the graph $\Delta_{K, n}$ is invariant under the action of the Schottky group $\Gamma$ on $\Delta$, and the finite graph $\Delta_{K, n} / \Gamma$ gives the dual graph of the reduction $X_{K} \otimes \mathcal{O} / \mathfrak{m}^{n+1}$.

For a more detailed account of Schottky-Mumford curves see [93] and $[80]$.

6.2. Model of the dual graph. The dictionary between the case of Mumford curves and the case at arithmetic infinity is then summarized as follows:

\begin{tabular}{|c|c|}
\hline Bruhat-Tits $\Delta_{K}$ & Hyperbolic 3 -space $\mathbb{H}^{3}$ \\
\hline $\mathbb{P}^{1}(K)=\partial \Delta_{K}$ & $\mathbb{P}^{1}(\mathbb{C})=\partial \mathbb{H}^{3}$ \\
\hline Schottky $\Gamma \subset \mathrm{PGL}(2, K)$ & Schottky $\Gamma \subset \operatorname{PSL}(2, \mathbb{C})$ \\
\hline paths in $\Delta_{K}$ & geodesics in $\mathbb{H}^{3}$ \\
\hline Mumford curve $\Omega_{\Gamma} / \Gamma$ & Riemann surface $\Omega_{\Gamma} / \Gamma$ \\
\hline tree $\Delta_{\Gamma}^{\prime}$ & convex core in $\mathbb{H}^{3}$ \\
\hline graph $\Delta_{K} / \Gamma$ & handlebody $\mathfrak{X}_{\Gamma}$ \\
\hline dual graph $\Delta_{\Gamma}^{\prime} / \Gamma$ & bounded geodesics in $\mathfrak{X}_{\Gamma}$ \\
\hline
\end{tabular}

Since we can identify bounded geodesics in $\mathfrak{X}_{\Gamma}$ with infinite geodesics in $\mathbb{H}^{3}$ with endpoints on $\Lambda_{\Gamma} \subset \mathbb{P}^{1}(\mathbb{C})$, modulo the action of $\Gamma$, these are parameterized by the complement of the diagonal in $\Lambda_{\Gamma} \times_{\Gamma} \Lambda_{\Gamma}$. This quotient is identified with the quotient of the totally disconnected space $\mathcal{S}$ of (4.4) by the action of the invertible shift $T$ of (4.5). Thus we obtain the following model for the dual graph of the fiber at infinity:

- The solenoid $\mathcal{S}_{T}$ of (4.6) is a geometric model of the dual graph of the fiber at infinity of an arithmetic surface.

- The noncommutative space $\mathcal{O}_{A}$, representing the algebra of coordinates on the quotient $\Lambda_{\Gamma} / \Gamma$, corresponds to the set of "vertices of the dual graph" (set of components of the fiber at infinity), while the noncommutative space (4.7), corresponding to the quotient $\Lambda_{\Gamma} \times_{\Gamma} \Lambda_{\Gamma}$, gives the set of "edges of the dual graph".

Moreover, using noncommutative geometry, one can also give a notion of "reduction mod $\infty$ " analogous to the reduction maps mod $p^{m}$ defined by the graphs $\Delta_{K, n}$ in the case of Mumford curves.

In fact, the reduction map corresponds to the paths connecting ends of the graph $\Delta_{K, n} / \Gamma$ to corresponding vertices of the graph $\Delta_{\Gamma}^{\prime} / \Gamma=$ $\Delta_{K, 0} / \Gamma$. The analog at arithmetic infinity consists then of geodesics in $\mathfrak{X}_{\Gamma}$ which are the images of geodesics in $\mathbb{H}^{3}$ starting at some point $x_{0} \in \mathbb{H}^{3} \cup \Omega_{\Gamma}$ and having the other end at a point of $\Lambda_{\Gamma}$. These are 
parameterized by the set

$$
\Lambda_{\Gamma} \times_{\Gamma}\left(\mathbb{H}^{3} \cup \Omega_{\Gamma}\right) .
$$

Thus, in terms of noncommutative geometry, the reduction $\bmod \infty$ corresponds to a compactification of the homotopy quotient

$$
\Lambda_{\Gamma} \times_{\Gamma} \mathbb{H}^{3}=\Lambda_{\Gamma} \times_{\Gamma} \underline{E} \Gamma
$$

where

$$
\underline{B} \Gamma=\mathbb{H}^{3} / \Gamma=\mathfrak{X}_{\Gamma} .
$$

Thus, we can view $\Lambda_{\Gamma} / \Gamma$ as the quotient of a foliation on the homotopy quotient with contractible leaves $\mathbb{H}^{3}$. The reduction $\bmod \infty$ is then given by the $\mu$-map

$$
\mu: K^{*+1}\left(\Lambda_{\Gamma} \times_{\Gamma} \mathbb{H}^{3}\right) \rightarrow K_{*}\left(C\left(\Lambda_{\Gamma}\right) \rtimes \Gamma\right) .
$$

This shows that the noncommutative space $\left(\mathcal{O}_{A}, \mathcal{H}, \mathcal{D}\right)$ is closely related ot the geometry of the fiber at arithmetic infinity of an algebraic variety. One can ask then what arithmetic information is captured by the Dirac operator $\mathcal{D}$ of this spectral triples. We'll see in the next section that (as proved in [44]), the Dirac operator gives another important arithmetic invariant, namely the local $L$-factor at the archimedean prime.

\section{Arithmetic varieties and $L$-factors}

An important invariant of arithmetic varieties is the $L$-function. This is written as a product of contributions from the finite primes and the archimedean primes,

$$
\prod_{\wp \in \operatorname{Spec} O_{\mathbb{K}}} L_{\wp}\left(H^{m}(X), s\right) .
$$

We do not plan to give a detailed account on the subject, but we refer the interested reader to [103]. Here we only try to convey some basic ideas.

The reason why one needs to consider also the contribution of the archimedean primes can be seen in the case of the "affine line" $\operatorname{Spec}(\mathbb{Z})$, where one has the Riemann zeta function, which is written as the Euler product

$$
\zeta(s)=\prod_{p}\left(1-p^{-s}\right)^{-1}
$$

However, to have a nice functional equation, one needs to consider the product

$$
\zeta(s) \Gamma(s / 2) \pi^{-s / 2},
$$


which includes a contribution of the archimedean prime, expressed in terms of the Gamma function

$$
\Gamma(s)=\int_{0}^{\infty} e^{-t} t^{s-1} d t .
$$

An analogy with ordinary geometry suggests to think of the functional equation as a sort of "Poincaré duality", which holds for a compact manifold, hence the need to "compactify" arithmetic varieties by adding the archimedean primes and the corresponding archimedean fibers.

When one looks at an arithmetic variety over a finite prime $\wp \in$ $\operatorname{Spec}\left(O_{\mathbb{K}}\right)$, the fact that the reduction lives over a residue field of positive characteristic implies that there is a special operator, the geometric Frobenius $\mathrm{Fr}_{\wp}^{*}$, acting on a suitable cohomology theory (étale cohomology), induced by the Frobenius automorphism $\phi_{\wp}$ of $\operatorname{Gal}\left(\overline{\mathbb{F}}_{p} / \mathbb{F}_{p}\right)$.

The local $L$-factors of (4.31) at finite primes encode the action of the geometric Frobenius in the form [103]

$$
L_{\wp}\left(H^{m}(X), s\right)=\operatorname{det}\left(1-F r_{\wp}^{*} N(\wp)^{-s} \mid H^{m}\left(\bar{X}, \mathbb{Q}_{\ell}\right)^{I_{\wp}}\right)^{-1} .
$$

Here we are considering the action of the geometric Frobenius $\mathrm{Fr}_{\wp}^{*}$ on the inertia invariants $H^{m}\left(\bar{X}, \mathbb{Q}_{\ell}\right)^{I_{\wp}}$ of the étale cohomology. An introduction to étale cohomology and a precise definition of these arithmetic structures is beyond the scope of these notes. In fact, our primary concern will only be the contribution of the archimedean primes to (4.31), where the construction will be based on ordinary de Rham cohomology. Thus, we only give a quick and somewhat heuristic explanation of (4.35). We refer to [103] [107] for a detailed and rigorous account and for the precise hypotheses under which the following holds.

For $X$ a smooth projective algebraic variety (in any dimension) defined over $\mathbb{Q}$, the notation $\bar{X}$ denotes

$$
\bar{X}:=X \otimes \operatorname{Spec}(\overline{\mathbb{Q}}),
$$

where $\overline{\mathbb{Q}}$ is an algebraic closure. For $\ell$ a prime, the cohomology $H^{*}\left(\bar{X}, \mathbb{Q}_{\ell}\right)$ is a finite dimensional $\mathbb{Q}_{\ell}$-vector space satisfying

$$
H^{i}(X(\mathbb{C}), \mathbb{C}) \simeq H^{i}\left(\bar{X}, \mathbb{Q}_{\ell}\right) \otimes \mathbb{C} .
$$

The absolute Galois group $\operatorname{Gal}(\overline{\mathbb{Q}} / \mathbb{Q})$ acts on $H^{*}\left(\bar{X}, \mathbb{Q}_{\ell}\right)$.

Similarly, we can consider $H^{*}\left(\bar{X}, \mathbb{Q}_{\ell}\right)$ for $X$ defined over a number field $\mathbb{K}$. For $\wp \in \operatorname{Spec}\left(O_{\mathbb{K}}\right)$ and $\ell$ a prime such that $(\ell, q)=1$, where $q$ is the cardinality of the residue field at $\wp$, the inertia invariants

$$
H^{*}\left(\bar{X}, \mathbb{Q}_{\ell}\right)^{I_{\wp}} \subset H^{*}\left(\bar{X}, \mathbb{Q}_{\ell}\right)
$$


are the part of the $\ell$-adic cohomology where the inertia group at $\wp$ acts trivially. The latter is defined as

$$
I_{\wp}=\operatorname{Ker}\left(D_{\wp} \rightarrow \operatorname{Gal}\left(\overline{\mathbb{F}}_{p} / \mathbb{F}_{p}\right)\right),
$$

with $D_{\wp}=\{\sigma \in \operatorname{Gal}(\overline{\mathbb{Q}} / \mathbb{Q}) \mid \sigma(\wp)=\wp\}$. The Frobenius automorphism of $\operatorname{Gal}\left(\overline{\mathbb{F}}_{p} / \mathbb{F}_{p}\right)$ lifts to $\phi_{\wp} \in D_{\wp} / I_{\wp}$ and induces the geometric Frobenius $F_{\wp}^{*}:=\left(\phi_{\wp}^{-1}\right)^{*}$ acting on $H^{*}\left(\bar{X}, \mathbb{Q}_{\ell}\right)^{I_{\wp}}$. We use the notation $N$ in (4.35) for the norm map. Thus, we can write the local $L$-factor (4.35) equivalently as

$$
L_{\wp}\left(H^{m}(X), s\right)=\prod_{\lambda \in \operatorname{Spec}\left(F r_{\wp}^{*}\right)}\left(1-\lambda q^{-s}\right)^{-\operatorname{dim} H^{m}\left(X_{\Gamma}\right)_{\lambda}^{I_{\wp}}},
$$

where $H^{m}\left(X_{\Gamma}\right)_{\lambda}^{I_{\wp}}$ is the eigenspace of the Frobenius with eigenvalue $\lambda$.

For our purposes, what is most important to retain from the discussion above is that the local $L$-factor (4.35) depends upon the data

$$
\left(H^{*}\left(\bar{X}, \mathbb{Q}_{\ell}\right)^{I_{\wp}}, F r_{\wp}^{*}\right)
$$

of a vector space, which has a cohomological interpretation, together with a linear operator.

7.1. Archimedean $L$-factors. Since étale cohomology satisfies the compatibility (4.36), if we again resort to the general philosophy, according to which we can work with the smooth complex manifold $X(\mathbb{C})$ and gain information on the "closed fiber" at arithmetic infinity, we are led to expect that the contribution of the archimedean primes to the $L$-function may be expressed in terms of the cohomology $H^{*}(X(\mathbb{C}), \mathbb{C})$, or equivalently in terms of de Rham cohomology.

In fact, Serre showed $([\mathbf{1 0 3}])$ that the expected contribution of the archimedean primes depends upon the Hodge structure

$$
H^{m}(X(\mathbb{C}))=\oplus_{p+q=m} H^{p, q}(X(\mathbb{C}))
$$

and is again expressed in terms of Gamma functions, as in the case of (4.33). Namely, one has a product of Gamma functions according to the Hodge numbers $h^{p, q}$,

$$
L\left(H^{*}, s\right)=\left\{\begin{array}{l}
\prod_{p, q} \Gamma_{\mathbb{C}}(s-\min (p, q))^{h^{p, q}} \\
\prod_{p<q} \Gamma_{\mathbb{C}}(s-p)^{h^{p, q}} \prod_{p} \Gamma_{\mathbb{R}}(s-p)^{h^{p,+}} \Gamma_{\mathbb{R}}(s-p+1)^{h^{p,-}}
\end{array}\right.
$$

where the two cases correspond, respectively, to the complex and the real embeddings. Here $h^{p, \pm}$ is the dimension of the $\pm(-1)^{p}$-eigenspace of the involution on $H^{p, p}$ induced by the real structure and

$$
\Gamma_{\mathbb{C}}(s):=(2 \pi)^{-s} \Gamma(s), \quad \Gamma_{\mathbb{R}}(s):=2^{-1 / 2} \pi^{-s / 2} \Gamma(s / 2) .
$$


One of the general ideas in arithmetic geometry is that one should always seek a unified picture of what happens at the finite and at the infinite primes. In particular, there should be a suitable reformulation of the local factors (4.35) and (4.42) where both formulae can be expressed in the same way.

Seeking a unified description of local $L$-factors at finite and infinite primes, Deninger in [52], [53], [54] expressed both (4.35) and (4.42) as infinite determinants.

Recall that the Ray-Singer determinant of an operator $T$ with pure point spectrum with finite multiplicities $\left\{m_{\lambda}\right\}_{\lambda \in \operatorname{Spec}(T)}$ is defined as

$$
\operatorname{det}_{\infty}(s-T):=\exp \left(-\frac{d}{d z} \zeta_{T}(s, z)_{\mid z=0}\right)
$$

where the zeta function of $T$ is defined as

$$
\zeta_{T}(s, z)=\sum_{\lambda \in \operatorname{Spec}(T)} m_{\lambda}(s-\lambda)^{-z} .
$$

Suitable conditions for the convergence of these expressions in the case of the local factors are described in [78].

Deninger showed that (4.39) can be written equivalently in the form

$$
L_{\wp}\left(H^{m}(X), s\right)^{-1}=\operatorname{det}_{\infty}\left(s-\Theta_{q}\right),
$$

for an operator with spectrum

$\operatorname{Spec}\left(s-\Theta_{q}\right)=\left\{\frac{2 \pi i}{\log q}\left(\frac{\log q}{2 \pi i}\left(s-\alpha_{\lambda}\right)+n\right): n \in \mathbb{Z}, \lambda \in \operatorname{Spec}\left(F r_{v}^{*}\right)\right\}$,

with multiplicities $d_{\lambda}$ and with $q^{\alpha_{\lambda}}=\lambda$.

Moreover, the local factor (4.42) at infinity can be written similarly in the form

$$
L\left(H^{q}(X), s\right)=\operatorname{det}_{\infty}\left(\left.\frac{1}{2 \pi}(s-\Phi)\right|_{\mathcal{H}^{m}}\right)^{-1}
$$

where $\mathcal{H}^{m}$ is an infinite dimensional vector space and $\Phi$ is a linear operator with spectrum $\operatorname{Spec}(\Phi)=\mathbb{Z}$ and finite multiplicites. This operator is regarded as a "logarithm of Frobenius" at arithmetic infinity.

Given Deninger's formulae (4.46) and (4.48), it is natural to ask for a cohomological interpretation of the data

$$
\left(\mathcal{H}^{m}, \Phi\right)
$$

somewhat analogous to (4.40). 
7.2. Arithmetic surfaces: $L$-factor and Dirac operator. Let us now return to the special case of arithmetic surfaces, in the case of genus $g \geq 2$.

At an archimedean prime, we consider the Riemann surface $X_{\alpha}(\mathbb{C})$ with a Schottky uniformization

$$
X(\mathbb{C})=\Omega_{\Gamma} / \Gamma .
$$

In the case of a real embedding we can assume that the choice of Schottky uniformization is the one that corresponds to the real structure, obtained by cutting $X(\mathbb{C})$ along the real locus $X(\mathbb{R})$.

Consider the spectral triple $\left(\mathcal{O}_{A}, \mathcal{H}, \mathcal{D}\right)$ associated to the Schottky group $\Gamma$ acting on its limit set $\Lambda_{\Gamma}$. Since the spectral triple is not finitely summable, we do not have zeta functions of the spectral triple in the form $\operatorname{Tr}\left(a|\mathcal{D}|^{z}\right)$. However, we can consider the restriction of $\mathcal{D}$ to a suitable subspace of $\mathcal{H}$, where it becomes of trace class.

In particular, consider the zeta function

$$
\zeta_{\pi_{\mathcal{V}, \mathcal{D}}}(s, z):=\sum_{\lambda \in \operatorname{Spec}(\mathcal{D})} \operatorname{Tr}\left(\pi_{\mathcal{V}} \Pi(\lambda, \mathcal{D})\right)(s-\lambda)^{-z}
$$

where $\pi_{\mathcal{V}}$ is the orthogonal projection of $\mathcal{H}$ onto the subspace $\mathcal{V}$ of $0 \oplus \mathcal{L} \subset \mathcal{H}$ defined by

$$
\Pi_{n} \pi_{\mathcal{V}} \Pi_{n}=\sum_{i=1}^{g} S_{i}^{n} S_{i}^{* n}
$$

on $\mathcal{L}$, where $\Pi_{n}$ are the spectral projections of the Dirac operator, $\Pi_{n}=\Pi(-n, \mathcal{D})$, for $n \geq 0$.

In the case of an arithmetic surface the interesting local factor is the one for the first cohomology, $L\left(H^{1}(X), s\right)$. This can be computed ([44], [46]) from the zeta function (4.50) of the spectral triple $\left(\mathcal{O}_{A}, \mathcal{H}, \mathcal{D}\right)$.

TheOREM 7.1. The local L-factor (4.42) is given by

$$
L\left(H^{1}(X), s\right)=\exp \left(-\left.\frac{d}{d z} \zeta_{\pi \mathcal{V}, \frac{\mathcal{D}}{2 \pi}}\left(\frac{s}{2 \pi}, z\right)\right|_{z=0}\right)^{-1}
$$

In the case of a real embedding, the same holds, with the projection $\pi_{\mathcal{V}, \bar{F}_{\infty}=i d}$ onto the +1 eigenspace of the involution $\bar{F}_{\infty}$ induced by the real structure on $\mathcal{V}$.

This result shows in particular that, for the special case of arithmetic surfaces with $X(\mathbb{C})$ of genus $g \geq 2$, the pair

$$
\left(\mathcal{V} \subset \mathcal{L},\left.\mathcal{D}\right|_{\mathcal{V}}\right)
$$


is a possible geometric construction of the pair $\left(\mathcal{H}^{1}, \Phi\right)$ of $(4.49)$. In particular, the Dirac operator of the spectral triple has an arithmetic meaning, in as it recovers the "logarithm of Frobenius"

$$
\left.\mathcal{D}\right|_{\mathcal{V}}=\Phi
$$

If we look more closely at the subspace $\mathcal{V}$ of the Hilbert space $\mathcal{H}$, we see that it has a simple geometric interpretation in terms of the geodesics in the handlebody $\mathfrak{X}_{\Gamma}$. Recall that the filtered subspace $\mathcal{P}$ of $\mathcal{L}=L^{2}\left(\Lambda_{\Gamma}, d \mu\right)$ describes 1 -cochains on the mapping torus $\mathcal{S}_{T}$, with $\mathcal{P} / \delta \mathcal{P}=H^{1}\left(\mathcal{S}_{T}\right)$.

The mapping torus $\mathcal{S}_{T}$ is a copy of the tangle of bounded geodesics inside $\mathfrak{X}_{\Gamma}$. Among these geodesics there are $g$ "fundamental" closed geodesics that correspond to the generators of $\Gamma$ (they correspond to geodesics in $\mathbb{H}^{3}$ connecting the fixed points $\left\{z^{ \pm}\left(\gamma_{i}\right)\right\}$, for $\left.i=1, \ldots, g\right)$. Topologically, these are the $g$ core handles of the handlebody $\mathfrak{X}_{\Gamma}$ and they generate the homology $H_{1}\left(\mathfrak{X}_{\Gamma}\right)$.

Consider the cohomology $H^{1}\left(\mathcal{S}_{T}\right)$. Suppose that we wish to find elements of $H^{1}\left(\mathcal{S}_{T}\right)$ supported on these fundamental closed geodesics. This is impossible, because forms on $\mathcal{S}_{T}$ are defined by functions in $C(\mathcal{S}, \mathbb{Z})$, that are supported on some clopen set covering the totally disconnected set $\mathcal{S}$, which contains no isolated points. However, it is possible to choose a sequence of 1-cochains on $\mathcal{S}_{T}$ whose supports are smaller and smaller clopen sets containing the infinite word in $\mathcal{S}$ corresponding to one of the $g$ fundamental geodesics. The finite dimensional subspace $\mathcal{V}_{n}=\mathcal{V} \cap \mathcal{P}_{n}$

$$
\mathcal{V}_{n} \subset \mathcal{P}_{n} \quad \operatorname{dim} \mathcal{V}_{n}=2 g
$$

gives representatives of exactly such cohomology classes in $H^{1}\left(\mathcal{S}_{T}\right)$. (We get $2 g$ instead of $g$ because we take into account the two possible choices of orientation.)

Thus, this gives us, in the case of arithmetic surfaces, a cohomological interpretation of the space $\mathcal{H}^{1}=\mathcal{V}$ in the pair (4.49). Moreover, the Schottky uniformization also provides us with a way of expressing this cohomology $\mathcal{H}^{1}=\mathcal{V}$ in terms of the de Rham cohomology $H^{1}(X(\mathbb{C})$ ). In fact, we have already seen in the calculation of the Green function that, under the hypothesis that $\operatorname{dim}_{H}\left(\Lambda_{\Gamma}\right)<1$, to each generator $\gamma_{i}$ of the Schottky group we can associate a holomorphic differential on the Riemann surface $X(\mathbb{C})$ by $(4.24)$. The map

$$
\gamma_{i} \mapsto \omega_{\gamma_{i}}=\sum_{h \in C\left(\mid \gamma_{i}\right)} d \log \left\langle h z^{+}\left(\gamma_{i}\right), h z^{-}\left(\gamma_{i}\right), z, z_{0}\right\rangle
$$




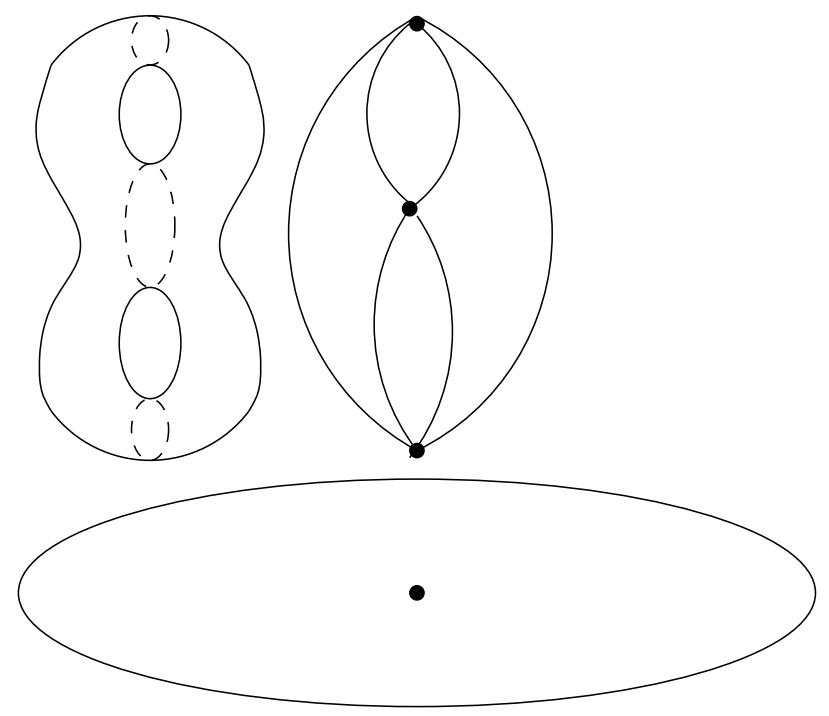

Figure 15. Vanishing cycles

thus gives an identification

$$
\mathcal{V} \simeq \oplus_{n \in \mathbb{Z}_{\geq 0}} H^{1}(X(\mathbb{C}))
$$

We'll see in the next section that, in fact, the right hand side of (4.55) is a particular case of a more general construction that works for arithmetic varieties in any dimension and that gives a cohomological interpretation of (4.49).

\section{Archimedean cohomology}

Consani gave in $[43]$, for general arithmetic varieties (in any dimension), a cohomological interpretation of the pair $\left(\mathcal{H}^{m}, \Phi\right)$ in Deninger's calculation of the archimedean $L$-factors as regularized determinants.

Her construction was motivated by the analogy between geometry at arithmetic infinity and the classical geometry of a degeneration over a disk. She introduced a double complex of differential forms with an endomorphism $N$ representing the "logarithm of the monodromy" around the special fiber at arithmetic infinity, which is modelled on (a resolution of) the complex of nearby cycles in the geometric case. The definition of the complex of nearby cycles and of its resolution, on which the following construction is modelled, is rather technical. What is easier to visualize geometrically is the related complex of the vanishing cycles of a geometric degeneration (Figure 15).

We describe here the construction of [43] using the notation of [48]. We construct the cohomology theory underlying (4.49) in several steps. 
In the following we let $X=X(\mathbb{C})$ be a complex compact Kähler manifold.

Step 1: We begin by considering a doubly infinite graded complex

$$
C^{\bullet}=\Omega^{\bullet}(X) \otimes \mathbb{C}\left[U, U^{-1}\right] \otimes \mathbb{C}\left[\hbar, \hbar^{-1}\right],
$$

where $\Omega^{\bullet}(X)$ is the de Rham complex of differential forms on $X$, while $U$ and $\hbar$ are formal variables, with $U$ of degree two and $\hbar$ of degree zero.

On this complex we consider differentials

$$
d_{C}^{\prime}:=\hbar d, \quad d_{C}^{\prime \prime}=\sqrt{-1}(\bar{\partial}-\partial),
$$

with total differential $\delta_{C}=d_{C}^{\prime}+d_{C}^{\prime \prime}$.

We also have an inner product

$$
\left\langle\alpha \otimes U^{r} \otimes \hbar^{k}, \eta \otimes U^{s} \otimes \hbar^{t}\right\rangle:=\langle\alpha, \eta\rangle \delta_{r, s} \delta_{k, t}
$$

where $\langle\alpha, \eta\rangle$ is the usual Hodge inner product of forms,

$$
\langle\alpha, \eta\rangle=\int_{X} \alpha \wedge * C(\bar{\eta}),
$$

with $C(\eta)=(\sqrt{-1})^{p-q}$, for $\eta \in \Omega^{p, q}(X)$.

Step 2: We use the Hodge filtration

$$
F^{p} \Omega^{m}(X):=\oplus_{p^{\prime}+q=m, p^{\prime} \geq p} \Omega^{p^{\prime}, q}(X)
$$

to define linear subspaces of (4.56) of the form

$$
\mathfrak{C}^{m, 2 r}=\bigoplus_{\substack{p+q=m \\ k \geq \max \{0,2 r+m\}}} F^{m+r-k} \Omega^{m}(X) \otimes U^{r} \otimes \hbar^{k}
$$

and the $\mathbb{Z}$-graded vector space

$$
\mathfrak{C}^{\bullet}=\oplus \bullet=m+2 r \mathfrak{C}^{m, 2 r} .
$$

Step 3: We pass to a real vector space by considering

$$
\mathcal{T}^{\bullet}=\left(\mathfrak{C}^{\bullet}\right)^{c=i d},
$$

where $c$ denotes complex conjugation.

In terms of the intersection of the Hodge filtrations

$$
\gamma^{\bullet}=F^{\bullet} \cap \bar{F}^{\bullet}
$$

we can write (4.63) as

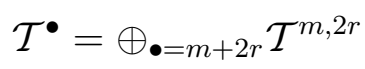


where

$$
\mathcal{T}^{m, 2 r}=\bigoplus_{\substack{p+q=m \\ k \geq \max \{0,2 r+m\}}} \gamma^{m+r-k} \Omega^{m}(X) \otimes U^{r} \otimes \hbar^{k} .
$$

The $\mathbb{Z}$-graded complex vector space $\mathfrak{C}^{\bullet}$ is a subcomplex of $C^{\bullet}$ with respect to the differential $d_{C}^{\prime}$, and for $P^{\perp}$ the orthogonal projection onto $\mathfrak{C}^{\bullet}$ in the inner product (4.58), we obtain a second differential $d^{\prime \prime}=P^{\perp} d_{C}^{\prime \prime}$. Similarly, $d^{\prime}=d_{C}^{\prime}$ and $d^{\prime \prime}=P^{\perp} d_{C}^{\prime \prime}$ define differentials on the $\mathbb{Z}$-graded real vector space $\mathcal{T}^{\bullet}$, since the inner product (4.58) is real on real forms and induces an inner product on $\mathcal{T}^{\bullet}$. We write $\delta=d^{\prime}+d^{\prime \prime}$ for the total differential.

We can describe the real vector spaces $\mathcal{T}^{\bullet}$ in terms of certain cutoffs on the indices of the complex $C^{\bullet}$. Namely, for

$$
\Lambda_{p, q}=\left\{(r, k) \in \mathbb{Z}^{2}: k \geq \kappa(p, q, r)\right\}
$$

with

$$
\kappa(p, q, r):=\max \left\{0,2 r+m, \frac{|p-q|+2 r+m}{2}\right\}
$$

(cf. Figure 16), we identify $\mathcal{T}^{\bullet}$ as a real vector space with the span

$$
\mathcal{T}^{\bullet}=\mathbb{R}\left\langle\alpha \otimes U^{r} \otimes \hbar^{k}\right\rangle,
$$

where $(r, k) \in \Lambda_{p, q}$ for $\alpha=\xi+\bar{\xi}$, with $\xi \in \Omega^{p, q}(X)$.

8.1. Operators. The complex $\left(\mathcal{T}^{\bullet}, \delta\right)$ has some interesting structures given by the action of certain linear operators.

We have the operators $N$ and $\Phi$ that correspond to the "logarithm of the monodromy" and the "logarithm of Frobenius". These are of the form

$$
N=U \hbar \quad \Phi=-U \frac{\partial}{\partial U}
$$

and they satisfy $\left[N, d^{\prime}\right]=\left[N, d^{\prime \prime}\right]=0$ and $\left[\Phi, d^{\prime}\right]=\left[\Phi, d^{\prime \prime}\right]=0$, hence they induce operators in cohomology.

Moreover, there is another important operator, which corresponds to the Lefschetz operator on forms,

$$
\mathbb{L}: \eta \otimes U^{r} \otimes \hbar^{k} \mapsto \eta \wedge \omega \otimes U^{r-1} \otimes \hbar^{k},
$$

where $\omega$ is the Kähler form on the manifold $X$. This satisfies $\left[\mathbb{L}, d^{\prime}\right]=$ $\left[\mathbb{L}, d^{\prime \prime}\right]=0$, so it also descends to the cohomology.

The pairs of operators $N$ and $\Phi$ or $\mathbb{L}$ and $\Phi$ satisfy the interesting commutation relations

$$
[\Phi, N]=-N, \quad[\Phi, \mathbb{L}]=\mathbb{L}
$$




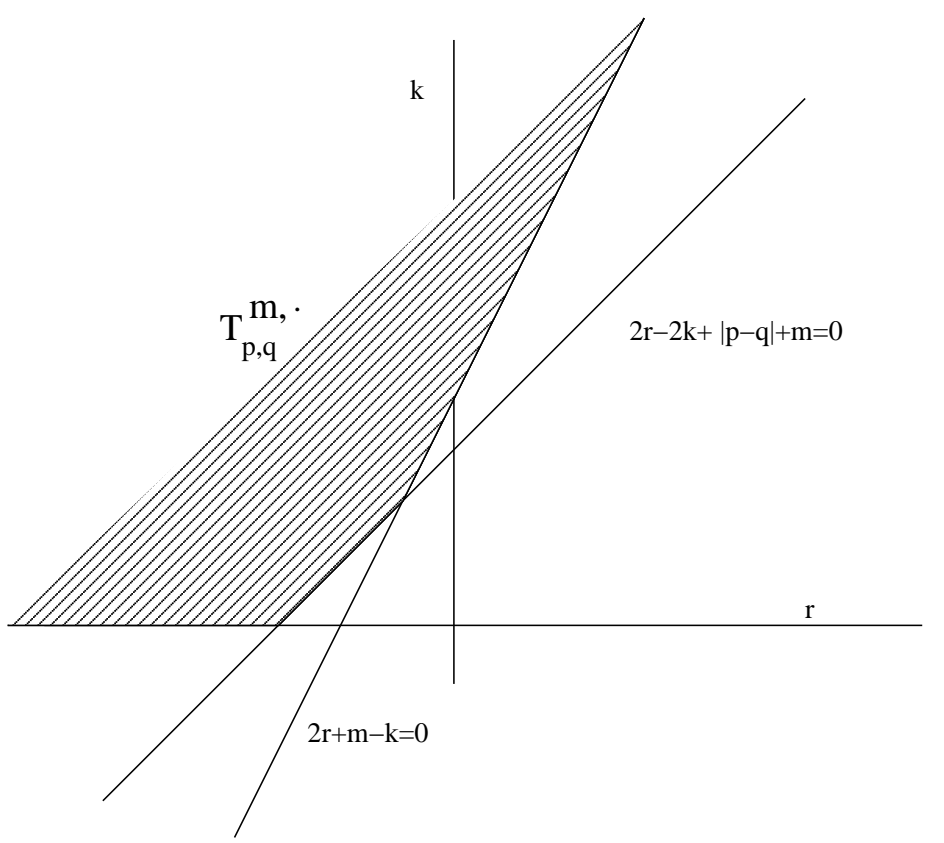

Figure 16. Cutoffs defining the complex at arithmetic infinity

that can be seen as an action of the ring of differential operators

$$
\mathbb{C}[P, Q] /(P Q-Q P=Q) .
$$

8.2. $\operatorname{SL}(2, \mathbb{R})$ Representations. Another important piece of the structure of $\left(\mathcal{T}^{\bullet}, \delta\right)$ are two involutions

$$
\begin{gathered}
S: \alpha \otimes U^{r} \otimes \hbar^{2 r+m+\ell} \mapsto \alpha \otimes U^{-(r+m)} \otimes \hbar^{\ell} \\
\tilde{S}: \alpha \otimes U^{r} \otimes \hbar^{k} \mapsto C(* \alpha) \otimes U^{r-(n-m)} \otimes \hbar^{k} .
\end{gathered}
$$

These maps, together with the nilpotent operators $N$ and $\mathbb{L}$ define two representations of $\mathrm{SL}(2, \mathbb{R})$. In terms of

$$
\begin{aligned}
& \nu(s):=\left(\begin{array}{cc}
s & 0 \\
0 & s^{-1}
\end{array}\right) s \in \mathbb{R}^{*} \\
& u(t):=\left(\begin{array}{ll}
1 & t \\
0 & 1
\end{array}\right) t \in \mathbb{R} \\
& w:=\left(\begin{array}{cc}
0 & 1 \\
-1 & 0
\end{array}\right),
\end{aligned}
$$


the representations $\sigma^{L}$ and $\sigma^{R}$ are given by

$$
\begin{array}{lrl}
\sigma^{L}(\nu(s))=s^{-n+m} & \sigma^{R}(\nu(s))=s^{2 r+m} \\
\sigma^{L}(u(t))=\exp (t \mathbb{L}) & \sigma^{R}(u(t))=\exp (t N) \\
\sigma^{L}(w)=(\sqrt{-1})^{n} C \tilde{S} & \sigma^{R}(w)=C S .
\end{array}
$$

Of these representations, $\sigma^{L}$ extends to an action by bounded operators on the Hilbert completion of $\mathcal{T}^{\bullet}$ in the inner product (4.58), while the action of the subgroup $\nu(s), s \in \mathbb{R}^{*}$ of $\operatorname{SL}(2, \mathbb{R})$ via the representation $\sigma^{R}$ on this Hilbert space is by unbounded densely defined operators.

8.3. Renormalization group and monodromy. This very general structure, which exists for varieties in any dimension, also has interesting connections to noncommutative geometry. For instance, we can see that in fact the map $N$ does play the role of the "logarithm of the monodromy" using an analog in our context of the theory of renormalization à la Connes-Kreimer [31].

In the classical case of a geometric degeneration on a disk, the monodromy around the special fiber is defined as the map

$$
T=\exp \left(-2 \pi \sqrt{-1} \operatorname{Res}_{0}(\nabla)\right)
$$

where

$$
N=\operatorname{Res}_{0}(\nabla)
$$

is the residue at zero of the connection, acting as an endomorphism of the cohomology.

In our setting, we consider loops $\phi_{\mu}$ with values in the group $G=$ $\operatorname{Aut}\left(\mathcal{T}_{\mathbb{C}}^{\bullet}, \delta\right)$, depending on a "mass parameter" $\mu \in \mathbb{C}^{*}$. Here $\mathcal{T}_{\mathbb{C}}$ is the complexification of the real vector space $\mathcal{T}^{\bullet}$. The Birkhoff decomposition of a loop $\phi_{\mu}$ consists in the multiplicative decomposition

$$
\phi_{\mu}(z)=\phi_{\mu}^{-}(z)^{-1} \phi_{\mu}^{+}(z)
$$

for $z \in \partial \Delta \subset \mathbb{P}^{1}(\mathbb{C})$, where $\Delta$ is a small disk centered at zero. Of the two terms in the right hand side of $(4.77), \phi_{\mu}^{+}$extends to a holomorphic function on $\Delta$ and $\phi_{\mu}^{-}$to a holomorphic functions on $\mathbb{P}^{1}(\mathbb{C}) \backslash \Delta$ with values in $G$. We normalize $(4.77)$ by requiring that $\phi_{\mu}^{-}(\infty)=1$.

By analogy with the Connes-Kreimer theory of renormalization we require the following properties of (4.77):

- The time evolution

$$
\theta_{t}: a \mapsto e^{-t \Phi} a e^{t \Phi}
$$


acts by scaling

$$
\phi_{\lambda \mu}(\epsilon)=\theta_{t \epsilon} \phi_{\mu}(\epsilon)
$$

for $\lambda=e^{t} \in \mathbb{R}_{+}^{*}$ and $\epsilon \in \partial \Delta$.

- The term $\phi_{\mu}^{-}=\phi^{-}$in the Birkhoff decomposition is independent of the energy scale $\mu$.

The residue of a loop $\phi_{\mu}$, as in $[\mathbf{3 1}]$, is given by

$$
\operatorname{Res} \phi=\left.\frac{d}{d z}\left(\phi^{-}(1 / z)^{-1}\right)\right|_{z=0}
$$

and the beta function of renormalization is

$$
\beta=\Upsilon \operatorname{Res} \phi, \quad \text { with } \quad \Upsilon=\left.\frac{d}{d t} \theta_{t}\right|_{t=0} .
$$

In our setting, there is a natural choice of the time evolution, given by the "geodesic flow" associated to the "Dirac operator" $\Phi$, namely

$$
\theta_{t}(a)=e^{-t \Phi} a e^{t \Phi}
$$

This gives

$$
\Upsilon(a)=\left.\frac{d}{d t} \theta_{t}(a)\right|_{t=0}=[a, \Phi] .
$$

There is a scattering formula $([\mathbf{3 1}])$, by which one can reconstruct $\phi^{-}$from the residue. Namely, we can write

$$
\phi^{-}(z)^{-1}=1+\sum_{k \geq 1} d_{k} z^{-k}
$$

with

$$
d_{k}=\int_{s_{1} \geq \cdots \geq s_{k} \geq 0} \theta_{-s_{1}}(\beta) \cdots \theta_{-s_{k}}(\beta) d s_{1} \cdots d s_{k} .
$$

The renormalization group is given by

$$
\rho(\lambda)=\lim _{\epsilon \rightarrow 0} \phi^{-}(\epsilon) \theta_{t \epsilon}\left(\phi^{-}(\epsilon)^{-1}\right),
$$

for $\lambda=e^{t} \in \mathbb{R}_{+}^{*}$.

Thus, we only need to specify the residue in order to have the corresponding renormalization theory associated to $\left(\mathcal{T}_{\mathbb{C}}^{\bullet}, \delta\right)$. By analogy with the case of the geometric degeneration (4.76) it is natural to require that $\operatorname{Res} \phi=N$. We then have $([48])$ :

Proposition 8.1. A loop $\phi_{\mu}$ in $G=\operatorname{Aut}\left(\mathcal{T}_{\mathbb{C}}^{\bullet}, \delta\right)$ with $\operatorname{Res} \phi_{\mu}=N$, subject to (4.79) and with $\phi^{-}$independent of $\mu$, satisfies

$$
\phi_{\mu}(z)=\exp \left(\frac{\mu^{z}}{z} N\right)
$$


with Birkhoff decomposition

$$
\phi_{\mu}(z)=\exp (-N / z) \exp \left(\frac{\mu^{z}-1}{z} N\right) .
$$

In fact, by (4.83) and $\operatorname{Res} \phi_{\mu}=N$ we have $\beta=[N, \Phi]=N$ and $\theta_{t}(N)=e^{t} N$, hence the scattering formula (4.84) gives

$$
\phi^{-}(z)=\exp (-N / z)
$$

and the scaling property (4.79) determines

$$
\phi_{\mu}(z)=\exp \left(\frac{\mu^{z}}{z} N\right) \text {. }
$$

The part of the Birkhoff decomposition that is regular at $z=0$ satisfies $\phi_{\mu}^{+}(0)=\mu^{N}$.

The renormalization group is of the form

$$
\rho(\lambda)=\lambda^{N}=\exp (t N),
$$

which, through the representation $\sigma^{R}$ of $\mathrm{SL}(2, \mathbb{R})$, corresponds to the horocycle flow on $\operatorname{SL}(2, \mathbb{R})$

$$
\rho(\lambda)=u(t)=\left(\begin{array}{ll}
1 & t \\
0 & 1
\end{array}\right) .
$$

The Birkhoff decomposition (4.77) gives a trivialization of a principal $G$-bundle over $\mathbb{P}^{1}(\mathbb{C})$. We can consider the associated vector bundle $\mathcal{E}_{\mu}^{\bullet}$ with fiber $\mathcal{T}_{\mathbb{C}}^{\bullet}$

Moreover, we obtain a Fuchsian connection $\nabla_{\mu}$ on this bundle,

$$
\nabla_{\mu}: \mathcal{E}_{\mu}^{\bullet} \rightarrow \mathcal{E}_{\mu}^{\bullet} \otimes_{\mathcal{O}_{\Delta}} \Omega_{\Delta}^{\bullet}(\log 0),
$$

where the notation $\Omega_{\Delta}^{\bullet}(\log 0)$ denotes forms with logarithmic poles at 0 . This has the form

$$
\nabla_{\mu}=N\left(\frac{1}{z}+\frac{d}{d z} \frac{\mu^{z}-1}{z}\right) d z
$$

with local gauge potentials

$$
-\phi^{+}(z)^{-1} \frac{\log \pi(\gamma) d z}{z} \phi^{+}(z)+\phi^{+}(z)^{-1} d \phi^{+}(z)
$$

with respect to the monodromy representation

$$
\pi: \pi_{1}\left(\Delta^{*}\right)=\mathbb{Z} \rightarrow G
$$

given by

$$
\pi(\gamma)=\exp (-2 \pi \sqrt{-1} N)
$$


for $\gamma$ the generator of $\pi_{1}\left(\Delta^{*}\right)$. This corresponds to (4.75) in the classical geometric case. This Fuchsian connection has residue

$$
\operatorname{Res}_{z=0} \nabla_{\mu}=N
$$

as in the geometric case (4.76).

8.4. Local factor and archimedean cohomology. In [43] Consani showed that the data $\left(\mathcal{H}^{m}, \Phi\right)$ of $(4.49)$ can be identified with

$$
\left(\mathbb{H}^{\bullet}\left(\mathcal{T}^{\bullet}, \delta\right)^{N=0}, \Phi\right),
$$

where $\mathbb{H}^{\bullet}\left(\mathcal{T}^{\bullet}, \delta\right)$ is the hypercohomology (the cohomology with respect to the total differential $\delta$ ) of the complex $\mathcal{T}^{\bullet}$ and $\mathbb{H}^{\bullet}\left(\mathcal{T}^{\bullet}, \delta\right)^{N=0}$ is the kernel of the map induced by $N$ on cohomology. The operator $\Phi$ is the one induced on cohomology by that of (4.69). She called $\mathcal{H}^{m} \simeq$ $\mathbb{H}^{\bullet}\left(\mathcal{T}^{\bullet}, \delta\right)^{N=0}$ the archimedean cohomology.

This can also be viewed $(c f .[\mathbf{4 3}])$ as a piece of the cohomology of the cone of the monodromy $N$. This is the complex

$$
\operatorname{Cone}(N)^{\bullet}=\mathcal{T}^{\bullet} \oplus \mathcal{T}^{\bullet}[+1]
$$

with differential

$$
D=\left(\begin{array}{cc}
\delta & -N \\
0 & \delta
\end{array}\right) .
$$

The complex (4.89) inherits a positive definite inner product from $\mathcal{T}^{\bullet}$, which descends on cohomology. The representation $\sigma^{L}$ of $\operatorname{SL}(2, \mathbb{R})$ on $\mathcal{T}^{\bullet}$ induces a representation on Cone $(N)^{\bullet}$. The corresponding representation $d \sigma^{L}: \mathfrak{g} \rightarrow \operatorname{End}\left(\mathcal{T}^{\bullet}\right)$ of the Lie algebra $\mathfrak{g}=\operatorname{sl}(2, \mathbb{R})$ extends to a representation of the universal enveloping algebra $U(\mathfrak{g})$ on $\mathcal{T} \bullet$ and on Cone $(N)^{\bullet}$. This gives a representation in the algebra of bounded operators on the Hilbert completion of $\operatorname{Cone}(N)^{\bullet}$ in the inner product.

THEOREM 8.2. The triple

$$
(\mathcal{A}, \mathcal{H}, \mathcal{D})=\left(U(\mathfrak{g}), \mathbb{H}^{\bullet}(\text { Cone }(N)), \Phi\right)
$$

has the properties that $\mathcal{D}=\mathcal{D}^{*}$ and that $\left(1+\mathcal{D}^{2}\right)^{-1 / 2}$ is a compact operator. The commutators $[\mathcal{D}, a]$ are bounded operators for all $a \in$ $U(\mathfrak{g})$ and the triple is $1^{+}$-summable.

Thus, $(\mathcal{A}, \mathcal{H}, \mathcal{D})$ has most of the properties of a spectral triple, confirming the fact that the logarithm of Frobenius $\Phi$ should be thought of as a Dirac operator.

In any case, the structure is sufficient to consider zeta functions for this "spectral triple". In particular, we can recover the alternating products of the local $L$-factors at infinity from a zeta function of the spectral triple. 
TheOREM 8.3. Consider the zeta function

$$
\zeta_{a, \Phi}(z)=\operatorname{Tr}\left(a|\Phi|^{-z}\right)
$$

with $a=\sigma^{L}(w)$. This gives

$$
\operatorname{det}_{\infty \sigma^{L}(w), \Phi}(s)=\prod_{m=0}^{2 n} L\left(H^{m}(X), s\right)^{(-1)^{m}} .
$$

8.5. Arithmetic surfaces: homology and cohomology. In the particular case of arithmetic surfaces, there is an identification $([43],[44])$

$$
\mathbb{H}^{\bullet}(\operatorname{Cone}(N)) \simeq \mathcal{H}^{\bullet} \oplus \check{\mathcal{H}} \bullet
$$

where $\mathcal{H}^{\bullet}$ is the archimedean cohomology and $\check{\mathcal{H}}^{\bullet}$ is its dual under the involution $S$ of (4.71).

We can then extend the identification

$$
U: \mathcal{H}^{1} \stackrel{\simeq}{\rightarrow} \mathcal{V} \subset \mathcal{L}
$$

of (4.55), by considering a subspace $\mathcal{W}$ of the homology $H_{1}\left(\mathcal{S}_{T}\right)$ with $\mathcal{W} \simeq \check{\mathcal{H}}^{\bullet}$. The homology $H_{1}\left(\mathcal{S}_{T}\right)$ can also be computed as a direct limit

$$
H_{1}\left(\mathcal{S}_{T}, \mathbb{Z}\right)=\underset{N}{\lim } \mathcal{K}_{N}
$$

where the $\mathcal{K}_{N}$ are free abelian of rank $(2 g-1)^{N}+1$ for $N$ even and $(2 g-1)^{N}+(2 g-1)$ for $N$ odd. The $\mathbb{Z}$-module $\mathcal{K}_{N}$ is generated by the closed geodesics represented by periodic sequences in $\mathcal{S}$ of period $N+1$. These need not be primitive closed geodesics. In terms of primitive closed geodesics we can write equivalently

$$
H_{1}\left(\mathcal{S}_{T}, \mathbb{Z}\right)=\oplus_{N=0}^{\infty} \mathcal{R}_{N}
$$

where the $\mathcal{R}_{N}$ are free abelian groups with

$$
\operatorname{rk} \mathcal{R}_{N}=\frac{1}{N} \sum_{d \mid N} \mu(d) \mathrm{rk} \mathcal{K}_{N / d}
$$

with $\mu(d)$ the Möbius function satisfying $\sum_{d \mid N} \mu(d)=\delta_{N, 1}$.

The pairing of homology and cohomology is given by

$$
\langle\cdot, \cdot\rangle: F_{n} \times \mathcal{K}_{N} \rightarrow \mathbb{Z} \quad\langle[f], x\rangle=N \cdot f(\bar{x}) .
$$


This determines a graded subspace $\mathcal{W} \subset H_{1}\left(\mathcal{S}_{T}, \mathbb{Z}\right)$ dual to $\mathcal{V} \subset$ $H^{1}\left(\mathcal{S}_{T}\right)$. With the identification

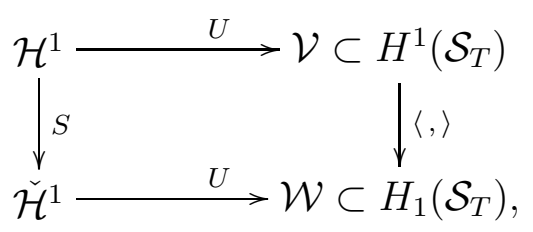

we can identify the Dirac operator of (4.16) with the logarithm of Frobenius

$$
\left.\mathcal{D}\right|_{\mathcal{V} \oplus \mathcal{V}}=\Phi_{\mathcal{H}^{1} \oplus \check{\mathcal{H}}^{1}}
$$




\section{CHAPTER 5}

\section{Vistas}

This last brief chapter is meant to answer the question "where do we go from here?", namely, to outline just a few among the many possible directions in which the field is evolving. Some of the topics listed below are recent results, some are more speculative thoughts. It all just serves the purpose of conveying the sense of a certain momentum acquired by this set of topics. More detailed information on recent results in "arithmetic noncommutative geometry" will be found in the volume $[49]$ and in the monograph in preparation $[37]$.

Noncommutative geometry of Shimura varieties. The results of $[\mathbf{9}],[32]$ and $[38]$ can be seen as arising from a noncommutative version of the Shimura varieties $S h\left(\mathrm{GL}_{1}, \pm 1\right)$ and $S h\left(\mathrm{GL}_{2}, \mathbb{H}^{ \pm}\right)$. The results of [83] on the noncommutative boundary of the modular curves and the modular Hecke algebras of $[\mathbf{4 1}][\mathbf{4 2}]$ also contribute to illustrate the rich noncommutative geometry associated to the modular tower, i.e. to the Shimura variety $S h\left(\mathrm{GL}_{2}, \mathbb{H}^{ \pm}\right)$.

It is then a natural idea to seek for a larger class of quantum statistical mechanical systems associated to more general Shimura varieties, and investigate the arithmetic properties of low temperature KMS states. Shimura varieties have the typical property that they have adelic groups of symmetries, so that the problem of compatibility between adelic symmetries and the Galois action on the values of zero temperature KMS states on an arithmetic subalgebra can be formulated very naturally in this context.

A program aimed at constructing analogs of the Bost-Connes system and of the $\mathrm{GL}_{2}$ system of [32] for a large class of Shimura varieties is being pursued by Eugene Ha and Frédéric Paugam. In their construction, one considers a more general Shimura datum $(G, X)$. The analog of passing from $\mathrm{GL}_{2}\left(\mathbb{A}_{f}\right)$ to $M_{2}\left(A_{f}\right)$ in the $\mathrm{GL}_{2}$ system, or from $\mathbb{A}_{f}^{*}$ to $\mathbb{A}_{f}$ in the Bost-Connes case, is here achieved by considering a suitable enveloping semigroup $M$. To the resulting datum $(G, X, M)$ they associate a groupoid (in the category of stacks) whose convolution algebra generalizes the algebra $\mathcal{A}_{2}$ of the $\mathrm{GL}_{2}$-system. Their algebra comes with variants depending on the choice of a level structure (which 
can be used to avoid the stacky singularities in the groupoid). There is a natural time evolution on the resulting algebra, which is defined in terms of the data of the level structure, and the resulting system has a rich adèlic group of symmetries. In particular these results can be seen very explicitly in the Hilbert modular case. They also obtain through this general method quantum statistical mechanical systems naturally associated to number fields. Their construction agrees with that of [38] for imaginary quadratic fields. The ongoing investigation of the arithmetic properties of KMS states for these systems will certainly yield interesting new results.

Classical Shimura varieties also admit noncommutative boundaries, in the same sense in which a noncommutative boundary of modular curves was constructed in [83]. F. Paugam studied such noncommutative boundaries [96] [97], described by (the convolution algebras associated to) double cosets spaces $L \backslash G(\mathbb{R}) / P(K)$ where $G$ is a connected reductive algebraic group over $\mathbb{Q}, L$ is an arithmetic subgroup of $G(\mathbb{Q})$, and $P(K)$ is a real parabolic subgroup in $G(\mathbb{R})$. As moduli spaces they describe degenerations of complex structures on tori in multifoliations. Paugam's point of view proceeds largely form Hodge theoretic considerations.

A question, in this general perspective, that seems very interesting is whether one can establish a connection between this type of results on the noncommutative geometry of modular curves and Darmon's generalization of the theory of Heegner points [27].

Spectral triples and arithmetic geometry. Besides the occurrence of spectral triples in the context of the archimedean factors at arithmetic infinity, discussed in the last part of this book, it would be interesting to seek other occurrences in number theoretic context.

For example, there may be natural spectral triples associated to the algebras of the quantum statistical mechanical systems for noncommutative Shimura varieties mentioned above.

In the case of the Bost-Connes system, this should be interestingly related to the noncommutative space of Connes' spectral realization of the zeros of the Riemann zeta function, and for the $\mathrm{GL}_{2}$-system to the modular Hecke algebras of Connes-Moscovici hence to modular forms. The cases of more general Shimura varieties mentioned above should also be interesting to consider in this perspective.

Another class of spaces of arithmetic significance that may carry interesting spectral triples are spaces that, like the Mumford curves (cf. $[\mathbf{4 7}]$ ), admit $p$-adic uniformizations (for instance in the case of the Cherednik-Drinfeld theory of $p$-adic uniformization of Shimura curves), 
as well as generalizations of Bruhat-Tits trees with the action of p-adic Schottky groups, given by certain classes of higher rank buildings. Constructions of this type may also be interestingly related to Berkovich's spaces, or to Buium's arithmetic differential invariants.

Hopf algebra actions. While symmetries of ordinary commutative spaces are encoded by group actions, symmetries of noncommutative spaces are typically given by Hopf algebra actions.

In the context of relations between noncommutative geometry and number theory, this important structure of noncommutative spaces was exploited in the work of Connes and Moscovici on the modular Hecke algebras $[\mathbf{4 1}][\mathbf{4 2}]$, where it proves to be a very powerful technique. The modular Hecke algebras combine the product of modular forms with the action of Hecke operators and can be seen as the "holomorphic part" of the algebra of coordinates of the noncommutative space of commensurability classes of 2-dimensional $\mathbb{Q}$-lattices discussed above in Chapter 3. They are endowed with symmetries given by the action of the Connes-Moscovici Hopf algebra $\mathcal{H}_{1}$ of transverse geometry in codimension one. This Hopf algebra arises in the context of foliations and it acts naturally on crossed products. It is part of a family of Hopf algebras $\mathcal{H}_{n}$ of transverse geometry in codimension $n$, obtained from the dual of the enveloping algebra of the Lie algebra of vector fields [40].

The action of this Hopf algebra on the modular Hecke algebras is determined by a grading operator, corresponding to the weight of modular forms, a derivation introduced by Ramanujan, which corrects the ordinary differentiation by a logarithmic derivative of the Dedekind $\eta$ function, and an operator that acts as multiplication by a form-valued cocycle on $\mathrm{GL}_{2}^{+}(\mathbb{Q})$ that measures the lack of modular invariance of $\eta^{4} d z$. This allow one to transfer notions and results from the transverse geometry of foliations to the context of modular forms and Hecke operators.

A tool, which we did not discuss in this book, which is very useful in dealing with Hopf algebra symmetries of noncommutative spaces, is the Hopf cyclic cohomology of Hopf algebras, introduced by Connes and Moscovici and more recently refined through a systematic foundational treatment by Khalkhali and Rangipour ( $c f$. [64] for an overview). It can be thought of as the right analog of group and Lie algebra cohomology in noncommutative geometry.

In the case of the modular Hecke algebras, the use of Hopf cyclic cohomology yields interesting results about modular forms. In the case of the Hopf algebra $\mathcal{H}_{1}$, there are three basic cyclic cocycles, which in 
the original context of transverse geometry correspond, respectively, to the Schwarzian derivative, the Godbillon-Vey class, and the transverse fundamental class. The cocycle associated to the Schwarzian derivative is realized by an inner derivation, given in terms of the Eisenstein series

$$
E_{4}(q)=1+240 \sum_{1}^{\infty} n^{3} \frac{q^{n}}{1-q^{n}} .
$$

This cyclic cocycle has an important arithmetic significance, related to the data used by Zagier in defining "canonical" Rankin-Cohen algebras in [110]. The cocycle associated to the Godbillon-Vey class is expressed in terms of a 1-cocycle on $\mathrm{GL}_{2}^{+}(\mathbb{Q})$ with values in Eisenstein series of weight two. The cocycle associated to the transverse fundamental class gives rise to a natural extension of the first Rankin-Cohen brackets of modular forms (cf. [110]).

Renormalization and motivic Galois symmetry. We have seen in the last chapter an application of the formalism of the ConnesKreimer theory of perturbative renormalization in the context of arithmetic geometry. In fact, a closer inspection of the Connes-Kreimer formalism itself revealed surprising connections to arithmetic, in the original context of perturbative renormalization of quantum field theories. This was investigated in joint work of Alain Connes and the author $[34],[35],[36]$.

There, we started from the formulation of perturbative renormalization in terms of Birkhoff decomposition of loops, in the form recalled here in (4.77) above, with values in the pro-unipotent Lie group $G(\mathbb{C})$ of complex points of the affine group scheme associated to the ConnesKreimer Hopf algebra of Feynman graphs of [30]. We showed that the scattering formula of Connes-Kreimer, which appeared in this text in (4.84), can be conveniently formulated in terms of iterated integrals and the time ordered exponential, or expansional. This follows from a calculation generalizing that of Proposition 8.1 above, which gives the time ordered exponential expression

$$
\phi^{-}(z)=T e^{-\frac{1}{z} \int_{0}^{\infty} \theta_{-t}(\beta) d t} .
$$

Once expressed in expansional form as above, one can understand the terms in the Birkhoff decomposition as solutions of certain differential equations. This leads to a reformulation of perturbative renormalization in terms of the equivalence classes of certain differential systems with singularities.

The nature of the singularities is determined by the two conditions on the behavior of the terms in the Birkhoff decomposition with respect 
to the scaling of the mass parameter, namely the conditions $\partial_{\mu} \phi_{\mu}^{-}=0$ and $\phi_{e^{t} \mu}(\epsilon)=\theta_{t \epsilon} \phi_{\mu}(\epsilon)$, mentioned in the last chapter.

These two conditions are expressed geometrically through the notion of $G$-valued equisingular connections on a principal $\mathbb{C}^{*}$-bundle $B$ over a disk $\Delta$, where $G$ is the pro-unipotent Lie group of characters of the Connes-Kreimer Hopf algebra of Feynman graphs. The equisingularity condition is the property that such a connection $\omega$ is $\mathbb{C}^{*}$-invariant and that its restrictions to sections of the principal bundle that agree at $0 \in \Delta$ are mutually equivalent, in the sense that they are related by a gauge transformation by a $G$-valued $\mathbb{C}^{*}$-invariant map regular in $B$.

Thus, the geometric formulation equivalent to the data of perturbative renormalization is given by a class (up to equivalence given by such gauge transformations) of flat equisingular $G$-valued connections. The class of differential systems defined by the flat equisingular connections can be studied with techniques of differential Galois theory. In particular, one can identify this way an underlying group of symmetries, the differential Galois group. In fact, we show that the category of equivalence classes of flat equisingular bundles is a neutral Tannakian category, which is equivalent to the category of finite dimensional linear representations of an affine group scheme $U^{*}=U \rtimes \mathbb{G}_{m}$, with $U$ a prounipotent affine group scheme and $\mathbb{G}_{m}$ the multiplicative group. The renormalization group lifts canonically to a one-parameter subgroup of $U$ and this gives it an interpretation as a group of Galois symmetries.

The affine group scheme $U$ corresponds to the free graded Lie algebra with one generator in each degree $n \in \mathbb{N}$. These corresponds to "splitting up" the renormalization group flow in homogeneous components by loop number. This affine group scheme admits an arithmetic interpretation as the motivic Galois group of the category of mixed Tate motives on the scheme of N-cyclotomic integers (after localization at $N$ ), for $N=3$ or $N=4$.

The generator of the renormalization group lifted to $U$ defines a $u n i$ versal singular frame whose explicit expression in the generators of the Lie algebra has the same rational coefficients that appear in the local index formula of Connes-Moscovici [39]. This appears as an intriguing connection between perturbative renormalization and noncommutative geometry, possibly through an interpretation of the local index formula in terms of chiral anomalies.

Noncommutative algebraic geometry. Throughout this book, we followed ideas and methods of noncommutative geometry in the form developed by Connes, which we very briefly summarized in the 
initial chapter. There exist other variants of noncommutative geometry, which are closer in spirit to algebraic geometry, for instance the versions of "noncommutative algebraic geometry" developed by Artin, Tate, and van den Bergh, or by Kontsevich and Rosenberg. For the interested reader, a nice short survey of directions and results in noncommutative algebraic geometry was written recently by S. Mahanta $[73]$.

One may wonder whether more algebraic versions of noncommutative geometry may be more suitable for application to problems of algebraic number theory and arithmetic algebraic geometry. Certainly, one can expect that a closer interplay between the various versions of noncommutative geometry, besides being desirable in terms of the internal development of the field, will help push the number theoretic applications further. The recent work of Connes and Dubois-Violette [28], [29] opens up important new perspectives that combine these two different approaches to noncommuative geometry and allow for the use of both analytic and algebro-geometric tools.

A good example of such interplay also comes from recent results of Polishchuk [98], inspired by Manin's real multiplication program, where noncommutative projective varieties are naturally associated to noncommutative tori with real multiplication. 


\section{Bibliography}

[1] N. Alling, N. Greenleaf, Foundations of the theory of Klein surfaces, Lecture Notes in Mathematics Vol. 219, Springer Verlag 1971.

[2] L. Alvarez-Gaumé, G. Moore, C. Vafa, Theta functions, modular invariance, and strings. Comm. Math. Phys. 106 (1986), no. 1, 1-40.

[3] S. Aminneborg, I. Bengtsson, D. Brill, S. Holst, P. Peldan, Black holes and wormholes in $2+1$ dimensions, Class. Quantum Grav. 15 (1998) 627-644.

[4] J. Arledge, M. Laca, I. Raeburn, Semigroup crossed products and Hecke algebras arising from number fields, Doc. Math. 2 (1997) 115-138.

[5] K. I. Babenko, On a problem of Gauss. Dokl. Akad. Nauk SSSR, Tom 238 (1978) No. 5, 1021-1024.

[6] J. D. Barrow. Chaotic behaviour and the Einstein equations. In: Classical General Relativity, eds. W. Bonnor et al., Cambridge Univ. Press, Cambridge, 1984, 25-41.

[7] P. Baum, A. Connes, Geometric K-theory for Lie groups and foliations. Preprint IHES 1982; l'Enseignement Mathematique, t. 46, 2000, 1-35.

[8] F.P. Boca, Projections in rotation algebras and theta functions, Commun. Math. Phys. 202 (1999) 325-357.

[9] J.B. Bost, A. Connes, Hecke algebras, Type III factors and phase transitions with spontaneous symmetry breaking in number theory, Selecta Math. (New Series) Vol.1 (1995) N.3, 411-457.

[10] R. Bowen, Hausdorff dimension of quasi-circles, Publ.Math. IHES 50 (1979) $11-25$.

[11] M. Boyle, D. Handelman, Orbit equivalence, flow equivalence, and ordered cohomology, Israel J. Math. 95 (1996) 169-210.

[12] O. Bratteli, D.W. Robinson, Operator algebras and quantum statistical mechanics I,II, Springer Verlag, 1981.

[13] P. Chakraborty, A. Pal, Equivariant spectral triples on the quantum SU(2) group, $K$-Theory 28 (2003), no. 2, 107-126.

[14] C.H.Chang and D.Mayer, Thermodynamic formalism and Selberg's zeta function for modular groups, Regular and chaotic dynamics 15 (2000) N.3 281-312.

[15] P.B. Cohen, $A C^{*}$-dynamical system with Dedekind zeta partition function and spontaneous symmetry breaking, Journal de Théorie des Nombres de Bordeaux 11 (1999) 15-30.

[16] A. Connes, $C^{*}$ algèbres et géométrie differentielle. C.R. Acad. Sci. Paris, Ser. A-B , 290 (1980) 599-604.

[17] A. Connes, An analogue of the Thom isomorphism for crossed products of a $C^{*}$-algebra by an action of $\mathbb{R}, \mathrm{Adv}$. in Math. 39 (1981), no. 1, 31-55. 
[18] A. Connes, Non-commutative differential geometry, Publ.Math. IHES N.62 (1985) 257-360.

[19] A. Connes, Cyclic cohomology and the transverse fundamental class of a foliation. In: Geometric methods in operator algebras (Kyoto, 1983). Pitman Res. Notes in Math., 123, Longman, Harlow 1986, 52-144.

[20] A. Connes, Compact metric spaces, Fredholm modules, and hyperfiniteness, Ergod. Th. Dynam. Sys. (1989) 9, 207-220.

[21] A. Connes, Noncommutative geometry, Academic Press, 1994.

[22] A. Connes, Geometry from the spectral point of view. Lett. Math. Phys. 34 (1995), no. 3, 203-238.

[23] A. Connes, Trace formula in Noncommutative Geometry and the zeros of the Riemann zeta function. Selecta Mathematica. New Ser. 5 (1999) 29-106.

[24] A. Connes, A short survey of noncommutative geometry, J. Math. Phys. 41 (2000), no. 6, 3832-3866.

[25] A. Connes, Cyclic cohomology, Quantum group Symmetries and the Local Index Formula for $S U_{q}(2)$, J. Inst. Math. Jussieu 3 (2004), no. 1, 17-68.

[26] A. Connes, M. Douglas, A. Schwarz, Noncommutative geometry and Matrix theory: compactification on tori. J. High Energy Phys. (1998) no. 2, Paper 3,35 pp. (electronic)

[27] H. Darmon, Rational points on modular elliptic curves, CBMS Regional Conference Series in Mathematics, 101. American Mathematical Society, 2004.

[28] A. Connes, M. Dubois-Violette, Noncommutative finite-dimensional manifolds. I. spherical manifolds and related examples, Comm. Math. Phys. Vol.230 (2002) N.3, 539-579.

[29] A. Connes, M. Dubois-Violette, Moduli space and structure of noncommutative 3-spheres, Lett. Math. Phys., Vol.66 (2003) N.1-2, 91-121.

[30] A. Connes, D. Kreimer, Renormalization in quantum field theory and the Riemann-Hilbert problem. I. The Hopf algebra structure of graphs and the main theorem. Comm. Math. Phys. 210 (2000), no. 1, 249-273.

[31] A. Connes, D. Kreimer, Renormalization in quantum field theory and the Riemann-Hilbert problem. II. The $\beta$-function, diffeomorphisms and the renormalization group. Comm. Math. Phys. 216 (2001), no. 1, 215-241.

[32] A. Connes, M. Marcolli, Quantum Statistical Mechanics of $\mathbb{Q}$-lattices, (From Physics to Number Theory via Noncommutative Geometry, Part I), preprint arXiv:math.NT/0404128.

[33] A. Connes, M. Marcolli, $\mathbb{Q}$-lattices: quantum statistical mechanics and Galois theory, to appear in Journal of Geometry and Physics.

[34] A. Connes, M. Marcolli, Renormalization and motivic Galois theory, Int. Math. Res. Not. 2004, no. 76, 4073-4091.

[35] A. Connes, M. Marcolli, Renormalization, the Riemann-Hilbert correspondence, and motivic Galois theory (From Physics to Number Theory via Noncommutative Geometry. Part II), preprint arXiv hep-th/0411114.

[36] A. Connes, M. Marcolli, Quantum fields and motives, to appear in Journal of Geometry and Physics.

[37] A. Connes, M. Marcolli, Noncommutative Geometry from Quantum Physics to Motives, book in preparation. 
[38] A. Connes, M. Marcolli, N. Ramachandran KMS states and complex multiplication, arXiv math.OA/0501424

[39] A. Connes, H. Moscovici, The local index formula in noncommutative geometry. Geom. Funct. Anal. 5 (1995), no. 2, 174-243.

[40] A. Connes, H. Moscovici, Cyclic cohomology and Hopf algebras, Lett. Math. Phys. 48 (1999), no. 1, 97-108.

[41] A. Connes, H. Moscovici, Modular Hecke algebras and their Hopf symmetry, Moscow Math. Journal, Vol.4 (2004) N.1, 67-109.

[42] A. Connes, H. Moscovici, Rankin-Cohen Brackets and the Hopf Algebra of Transverse Geometry, Moscow Math. Journal, Vol.4 (2004) N.1, 111-130.

[43] C. Consani, Double complexes and Euler L-factors, Compositio Math. 111 (1998) 323-358.

[44] C. Consani, M. Marcolli, Noncommutative geometry, dynamics and $\infty$-adic Arakelov geometry, to appear in Selecta Mathematica

[45] C. Consani, M. Marcolli, Triplets spectreaux in geometrie d'Arakelov, C.R.Acad.Sci. Paris, Ser. I 335 (2002) 779-784.

[46] C. Consani, M. Marcolli, New perspectives in Arakelov geometry, to appear in proceedings of CNTA7 meeting Montreal 2002.

[47] C. Consani, M. Marcolli, Spectral triples from Mumford curves, International Mathematics Research Notices 36 (2003) 1945-1972.

[48] C. Consani, M. Marcolli, Archimedean cohomology revisited, arXiv math.AG/0407480.

[49] C. Consani, M. Marcolli (editors), Proccedings of the conference "Noncommutative geometry and number theory" held in Bonn 2003. To appear.

[50] G. Cornell and J.H. Silverman (eds.) Arithmetic geometry, Springer-Verlag, New York, 1986

[51] J. Cuntz, W. Krieger, A class of $C^{*}$-algebras and topological Markov chains, Invent. Math. 56 (1980) 251-268.

[52] C. Deninger, On the $\Gamma$-factors attached to motives, Invent. Math. 104 (1991) 245-261.

[53] C. Deninger, Local L-factors of motives and regularized determinants. Invent. Math. 107 (1992), no. 1, 135-150.

[54] C. Deninger, Motivic L-functions and regularized determinants, in "Motives", Proceedings of Symposia in Pure Mathematics, Vol. 55 (1994) Part I, 707-743.

[55] V. Drinfeld, Two theorems on modular curves, Funct. Anal. and its Applications, Vol.7 (1973) N.2, 155-156.

[56] V. Drinfeld, Yu.I. Manin, Periods of p-adic Schottky groups. J. Reine Angew. Math. 262/263 (1973), 239-247.

[57] E. Ha, F. Paugam, private communication.

[58] R. Haag, Local Quantum Physics, Springer, Berlin 1992.

[59] R. Haag, N. M. Hugenholtz, M. Winnink On the equilibrium states in quantum statistical mechanics, Comm. Math. Phys. 5 (1967), 215, 236.

[60] D. Harari, E. Leichtnam, Extension du phénomène de brisure spontanée de symétrie de Bost-Connes au cas des corps globaux quelconques, Selecta Math. (New Series) Vol.3 (1997) 205-243. 
[61] G. Harder, General aspects in the theory of modular symbols, Seminar on number theory, Paris 1981-82 (Paris, 1981/1982), 73-88, Progr. Math., 38, Birkhäuser Boston, Boston, MA, 1983.

[62] D. Hensley, Continued fraction Cantor sets, Hausdorff dimension, and functional analysis, J. Number Theory 40 (1992) 336-358.

[63] I. M. Khalatnikov, E. M. Lifshitz, K. M. Khanin, L. N. Schur, Ya. G. Sinai. On the stochasticity in relativistic cosmology. J. Stat. Phys., 38:1/2 (1985), 97-114.

[64] M. Khalkhali, B. Rangipour, Introduction to Hopf-Cyclic Cohomology, preprint, arXiv math.QA/0503244.

[65] K. Krasnov, Holography and Riemann Surfaces, Adv. Theor. Math. Phys. 4 (2000) 929-979

[66] K. Krasnov, Analytic Continuation for Asymptotically AdS 3D Gravity, grqc/0111049

[67] M. Laca, Semigroups of *-endomorphisms, Dirichlet series, and phase transitions. J. Funct. Anal. 152 (1998), no. 2, 330-378.

[68] M. Laca, From endomorphisms to automorphisms and back: dilations and full corners, J. London Math. Soc. (2) 61 (2000) 893-904.

[69] S. Lang, Elliptic Functions, (Second Edition), Graduate Texts in Mathematics, Vol.112, Springer-Verlag 1987.

[70] G. Landi, An introduction to noncommutative spaces and their geometries, Lecture Notes in Physics, Vol. m-51, Springer Verlag 1997.

[71] S. Lang, Introduction to Arakelov Theory, Springer-Verlag, New York, 1988.

[72] R. Loudon, The Quantum Theory of Light, third edition, Oxford University Press, 2000.

[73] S. Mahanta, A Brief Survey of Non-Commutative Algebraic Geometry, preprint arXiv math.QA/0501166.

[74] L. Mandel, E. Wolf, Optical Coherence and Quantum Optics, Cambridge University Press, 1995.

[75] Yu.I. Manin, Von Zahlen und Figuren, arXiv math.AG/0201005.

[76] Yu.I. Manin, Real Multiplication and noncommutative geometry, arXiv math.AG/0202109.

[77] Yu.I. Manin, Theta functions, quantum tori, and Heisenberg groups, Lett. in Math. Phys. 56 (2001) 295-320.

[78] Yu.I. Manin, Lectures on zeta functions and motives (according to Deninger and Kurokawa). Columbia University Number Theory Seminar (New York, 1992). Astérisque No. 228 (1995), 4, 121-163.

[79] Yu.I. Manin, Three-dimensional hyperbolic geometry as $\infty$-adic Arakelov geometry, Invent. Math. 104 (1991) 223-244.

[80] Yu.I. Manin, p-adic automorphic functions. Journ. of Soviet Math., 5 (1976) 279-333.

[81] Yu.I. Manin, Parabolic points and zeta functions of modular curves, Math. USSR Izvestija, vol. 6 N. 1 (1972) 19-64. Selected Papers, World Scientific, 1996, 202-247.

[82] Yu.I. Manin, M. Marcolli, Holography principle and arithmetic of algebraic curves, Adv. Theor. Math. Phys. Vol.3 (2001) N.5, 617-650. 
[83] Yu.I. Manin, M. Marcolli, Continued fractions, modular symbols, and noncommutative geometry, Selecta Mathematica (New Series) Vol.8 N.3 (2002) 475-520.

[84] M. Marcolli, Limiting modular symbols and the Lyapunov spectrum, Journal of Number Theory, Vol.98 N.2 (2003) 348-376.

[85] M. Marcolli, Modular curves, $C^{*}$-algebras and chaotic cosmology, arXiv math-ph/0312035.

[86] K. Matsuzaki, M. Taniguchi, Hyperbolic manifolds and Kleinian groups, Oxford Univ. Press, 1998.

[87] D.H. Mayer, Relaxation properties of the mixmaster universe, Phys. Lett. A 121 (1987), no. 8-9, 390-394.

[88] D.H. Mayer, Continued fractions and related transformations, in "Ergodic theory, symbolic dynamics, and hyperbolic spaces", Oxford Univ. Press, 1991.

[89] B. Mazur, Courbes elliptiques et symboles modulaires, Séminaire Bourbaki vol.1971/72, Exposés 400-417, LNM 317, Springer Verlag 1973, pp. 277-294.

[90] L. Merel, Intersections sur les courbes modulaires, Manuscripta Math., 80 (1993) 283-289.

[91] J.S. Milne, Canonical models of Shimura curves, manuscript, 2003 (available from www.jmilne.org)

[92] D. Mumford (with M.Nori and P.Norman), Tata lectures on theta, III, Progress in Mathematics Vol. 97, Birkhäuser 1991.

[93] D. Mumford, An analytic construction of degenerating curves over complete local rings, Compositio Math. 24 (1972) 129-174.

[94] D. Mumford, C. Series, D. Wright, Indra's Pearls. The Vision of Felix Klein. Cambridge University Press, New York, 2002.

[95] W. Parry, S. Tuncel, Classification problems in ergodic theory, London Math. Soc. Lecture Notes Series 67, 1982.

[96] F. Paugam, Three examples of noncommutative boundaries of Shimura varieties, preprint arXiv math.AG/0410254.

[97] F. Paugam, Quelques bords irrationnels de varietes de Shimura, preprint arXiv math.AG/0410269.

[98] A. Polishchuk, Noncommutative two-tori with real multiplication as noncommutative projective varieties, J. Geom. Phys. 50 (2004), no. 1-4, 162-187.

[99] M. Pollicott, H. Weiss, Multifractal analysis of Lyapunov exponent for continued fraction and Manneville-Pomeau transformations and applications to Diophantine approximation, Comm. Math. Phys. 207 (1999), no. 1, 145-171.

[100] D.B. Ray, I.M. Singer, Analytic torsion for complex manifolds. Ann. of Math. (2) 98 (1973), 154-177.

[101] M.A. Rieffel, $\mathrm{C}^{*}$-algebras associated to irrational rotations, Pacific J. Math. 93 (1981) 415-429.

[102] A.L. Rosenberg, Noncommutative schemes. Compositio Math. 112 (1998) N.1, 93-125.

[103] J.P. Serre, Facteurs locaux des fonctions zêta des variétés algébriques (définitions et conjectures). Sém. Delange-Pisot-Poitou, exp. 19, 1969/70.

[104] G. Shimura, Arithmetic Theory of Automorphic Functions, Iwanami Shoten and Princeton 1971. 
[105] H.M. Stark, L-functions at $s=1$. IV. First derivatives at $s=0$, Adv. Math. 35 (1980) 197-235.

[106] P. Stevenhagen, Hilbert's 12th problem, complex multiplication and Shimura reciprocity, Advanced Studies in Pure Math. 30 (2001) "Class Field Theory - its centenary and prospect" pp. 161-176.

[107] A. Weil, Basic Number Theory, Springer 1974.

[108] R.O. Wells, Differential analysis on complex manifolds. Springer-Verlag, 1980.

[109] A. Werner, Local heights on Mumford curves. Math. Ann. 306 (1996), no. 4, 819-831.

[110] D. Zagier, Modular forms and differential operators, in K. G. Ramanathan memorial issue. Proc. Indian Acad. Sci. Math. Sci. 104 (1994), no. 1, 57-75. 